Grand Valley State University

ScholarWorks@GVSU

4-13-2016

\title{
Comparison of Load Carrying Capacity of Three and Four Lobed Polygonal Shaft and Hub Connection for Constant Grinding Diameter
}

\author{
Ravi Bhatta \\ Grand Valley State University
}

Follow this and additional works at: https://scholarworks.gvsu.edu/theses

\section{ScholarWorks Citation}

Bhatta, Ravi, "Comparison of Load Carrying Capacity of Three and Four Lobed Polygonal Shaft and Hub Connection for Constant Grinding Diameter" (2016). Masters Theses. 797.

https://scholarworks.gvsu.edu/theses/797

This Thesis is brought to you for free and open access by the Graduate Research and Creative Practice at ScholarWorks@GVSU. It has been accepted for inclusion in Masters Theses by an authorized administrator of ScholarWorks@GVSU. For more information, please contact scholarworks@gvsu.edu. 
Comparison of Load Carrying Capacity of Three and Four Lobed Polygonal Shaft and Hub Connection for Constant Grinding Diameter

\author{
Ravi Bhatta
}

A Thesis Submitted to the Graduate Faculty of

GRAND VALLEY STATE UNIVERSITY

In

Partial Fulfillment of the Requirements

For the Degree of

Masters of Science in Mechanical Engineering

Padnos College of Engineering and Computing

April 2016 


\section{Acknowledgements}

I would like to express my sincere gratitude to my advisor Prof. Dr. Wendy Reffeor for her immense support, vast knowledge, patience, and encouragement for completion of the thesis. Her persistent guidance helped me in all aspect of this research. I could not imagine having a better advisor and mentor for my study.

I would also like to thank the rest of my thesis committee, Prof. Dr. Princewill Anyalebechi and Prof. Dr. Sung-Hwan, for their insightful comments and encouragement that helped me get the subject matters correct.

My sincere thanks also goes to the Vice President of General Polygon Systems, Inc., Mr. Michael Sormanti, who provided me with valuable knowledge and information on polygonal shafts along with the sample pieces for my experimental verification.

I would also like to thank Mr. Jerry Smant, Engineering lab supervisor, who provided continuous support in fabricating the test setup and Mr. Russell Palmitier who helped me correct and improve my writing. 


\begin{abstract}
Polygonal shafts are a major competitor to spline and keyed shafts for power transmission due to features as self-centering, lack of stress concentration area, and ease of assembly and disassembly. Past studies on polygonal profiles have focused on a single profile and comparisons based on nominal sizes of three and four lobe profiles. This research explores the loading strength of the standardized three and four lobe polygonal shafts and hubs manufactured from the same stock size, subjected to pure torsional and torsional bending load from a spur gear of $20^{\circ}$ pressure angle at various fits. In absence of analytical solution, Finite element method has been used after verifying the results experimentally, theoretically, and DIN standard. From the finite element analysis, the hub was found to experience greater stress than the shaft in all cases. The clearance fit was found to be the most critical connection and interference fit to be the most suitable for larger power transmission. The P4C connection had greater stress, especially in the hub, than the P3G connection. The difference between the P4C shaft and the P3G shaft was 4.05\% in the interference fit and 60.6\% in the clearance fit, suggesting P4C clearance fit to be less favorable for larger power transmission. Owing to its small normal axial stress, the P4C clearance fit has its use in low power transmission where sliding fit is a requirement. The reason for greater stress in P4C shaft and hub connection is due to the large pressure angle at the point of contact, which leads to a smaller contact area and greater contact pressure. The contact pressure was found to be triangular shaped in clearance and transition fit and with a large crest, followed by a trough and a small crest in interference fit for torsional bending load.
\end{abstract}




\section{Table of Contents}

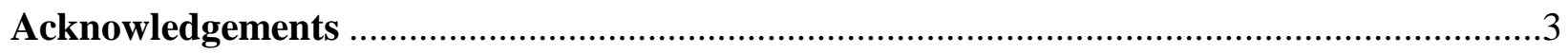

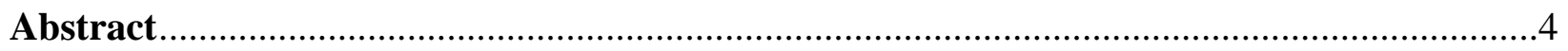

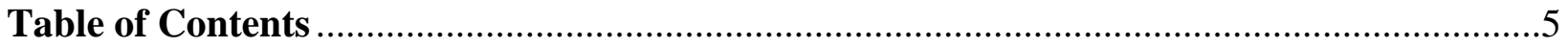

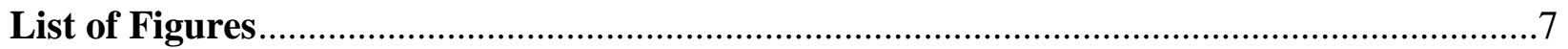

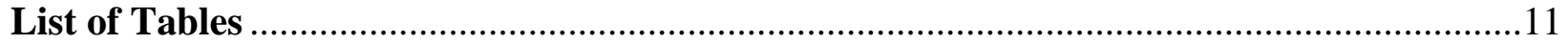

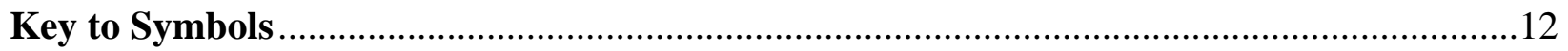

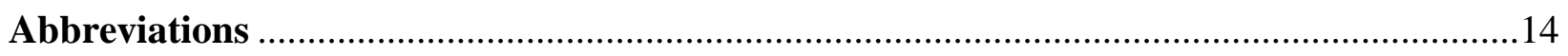

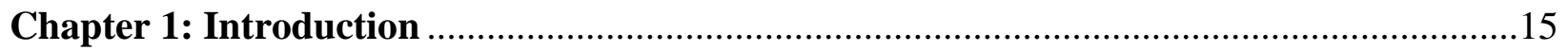

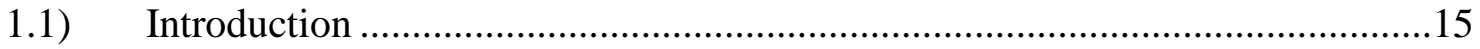

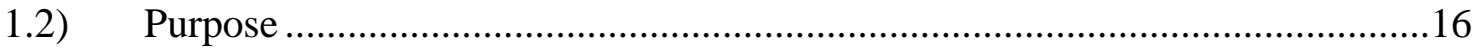

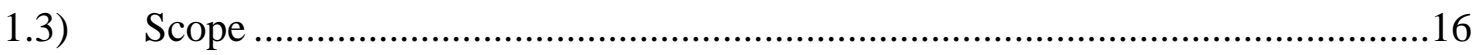

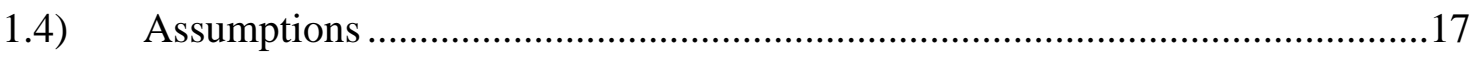

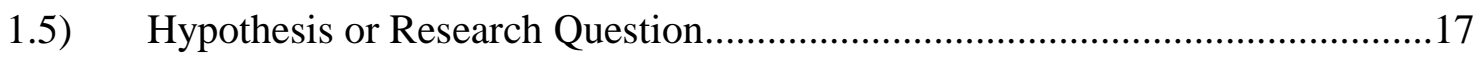

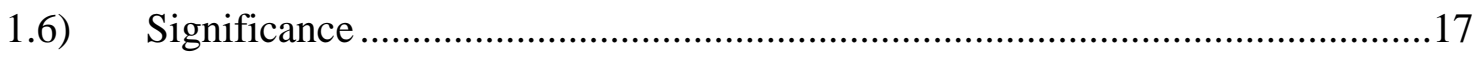

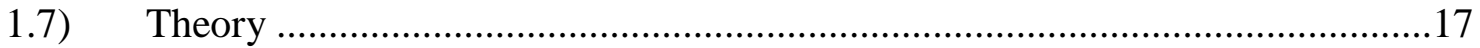

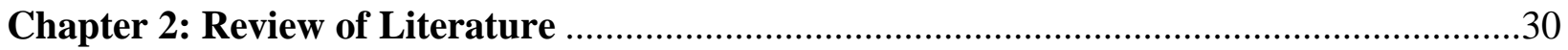

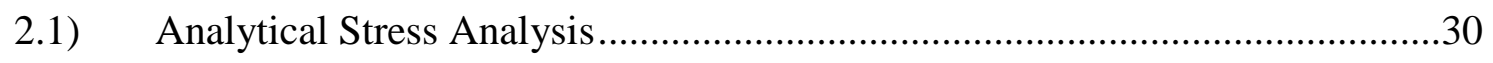

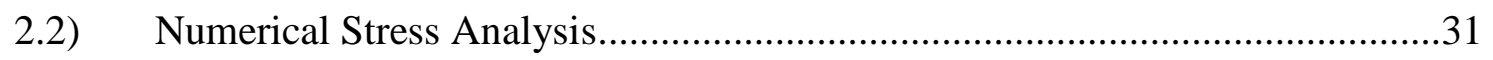

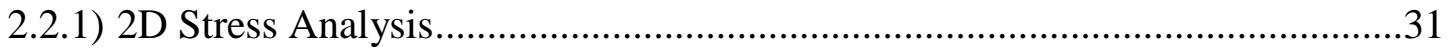

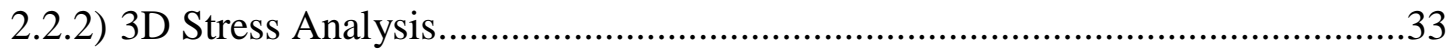

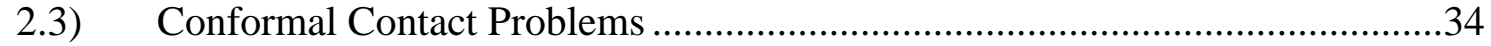

2.4) Computational Contact Mechanics ............................................................34

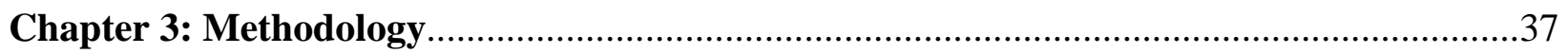

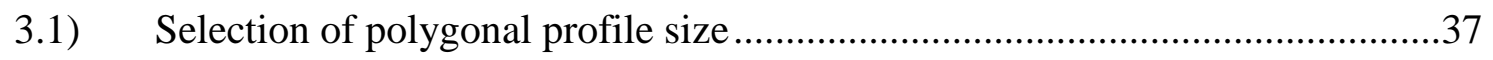

3.2) Loading in P3G and P4C profiles...........................................................39

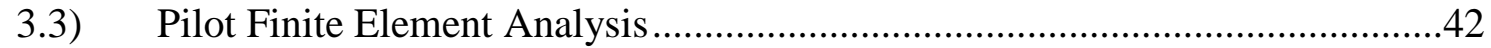

3.4) Validation of results from FEM to experimental, analytical and DIN standard48

3.5) Detailed Finite Element Analysis ...............................................................48

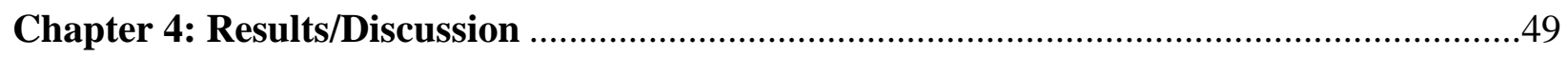

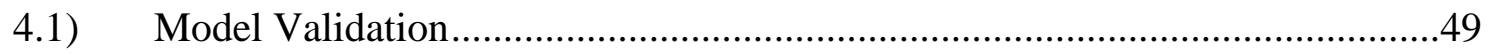




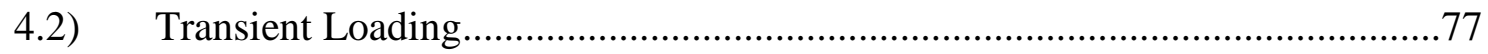

4.3) Loading capacity under torsional and torsional bending load.........................87

4.3.1) Effect of loading on Torsional Shear stress......................................................8

4.3.2) Effect of loading on Normal stress along axial direction ...............................90

4.3.3) Effect of loading on Contact Stress …..........................................................93

4.3.4) Effect of loading on Contact pressure Distribution......................................97

4.3.5) Effect of loading on maximum von Mises stress in shaft ............................103

4.3.6) Effect of loading on maximum von Mises stress in hub ..............................106

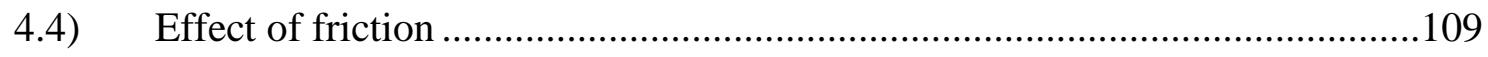

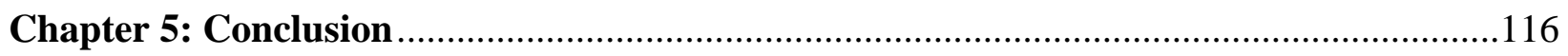

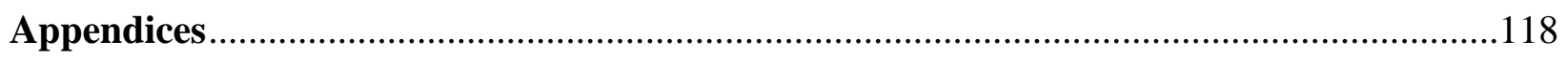

Appendix A: Dimension of the shaft and hub used in Experiment .......................119

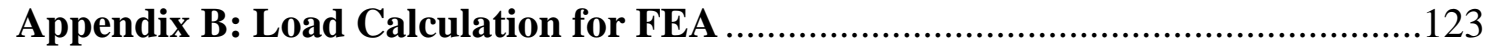

Appendix C: Experimental Stress Determination .........................................128

Appendix D: Graphical Results from FEA ................................................131

Appendix E: Permission to publish Figure 1................................................145

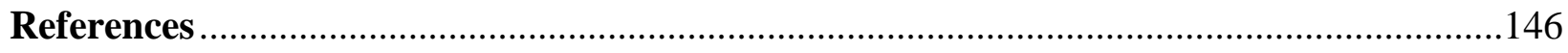




\section{List of Figures}

Figure 1: Common Epitrochoidal Curves drawn using equations (1) and (2) ........................... 18

Figure 2: Geometrical Description of a three lobe polygon curve......................................... 19

Figure 3: Different Polygonal profiles .............................................................................. 19

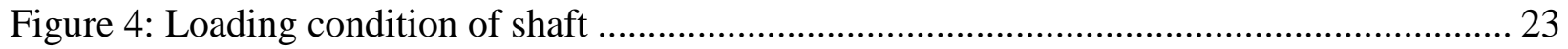

Figure 5: Forces in a P3G shaft hub connection for idealized point of contact........................... 23

Figure 6: Forces in a P4C shaft hub connection for idealized point of contact .......................... 24

Figure 7: Point of contact in P3G profile............................................................................ 26

Figure 8: Point of contact in P4C profile ..................................................................... 26

Figure 9: Effect of (a) Torsional loading and (b) Diametrical Clearance on pressure distribution

in zone of contact (Czyzewski \& Odman, 1988)................................................................. 32

Figure 10: Newton Raphson method for solving nonlinear problems .................................... 36

Figure 11: Dimension of the three lobe polygonal shaft and hub used for FEA in mm.............. 38

Figure 12: Dimension of the four lobe polygonal shaft and hub used for FEA in mm ............... 38

Figure 13: Comparison of cross section of P3G and P4C profile............................................ 39

Figure 14: Bending Load in P3G shafts for FEA ….......................................................... 40

Figure 15: Bending Load in P4C shafts for FEA................................................................. 41

Figure 16: Tetrahedron Mesh with inflation in P4C connection ............................................. 47

Figure 17: Hexahedral mesh with mid node in P4C connection ............................................... 47

Figure 18: Hexahedral mesh without mid node in P4C connection (selected mesh) .................. 48

Figure 19: Hexahedral mesh without mid node in P3G connection (selected mesh) ................. 48

Figure 20: Schematic of contact stabilization damping factor........................................... 51

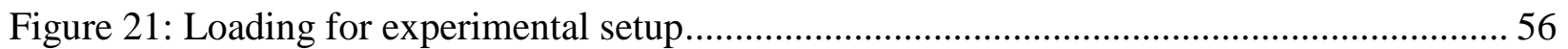

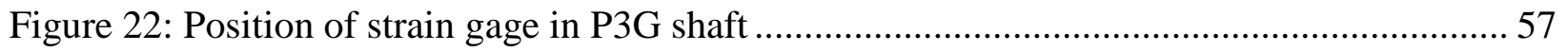

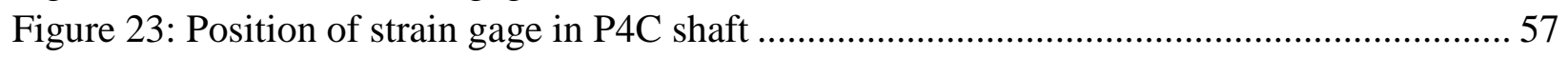

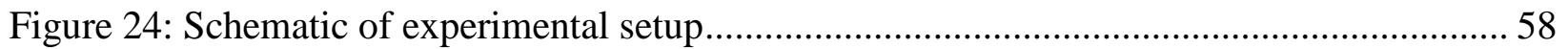

Figure 25: Experimental setup for strain measurement for P4C connection ............................. 58

Figure 26: Experimental setup for strain measurement in P3G connection ............................... 59

Figure 27: Comparison of actual (blue continuous) and approximation curve (red dotted)........ 63

Figure 28: Torque application in a three lobe polygonal shaft FEA model................................ 63

Figure 29: Comparison of FEA (blue solid) and Analytical (red dotted) result for $\tau_{\mathrm{xz}}$ of P3G shaft subjected to a torque of $80 \mathrm{Nm}$ (upper plot) with profile comparison (lower plot).................... 65 Figure 30: Comparison of FEA (blue solid) and Analytical (red dotted) result for $\tau_{\mathrm{yz}}$ of P3G shaft subjected to a torque of $80 \mathrm{Nm}$ (upper plot) with profile comparison (lower plot)..................... 66 Figure 31: Comparison of FEA (blue solid) and Analytical (red dotted) result for torsional shear stress of P3G shaft subjected to a torque of $80 \mathrm{Nm}$ (upper plot) with profile comparison (lower

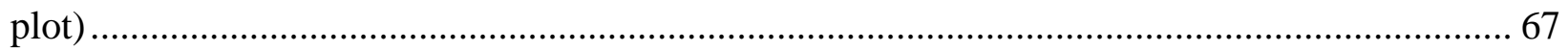

Figure 32: Comparison of actual (blue) and approximation curve (red discontinuous) .............. 69

Figure 33: Torque application in a four lobe polygonal shaft FEA model ............................... 69 
Figure 34: Comparison of FEA (blue solid) and Analytical (red dotted) result for $\tau_{\mathrm{xz}}$ of four lobe polygonal shaft subjected to a torque of $80 \mathrm{Nm}$ (upper plot) with profile comparison (lower plot)

Figure 35: Comparison of FEA (red dotted) and Analytical (blue solid) result for $\tau_{\mathrm{yz}}$ of P4C shaft subjected to a torque of $80 \mathrm{Nm}$ (upper plot) with profile comparison (lower plot)..................... 71 Figure 36: Comparison of FEA (red dotted) and Analytical (blue solid) result for resultant shear stress of four lobe polygonal shaft subjected to a torque of $80 \mathrm{Nm}$ (upper plot) with profile comparison (lower plot) .................................................................................................. 72

Figure 37: Load step (upper) and convergence (lower) for line fit P3G connection ................... 77

Figure 38: Max DOF Increment (upper) and Line search (lower) against cumulative ............... 78

Figure 39: Variation of maximum von Mises stress on the P3G hub ..................................... 79

Figure 40: Variation of maximum von Mises stress on the P3G shaft ...................................... 79

Figure 41: Variation of maximum contact stress in P3G shaft hub connection ........................ 79

Figure 42: Variation of sliding distance in P3G shaft hub contact region................................ 79

Figure 43: Variation in torsional shear stress of P3G shaft .............................................. 80

Figure 44: Load step (upper) and convergence (lower) for clearance fit P4C connection .......... 81

Figure 45: Max DOF Increment (upper) and Line search (lower) against cumulative iterations for

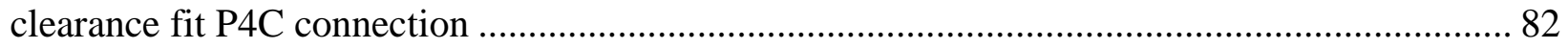

Figure 46: Variation of maximum von Mises stress on the P4C hub ........................................ 82

Figure 47: Variation of maximum von Mises stress on the P4C shaft ................................... 83

Figure 48: Variation of maximum contact stress in P4C shaft hub connection........................... 83

Figure 49: Variation of sliding distance in P4C shaft hub contact region .................................. 83

Figure 50: Variation in torsional shear stress in P4C shaft...................................................... 83

Figure 51: Plot of contact status for P4C clearance fit connection........................................ 85

Figure 52: Loading for pure torsional load of $80 \mathrm{Nm}$ on P4C shaft (hub not shown)................. 87

Figure 53: Loading for torsional bending load on P4C shaft (hub not shown) ......................... 88

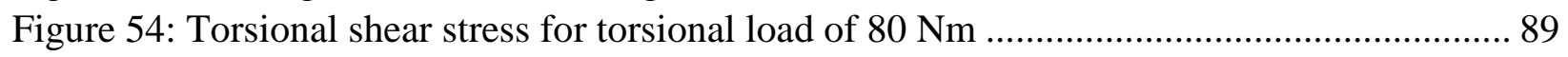

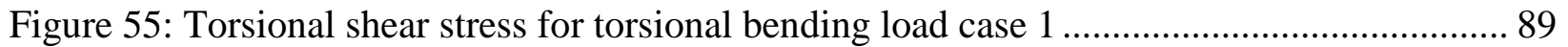

Figure 56: Torsional shear stress for torsional bending load case 2 ....................................... 90

Figure 57: Normal stress in axial direction at the middle of the shaft subjected to torsional load91

Figure 58: Normal stress in axial direction at the middle of the shaft subjected to torsional

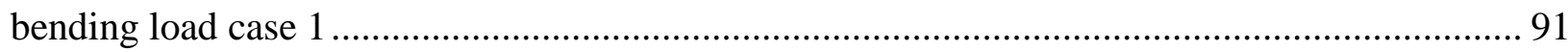

Figure 59: Normal stress in axial direction at the middle of the shaft subjected to torsional

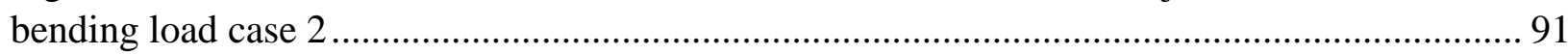

Figure 60: Maximum Axial displacement of hub (mm) for loading case 1............................ 92

Figure 61: Maximum Axial displacement of hub (mm) for loading case 2.............................. 92

Figure 62: Maximum contact stress for P3G and P4C shafts subjected to pure torsional load.... 93

Figure 63: Maximum contact stress for P3G and P4C shafts subjected to torsional bending load

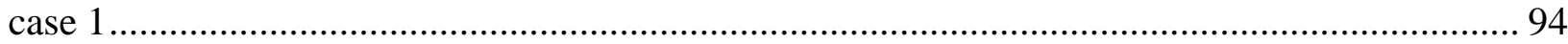

Figure 64: Maximum contact stress for P3G and P4C shafts subjected to torsional bending load

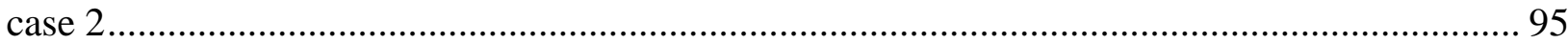
Figure 65: Average contact stress for P3G and P4C shafts subjected to torsional bending load

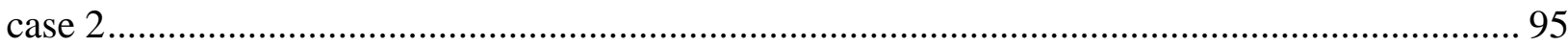




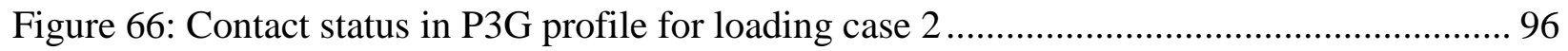

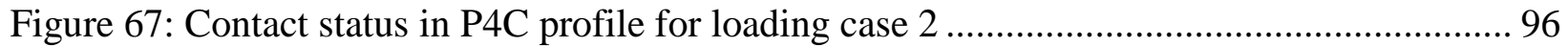

Figure 68: Contact Stress distribution for P3G profile for torsional load of $80 \mathrm{Nm}$.................. 98

Figure 69: Contact Stress distribution for P4C profile for torsional load of $80 \mathrm{Nm}$.................. 99

Figure 70: Contact Stress distribution for P3G clearance fit for torsional load of $80 \mathrm{Nm}$ and

bending load of 4600 N..................................................................................................... 100

Figure 71: Contact Stress distribution for P3G transition fit for torsional load of $80 \mathrm{Nm}$ and

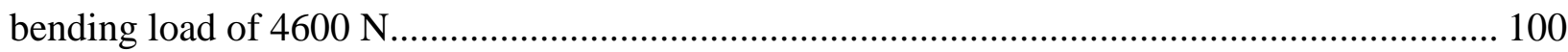

Figure 72: Contact Stress distribution for P3G interference fit for torsional load of $80 \mathrm{Nm}$ and

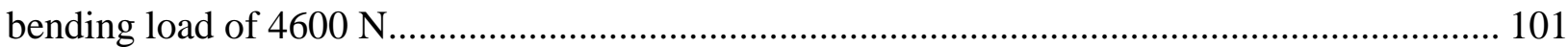

Figure 73: Contact Stress distribution for P4C clearance fit for torsional load of $80 \mathrm{Nm}$ and

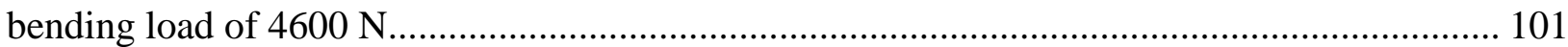

Figure 74: Contact Stress distribution for P4C transition fit for torsional load of $80 \mathrm{Nm}$ and bending load of 4600 N.............................................................................................. 102 Figure 75: Contact Stress distribution for P4C interference fit for torsional load of $80 \mathrm{Nm}$ and

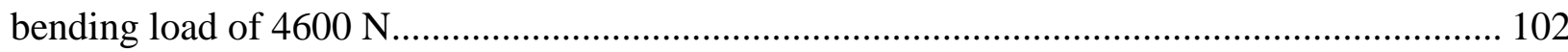

Figure 76: Maximum von Mises stress distribution for three and four lobe polygonal shaft subjected to pure torsional loading ..... 103

Figure 77: Maximum von Mises stress distribution for three and four lobe shaft subjected to

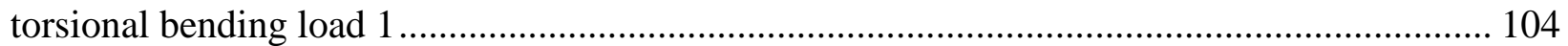

Figure 78: Maximum von Mises stress distribution for three and four lobe polygonal shaft

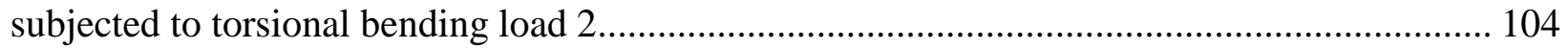
Figure 79: Position of maximum von Mises stress in P3G shaft for loading case 2 ................. 105 Figure 80: Position of maximum von Mises stress in P4C shaft for loading case 2................. 106 Figure 81: Maximum von Mises stress distribution for three and four lobe hub subjected to torsional load 107

Figure 82: Maximum von Mises stress distribution for three and four lobe hub subjected to torsional bending load case 1 .

Figure 83: Maximum von Mises stress distribution for three and four lobe hub subjected to torsional bending load case 2 .

Figure 84: Loading used for determining effect of friction in P3G (left) and P4C (right) connection.... 109

Figure 85: von Mises stress distribution in P3G hub with (left) and without (right) friction..... 110 Figure 86: von Mises stress distribution in P4C hub with (left) and without (right) friction ..... 110 Figure 87: von Mises stress distribution in P3G shaft with (left) and without (right) friction ... 111 Figure 88: von Mises stress distribution in P4C shaft with (left) and without (right) friction ... 111 Figure 89: Contact status in P3G and P4C connection with (left) and without (right) friction.. 111 Figure 90: Contact Stress in P3G connection with (left) and without (right) friction ............... 112 Figure 91: Contact stress in P4C connection with (left) and without (right) friction ................ 112 Figure 92: Resultant shear stress with (left) and without (right) friction ............................... 113 Figure 93: Total displacement (mm) in P3G connection with (left) and without (right) friction113 Figure 94: Total displacement (mm) in P4C connection with (left) and without (right) friction 114 Figure 95: Contact stress variation in P3G shaft with (left) and without (right) friction ........... 114 
Figure 96: Contact stress variation in P4C shaft with (left) and without (right) friction........... 114

Figure B1: Cross section of P4C shaft.............................................. 123

Figure B2: Loading for finite element analysis....................................... 124

Figure C1: Schematic of experimental loading..........................................128

Figure C2: Orientation for finding the von Mises stress................................ 129

Figure D1: Torsional bending loading for P3G connection.............................. 131

Figure D2: von Mises stress in P3G hub for torsional bending load............................131

Figure D3: von Mises stress in P3G shaft for torsional bending load..........................131

Figure D4: Contact stress in the P3G shaft along the edge for torsional bending load ...............131

Figure D5: Total deformation for torsional bending load in P3G shaft......................132

Figure D6: Torsional shear stress for torsional bending load in P3G shaft.....................132

Figure D7: Shear stress in xz direction for torsional bending load in P3G shaft................132

Figure D8: Shear stress in yz direction for torsional bending load in P3G shaft.......................132

Figure D9: Torsional bending loading for P4C connection...............................133

Figure D10: von Mises stress in P4C hub for torsional bending load........................133

Figure D11: von Mises stress in P4C shaft for torsional bending load.......................133

Figure D12: Contact stress in the P4C shaft along edge for torsional bending load............. 133

Figure D13: Total deformation for torsional bending load in P4C profile......................134

Figure D14: Torsional shear stress for torsional bending load in P4C shaft.....................134

Figure D15: Shear stress in xz direction for torsional bending load in P4C shaft...............134

Figure D16: Shear stress in yz direction for torsional bending load in P4C shaft................134

Figure D17: Torsional loading for P3G connection..................................... 135

Figure D18: von Mises stress in P3G hub for torsional load......................................135

Figure D19: von Mises stress in P3G shaft for torsional load...............................135

Figure D20: Contact stress in the P3G shaft along the edge for torsi.onal load..................135

Figure D21: Total deformation for torsional load in P3G profile...........................136

Figure D22: Torsional shear stress for torsional load in P3G shaft.............................136

Figure D23: Shear stress in xz direction for torsional load in P3G shaft......................136

Figure D24: Shear stress in yz direction for torsional load in P3G shaft.......................136

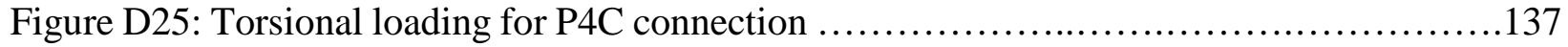

Figure D26: von Mises stress in P4C hub for torsional load...............................137

Figure D27: von Mises stress in P4C shaft for torsional load...................................137

Figure D28: Contact stress in the P4C shaft along the edge for torsional load..........................137

Figure D29: Total deformation for torsional load in P4C profile.............................138

Figure D30: Torsional shear stress for torsional load in P4C shaft..........................138

Figure D31: Shear stress in xz direction for torsional load in P4C shaft......................138

Figure D32: Shear stress in yz direction for torsional load in P4C shaft.....................138 


\section{List of Tables}

Table 1 : Main profile parameters of the German DIN polygon standards (2009-03) ................ 20

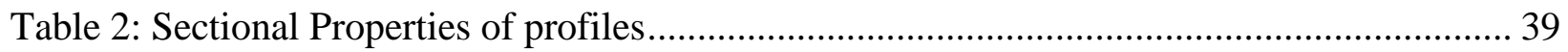

Table 3: Dimension of the profile for various fits ................................................................ 43

Table 4: Performance of various mesh elements ...................................................................... 50

Table 5: Table showing the effect of Newton Raphson option in solution ............................... 53

Table 6: Comparison of FEA and DIN standard for torsional loading of $80 \mathrm{Nm}$...................... 54

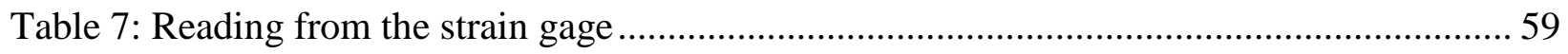

Table 8: Table showing the comparison of FEA and experimental results ............................... 60

Table 9: Comparison of analytical contact point determination to the FEA result. ................... 76

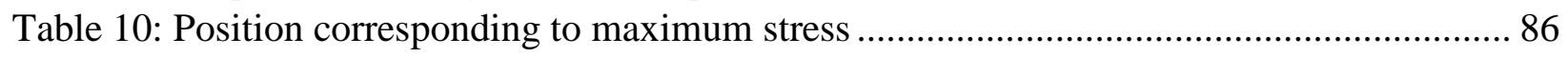

Table 11: Summary of result from friction analysis ........................................................... 115

Table D1: Result of loading on P3G shaft for pure torsional loading of $80 \mathrm{Nm}$...................139

Table D2: Result of loading on P4C shaft for pure torsional loading of 80 ...................139

Table D3: Result of P3G and P4C loading for loading case 1 for clearance fit...................140

Table D4: Result of P3G and P4C loading for loading case 2 for clearance fit.....................140

Table D5: Result of P3G and P4C loading for loading case 1 for transition fit..................141

Table D6: Result of P3G and P4C loading for loading case 2 for transition fit...................141

Table D7: Result of P3G and P4C loading for loading case 1 for interference fit................142

Table D8: Result of P3G and P4C loading for loading case 2 for interference fit...............142

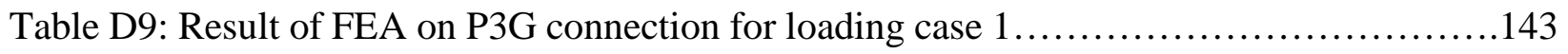

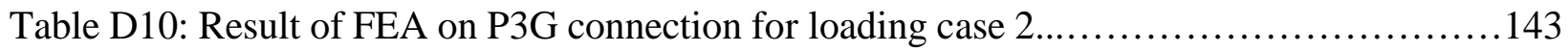

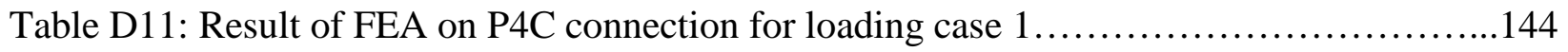

Table D12: Result of FEA on P4C connection for loading case 2............................144 


\section{Key to Symbols}

\begin{tabular}{|c|c|}
\hline Symbol & Descriptions \\
\hline$\phi(v)$ & Angle made at the center by parameter $v$ \\
\hline$v_{1}$ & Equation parameter for hub \\
\hline$v_{2}$ & Equation parameter for shaft \\
\hline$\phi_{1}$ & Angle made at the center by parameter $v$ of hub \\
\hline$\phi_{2}$ & Angle made at the center by parameter $v$ of shaft \\
\hline A & Cross sectional area \\
\hline $\mathrm{C}_{\mathrm{d}}$ & Difference in shaft to hub diameter \\
\hline $\mathrm{d}_{1}$ & Outer or grinding diameter \\
\hline $\mathrm{D}_{1}$ & Nominal diameter of hub \\
\hline $\mathrm{d}_{2}$ & Inside diameter \\
\hline $\mathrm{D}_{2}$ & Nominal diameter of shaft \\
\hline $\mathrm{D}_{\mathrm{m}}$ & Nominal Diameter \\
\hline$d_{n}$ & Damping coefficient in normal direction \\
\hline$d_{r}$ & Theoretical diameter \\
\hline $\mathrm{d}_{\mathrm{t}}$ & Damping coefficient in tangential direction \\
\hline e & Eccentricity \\
\hline E & Young's Modulus of Elasticity \\
\hline $\mathrm{e}_{1}$ & Eccentricity of hub \\
\hline $\mathrm{e}_{2}$ & Eccentricity of shaft \\
\hline $\mathrm{e}_{\text {lim }}$ & Limit of eccentricity \\
\hline $\mathrm{e}_{\mathrm{r}}$ & Theoretical eccentricity \\
\hline $\mathrm{f}$ & Distance of tangential force from center \\
\hline $\mathrm{F}$ & Force Matrix \\
\hline $\mathrm{F}_{\mathrm{NP}}$ & Profile Normal force \\
\hline $\mathrm{F}_{\mathrm{RG}}$ & Radial force from gear \\
\hline $\mathrm{F}_{\mathrm{TG}}$ & Tangential force from gear \\
\hline $\mathrm{F}_{\mathrm{TP}}$ & Profile Tangential force \\
\hline G & Modulus of Rigidity \\
\hline I & Moment of Inertia about neutral axis \\
\hline $\mathrm{I}_{\mathrm{p}}$ & Polar moment of inertia \\
\hline $\mathrm{I}_{\mathrm{x}}$ & Moment of inertia about $\mathrm{x}$ \\
\hline $\mathrm{K}$ & Stiffness matrix \\
\hline$l$ & width of hub \\
\hline $\mathrm{L}$ & Length of shaft between supports \\
\hline M & Bending Moment \\
\hline $\mathrm{n}$ & Number of lobes or periodicity \\
\hline
\end{tabular}




$\begin{array}{ll}\mathrm{N} & \text { Distance of normal force from center } \\ \mathrm{P} & \text { Contact pressure } \\ \mathrm{R} & \text { stationary radius of epitrochoidal curve } \\ \mathrm{r} & \text { rolling radius of epitrochoidal curve } \\ \mathrm{r}(v) & \text { Radius of curvature } \\ \mathrm{r}\left(v_{1}\right) & \text { Radius of curvature of hub } \\ \mathrm{r}\left(v_{2}\right) & \text { Radius of curvature of shaft } \\ \mathrm{t} & \text { Hub wall thickness } \\ \mathrm{T} & \text { Torque } \\ \mathrm{T}_{\mathrm{max}} & \text { Maximum Torque } \\ \mathrm{U} & \text { Displacement matrix } \\ v & \text { Parameter for drawing polygonal profile } \\ v_{1} & \text { Parameter for drawing polygonal profile for hub } \\ v_{2} & \text { Parameter for drawing polygonal profile for shaft } \\ \mathrm{v} & \text { Poisson Ratio } \\ \mathrm{Z}_{\mathrm{p}} & \text { Polar moment of resistance } \\ \beta & \text { Contact or pressure angle } \\ \beta_{1} & \text { Pressure angle for hub } \\ \beta_{2} & \text { Pressure angle for shaft } \\ \varepsilon & \text { Ratio of eccentricity of hub to shaft } \\ \theta & \text { Parameter for drawing epitrochoidal curve } \\ \mu & \text { Coefficient of friction } \\ \sigma & \text { Allowable tensile stress } \\ \sigma_{\mathrm{x}} & \text { Normal stress along x axis } \\ \sigma_{\mathrm{y}} & \text { Normal stress along y axis } \\ \sigma_{\mathrm{z}} & \text { Normal stress along z axis } \\ \tau & \text { Torsional shear strength } \\ \tau_{\mathrm{max}} & \text { Maximum Shear stress } \\ \tau_{\mathrm{xy}} & \text { Shear force in xy plane } \\ \tau_{\mathrm{yx}} & \text { Shear force in yz plane } \\ \tau_{\mathrm{zx}} & \text { Shear force in zx plane } \\ & \end{array}$




\section{Abbreviations}

$\begin{array}{ll}\text { Abbreviations } & \text { Descriptions } \\ \text { 2D } & \text { Two Dimensional } \\ \text { 3D } & \text { Three Dimensional } \\ \text { ASM } & \text { American Society of Metals } \\ \text { CNC } & \text { Computerized Numerical Control } \\ \text { DIN } & \text { Deutsches Institut für Normung (German standard) } \\ \text { DOF } & \text { Degree of freedom } \\ \text { FEA } & \text { Finite Element Analysis } \\ \text { FEM } & \text { Finite Element Method } \\ \text { P3G } & \text { Three lobe polygon profile in accordance with DIN } 32711 \\ \text { P4C } & \text { Four lobe polygon profile in accordance with DIN } 32712\end{array}$




\section{Chapter 1: Introduction}

\section{1) Introduction}

Multi lobe shaft and hub connections were used during the ancient times to turn wooden wheels and gears. The modern industrial use for power transmission was dominated by splines and keyed shafts due to their ease in manufacturing and design. Owing to the complex geometry, lack of theoretical solution of the complex tri-axial stress state, difficulty in manufacturing from traditional methods and higher cost to keyways, the polygonal shafts were not as popular as keys and splines. With the advent of modern manufacturing such as multi axis CNC milling and grinding and numerical methods as FEM, these issues have been addressed and the use of polygonal connections is increasing. They are used in the automobile industry (Citarella and Gerbino, 2001) and heavy machines such as those used in coal mines, large scale reducers and shifters for rolling machine tools (Lü \& Liu, 2011). The polygonal connections have advantages as lower cost of manufacturing (40-50 \% as compared to the spline joint), no stress risers as in keyways and splines, self-centering connection, and less vibration and noise as the connection can work in shrink fit unlike keyed shafts (Huang, Li, Sun \& Wang, 2010).

Among the various polygonal profiles, three lobe (P3G) and four lobe (P4C) polygonal profiles as standardized by DIN, are commonly used in industries. Usually, four lobed shafts are used in sliding fit application and three lobed polygonal shafts are used in in press fit applications for torque transmission. Past studies on polygonal shafts and hubs have focused on a particular type of profile and its comparison to the splines. Studies have been conducted on polygonal profiles of same nominal diameter with different outer diameter for different geometric parameters for finding the optimized shape without the inter-comparison of different profiles. Similar sized three and four lobe polygonal connection, as standardized by DIN 
standard, have different load carrying capacities. A comparison needs to be made between the load carrying capacity of the two profiles from a manufacturing point of view i.e. the same outer grinding diameter at various fits. This study is focused on studying the comparative loading strength of these two types of polygonal profiles at various fits from a manufacturing point of view.

\section{2) Purpose}

The primary purpose of this study is to compare the load carrying capacity of three lobe and four lobe polygonal profile shafts and hubs of a constant grinding diameter at various fits. To achieve this primary purpose, following are the secondary purposes of the research:

i) To compare the stress developed in the three lobe and four lobe polygonal shaft and hub connection manufactured from the same grinding diameter subjected to torsional bending load at various fits

ii) To compare the results of FEA model to the experimental value and DIN standard

iii) To find an analytical shear stress distribution and point of contact for three lobe and four lobe polygonal shaft subjected to torsional load and use this result to verify FEA results.

iv) To determine the effect of friction in loading strength

v) To determine the contact stress distribution at various fits for torsional and torsional bending load

\section{3) Scope}

The study analyses the loading strength of three and four lobe polygonal shafts subjected to a torsional bending load, manufactured from the same grinding diameter and do not compare the shafts based on their nominal sizes 


\section{4) Assumptions}

The assumptions considered for the research depended on the part of analysis. For the finite element analysis, assumptions were that the material is uniform with ideal boundary conditions and ideal geometry as depicted by beam theory. For the analytical portion, assumptions were the assumptions of Saint Venant Torsion principle explained in section 4.4.

\section{5) Hypothesis or Research Question}

The P3G shafts are favored for greater power transmission in interference fit and the P4C shafts are favored for low torque sliding fit applications. The research question is to either refute or accept this use for equal grinding diameter sized P3G and P4C sized shafts for torsional bending loads.

\section{6) Significance}

The results from the research will help to understand the performance of the polygonal shafts under various fits and suitability of a particular profile and fit for a particular application.

\section{7) Theory}

\subsection{1) Geometry of Polygonal Profile}

Mathematically, the polygonal profile is a special case of an epitrochoidal curve. It is the locus of a point $\mathrm{P}$ at a distance of ' $\mathrm{e}$ ' from center of a circle of radius ' $\mathrm{r}$ ' that rolls over a circle of radius ' $\mathrm{R}$ ' without slipping. The combination of $\mathrm{R}, \mathrm{r}$, and, e can generate an infinite number of epitrochoidal curves, but only the curves with a whole numbered ratio of $\mathrm{R}$ to $\mathrm{r}$ generate a closed curve. If there are no concave branches, the profile is known as K- profile and can be described by parametric equations, in Cartesian or in Polar coordinates. The parametric equations for the curves are:

$$
\mathrm{x}=(\mathrm{R}+\mathrm{r}) \cos (\theta)-\operatorname{ecos}\left(\frac{(\mathrm{R}+\mathrm{r}) \theta}{\mathrm{r}}\right)
$$




$$
y=(R+r) \sin (\theta)-\operatorname{esin}\left(\frac{(R+r) \theta}{r}\right)
$$

Figure 1 shows effect of $r$ and e on the shape of the curve using equations (1) and (2).

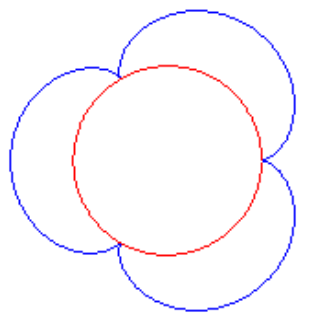

$\mathrm{R}=15, \mathrm{r}=5$, $\mathrm{e}=5$

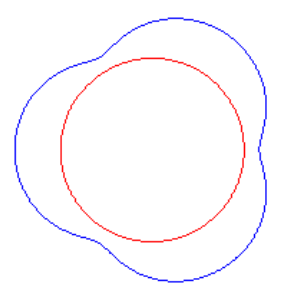

$\mathrm{R}=15, \mathrm{r}=5, \mathrm{e}=2.5$

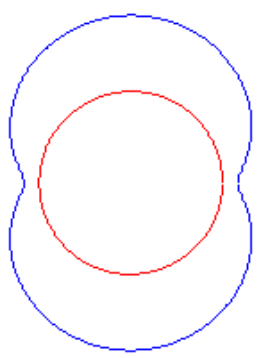

$\mathrm{R}=15, \mathrm{r}=7.5, \mathrm{e}=5$

Figure 1: Common Epitrochoidal Curves drawn using equations (1) and (2)

In an epitrochoidal curve, higher value of eccentricity generates sharper corners. The ratio of $\mathrm{R}$ to $\mathrm{r}$, also known as periodicity, gives the number of corners or lobes in the curve, as evident from Figure 1. For the polygonal shaft and hub, the geometry of the epitrochoidal curve can be described in parametric form as shown in Equations (3) through (6).

Cartesian Form of Equation:

$$
\begin{gathered}
x(v)=\left(\left(\frac{D_{m}}{2}-e \cos (n v)\right) \cos (v)-n e \sin (n v) \sin (v)\right) \\
y(v)=\left(\left(\frac{D_{m}}{2}-e \cos (n v)\right) \sin (v)+n e \sin (n v) \cos (v)\right)
\end{gathered}
$$

Polar Form of Equation:

$$
\left.\begin{array}{c}
r(v)=\sqrt{\left(\frac{D_{m}}{2}-e \cos (n v)\right)^{2}+(n e \sin (n v))^{2}} \\
\phi(v)=v+\tan ^{-1}\left(\frac{n e \sin (n v)}{0.5 D_{m}-e \cos (n v)}\right)
\end{array}\right]
$$

where, $D_{m}$ is the mean diameter of the profile, $\mathrm{e}$ is the eccentricity, $\mathrm{n}$ is the periodicity (number of lobes), $v$ is the parameter, $0 \leq v \leq 2 \pi$.

The expression for radius of curvature of random points on a polygonal profile is given in terms of $\mathrm{x}$ and $\mathrm{y}$ as: 


$$
r(v)=\frac{\left(\dot{x}^{2}+\dot{y}^{2}\right)^{1.5}}{|\ddot{x} \dot{y}-\ddot{y} \dot{x}|}
$$

where, the single dot represents derivative with respect to $v$ and double dot represents double derivative with respect to $v$.

The expressions from equation (3) through (5) can be seen from the geometric description of a three lobe polygonal profile from Figure 2 for drawing an epitrochoidal curve.

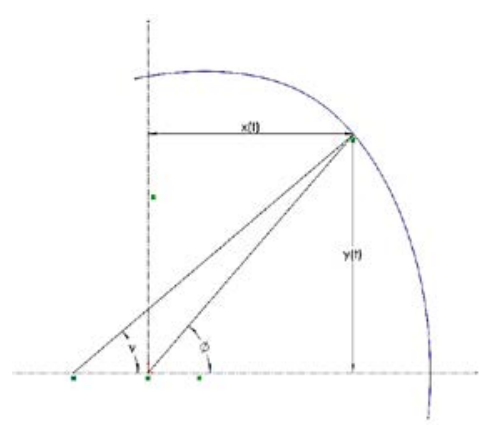

Figure 2: Geometrical Description of a three lobe polygon curve

To get a closed and non-intersecting shape for making shafts, the limit is given for eccentricity value as:

$$
\mathrm{e}_{\lim }=\frac{\mathrm{D}_{\mathrm{m}}}{2\left(\mathrm{n}^{2}-1\right)}
$$

Some of the common harmonic polygonal profiles are shown in Figure 3.

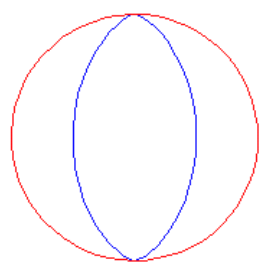

Two lobe

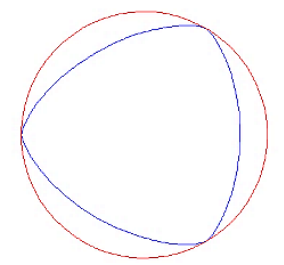

Three lobe

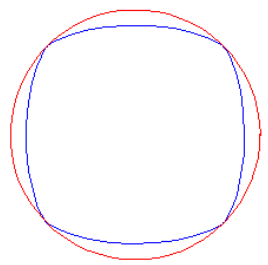

Four lobe

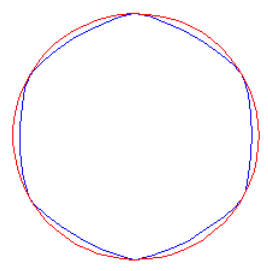

Six lobe

Figure 3: Different Polygonal profiles 


\subsection{2) DIN 32711 and 32712 standard}

Three and four lobed polygonal profiles are extensively used polygonal profiles in industries and are standardized by DIN standard. The three lobe profile is a harmonic curve as described by equation (3), while the four lobe profile is the superimposition of the four lobe profile as described by equation (3) and circle with diameter as the grinding diameter, as shown on the top of Table 1.

Table 1 : Main profile parameters of the German DIN polygon standards (2009-03)

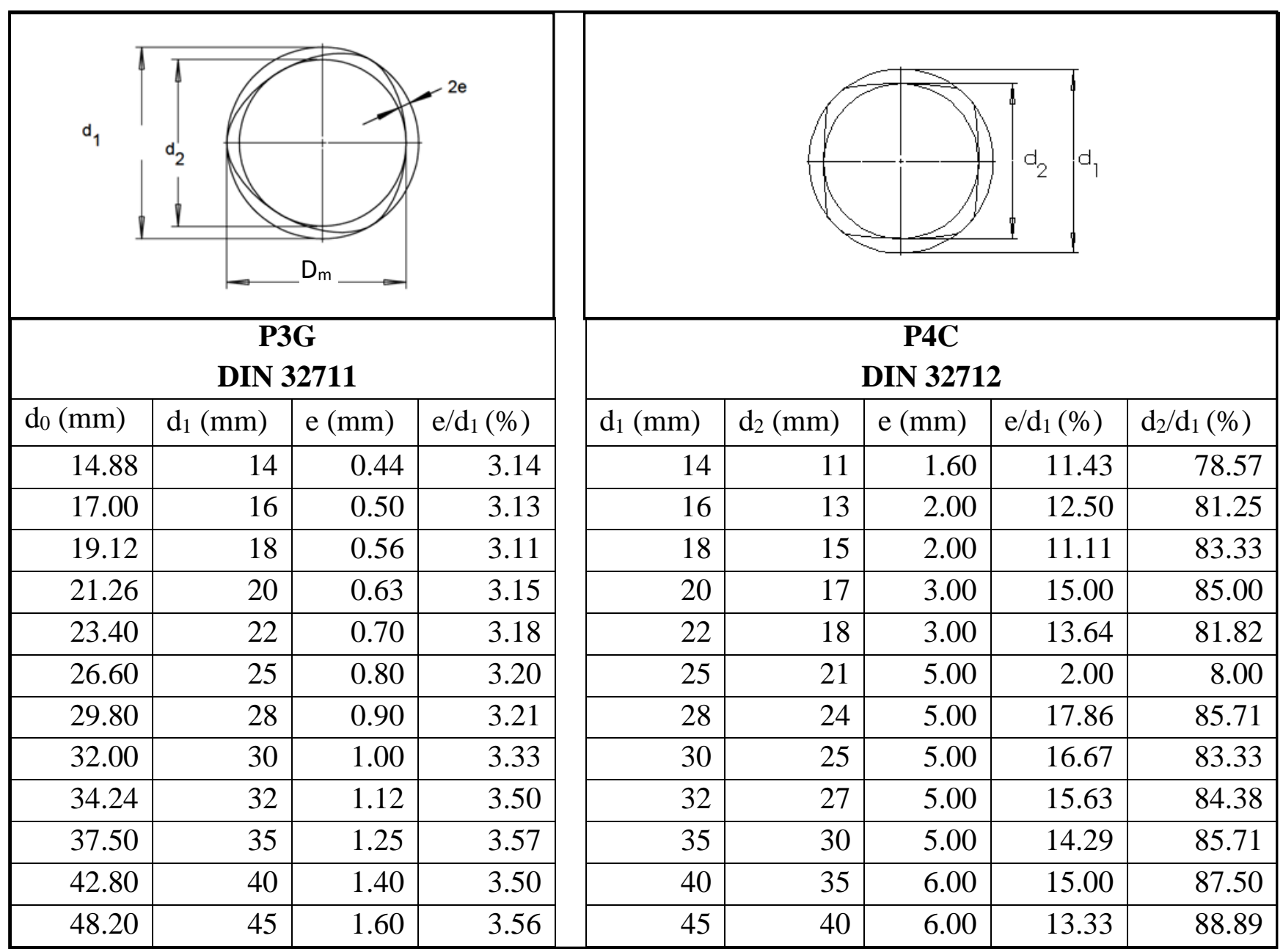

DIN standard 32711 (2009-03) describes the geometry and stress calculation for three lobe P3G polygonal profile and DIN standard 32712 (2009-03) describes the geometry and stress 
calculation for four lobe P4C polygonal profile. The P3G shaft hub connection is described by the nominal diameter $D_{m}=d_{1}$, eccentricity e, inside diameter $d_{2}$ and related eccentricity $\frac{e}{d_{1}}$. The P4C shaft hub connection is described by grinding diameter $d_{1}$, the inside diameter $d_{2}$ and ratio $\frac{d_{1}}{d_{2}}$ is used for defining the shaft and hub profiles as shown in Table 1 . The nominal diameter of four lobe is given by $\mathrm{D}_{\mathrm{m}}=\mathrm{d}_{2}+2 \mathrm{e}$.

The polygonal shafts manufactured according to DIN standard with parameters as shown in Table 1 are designed according to the maximum shear stress and contact pressure determined by DIN standard for a pure torsional loading case. The calculation for geometric parameters and the stresses are as follows.

For P3G profile

$$
\text { Maximum Torque }\left(\mathrm{T}_{\max }\right)=\tau \times \mathrm{Z}_{\mathrm{p}}
$$

where, $\tau$ is the torsional shear stress

$$
\text { Polar moment of resistance }\left(Z_{p}\right)=\frac{d_{1}+4 e}{d_{1}+8 e} \frac{A^{4}}{20 I_{p} d_{1}}
$$

where, $d_{1}$ is the mean diameter,

$$
\begin{array}{r}
\text { Area }(A)=\frac{\pi d_{1}{ }^{2}}{4}-4 \pi e^{2} \\
\text { Polar Moment of Inertia }\left(I_{p}\right)=\frac{\pi d_{1}{ }^{4}}{32}-\frac{3 \pi d_{1}{ }^{2} e^{2}}{4}-6 \pi e^{4} \\
\text { Moment of Inertia about } x \text { axis, }\left(I_{x}\right)=\frac{I_{p}}{2}
\end{array}
$$

Thickness (Distance between the end of profile and the outer circumference of hub)

$$
\begin{aligned}
& \text { Thickness for under } 35 \mathrm{~mm} \text { mean diameter }(\mathrm{t})=1.44 \times \sqrt{\frac{\mathrm{T}_{\max }}{\sigma \times l}} \\
& \text { Thickness for over } 35 \mathrm{~mm} \text { mean diameter }(\mathrm{t})=1.2 \times \sqrt{\frac{\mathrm{T}_{\max }}{\sigma \times l}}
\end{aligned}
$$

where, $l$ is the width of the hub and $\sigma$ is the allowable tensile stress 
Surface Pressure:

$$
\mathrm{P}=\frac{\mathrm{T}_{\max }}{\mathrm{l}\left(\operatorname{\pi ed}_{1}+\frac{\mathrm{d}_{1}^{2}}{20}\right)}
$$

Similarly, for P4C Profile

Shaft:

$$
\begin{array}{r}
\text { Maximum Torque }\left(\mathrm{T}_{\max }\right)=\tau \times \mathrm{Z}_{\mathrm{p}} \\
\text { Polar Moment of Resistance }\left(\mathrm{Z}_{\mathrm{p}}\right)=0.2 \mathrm{~d}_{2}{ }^{3}
\end{array}
$$

Hub:

$$
\text { Thickness }(t)=0.7 \times \sqrt{\frac{T_{\max }}{\sigma \times 1}}
$$

Surface Pressure:

$$
\mathrm{P}=\frac{\mathrm{T}_{\max }}{\mathrm{l}\left(\pi \mathrm{e}_{\mathrm{r}} \mathrm{d}_{\mathrm{r}}+\frac{\mathrm{d}_{\mathrm{r}}^{2}}{20}\right)}
$$

where, $\tau$ is the torsional shear stress, $d_{2}$ is the inner diameter, $d_{r}=d_{2}+2 e$ is the calculated theoretical diameter, e is the eccentricity, $\mathrm{d}_{1}$ is the diameter of circumscribed circle, $l$ is the width of the hub, $\sigma$ is the allowable tensile stress.

$$
\text { Theoretical eccentricity }\left(e_{r}\right)=\frac{d_{1}-d_{2}}{4}
$$

\subsection{3) Forces on Polygonal Shaft Connections}

The polygonal shaft is a form fit connection that transmits power by form contact and does not suffer from stress concentration issues unlike keys and splines. For a torque transmission from a spur gear to the polygonal shaft with the help of a central hub, the forces are as shown in Figure 4. The central bending load and the torsion will be applied from the hub (hub not shown in figure). 


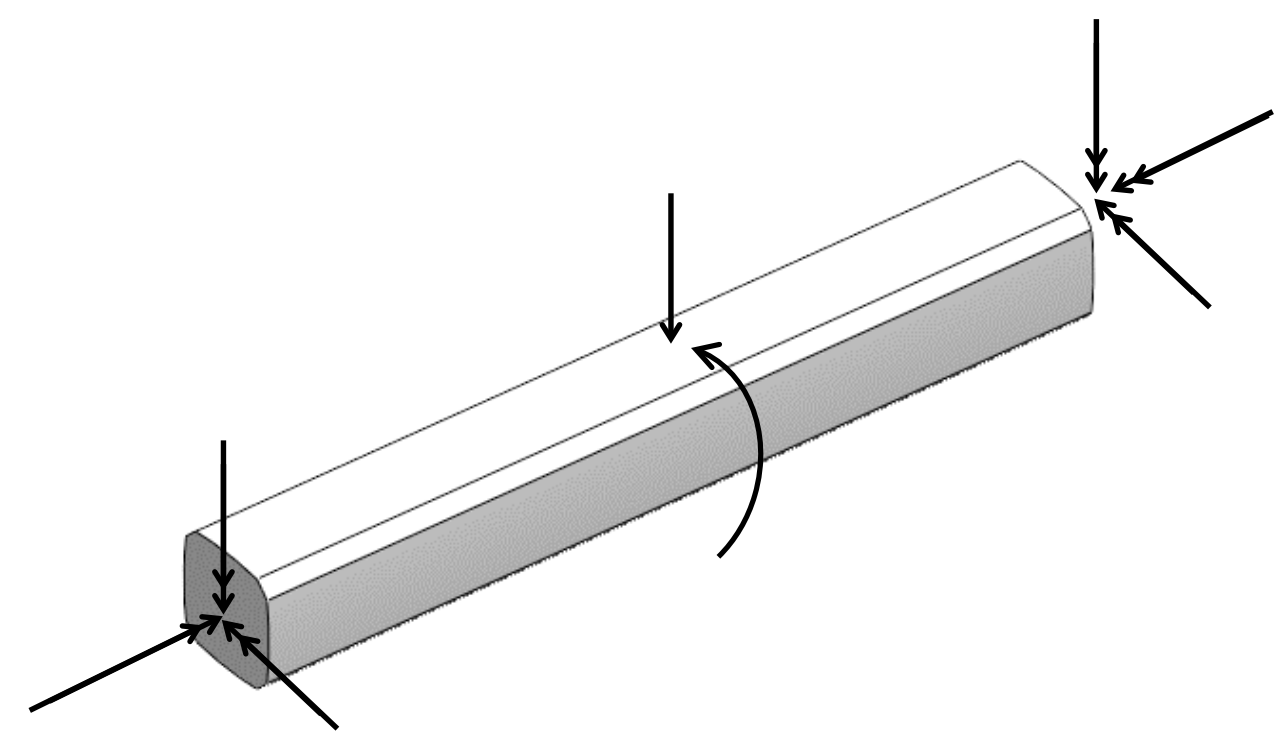

Figure 4: Loading condition of shaft

Assuming an idealized condition of point contact at a point $\mathrm{P}$ on the profile as shown in Figure 5 and 6 , the forces can be determined with the outer profile representing hub and the inner profile representing shaft. Referring to Figure 5 and 6, for a position $\mathrm{P}$ on the surface at a polar coordinates ( $\mathrm{r}, \phi)$, the force acting at the point $\mathrm{P}$ in the shaft-hub connection can be expressed as two orthogonal components, $\mathrm{F}_{\mathrm{TP}}$ (tangent force on profile) and $\mathrm{F}_{\mathrm{NP}}$ (normal force on profile) with their lever arms $\mathrm{f}$ and $\mathrm{N}$. Considering the drive from a gear, the resultant force of $\mathrm{F}_{\mathrm{TP}}$ and $\mathrm{F}_{\mathrm{NP}}$ is equal to the tangential force, $\mathrm{F}_{\mathrm{TG}}$ from gear.

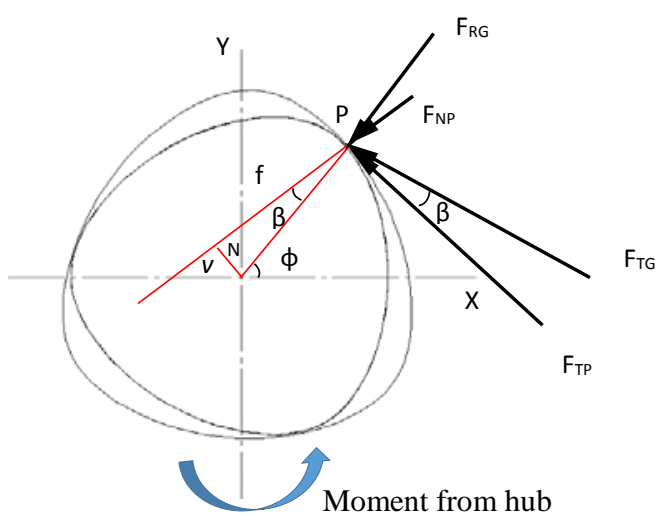

Figure 5: Forces in a P3G shaft hub connection for idealized point of contact 


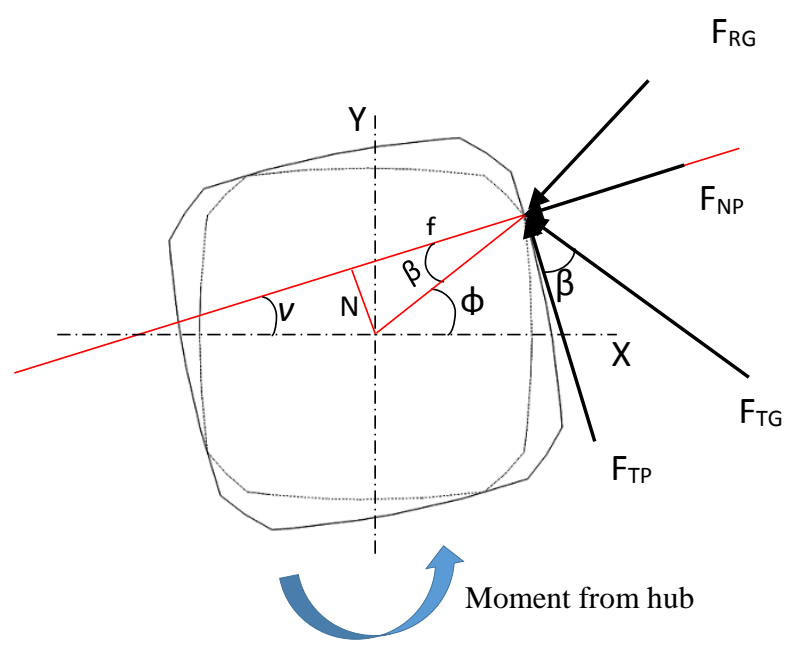

Figure 6: Forces in a P4C shaft hub connection for idealized point of contact

The normal to the surface at the point P makes an angle $\beta$ with the position vector $\mathrm{r}(\phi)$, which is known as the contact angle. The contact angle for a parametric equation in $v$ is given as:

$$
\tan \beta(v)=\frac{(\mathrm{n} \text { e } \sin (n v))}{\left(\frac{D_{m}}{2}-e \cos (n v)\right)}
$$

The variation in $\beta$ determines the relation between the normal force and the tangential force transmission since $\tan (\beta)$ is equal to the ratio of $\mathrm{N}$ to $\mathrm{f}$. For the same resultant force acting on the profile, lower values of $\beta$ result in higher tangential forces and lower normal forces. Most of the torque is transmitted by the tangential force. In the case of a press fit, $\beta=0$, the torque is transmitted by tangential force only and is equal to $\mu \mathrm{F}_{\mathrm{NP}}$ where $\mu$ is the coefficient of friction between the shaft and the hub.

For the torque transmission from the hub attached to a spur gear with $20^{\circ}$ pressure angle, the forces at point $\mathrm{P}$ are

$$
\begin{array}{ll}
\text { Radial force due to bending: } & \mathrm{F}_{\mathrm{RG}}=\mathrm{F}_{\mathrm{TG}} \tan \left(20^{\circ}\right) \\
\text { Normal force due to contact: } & \mathrm{F}_{\mathrm{NP}}=\mathrm{F}_{\mathrm{TG}} \sin (\beta) \\
\text { Frictional force: } & \mathrm{F}_{\mathrm{TP}}=\mathrm{F}_{\mathrm{TG}} \cos (\beta)
\end{array}
$$


where, $F_{T G}$ is the tangential force from gear, $F_{R G}$ is radial force from the gear, $F_{T P}$ is the tangential force on profile and $\beta$ is the contact angle at the point of idealized contact.

Considering a section of the element in the contact region where the resultant stress is maximum, the body is subjected to the tensile stress in the $\mathrm{z}$ direction i.e. along the axis $\left(\sigma_{\mathrm{z}}\right)$ and torsional shear stress ( $\tau_{\mathrm{zx}}$ and $\left.\tau_{\mathrm{yz}}\right)$. The combined effect of these forces can be evaluated to calculate the loading capacity of the system using distortion energy theorem. There is no analytical proof for determining the conformal contact force and the local shear stress in the section, which leads to rigid assumptions.

The angle of contact, $\beta$, acts as a determinant of the relative frictional and normal force. For a constant torque transmitted, the torque is given as:

$$
\begin{array}{r}
\text { Torque }=F_{T G} R=F_{T P} f+F_{N P} N \\
\text { Torque }=\left(F_{T G} \cos \beta\right) f+\left(F_{T G} \sin \beta\right) N
\end{array}
$$

For a constant torque, as the value of $\beta$ increases, the profile tangential force is lower and the normal force is higher. For lower $\beta$, most of the torque is carried by the profile tangential force as $\mathrm{N} \rightarrow 0$ and normal force does not transmit torque. Neglecting the movement in the axial direction due to warping of the noncircular shaft, the effect of friction can be evaluated. The magnitude of profile tangential force relative to the frictional force can cause the body to either slide or stick. The higher pressure angle will have higher normal force and subsequently higher friction force, which will lead to lesser slip in the circumferential direction. This should be verified by a relatively higher contact pressure in the case of P4C and lesser slip than the P3G connection and higher stresses in lower frictional coefficient connections. 


\subsection{4) Point of Contact}

The stress analysis of the shaft and hub is nonlinear due to the contact mechanics and is even more complicated by the conformal contact of the shaft and the hub.

Although, there is conformal contact over a region in an isometric three lobe polygonal shaft, for a clearance fit, the contact regions tends to be smaller and mathematically in the ideal case can be approximated by a point contact. For a point of contact $\mathrm{P}$, as shown in Figures 7 and 8 for P3G and P4C connection, the angle $\phi$ corresponding to this point of contact can be determined mathematically.

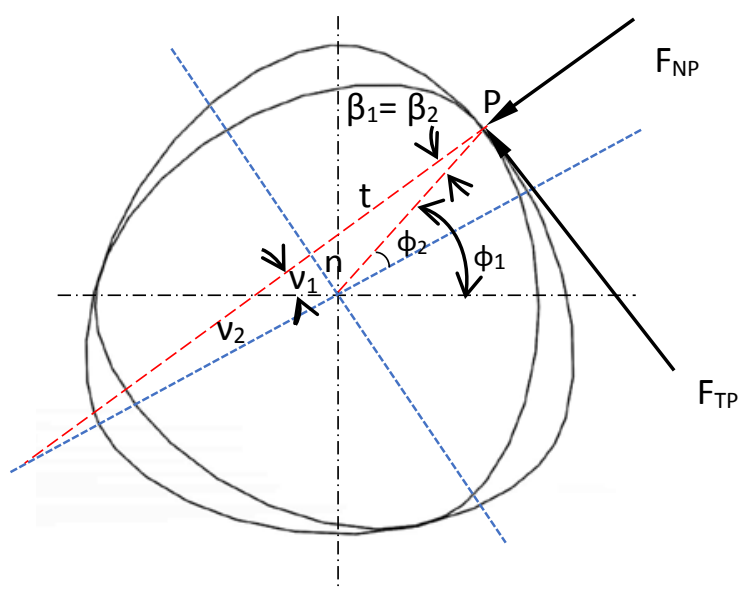

Figure 7: Point of contact in P3G profile

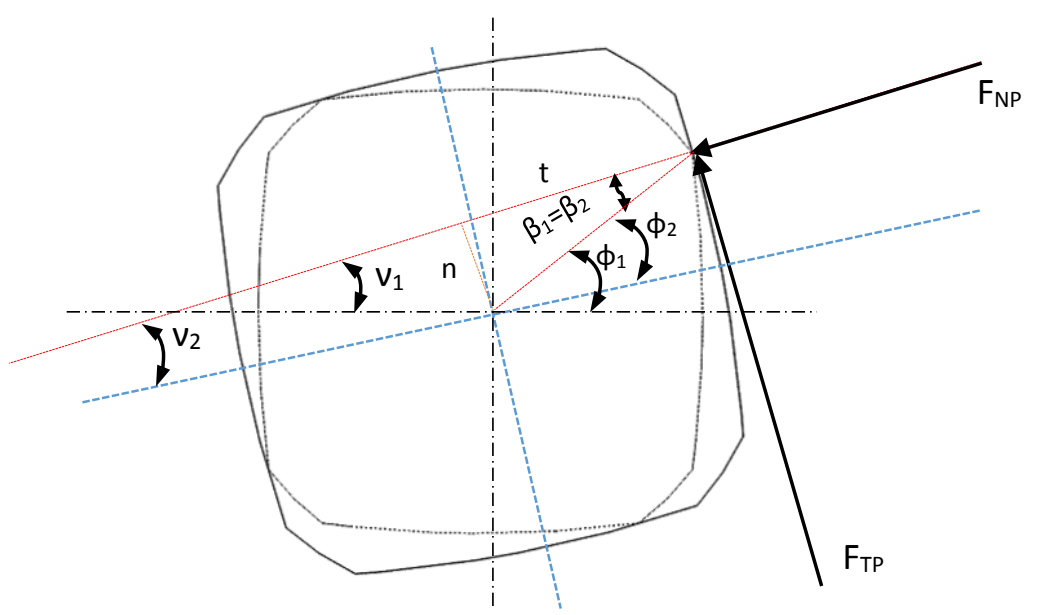

Figure 8: Point of contact in P4C profile 
Referring to Figures 7 and 8, the outer profile is the hub and inner profile is the shaft.

For P3G profile as shown in Figure 7, the point where the hub and the shaft meet, radius of curvature and the angle of contact are the same for the shaft and the hub. Using equations (4) and (20),

For the hub:

$$
\begin{array}{r}
r\left(v_{1}\right)=\sqrt{\left(\frac{D_{1}}{2}-e_{1} \cos \left(n v_{1}\right)\right)^{2}+\left(n e_{1} \sin \left(n v_{1}\right)\right)^{2}} \\
\left.\beta_{1}=\tan ^{-1}\left(\frac{n e_{1} \sin \left(n \cdot v_{1}\right)}{\left(\frac{D_{1}}{2}-e_{1} \cos \left(n \cdot v_{1}\right)\right.}\right)\right)
\end{array}
$$

For the shaft:

$$
\begin{array}{r}
r\left(v_{2}\right)=\sqrt{\left(\frac{D_{2}}{2}-e_{2} \cos \left(n v_{2}\right)\right)^{2}+\left(n e_{2} \sin \left(n v_{2}\right)\right)^{2}} \\
\beta_{2}=\tan ^{-1}\left(\frac{\mathrm{n} \mathrm{e}_{2} \sin \left(n \cdot v_{2}\right)}{\left.\left(\frac{D_{1}-e_{2} \cos \left(n \cdot v_{2}\right)}{2}\right)\right)}\right.
\end{array}
$$

where, $r$ is the radius of curvature, $\beta$ is the angle of contact

For condition of $r\left(v_{1}\right)=r\left(v_{2}\right)$,

$$
\left(\frac{D_{1}}{2}-e_{1} \cos \left(n v_{1}\right)\right)^{2}+\left(n e_{1} \sin \left(n v_{1}\right)\right)^{2}=\left(\frac{D_{2}}{2}-e_{2} \cos \left(n v_{2}\right)\right)^{2}+\left(n e_{2} \sin \left(n v_{2}\right)\right)^{2}
$$

Also, $\beta_{1}=\beta_{2}$

$$
\left.\left.\left(\frac{n e_{1} \sin \left(n \cdot v_{1}\right)}{\left(\frac{D_{1}}{2}-e_{1} \cos \left(n \cdot v_{1}\right)\right.}\right)\right)=\left(\frac{n e_{2} \sin \left(n \cdot v_{2}\right)}{\left(\frac{D_{2}}{2}-e_{2} \cos \left(n \cdot v_{2}\right)\right.}\right)\right)
$$


Solving equations (30) and (31)

$$
\left(\frac{D_{1}}{2}-e_{1} \cos \left(n v_{1}\right)\right)^{2}\left(1+\left(\frac{n e_{2} \sin \left(n v_{2}\right)}{\frac{D_{2}}{2}-e_{2} \cos \left(n v_{2}\right)}\right)^{2}\right)=\left(\frac{D_{2}}{2}-e_{2} \cos \left(n v_{2}\right)\right)^{2}\left(1+\left(\frac{n e_{1} \sin \left(n v_{1}\right)}{\frac{D_{1}}{2}-e_{1} \cos \left(n v_{1}\right)}\right)^{2}\right)
$$

Placing the ratio value from equation (31)

$$
\left(\frac{\mathrm{D}_{1}}{2}-\mathrm{e}_{1} \cos \left(\mathrm{n} v_{1}\right)\right)=\left(\frac{\mathrm{D}_{2}}{2}-\mathrm{e}_{2} \cos \left(\mathrm{n} v_{2}\right)\right)
$$

From equations (31) and (32),

$$
\begin{aligned}
n e_{1} \sin \left(n v_{1}\right)=n e_{2} \sin \left(n v_{2}\right) \\
\sin \left(n v_{2}\right)=\varepsilon \sin \left(n v_{1}\right)
\end{aligned}
$$

where, $\varepsilon=\frac{e_{1}}{e_{2}}$

Also from equation (32),

$$
\cos \left(n v_{2}\right)=\frac{\left(\frac{\mathrm{C}_{\mathrm{d}}}{2}+\mathrm{e}_{1} \cos \left(\mathrm{n} v_{1}\right)\right)}{\mathrm{e}_{2}}
$$

where, $C_{d}=D_{2}-D_{1}$

Squaring and adding equations (33) and (34),

$$
\begin{aligned}
1 & =\varepsilon^{2}+\left(\frac{\mathrm{C}_{\mathrm{d}}}{2 \mathrm{e}_{2}}\right)^{2}+2 \frac{\mathrm{C}_{\mathrm{d}} \varepsilon}{2 \mathrm{e}_{2}} \cos \left(\mathrm{n} v_{1}\right) \\
\cos \left(\mathrm{n} v_{1}\right) & =\left(\frac{1-\varepsilon^{2}-\left(\frac{\mathrm{C}_{\mathrm{d}}}{2 \mathrm{e}_{2}}\right)^{2}}{\mathrm{C}_{\mathrm{d}} \varepsilon}\right) \mathrm{e}_{2}
\end{aligned}
$$




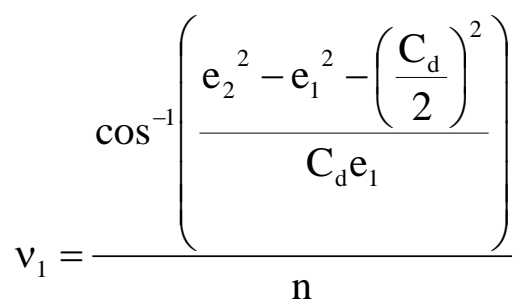

Similarly,

$$
\cos \left(n v_{2}\right)=\frac{\left(\frac{C_{d}}{2}+e_{1} \frac{e_{2}{ }^{2}-e_{1}{ }^{2}-\left(\frac{C_{d}}{2}\right)^{2}}{C_{d} e_{1}}\right)}{\cos ^{-1}\left(\frac{\mathrm{C}_{d}}{2 e_{2}}+e_{1} \frac{1-\varepsilon^{2}-\left(\frac{C_{d}}{2 e_{2}}\right)^{2}}{C_{d}}\right)}
$$

From equation (35) and (36), the point of contact can be calculated, which can be used to determine the pressure angle at the point of contact and associated forces.

For the conditions where the value of $C_{d}\left(D_{2}-D_{1}\right)$ is zero i.e. for line fit, the value of $v_{1}$ and $v_{2}$ are:

$$
v_{1}=v_{2}=\frac{\cos ^{-1}(\infty)}{n}
$$

Equation 37 is true, since theoretically for a line-to-line contact all the points on the hub should touch all the points on the shaft and a single value of $v$ would not be possible. Also, for a non-harmonic profile as a P4C profile, the point of contact may be a point that may not exist in the truncated curve and need numerical methods to solve it. 


\section{Chapter 2: Review of Literature}

The modern engineering use of polygonal shafts was limited, due to complex profile geometry that needed dedicated grinding machines, compared to the ease to produce alternatives such as splined and keyed shafts until the development of numerically controlled machining. In 1939, a kinematically controlled grinding machine (Maximov, 2005) for the production of triangular profiles with filleted corners, called K- profiles (named after the company KrauseVienna, which introduced this type of joint (Huang et al, 2010)), was developed. One of the early studies on these profiles was conducted in Germany in 1958 by E. Filemon (1959). In the 1960s, epicycloidal profiles with three and four lobes were first made and the DIN standard 32711 for three lobe (P3G profile) and 32712 for four lobe (P4C profile) polygonal profiles were published in 1979 (Maximov,2005). Polygonal shafts still had limited use due to difficulty in manufacturing until the advent of modern manufacturing techniques and processing. Using computer numerically controlled manufacturing, the polygonal shafts can be precisely and economically manufactured and is being used in industries due to its advantages.

\section{1) Analytical Stress Analysis}

Stress analyses of polygonal profiles were developed by various writers such as Orlov, Leroy, Viseur, Musyl and Manhurim (Citarella \& Gerbino, 2005) and were based on very strong approximations that are not able to clarify the real stress and strain state. The aim of the analysis was to find the critical stress in the hub since hub was supposed to expand under torsion and fail. The procedure attempted to simplify the geometry of the polygon connection by analogous mechanical models. For example, Musyl used circular segments for profile approximation. This approach of Musyl is referred by the polygonal connection manufacturers (Taylor, 1987). The current DIN standard is also developed on the works of Musyl and provides an approximation of 
contact pressure and to compute nominal stress state for static torsional loads (Standard DIN 32711 \& 32712, 2009-03). Later, Ziaei calculated the stress state using conformal mapping for a shaft subjected to torsion (as cited in Grossman, 2006). All these analyses did not consider the more realistic loading condition of torsional bending. In the absence of an accurate analytical solution of the polygonal shaft, numerical methods have been used to determine the loading stress.

\section{2) Numerical Stress Analysis}

Numerical studies on the polygonal shaft hub connection were performed using the finite element method and the boundary element method. The numerical analysis conducted in polygonal connections has been explained in two sections; viz. 2D stress analysis and 3D stress analysis.

\subsection{1) 2D Stress Analysis}

Due to the complex conformal nature of the contact between the shaft and the hub in a polygonal connection, significant work in the contact pressure distribution in polygonal shaft and hub connections was not conducted until the advent of numerical methods. The contact being conformal, does not fall under Hertz contact theory and has to depend upon numerical solution. With the popularity of numerical methods, more realistic stress and strain analyses in polygonal shafts were developed. The initial numerical studies by Braschel et al. (as cited in Grossman, 2007) and Czyzewski and Odman (1988) were restricted to the two dimensional analysis that omitted the possibility of stress concentration at the end of the hub contact. Czyzewski and Odman (1988) did an analysis on contact stress and deformation on trilobe polygonal connections, which was the first published solution to the contact problem in trilobe connection (Czyzewski \& Odman, 1988). The study consisted of an in-plane torsional loading without 
considering the friction and bending moment and showed the effect of torque and clearance on the contact pressure. The result found that the contact pressure is approximately triangular in distribution and does not resemble Hertz contact theorem, being a highly conformal contact. Czyzewski and Odman (1988) recommended the value of maximum shear stress for determining the load capacity of the connection. The effect of torsional loading and diametrical clearance on contact pressure distribution found from the study are shown in Figure 9.

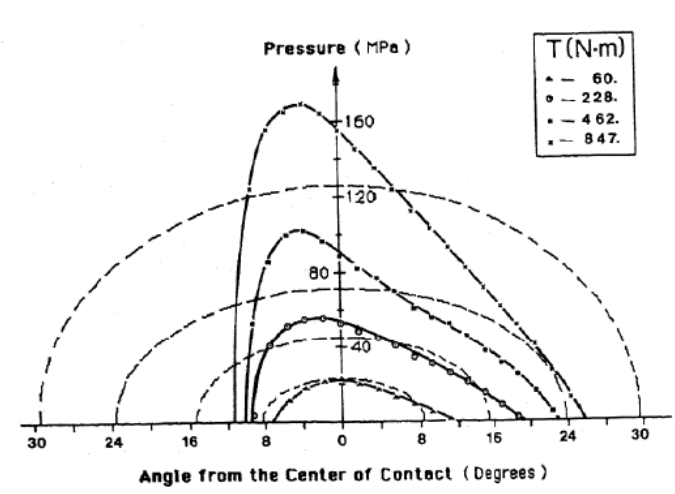

(a)

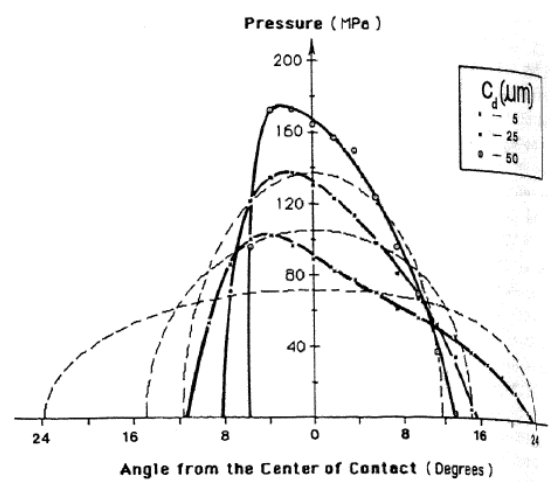

(b)

Figure 9: Effect of (a) Torsional loading and (b) Diametrical Clearance on pressure distribution in zone of contact (Czyzewski \& Odman, 1988).

(Permission to reproduce image is in Appendix E)

Huang et al. (2010) studied the effect of friction on contact stress in a trilobe connection. Similar to the studies conducted by Czyzewski and Odman (1988), Huang et al. (2010) found the triangular distribution of contact stress and found that friction decreases the value of the normal contact stress, although the distribution and rule of contact stress were unaffected. They also found an increase in contact area and decrease in the normal contact force as the eccentricity increased. The analysis of shearing stress in polygonal shaft and hub was conducted by Lü and Liu (2011) using plane stress analysis for concentrated load at three points in trilobe polygonal shaft and hub connection and found higher shear stress in hub than the shaft. 
The comparison of trilobe polygonal shafts to the involute splines for shafts with mean diameter of the polygonal shaft equal to the pitch diameter of the involute splines were performed by Kahn-Jetter, Hundertmark and Wright (2000) and found tensile stress in the polygonal connection to be significantly lower than in the spline due to the lack of stress concentration. This showed a positive trait for using polygonal connections. These studies were only two dimensional and did not depict the realistic behavior of the connection and the need for three dimensional study was inevitable.

\subsection{2) 3D Stress Analysis}

Mechnik conducted a three dimensional analysis, mainly on three lobe polygonal profiles, and found the peak stress at the edge of the hub and shaft connection. This study was mainly concentrated on the effect of the shape of the polygon on stress values and suggested increased profile eccentricity for better load carrying capacity (as cited in Grossman, 2007). Citarella and Gerbino (2001) used boundary element analysis to determine the state of stress and strain, which came in close conformance to the finite element results of Mechnik, but with lesser computation than FEA. The study of Gottlichar was a more realistic three dimensional study, since it accounted for the bending load, which is inevitable in most of the transmission. This showed the shaft as the most endangered part due to fretting corrosion exposure (as cited in Grossman, 2007). Grossman (2007) used the torsional bending load for both three and four lobe polygonal connections and looked at the most optimized shape in terms of loading capacity. He found that the global profile shape determined the fatigue strength of the connection more than the manufacturing precision. 


\section{3) Conformal Contact Problems}

The highly conformal geometry of a polygonal shaft and hub excludes the necessary assumptions to be analyzed by Hertzian contact theory. A contact is said to be conforming if the surfaces of the two bodies 'fit' exactly or even closely together without deformation as in polygonal shafts. The nature of contact is a closely conformal concentrated contact of convex and concave surfaces. Although asperities occur in these type of conformal contacts, such asperities are so small relative to the geometry that they can be neglected during the analysis (Grossman, 2007).

Due to the presence of the appreciable fraction of the circumference of the shaft and the hub in contact, the elastic half space theory cannot be applied. Also, the effect of friction cannot be neglected and the body tends to be outside the scope of Hertzian contact theory. In the limiting case where the clearance between the shaft and the hub is large, the concept of nonconformal contact can be used to approximate the solution.

To find the contact stress for Non-Hertzian elastic bodies, the analytical form needs initial separation to be described in simple quadratic form, which is not possible in the epitrochoidal curve. Hence, computational contact mechanics is applied to analyze the system.

\section{4) Computational Contact Mechanics}

The need of nonlinear analysis arises due to the dynamic stresses that occur because of changing contact areas (topology nonlinearity) for which there is no analytical solution. The stiffness of the structure is a function of displacement and is no longer constant and the solution is nonlinear.

The contact and target elements are to be defined where the contact approaches the target because the two bodies do not interpenetrate. To avoid the penetration, ANSYS ${ }^{\circledR}$ uses various 
methods of contact formulation. The pure penalty method uses a virtual spring that provides stiffness so that the body does not penetrate. Another contact formulation, the Normal Lagrange adds an extra degree of freedom, contact pressure, so as to satisfy the variational inequality. Combining the advantages of both these methods, Augmented Lagrangian formulation can be used, which defines the normal push back force as a combination of the normal stiffness and Lagrangian contact pressure as shown in equation (38):

$$
\mathrm{F}_{\mathrm{n}}=\mathrm{k}_{\mathrm{n}} \mathrm{x}_{\mathrm{n}}+\lambda
$$

where, $F_{n}$ is the normal push back force, $k_{n}$ is the stiffness and $x_{n}$ is the displacement of virtual spring from Penalty method, $\lambda$ is the contact pressure from Lagrangian formulation.

This method is less sensitive than the pure penalty method and is preferred for frictional contact stress problems (ANSYS INC, 2010). The way by which the computational software addresses non linearity is by dividing a load step into a number of time steps (sub steps), which have several iterations for each linearized equilibrium condition. If the solution do not converge in these sub steps, the bisection method is enabled to define new sub steps. Computational software such as ANSYS ${ }^{\circledR}$ uses the Newton Raphson method to solve the problem, which is a series of linear approximations with corrections. It shall be clearer from Figure 10, which shows the Newton Raphson method for a single sub step. The iterations continue for force and displacement convergence for a given criteria. The reason for iteration is the nonlinearity. In a nonlinear analysis, as in the contact problem, the stiffness is a function of displacement and is not constant. The equation is given as:

$$
\left[\mathrm{K}\left(\mathrm{U}_{\mathrm{i}}\right)\right]\left\{\mathrm{U}_{\mathrm{i}}\right\}=\left\{\mathrm{F}_{\mathrm{i}}\right\}
$$

where, $\mathrm{U}_{\mathrm{i}}$ and $\mathrm{F}_{\mathrm{i}}$ are the $\mathrm{i}^{\text {th }}$ displacement and force, where $\mathrm{i}$ is the current equilibrium iteration. 
Initially the displacement is at $\mathrm{U}_{\mathrm{o}}$ and the external force is $\mathrm{F}_{\mathrm{o}}$ and the point on the response curve is $P_{0}$. The software increases the external force by $F_{0}+\Delta F$ so that using the Jacobian or tangent stiffness, $\mathrm{K}\left(\mathrm{U}_{\mathrm{o}}\right)$, the displacement is calculated as:

$$
\left[\mathrm{K}\left(\mathrm{U}_{\mathrm{o}}\right)\right]\{\Delta \mathrm{U}\}=\{\Delta \mathrm{F}\}
$$

The displacement is increased to $\mathrm{U}_{1}=\mathrm{U}_{\mathrm{o}}+\Delta \mathrm{U}$ and the point is $\left(\mathrm{U}_{1}, \mathrm{~F}_{\mathrm{o}}+\Delta \mathrm{F}\right)$ in $\mathrm{P}^{\prime}$ as shown in Figure 10. Then, the value of displacement is substituted back in equation (39) to get the actual force $F_{1}$. The difference between the force, $F_{0}+\Delta F$ and $F_{1}$ is called residual. If the residual force is less than the criterion, the subset is said to be converged. Otherwise, the next iteration is carried on with the value of $\mathrm{U}_{1}, \mathrm{~F}_{1}$ as the new initial point.

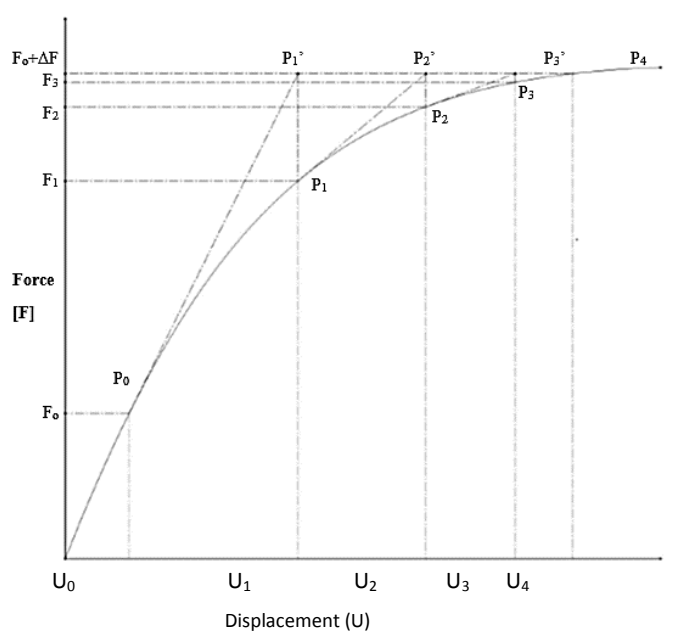

Figure 10: Newton Raphson method for solving nonlinear problems 


\section{Chapter 3: Methodology}

The purpose of this study is to compare the load carrying capacity of P3G and P4C profile shafts at various fits that can be manufactured from the same stock size. There is no analytical solution to the complex conformal contact of the polygonal connections and computational methods such as FEM is needed to achieve this goal. The methodology adopted consisted of performing a pilot Finite Element study using ANSYS 15.0.7 software on P3G and P4C profiles with the same grinding diameter and verifying the results to the experimental, analytical results and DIN standard and then analyze the verified finite element model at various fits. Since, the stress at conformal contact was not determined by other methods than FEA, the verification was performed with stress at other locations. The following describes the methodology adopted in detail.

\section{1) Selection of polygonal profile size}

The first step in comparing the two profiles was to find the correct size of P3G and P4C profile that can be grinded from the same stock size. For this purpose, the shaft of grinding diameter $15.875 \mathrm{~mm}$ was selected. The aim from the manufacturing point of view was to make the polygonal profile with the largest possible size from the given stock diameter. P4C maintained the outer diameter of $15.875 \mathrm{~mm}$, whereas the P3G needed some grinding on the outer profile to make the harmonic P3G profile, resulting in a slightly smaller outer diameter. The dimensions of the two profiles are as follows.

P3G profile:

Nominal diameter $\left(\mathrm{D}_{\mathrm{m}}\right)=14.478 \mathrm{~mm}$

Eccentricity $(\mathrm{e})=0.508 \mathrm{~mm}$

Eccentricity Limit $\left(\mathrm{e}_{\lim }\right)=\frac{\mathrm{D}_{\mathrm{m}}}{2\left(\mathrm{n}^{2}-1\right)}=0.904 \mathrm{~mm}$ 
where $n=3$, number of lobes.

The eccentricity limit is more than the eccentricity value of $0.508 \mathrm{~mm}$. So, these parameters result in a closed curve.

P4C profile:

Outer Diameter $\left(\mathrm{d}_{1}\right)=15.875 \mathrm{~mm}$

Inner Diameter $\left(\mathrm{d}_{2}\right)=13.335 \mathrm{~mm}$

Eccentricity (e) $=1.905 \mathrm{~mm}$

Nominal diameter $\left(\mathrm{D}_{\mathrm{m}}\right)=\mathrm{d}_{2}+2 \mathrm{e}=17.145 \mathrm{~mm}$

The dimensions of the P3G and P4C shaft and hub assembly used for FEA are shown in Figures 11 and 12. A larger diameter has been considered for the hub so as to eliminate any effect due to the torsional load on the outer profile on shaft hub interface.

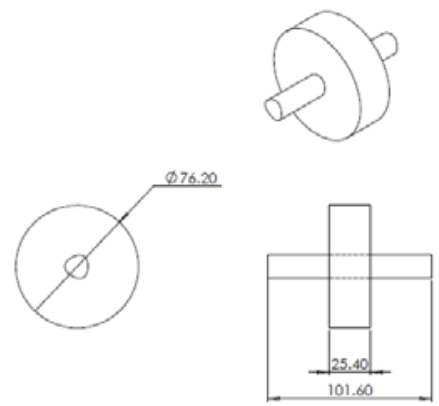

Figure 11: Dimension of the three lobe polygonal shaft and hub used for FEA in mm

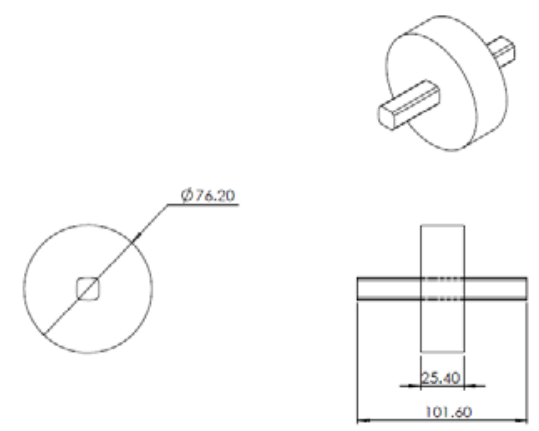

Figure 12: Dimension of the four lobe polygonal shaft and hub used for FEA in mm 
The difference in the cross section of the two profiles is shown in Figure 13, which shows similar area in the two profiles. Table 2 shows the difference in the area numerically and P4C profile has larger area and Moment of Inertia compared to P3G shafts.

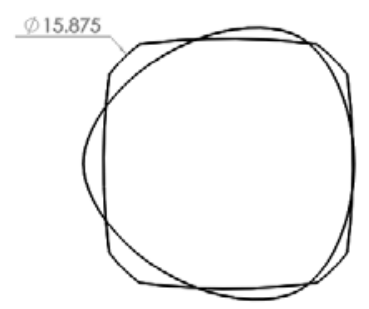

Figure 13: Comparison of cross section of P3G and P4C profile

Table 2: Sectional Properties of profiles

\begin{tabular}{|r|r|r|}
\hline Profile & Cross sectional area $\left.\mathbf{( m m}^{\mathbf{2}}\right)$ & Moment of Inertia $\mathbf{( m m}^{\mathbf{4}} \mathbf{)}$ \\
\hline P3G & 161.39 & 2092.39 \\
\hline P4C & 164.90 & 2201.71 \\
\hline
\end{tabular}

3.2) Loading in P3G and P4C profiles

The second step after finding the size of the profiles was to find the effect of the fit and the profile on the loading capacity. For example, a press fit has better profile conformance between the shaft and the hub, which in turn should cause a larger contact area and a smaller contact pressure as compared to the clearance fit, when subjected to the same torsion. There will, however, be added stress due to the interference itself. The P4C connection should give higher contact stress due to the presence of higher maximum contact angle (allowing smaller tangential force and greater normal force). All of these comparisons need to be made from the standpoint of the same grinding diameter. To evaluate these effects, P3G and P4C profiles were loaded for clearance, transitional and press fit for constant grinding diameter with torsional bending load in an FEA model. The results of the analyses were then compared for torsional shear stress, normal stress, hub displacement, contact stress, and von Mises stress for all fits. 
The result from a single static solution would not represent the critical stress in a full revolution as the polygonal connection is not symmetric with respect to the central axis. To cater this situation, a rotating bending load was applied over $120^{\circ}$ range for P3G and $90^{\circ}$ range for $\mathrm{P} 4 \mathrm{C}$ at $10^{\circ}$ step with $1 \mathrm{~s}$ ramped loading for each step. The loading conditions are shown in Figure 14 for P3G and Figure 15 for P4C connection. Only half of the connection was modelled and symmetry was applied to the middle face along axial direction. The first load step was modeled as a pure torsional load and the bending load was applied from the second load step. The bending load has been incorporated by a rotating radial force on the hub. This type of loading is close to the actual phenomena where the position of bending load varies with the rotation of the shaft. This is different than the loading of Grossman (2007), where the bending load was incorporated by a couple on either end of the hub so as to make pure bending condition in shaft hub connection and omit the effect of the shear force. The loading used here has transverse shear force applied as well and is closer to the industrial loading, although the effects of transverse shear would be less for a longer shaft.

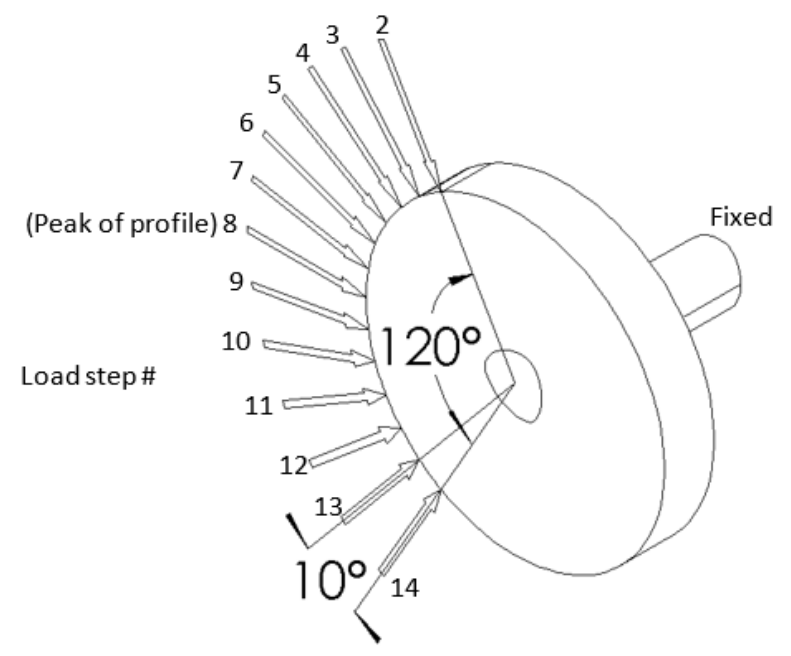

Figure 14: Bending Load in P3G shafts for FEA 


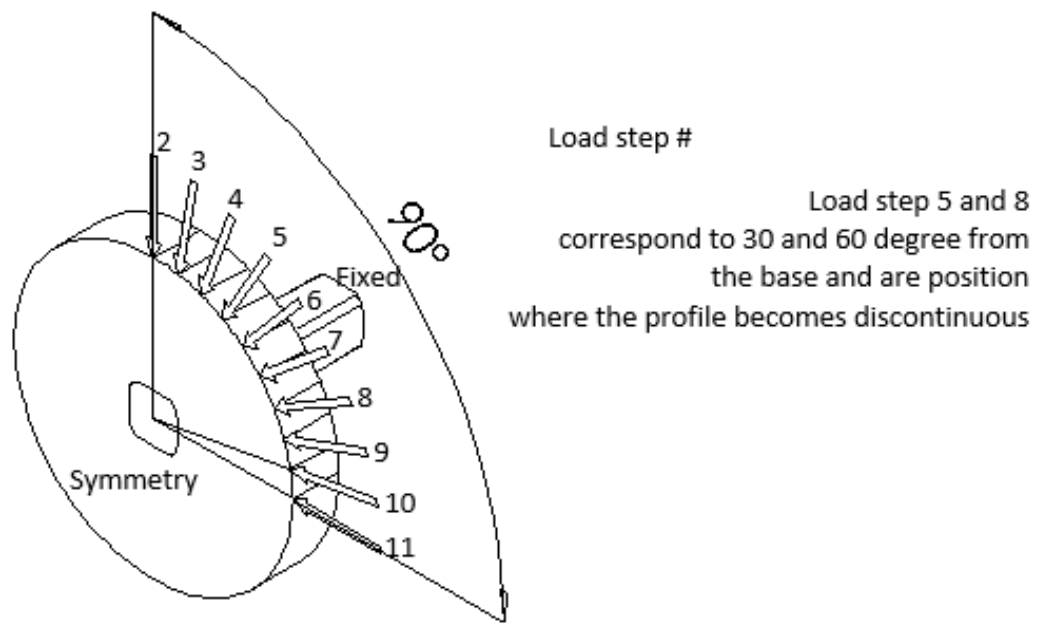

Figure 15: Bending Load in P4C shafts for FEA

The load step corresponding to maximum von Mises stress was considered for further analysis. While running the FEA for pure torsional load as per the dimension shown in Appendix A under pure torsion, it was found that one of the sides was experiencing a slightly magnitude of von Mises stress distribution from the other two. This was caused by lack of a purely symmetrical mesh and the multi-point contact method used by ANSYS to simulate torsional load. To avoid such situations and prevent warpage of the hub, the hub diameter was increased to $76.2 \mathrm{~mm}$ from $38.1 \mathrm{~mm}$ and width of $25.4 \mathrm{~mm}$ as shown in Figures 11 and 12 . This led to a maximum difference of $1.77 \%$ in P4C and $1.5 \%$ in P3G for maximum von Mises stress between all periodic contact positions and was considerable for further analysis.

For calculating the load to be applied for FEA, an approximate stress determination was performed on the P4C shaft. The calculation is shown in Appendix B. The result found from the P4C shaft has been used in both of the profiles for uniformity in load. The result has been calculated for a small load so as to avoid local yield. The practical use of the polygonal shaft and hub will be in much higher stresses and local yielding will occur. To study this, another set of loads has also been considered for the analysis by increasing the load magnitude using similar 
calculations. The following analyses were performed to analyze the effect of various magnitude of forces on the connection:

i) Pure torsional load of $80 \mathrm{Nm}$ using structural steel (ASTM A36 hot rolled) of Yield stress $250 \mathrm{MPa}$.

ii) Torsional Bending of a torsional load of $8 \mathrm{Nm}$ with $410 \mathrm{~N}$ radial bending force (no local yield using 4140 steel oil quenched of Yield stress of $965 \mathrm{MPa}$ ). This steel has been used to see the effect without any yielding.

iii) Torsional bending of a torsional load of $80 \mathrm{Nm}$ with $4600 \mathrm{~N}$ radial bending force (local yielding using structural steel of Yield stress $250 \mathrm{MPa}$ )

The reason for choosing structural steel in the higher load case is to see the effect on the connection after local plastic deformation and the reason for choosing oil quenched 4140 steel in lower loading case is to avoid any local yielding, even in the worst case scenario, although the manufacturers use 4140 cold finished and annealed steel per ASM 6382.

\section{3) Pilot Finite Element Analysis}

A pilot study was conducted initially and verified experimentally, analytically and from DIN standard. The major considerations while applying the finite element method were as follows:

\subsection{1) Geometric Consideration}

For the purpose of analysis, the shaft and hub assembly was modeled with the hub at the center of the shaft. Only one-half of the assembly, along the shaft axis, was considered and the rest was taken as symmetrical as shown in Figures 14 and 15. 


\subsection{2) Fit Consideration}

Six different configurations of the polygonal shaft and hub connections were analyzed, which were clearance, transition and interference fit for P3G and P4C profiles. The clearance fit was H8/f7, which is the close running fit. The interference fit was H7/p6, which is the locational interference fit. The transition fit was a line-to-line fit. The dimensions of the shaft and hub are shown in Table 3.

Table 3: Dimension of the profile for various fits

\begin{tabular}{|c|c|c|c|c|c|c|c|c|c|c|}
\hline \multirow[b]{2}{*}{ 応 } & \multirow[b]{2}{*}{ 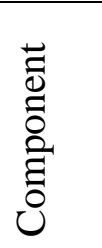 } & \multicolumn{3}{|l|}{ P3G } & \multicolumn{5}{|l|}{ P4C } & \multirow[b]{2}{*}{ 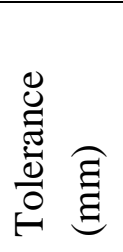 } \\
\hline & & 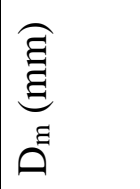 & $\underbrace{\underset{\Xi}{\Xi}}_{0}$ & $\stackrel{\xi}{\circledR}$ & 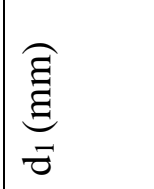 & $\underset{\text { త్ర్తి }}{\underset{\Xi}{\Xi}}$ & $\underbrace{\underset{\Xi}{\Xi}}_{0}$ & $\vec{\theta}$ & ت্তি & \\
\hline \multirow{2}{*}{ 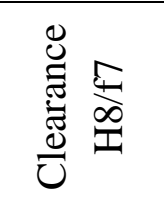 } & Shaft & 14.445 & 0.508 & 0.0352 & 15.842 & 13.302 & 1.905 & 0.1203 & 0.8397 & 0.058 \\
\hline & Hub & 14.503 & 0.508 & 0.0350 & 15.900 & 13.360 & 1.905 & 0.1198 & 0.8403 & \\
\hline \multirow{2}{*}{ 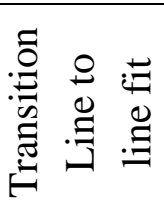 } & Shaft & 14.478 & 0.508 & 0.0351 & 15.875 & 13.335 & 1.905 & 0.1200 & 0.8400 & 0 \\
\hline & Hub & 14.478 & 0.508 & 0.0351 & 15.875 & 13.335 & 1.905 & 0.1200 & 0.8400 & \\
\hline \multirow{2}{*}{ 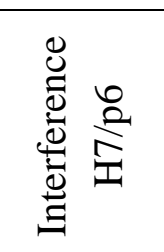 } & Shaft & 14.496 & 0.508 & 0.0350 & 15.893 & 13.353 & 1.905 & 0.1199 & 0.8402 & 0.018 \\
\hline & Hub & 14.478 & 0.508 & 0.0351 & 15.875 & 13.335 & 1.905 & 0.1200 & 0.8400 & \\
\hline
\end{tabular}

\subsection{3) Non Linear Consideration}

The surface interaction between the polygonal shaft and hub at their contact should be considered to avoid the interpenetration of the components. The surface-to-surface contact was chosen since the area of contact is unknown. Other nonlinear considerations were:

i) The contact solution formulation chosen was Augmented Lagrangian that takes into consideration the advantages of both penalty and Lagrangian methods 
ii) The number of iterations was set to $100(\mathrm{NEQIT}=100)$ with default convergence criteria $(0.5 \%)$

iii) The contact behavior was chosen as symmetrical, which meant that neither the target nor the contact can penetrate each other

iv) The shaft was considered as contact being the convex part and the hub was considered as target being the concave part (“Introduction of Contact ANSYS Mechanical Structural Nonlinearities,” 2010).

v) For the clearance fit, where the hub was free, the stabilization damping factor was set to 0.2 to avoid rigid body motion

vi) The nonlinear solution was run with Augmented Lagrangian with the force, displacement convergence criterion and line search parameter enabled.

\subsection{4) Transient Consideration}

The transient structural analysis was performed using static torsion and superimposed rotating bending since the point of application of bending load is varying along the circumference with respect to the rotation of the connection. The rotating bending was performed for $120^{\circ}$ range for $\mathrm{P} 3 \mathrm{G}$ and $90^{\circ}$ range for $\mathrm{P} 4 \mathrm{C}$ at $10^{\circ}$ step with $1 \mathrm{~s}$ ramped loading for each step with 10 to 20 sub steps for each load step.

\subsection{5) Friction Consideration}

The previous studies concluded that friction does not change the solution state and only decreases the tangential contact stress. These analyses did not consider bending load and warpage and were only two-dimensional in modeling. Friction has been considered in this

analysis, in the torsion and the torsional bending load cases to simulate the actual loading 
condition. The coefficient of friction was taken as 0.18 from the studies of Grossman (2007). To find the effect of friction, the analysis has been performed with and without friction.

\subsection{6) Meshing consideration}

Any accurate finite element solution is highly dependent on the correct choice of element and the art of good meshing. The mesh elements initially chosen were higher order hexahedral (with mid nodes), lower order hexahedral (without mid nodes) and higher order tetrahedral elements (with mid nodes). Higher order hexahedral elements are known to converge faster while lower order hexahedral and higher order tetrahedral elements need finer mesh for similar convergence. Traditionally, lower order hexahedral mesh were used in contact pressure problems, as it can be refined to a finer mesh with a lower number of nodes and elements and give reasonable accuracy. With the increasing higher process computing, this is no longer the case and all type of mesh can be used. Especially for complicated geometry, the tetrahedral mesh provides the added advantage of better conformity to the geometry and higher order hexahedral mesh converge faster.

A desirable mesh is the one in which decreasing the mesh size no longer changes the solution value. If the mesh is of low quality, the convergence can be misleading as values will converge to some other inaccurate magnitudes. This is common in contact problems, where the solution may converge to undesirable values. To achieve an accurate solution, the individual mesh convergence for a type of element and the inter comparison between converged solution of each element type were considered. Each mesh was checked for quality before simulation using the following guidelines (Mohan, 2015):

i) Orthogonal Quality: ANSYS recommends the minimum orthogonal quality above 0.01 and the average to be much higher than this value. 
The minimum value from simulation was 0.46 and the average was near to 1.

ii) Jacobian: Jacobian is the measure of the deviation of an element from an ideally shaped element and should be greater than 0.511 . The simulation had minimum Jacobian ratio of 1 .

iii) Mesh Skewness: Skewness checks how close an element is to an equilateral triangle. The lower the skewness, the better the quality of mesh. The maximum skewness for accepting a mesh was taken as 0.94 for any mesh (Lee and Huang, 2014). The maximum skewness was 0.85 in the current simulation and the number of these highly skewed mesh were very low $(0.03 \%)$.

According to a study on nonlinear analysis using ANSYS by Bhashyam (2002), higher order elements are recommended in these simulations. The use of higher order tetrahedral underperformed compared to the higher order hexahedral in nonlinear elasto-plastic materials by the study of Benzley, Perry, Merkley, Clark (1995). The academic version of ANSYS has a limit on the number of nodes, which limited finer refinement on higher order elements. Hence, the analysis was performed with higher and lower order hexahedral and higher order tetrahedral and comparison was made for final selection.

Mesh selection consisted of comparing hexahedral with midnode (Solid186), tetrahedral with midnode (Solid187), and hexahedral without midnode (Solid185) by refining each type of mesh until the value converged below $10 \%$ and then comparing the results. All the meshes gave fairly accurate results within $2 \%$ on the values outside the contact of the shaft hub connection as verified by the DIN standard and experimental result. The value in the contact region varied from element to element and size of mesh.

The mesh from each element type was refined until the result converged to $10 \%$ difference in von Mises stress and then were compared inter-elemental. The meshes are shown in Figures 16, 17, and 18 for P4C shaft and hub connection. For P3G polygonal profile shaft and 
hub connection, the result from all three types of meshes were found close to each other with maximum difference of $7.23 \%$ between the meshes for maximum von Mises stress. However, for the P4C profiles, the result from the hexahedral and tetrahedral mesh showed similar distribution, but the difference in maximum von Mises stress was greater than $10 \%$. Due to the limitation of the academic version of the software, the maximum number of nodes that could be used was 256,000, which limited refinement of higher order hexahedral and tetrahedral mesh. The higher order meshes couldn't be refined adequately to converge the solution. The hexahedral mesh without mid node (SOLID185 with 8 nodes), could be refined to a contact element size of $0.35 \mathrm{~mm}$ and showed good convergence in P4C profiles and was used for all simulation purposes. Figures 18 and 19 show the selected mesh for P4C and P3G profiles respectively.

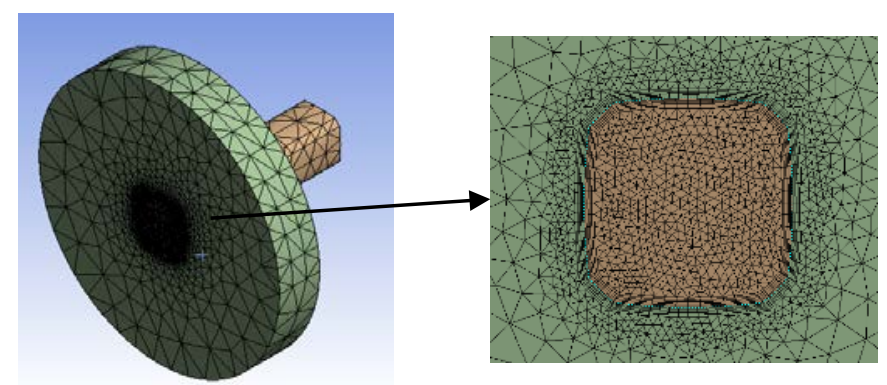

Figure 16: Tetrahedron Mesh with inflation in P4C connection

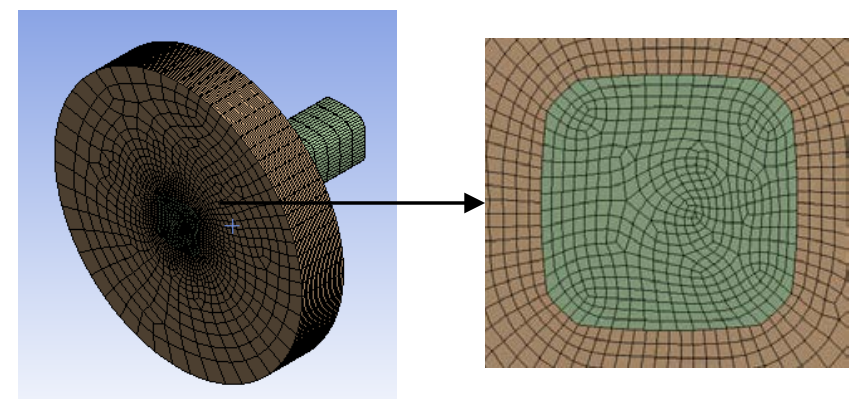

Figure 17: Hexahedral mesh with mid node in P4C connection 


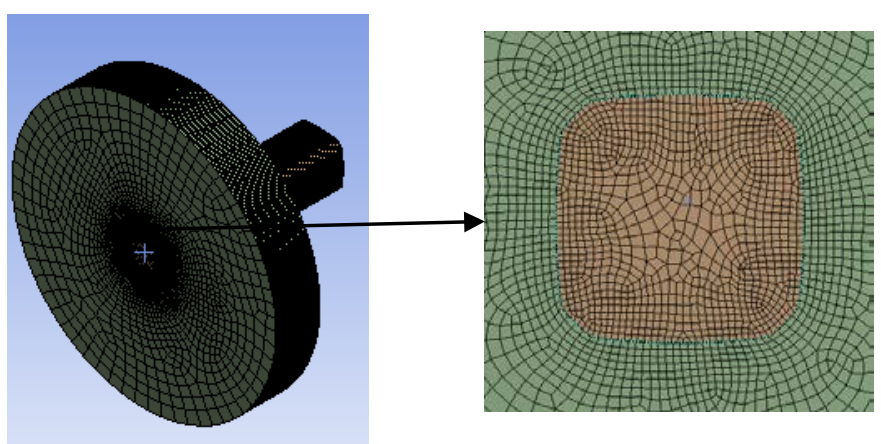

Figure 18: Hexahedral mesh without mid node in P4C connection (selected mesh)

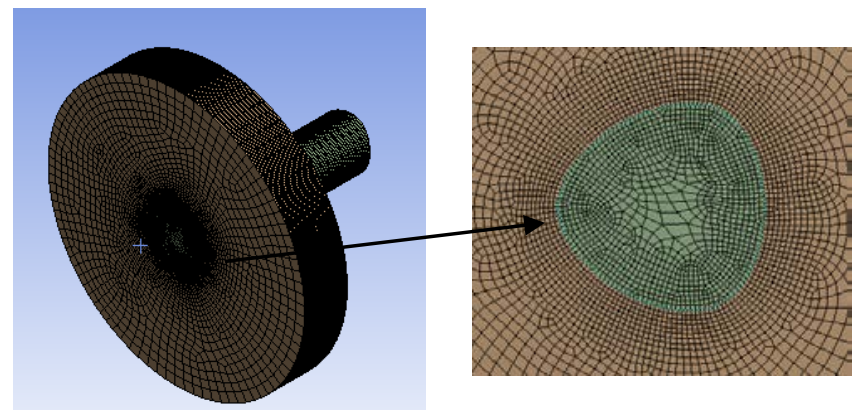

Figure 19: Hexahedral mesh without mid node in P3G connection (selected mesh)

3.4) Validation of results from FEM to experimental, analytical and DIN standard

After selecting the best mesh, a pilot study was performed to validate the results of FEA with the analytical, DIN standard and the experimental results using strain gage.

\section{5) Detailed Finite Element Analysis}

After verification of the finite element method, the comparisons were made between the two profiles for each fit and between each profile for various fits. 


\section{Chapter 4: Results/Discussion}

\section{1) Model Validation}

The FEA model was validated experimentally, analytically and from the DIN standard. This section explains the finite element model validation and has been divided into four sections, viz. modeling and convergence of FEM, validation from DIN standard, validation from experimental setup, and validation from analytical method. These sections are explained as follows.

\subsection{1) Modeling and Convergence of Nonlinear problems in FEA}

Selection of incorrect nonlinear settings or inappropriate elements could cause convergence at an incorrect value or may not converge at all. The effect of various factors on the convergence of the current nonlinear contact problem are explained as follows:

\section{i) Element type}

Although all the element types converged to a nonlinear solution, the results obtained were not consistent. The lower order hexahedral mesh could be refined to a contact size of 0.35 $\mathrm{mm}$, while the higher order elements could only be refined up to $0.5 \mathrm{~mm}$ for acceptable quality. This led to a better convergence result from hexahedral without mid node. This was evident from the result from the higher order hexahedral mesh that gave a different position of maximum stress in the limiting case of refining, while the lower order hexahedral gave the correct position for a similar number of elements. The restriction on the number of nodes hindered further refining in the higher order mesh. Also, the higher order tetrahedral mesh with inflation around the shaft and the hub connection, gave a much higher stress in the four lobe connection. The result is presented in Table 4, where the tetrahedral with inflation shows higher stress results than other meshes. The inflated zone has wedge 15 elements. This caused the error as the wedge 15 constituted most of the contact region and is recommended only for low stress gradient regions 
by ANSYS software. On the other hand, the tetrahedral mesh without the inflation zone and with local face refining gave similar results to the hexahedral mesh for a P3G connection. For the P4C connection, however, the result from this mesh did not match other meshes since the contact size could not be decreased below $0.62 \mathrm{~mm}$ in tetrahedral face mesh, although the result converged for all case.

Table 4: Performance of various mesh elements

\begin{tabular}{|c|c|c|c|c|}
\hline Type of Mesh & Profile & Fit & Load & $\begin{array}{r}\text { Maximum } \\
\text { von Mises } \\
\text { Stress (MPa) }\end{array}$ \\
\hline Tetrahedral mesh with local inflation & \multirow{4}{*}{ P3G } & \multirow{4}{*}{ Clearance } & \multirow{7}{*}{$\begin{array}{l}80 \mathrm{Nm} \\
\text { torque and } \\
4600 \mathrm{~N} \\
\text { radial load }\end{array}$} & 1013.0 \\
\hline Tetrahedral mesh with face refining & & & & 871.0 \\
\hline Hexahedral Mesh with mid node & & & & 856.0 \\
\hline Hexahedral Mesh with mid node & & & & 896.3 \\
\hline Tetrahedral mesh with local inflation & \multirow{3}{*}{$\mathrm{P} 4 \mathrm{C}$} & \multirow{3}{*}{ Clearance } & & 2142.1 \\
\hline Hexahedral Mesh without mid node & & & & 1326.4 \\
\hline Hexahedral Mesh with mid node & & & & 1388.9 \\
\hline
\end{tabular}

ii) Contact and Target Specification

The nonlinear contact between two materials is performed in ANSYS by defining one of the surfaces as contact and the other as target. For a convex surface coming in contact with a concave surface, the convention is to use concave surface, in this case the hub, as target (Benzley et al., 1995). For simulation, hub was considered as target. The solution was performed by taking symmetrical contact, which made the choice irrelevant. A sample simulation was performed to check the indifference of the contact and target specification that yielded the same result for either specification in symmetric behavior.

iii) Normal Stiffness

Two bodies in contact do not penetrate each other physically. To emulate the situation in simulation, ANSYS assumes a spring, which prevents the interpenetration between the two 
bodies, whose stiffness is defined under normal stiffness. Too much stiffness can increase accuracy, but may lead to divergence as well. Too small spring stiffness may lead to penetration and incorrect results. To find the optimal stiffness, the solution was initially performed at a normal stiffness of 0.001 . Once converged in lower stiffness, the solution was performed at higher stiffness value. The lower stiffness solutions were found to yield lower maximum von Mises stress. The lower stiffness allowed penetration, which did not increase the reaction force further and the resultant stress was lower. However, the result for stiffness of 0.1 and 1 were similar due to the effect of Lagrangian coefficient and the final simulation was performed at a normal stiffness of 1 .

\section{iv) Stabilization Damping Factor}

In the cases of clearance fit between the shaft and the hub, the ends of the shaft were fixed and the load was applied to the hub. The hub was not supported anywhere and suffered from rigid body motion when the force was applied. To avoid such rigid body motion, contact stabilization was added to the simulation. The contact stabilization introduced a viscous damping traction proportional, but opposite to the relative pseudo velocities between the shaft and the hub connection along the contact normal and tangential directions and thus avoided the rigid body motion (Higgins, 2012). The contact stabilization can be explained with the help of Figure 20. The use of damping coefficient of 5 and 0.2 did not seem to affect the solution. To avoid unnecessary damping, the solution was performed with a damping coefficient of 0.2.

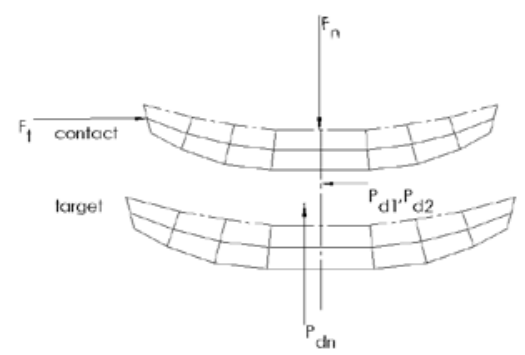

Figure 20: Schematic of contact stabilization damping factor 
where, $\mathrm{P}_{\mathrm{dn}}=\mathrm{d}_{\mathrm{n}} \dot{\mathrm{u}}_{\mathrm{n}}, \mathrm{P}_{\mathrm{d} 1}=\mathrm{d}_{\mathrm{t}} \dot{\mathrm{u}}_{1}, \mathrm{P}_{\mathrm{d} 2}=\mathrm{d}_{\mathrm{t}} \dot{\mathrm{u}}_{2}$

dn is the damping coefficient in the normal direction, $\mathrm{d}_{\mathrm{t}}$ is the damping coefficient in the damping coefficient in the tangential direction, ů is the pseudo velocity in respective direction.

v) Pinball Size

In the simulation with clearance fit, the contact between the parts was initially open, i.e. not touching each other. So, to specify the contact search area, a pinball region was declared in terms of the radius of a ball to help the software detect the contact zone. The pinball region showed the distance up to where the contact could find the target. The pinball size was changed to auto detection that allowed a pinball of slightly larger size than the gap or penetration between the parts. If the pinball size was smaller than gap of $0.058 \mathrm{~mm}$, the solution would not be achieved as the contact would not be detected.

vi) Contact Behavior

Symmetrical contact behavior was selected between the contacts. Symmetrical contact behavior helped to avoid the contact penetration on both target and contact and freedom to select contact and target surface, although it was computationally expensive.

vii)Contact Formulation

Augmented Lagrange was selected as the contact formulation.

viii) Contact Detection Method

Initially, the contact detection method was set at Gaussian points. This resulted in line-toline contact. The lower stress case had abnormal irregular spikes, which was resolved after changing the contact detection method to nodal projected normal from contacts as suggested in ANSYS INC., 2014. The contact pressures were found to be less spiky and had smoother transition using projected contact detection method. 
ix) Newton Raphson Option

The unsymmetrical Newton Raphson option was enabled because of friction which produces an unsymmetrical stiffness matrix. The difference between the use of full Newton Raphson method (ANSYS default) and the full unsymmetrical Newton Raphson method can be seen from Table 5, which shows no effect of the Newton Raphson option for frictionless case and significant effect in hub for frictional case.

Table 5: Table showing the effect of Newton Raphson option in solution

\begin{tabular}{|r|r|r|r|r|r|}
\hline Profile & Fit & Friction & $\begin{array}{c}\text { Newton Raphson } \\
\text { Option }\end{array}$ & $\begin{array}{l}\text { Maximum von } \\
\text { Mises stress in } \\
\text { Hub (MPa) }\end{array}$ & $\begin{array}{l}\text { Maximum von } \\
\text { Mises stress in } \\
\text { shaft (MPa) }\end{array}$ \\
\hline P4C & Transition & Yes & Default (Full) & 1027.90 & 629.73 \\
\hline P4C & Transition & Yes & Full Unsymmetrical & 888.59 & 617.17 \\
\hline P4C & Transition & No & Default (Full) & 1275.00 & 679.46 \\
\hline P4C & Transition & No & Full Unsymmetrical & 1275.00 & 679.46 \\
\hline
\end{tabular}

\section{x) Friction}

The effect of friction on the solution has been explained in detail later in this report and all the analyses were performed with frictional coefficient of 0.18 (Grossman, 2007).

\subsection{2) Validation from DIN standard}

To validate the FEA model from DIN standard, a torsional load of $80 \mathrm{Nm}$ was applied from the hub. The results from the FEA and the DIN standard are shown in Table 6 . The results from FEA is for a frictionless connection of line to line fit as in considered in DIN standard. From Table 6, the result of FEA seems to be very close to the theoretical maximum torsional shear stress, with percentage difference being only $3.52 \%$ and $1.88 \%$ for P3G and P4C shaft respectively. This shows that the result from the FEA can be relied to find the torsional shear stress developed in the shaft. 
Table 6: Comparison of FEA and DIN standard for torsional loading of $80 \mathrm{Nm}$

\begin{tabular}{|c|c|c|r|r|r|r|r|}
\hline \multirow{5}{*}{ Profile } & \multicolumn{2}{|c|}{$\begin{array}{c}\text { Maximum torsional shear } \\
\text { stress (MPa) }\end{array}$} & \multicolumn{4}{c|}{ Contact Stress (MPa) } \\
\cline { 2 - 7 } & FEA & DIN & $\begin{array}{c}\text { Percentage } \\
\text { Difference } \\
(\%)\end{array}$ & Maximum & $\begin{array}{c}\text { Average } \\
\text { excluding } \\
\text { zero stress } \\
\text { values }\end{array}$ & $\begin{array}{c}\text { Average } \\
\text { including } \\
\text { zero stress } \\
\text { values }\end{array}$ & DIN \\
\hline P3G & 154.95 & 160.50 & 3.52 & 2111.30 & 1147.72 & 407.04 & 226.51 \\
\hline P4C & 165.54 & 168.69 & 1.88 & 2938.00 & 800.53 & 142.32 & 128.82 \\
\hline
\end{tabular}

Polygonal shafts rarely fail from the static loads. One of the common failures is by fretting fatigue (Grossman, 2007). The shaft and hub connection edge act as a site of crack initiation and shafts fail by fracture and pitting. The contact stress developed at the shaft hub interface is the reason for the crack initiation. Contact stress is the major stress in the shaft hub connection and is highest at the edge of the shaft and hub connection (Grossman, 2007). Hence, validation of the contact stress values from the DIN standard are important for design. For the current analysis, the comparison has been made on the contact stress at the edge of shaft hub interface for a line to line fit as in the DIN standard. Even though it is a line to line fit, the hub expands as a result of the applied torque and forms distinct areas of contact instead of the whole region. The number of areas of contact is equal to the number of lobes in the profile.

For P4C shaft, FEA shows the maximum contact stress to be much larger compared to the contact stress from the DIN standard (22.81 times the contact stress value from the DIN standard). The DIN standard calculates the average contact stress over the whole section and is much smaller than the maximum stress from FEA. By taking the average of the contact stress values excluding zero (assuming no contact in positions where stress is zero), the average is still found to be still much higher than the DIN standard (6.21 times the contact stress value from the DIN standard). If the average of all the contact stress values along the edge of the shaft hub 
connection are considered (including stress magnitude of zero), the values is 1.1 time the DIN standard, with a difference of $9.96 \%$.

For P3G shaft, the results are similar to the P4C shaft. The maximum contact stress is 9.32 times that of the DIN standard. The average contact stress excluding zero stress values (assuming no contact in positions where stress is zero) is 5.07 times the DIN standard and the average contact stress including the whole closed edge (including stress magnitude of zero) is 1.8 times the DIN standard, with difference of $57 \%$. This deviation is similar to the results from Winterfeld (2001), who found larger contact stress compared to DIN standard.

The deviation of the average contact stress from the DIN standard is due to the theoretical area of contact used in DIN standard. In an actual loading, the hub expands as a result of the torsional load, even in the transition fit, and there are distinct area of contact rather than the whole area. The distinct smaller area will have to bear greater contact pressure, leading to the discrepancy. The reason for greater maximum contact stress in P4C shaft is due to the larger maximum pressure angle than the P3G shaft that led to smaller contact area. A careful looks at the cross section of the P4C shaft shows the sharper edge at the discontinuity of the epitrochoidal curve and the grinding circle. This sharper edge has the largest pressure angle and the shaft hub connection has smaller contact area and larger contact pressure. Due to the uncertainty in the contact region and average contact pressure, validation of FEA model from DIN standard has been based only on the torsional shear stress. The contact stress from the DIN standard cannot be relied due to lack of friction and fit consideration in DIN standard for practical purposes as well as depicted by Winterfeld (2001). 


\subsection{3) Validation from Experimental Determination of stress}

Strain gages were used to experimentally verify the FEA results for three lobe and four lobe polygonal shaft and hub connections. For the purpose of the experiment, a shaft and hub connection was subjected to a static torque and bending load as shown schematically in Figure 21. The sizes of the shaft and hub are shown in Appendix A. The von Mises stress developed in the shaft was evaluated using the strain gage at definite locations and the values were compared to the results from the finite element method.

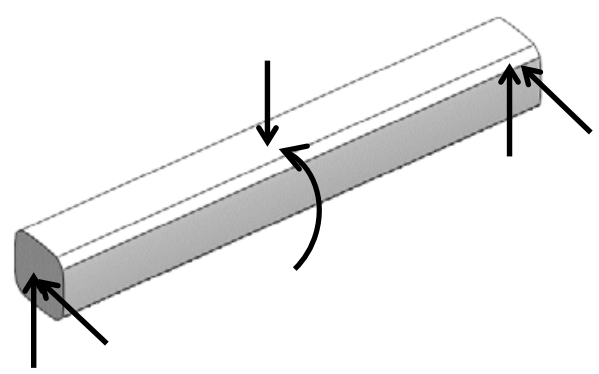

Figure 21: Loading for experimental setup

The calculation of von Mises stress is found in Appendix B. The experiment consisted of positioning the strain gage on the shaft, performing the experiment, and analyzing the results. The steps are briefly explained as follows:

i) Position of strain gage

The objective of the experiment was to verify the stresses in the shaft hub connection of FEA experimentally. Two stacked rectangular strain rosettes were used to measure strain at two positions for each polygonal shaft. The strain gage was placed in such a way that the middle strain gage was pointing in the axial direction as shown in Figure 22 and the gage was positioned in the middle of the face. The schematic of the strain gage placement is shown in Figures 23 and 24 for P3G and P4C connection respectively. 


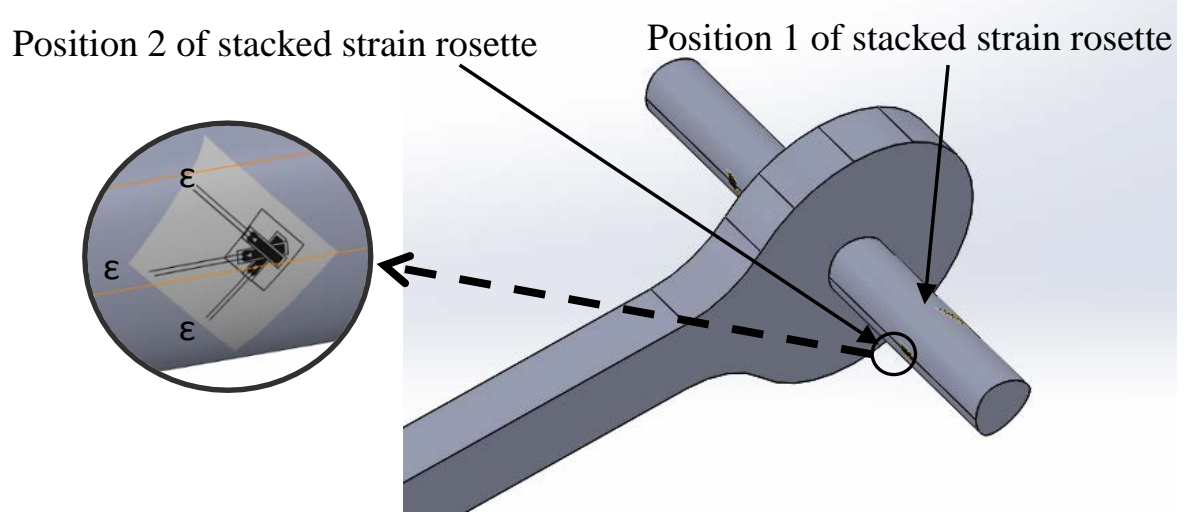

Figure 22: Position of strain gage in P3G

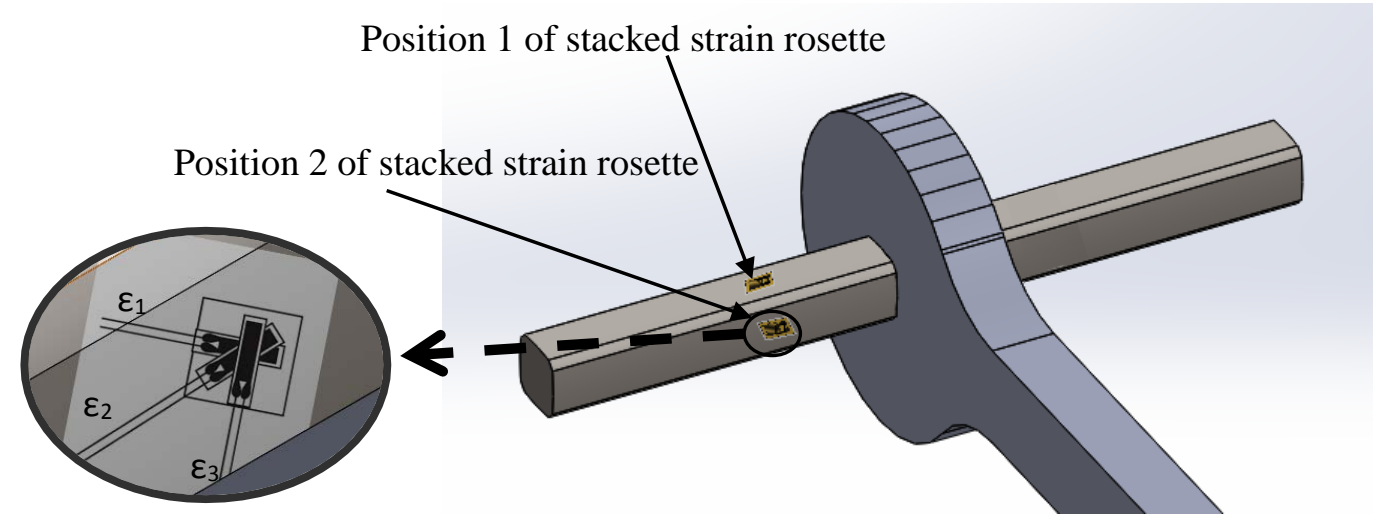

Figure 23: Position of strain gage in P4C shaft

\section{ii) Experimental Setup}

For the experimental setup, the hub was placed in the middle of the shaft. Although, the shaft was $152.4 \mathrm{~mm}$ long, only $101.6 \mathrm{~mm}$ was maintained between the two supports so as to avoid any slipping at the edge. The schematic of experimental setup is shown in Figure 24. The actual experiment is shown in Figure 25 for P4C connection and Figure 26 for P3G connection. A load of $6.82 \mathrm{~kg}(15 \mathrm{lb})$ was hung from the straight wrench with the help of chain of $0.794 \mathrm{~kg}$ so as to provide a torque of $22.13 \mathrm{Nm}$ and a radial load of $1012 \mathrm{~N}$ was applied from the top using an Instron universal testing machine (load cell precision $0.1 \%$ of load). The rosette strain gage and individual strain gage were composed of encapsulated $120 \Omega$ constantan metal foil with gage length of $0.787 \mathrm{~mm}$. 


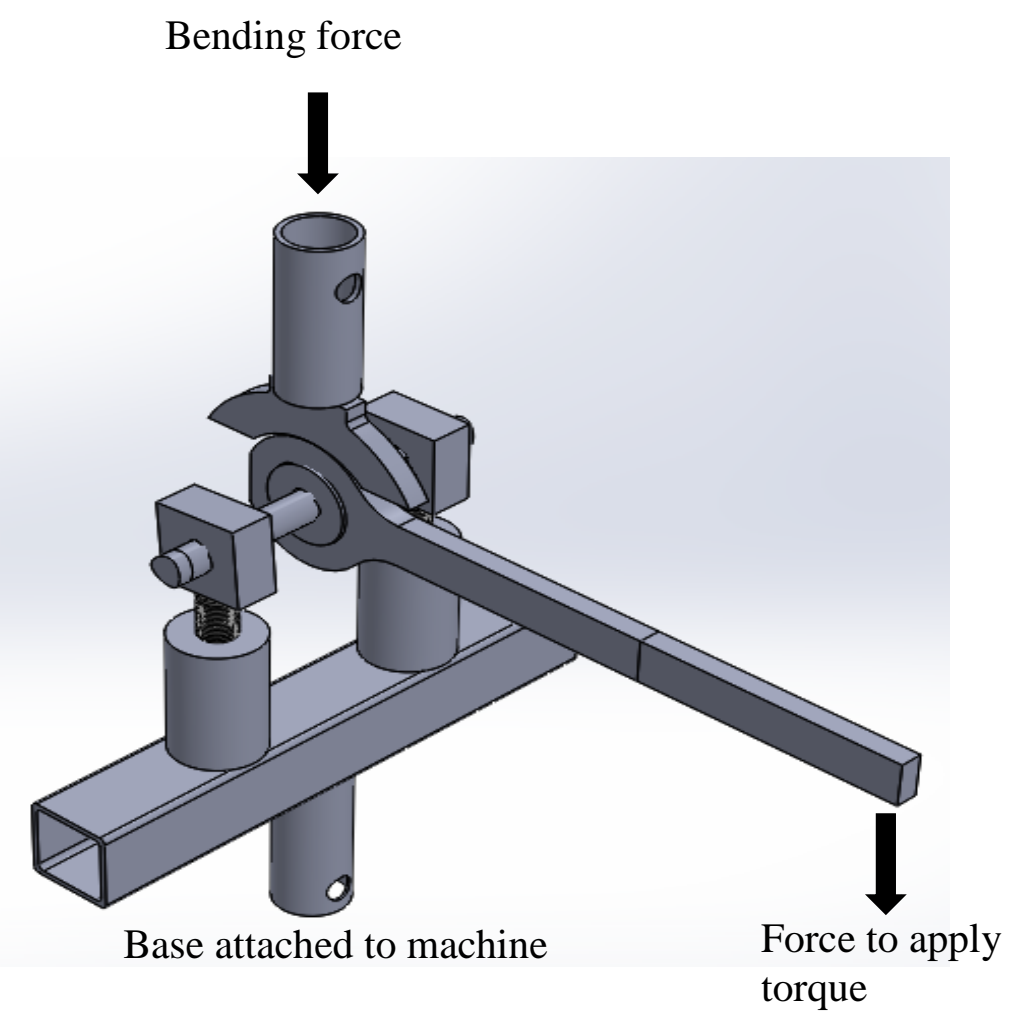

Figure 24: Schematic of experimental setup

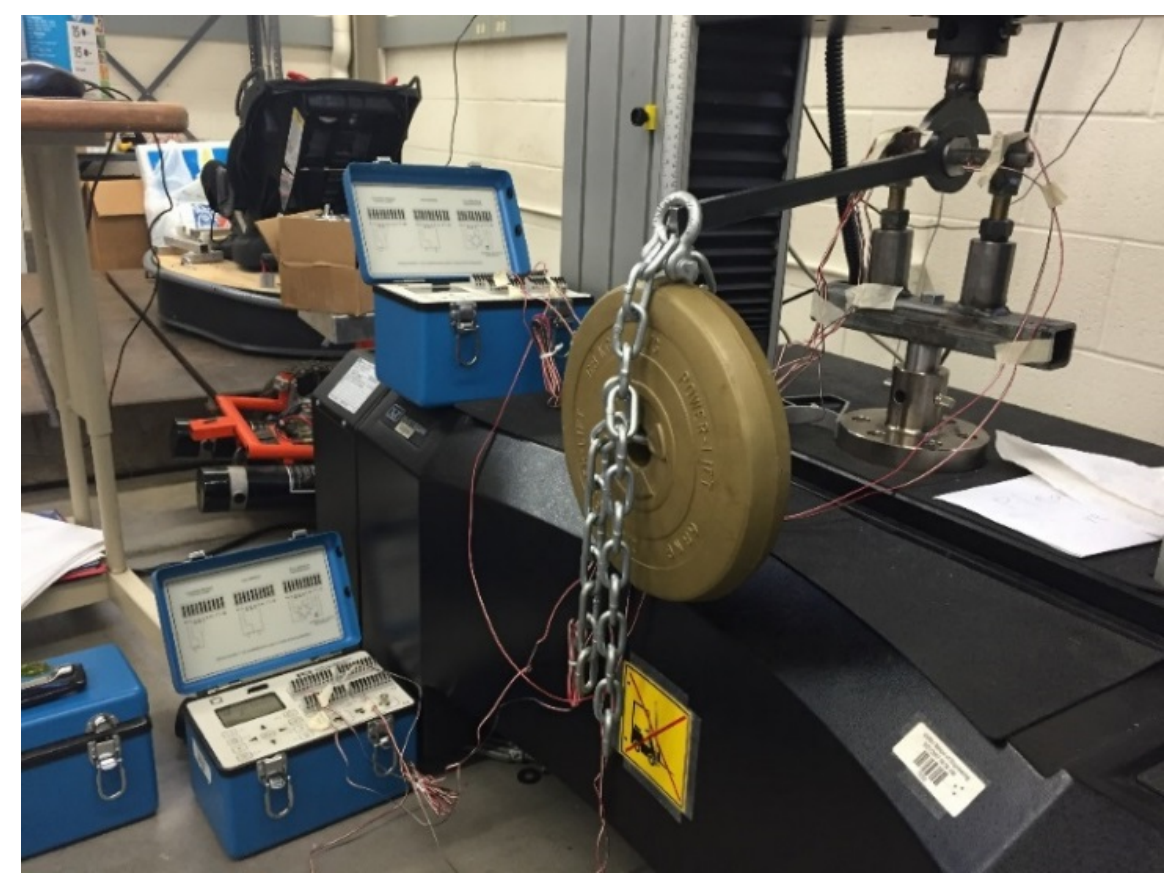

Figure 25: Experimental setup for strain measurement for P4C connection 


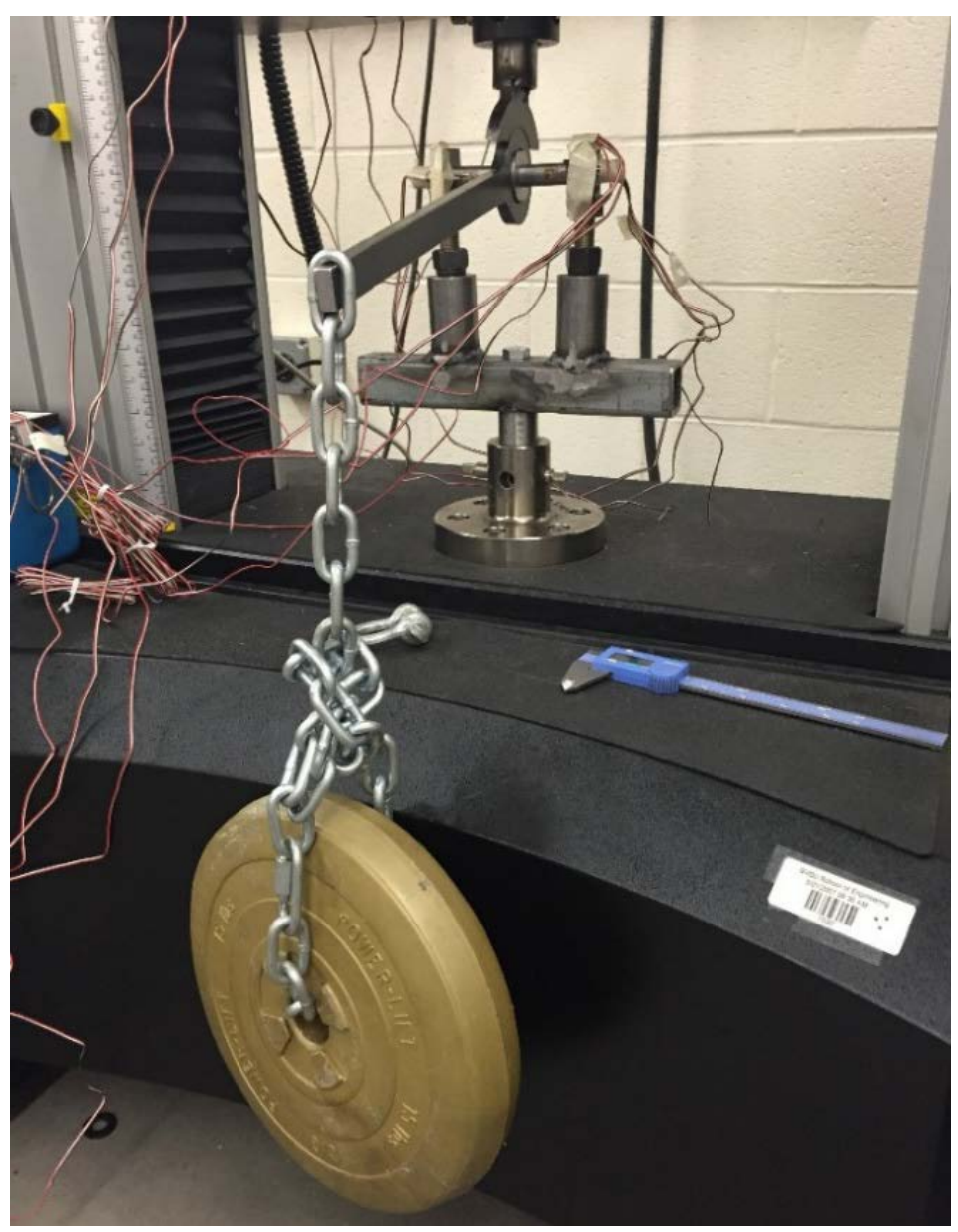

Figure 26: Experimental setup for strain measurement in P3G connection

For 4140 cold finished and annealed steel:

Young's Modulus of Elasticity $(\mathrm{E})=209$ GPa, Poisson’s ratio $(\mathrm{v})=0.3$, Modulus of rigidity $(\mathrm{G})=$ 80 GPa. Using strain recorder, the readings are shown in Table 7.

Table 7: Reading from the strain gage

\begin{tabular}{|c|c|r|r|}
\hline \multicolumn{1}{|c|}{ Strain Gage Type } & $\begin{array}{c}\text { Strain } \\
\text { Gage }\end{array}$ & $\begin{array}{c}\text { P3G } \\
\text { Value }(\boldsymbol{\mu \varepsilon})\end{array}$ & $\begin{array}{c}\text { P4C } \\
\text { Value }(\boldsymbol{\mu \varepsilon})\end{array}$ \\
\hline \multirow{3}{*}{ Stacked Strain Rosette 1 } & $\varepsilon_{1}$ & -107 & -135 \\
\cline { 2 - 4 } & $\varepsilon_{2}$ & -245 & 31 \\
\cline { 2 - 4 } & $\varepsilon_{3}$ & -71 & -150 \\
\hline \multirow{3}{*}{ Stacked Strain Rosette 2 } & $\varepsilon_{1}$ & 163 & -113 \\
\cline { 2 - 4 } & $\varepsilon_{2}$ & 17 & 52 \\
\cline { 2 - 5 } & $\varepsilon_{3}$ & -148 & 127 \\
\hline
\end{tabular}


The von Mises stress can be calculated from strain values in Table 7 using the equations in Appendix C and has been compared to the ones from the FEA result in Table 8.

Table 8: Table showing the comparison of FEA and experimental results

\begin{tabular}{|c|c|c|c|c|}
\hline \multirow{2}{*}{ Connection } & \multirow{2}{*}{ Gage } & \multicolumn{2}{|c|}{ von Mises stress (MPa) } & \multirow{2}{*}{ \% Error } \\
\hline & & FEA & Strain Gage & \\
\hline \multirow{2}{*}{ P3G } & Stacked Strain Gage 1 & 37.80 & 34.66 & 9.06 \\
\hline & Stacked Strain Gage 2 & 48.6 & 43.23 & 12.42 \\
\hline \multirow{2}{*}{$\mathrm{P} 4 \mathrm{C}$} & Stacked Strain Gage 1 & 42.08 & 44.41 & 5.25 \\
\hline & Stacked Strain Gage 2 & 34.97 & 37.22 & 6.05 \\
\hline
\end{tabular}

From Table, 8, the result from the FEA is validated since the percentage error to the results from the strain gage is less than $13 \%$ in all cases. The difference in result is due to the approximation in FEA and the difference in the ideal theoretical boundary condition used in FEA and the actual experimental one. 


\subsection{4) Validation from Analytical calculation of Shear Strength Distribution}

An analytical stress analysis of the polygonal shaft and hub is highly complex due to the conformal contact between the shaft and the hub and analysis has been concentrated on the shear stress only. Musyl calculated the stress and strain in a polygonal shaft subjected to a pure torsion load using small circles to approximate the profile (as cited in Grossman, 2007). This method has been followed by the DIN standard and provides the maximum shear stress. The standard, however, does not give the distribution of shear stress. This section approximates the shear stress distribution in the polygonal shaft subjected to pure torsion, using Saint Venant Torsion Theory and then compares the result to the FEA result for validation for three lobe and four lobe profiles.

Although, the shaft and hub application usually carry some bending load in addition to the major torsional loads, as in gears, the effect of such bending loads can be neglected in the case of small length shafts and higher torque applications to simplify the calculation. Hence, for these cases, it is sufficient to consider only the torsional loading on the shafts and determination of shear stress.

For applying Classical (Saint Venant's) Torsion theory to determine the shear stress distribution, the following assumptions were made, where $\mathrm{z}$ is along the axis of the shaft (Slivker, 2007):

i) Considering Z-axis to be along the axis, each cross-section (for each z) does not change its profile in the course of deformation. In other words, the bar's cross section behaves in its plane as if it were a rigid body rotating by a certain angle around the $\mathrm{z}$ axis.

ii) The rate of twist, $\mathrm{k}$, is a constant.

iii) Cross-sections are free to warp in the z-direction such that the points of every cross section can deviate from the original plane by the same amount. 
For a pure torsional load, there is no normal stress i.e. $\sigma_{\mathrm{x}}=0, \sigma_{\mathrm{y}}=0, \sigma_{\mathrm{z}}=0$ and there is no shear stress component along xy plane i.e. $\tau_{\mathrm{xy}}=0$. For a stress function, $\phi$, the boundary conditions are given by:

The outer contour of the shaft is stress free (away from the load location and end constraint) and the stress function is given as:

$$
\begin{aligned}
& \text { On the boundary, } \quad \begin{array}{l}
\quad \\
=
\end{array} \\
& \frac{\partial^{2} \phi}{\partial \mathrm{x}^{2}}+\frac{\partial^{2} \phi}{\partial \mathrm{y}^{2}}=-2 \mathrm{G} \theta
\end{aligned}
$$

where, $\mathrm{G}$ is the modulus of rigidity and $\theta$ is the angle of twist

Torque is given as:

$$
\mathrm{T}=-2 \iint \phi d y d x
$$

The shear stress can be expressed in terms of shear stress as:

$$
\begin{gathered}
\tau_{\mathrm{xz}}=-\frac{\mathrm{d} \phi}{\mathrm{dy}} \\
\tau_{\mathrm{yz}}=-\frac{\mathrm{d} \phi}{\mathrm{dx}}
\end{gathered}
$$

The stress function, $\phi$, should be defined in a way that equation (41) and (42) are satisfied. This value of $\phi$ can be differentiated in terms of $y$ and $x$, as shown in equations (44) and (45), to find the shear stresses.

The stress distribution has been compared with FEA for three and four lobe profiles as follows:

\subsubsection{1) FEA Validation from Shear Stress Distribution of P3G shaft}

For deriving the stress distribution using Saint Venant’s Principle, the same dimensions of the shaft were taken as used in experimental verification, i.e., a nominal diameter $\left(\mathrm{D}_{\mathrm{m}}\right)$ of $14.478 \mathrm{~mm}$ and eccentricity (e) of $0.508 \mathrm{~mm}$. The epitrochoidal equation could not be used for determining the stress function due to the complexity in derivation and was approximated by a 
similar profile. Taylor (1987) had derived an approximate shear stress distribution for hypocycloidal section. Similar hypocycloidal sections was used to approximate the shear stress distribution for a three lobe profile. The equation of the hypocycloidal curve used to approximate the given P3G profile is:

$$
\begin{array}{r}
\mathrm{x}=-\mathrm{a} \cos (v)-b \cos (2 v) \\
\mathrm{y}=\mathrm{a} \sin (v)-b \sin (2 v)
\end{array}
$$

The value of $a$ and $b$ needs to be determined that gives lowest variation between the actual curve and the approximation. Values of $a=7.201$ and $b=0.509$ gave the closest resemblance to the actual epitrochoidal curve and were determined by writing a MATLAB ${ }^{\circledR}$ program. These values are close to nominal radius and eccentricity of profile. The difference between the two plots is shown in Figure 27 and they are close to each other. The parameter $v$ starts from right side in the actual curve and from left side in this approximation.

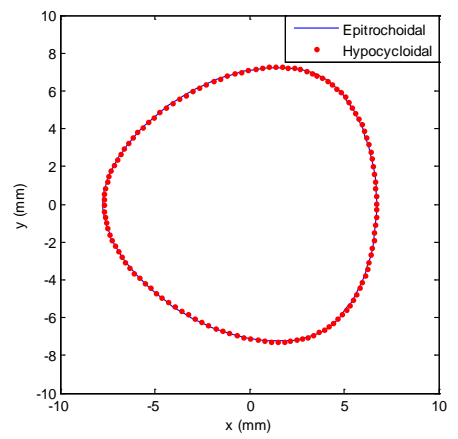

Figure 27: Comparison of actual (blue continuous) and approximation curve (red dotted) The loading of the shaft hub connection in FEA is shown in Figure 28.

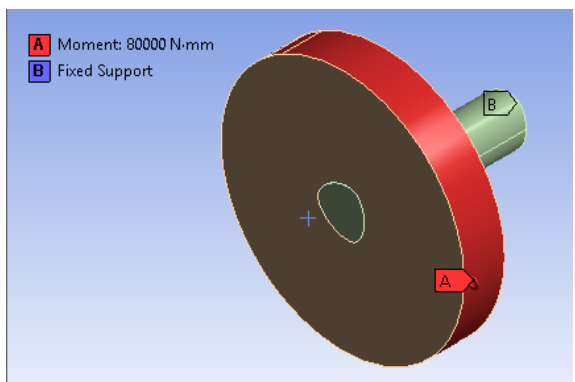

Figure 28: Torque application in a three lobe polygonal shaft FEA model 
For the torsional loading on a P3G polygonal shaft as shown in Figure 28, the torsional shear stress distribution was calculated using the Saint Venant Torsion principle as follows:

- $\phi=0$ on the boundary and $\frac{\partial^{2} \phi}{\partial \mathrm{x}^{2}}+\frac{\partial^{2} \phi}{\partial \mathrm{y}^{2}}=-2 \mathrm{G} \theta$

The stress function was considered in the same way as developed by Taylor (1987), which is obtained from equation (46) by eliminating the parameter $v$ and neglecting one term that did not satisfy equation (42) and can be written as:

$$
\phi=C\left(\left(a^{4}+a^{2} b^{2}-2 b^{4}\right)\left(x^{2}+y^{2}\right)-2 a^{2} b\left(-x^{3}+3 x y^{2}\right)-\left(a^{6}-3 a^{4} b^{2}+3 a^{2} b^{4}-b^{6}\right)\right)
$$

where, $\mathrm{C}$ is a constant

- Torque is given as:

$$
\mathrm{T}=-2 \int_{-7.747}^{6.731} \int_{\mathrm{y} 1}^{\mathrm{y} 2} \mathrm{C} \phi \mathrm{dydx}
$$

where,

$$
\begin{gathered}
y 1=\frac{\sqrt{\left(5.572 \times 10^{15} \mathrm{x}-9.507 \times 10^{16}\right)\left(1.857 \times 10^{15} \mathrm{x}^{3}-4.833 \times 10^{18}\right)}}{\left(5.572 \times 10^{15} \mathrm{x}-9.507 \times 10^{16}\right)} \\
y 2=-\frac{\sqrt{\left(5.572 \times 10^{15} \mathrm{x}-9.507 \times 10^{16}\right)\left(1.857 \times 10^{15} \mathrm{x}^{3}-4.833 \times 10^{18}\right)}}{\left(5.572 \times 10^{15} \mathrm{x}-9.507 \times 10^{16}\right)}
\end{gathered}
$$

Solving using trapezoidal method of numerical integration, $C=\frac{T}{2.214 \times 10^{7}}$

- The shear stress can be expressed in terms of shear function as:

$$
\begin{gathered}
\tau_{\mathrm{xz}}=-\frac{\partial \phi}{\partial \mathrm{y}} \\
\tau_{\mathrm{xz}}=\frac{\mathrm{T}(316.7 \mathrm{x}-5404) \mathrm{y}}{2.217 \times 10^{7}}
\end{gathered}
$$

Similarly,

$$
\begin{gathered}
\tau_{\mathrm{yz}}=\frac{\partial \phi}{\partial \mathrm{x}} \\
\tau_{\mathrm{yz}}=\frac{\mathrm{T}\left(158.4 \mathrm{x}^{2}+5404 \mathrm{x}-158.4 \mathrm{y}^{2}\right) \mathrm{y}}{2.217 \times 10^{7}}
\end{gathered}
$$


The comparison of FEA results and the analytical plot of the parametric equation for a torque of $80 \mathrm{Nm}$ is given by Figures 29, 30 and 31.

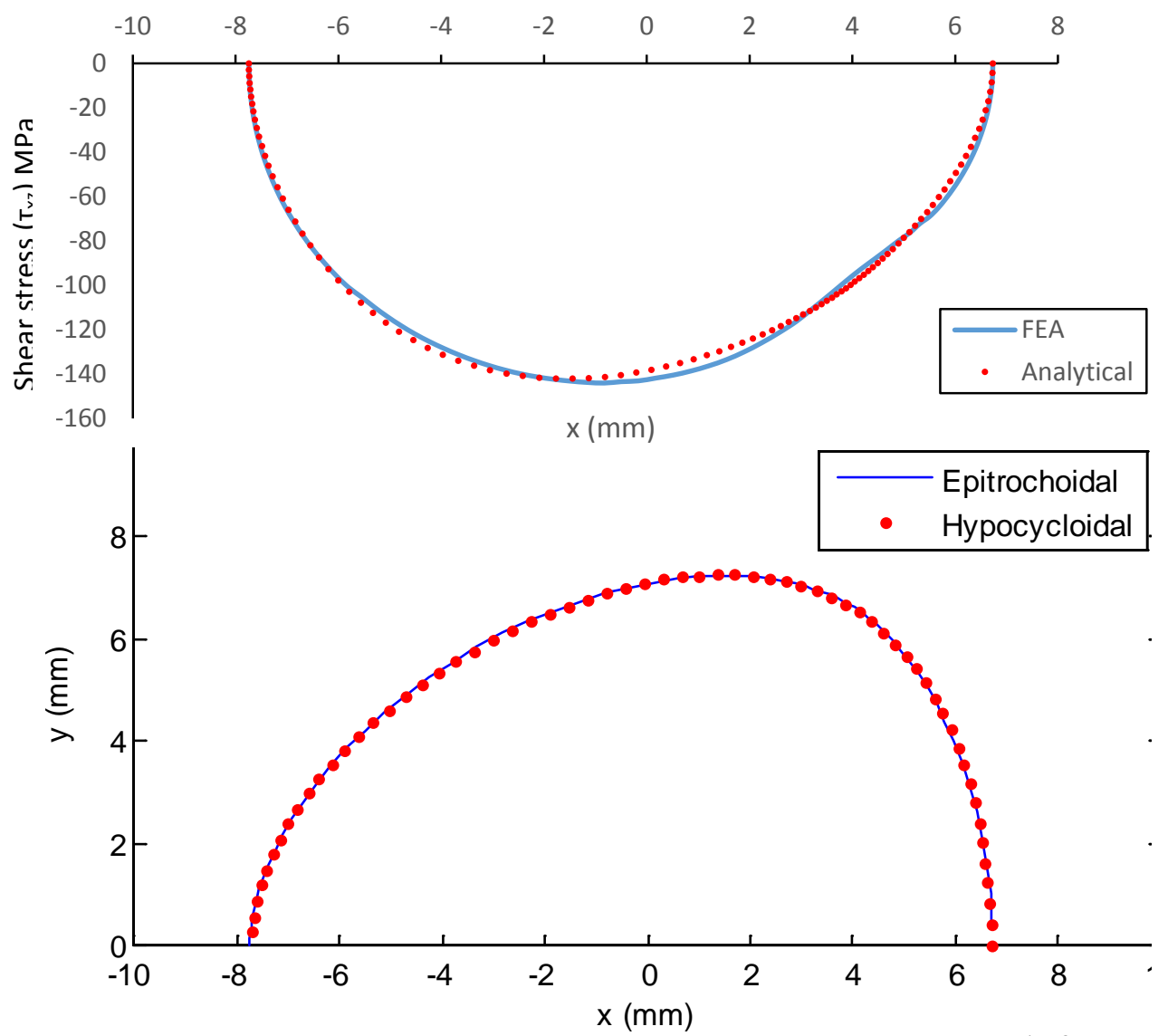

Figure 29: Comparison of FEA (blue solid) and Analytical (red dotted) result for $\tau_{\mathrm{xz}}$ of P3G shaft subjected to a torque of $80 \mathrm{Nm}$ (upper plot) with profile comparison (lower plot)

Figure 29 shows the distribution of shear stress in xz direction. The difference between the maximum stresses is $1.3 \%$ between the two methods. Figure 30 shows the distribution of shear stress in yz direction. The maximum stress value is $157.34 \mathrm{MPa}$ from analytical solution and 155 MPa from FEA, the minimum stress value is -116.92 MPa from analytical solution and -117 MPa from FEA, and the maximum difference is $1.5 \%$. 

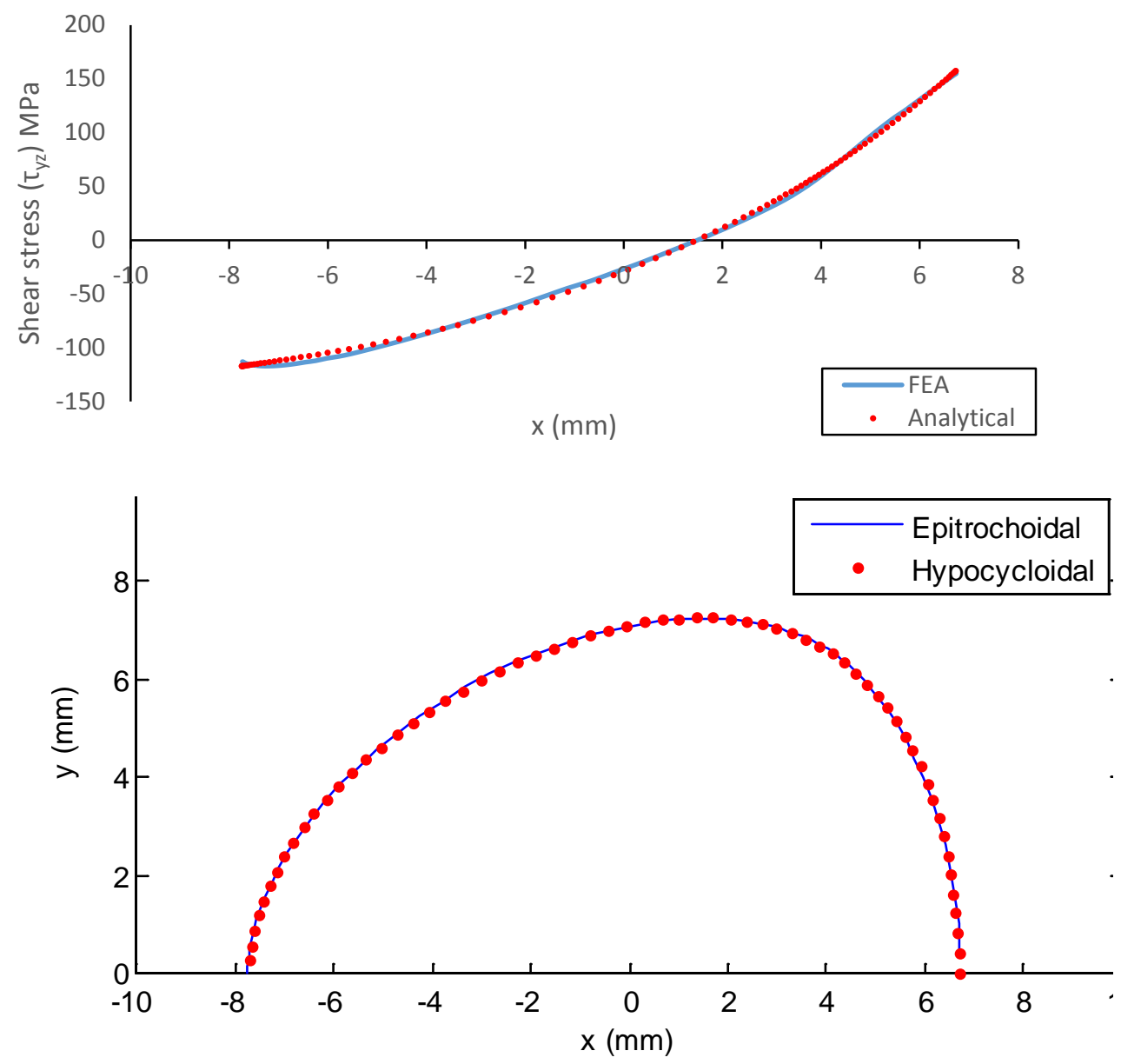

Figure 30: Comparison of FEA (blue solid) and Analytical (red dotted) result for $\tau_{\mathrm{yz}}$ of P3G shaft subjected to a torque of $80 \mathrm{Nm}$ (upper plot) with profile comparison (lower plot)

Figure 31 shows the distribution of maximum torsional shear stress. The maximum torsional shear stress has a difference of $0.88 \%$ between the two methods. The curves seem to match closely in the maximum and minimum shear stress regions and divert in between these values. 

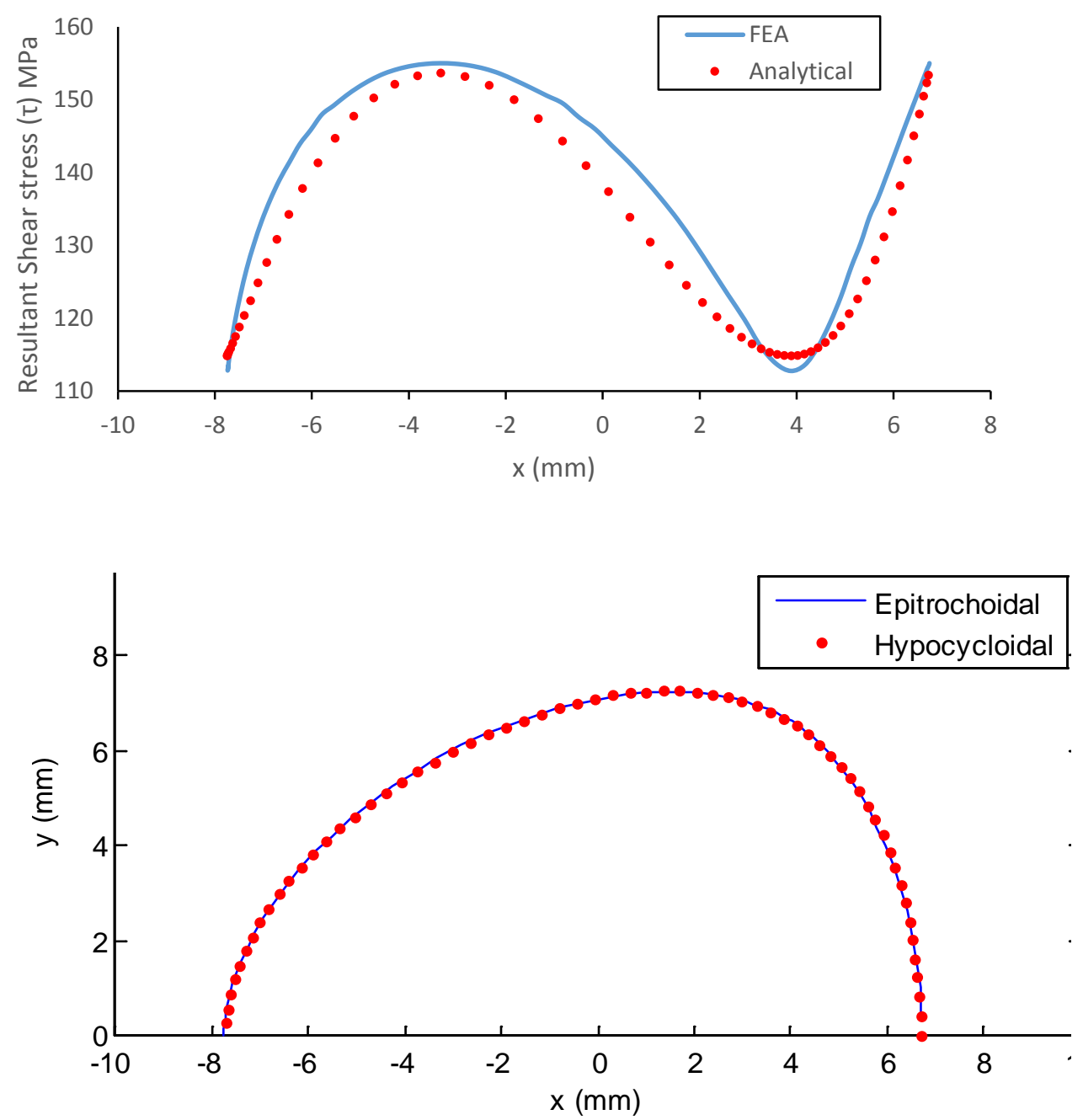

Figure 31: Comparison of FEA (blue solid) and Analytical (red dotted) result for torsional shear stress of P3G shaft subjected to a torque of $80 \mathrm{Nm}$ (upper plot) with profile comparison (lower plot)

The plots in Figure 29, 30, and 31 are very close to each other and the minor difference is due to the difference in epitrochoidal and hypocycloidal profile and the approximation in FEM and torsion principle. Hence, the FEA model is validated.

Based on such an accurate correlation of analytical and FEA, a general shear stress distribution for P3G shaft can be devised. For any P3G shaft of nominal diameter 2a and eccentricity b, the stress distribution is given as: 


$$
\tau_{\mathrm{xz}}=\mathrm{C}\left(2 \mathrm{y}\left(\mathrm{a}^{4}+\mathrm{a}^{2} \mathrm{~b}^{2}-2 \mathrm{~b}^{4}\right)-12 \mathrm{a}^{2} \mathrm{bxy}\right)
$$

Similarly,

$$
\tau_{y z}=C\left(2 x\left(a^{4}+a^{2} b^{2}-2 b^{4}\right)+2 a^{2} b\left(3 x^{2}-3 y^{2}\right)\right)
$$

where,

$$
C=\frac{\text { Torque }}{-2 \int_{-\mathrm{a}-\mathrm{b}}^{\mathrm{a}-\mathrm{b} 1} \int_{\mathrm{y} 1}^{\mathrm{y} 2} \phi \mathrm{dydx}}
$$

where,

$$
\begin{aligned}
& \phi=\left(\left(a^{4}+a^{2} b^{2}-2 b^{4}\right)\left(x^{2}+y^{2}\right)-2 a^{2} b\left(-x^{3}+3 x y^{2}\right)-\left(a^{6}-3 a^{4} b^{2}+3 a^{2} b^{4}-b^{6}\right)\right) \\
& y 2=\sqrt{\frac{\left(a^{4}+a^{2} b^{2}-2 b^{4}\right) x^{2}+2 a^{2} b x^{3}-\left(a^{6}-3 a^{4} b^{2}+3 a^{2} b^{4}-b^{6}\right)}{\left(a^{4}+a^{2} b^{2}-2 b^{4}-6 a^{2} b x\right)}} \\
& y 1=-y 2
\end{aligned}
$$

The integration needs to be solved using numerical integration.

\subsubsection{2) FEA Validation from Shear Stress Distribution of P4C shaft}

For finding the shear stress distribution in P4C profile, similar dimension were taken as the experimental verification, i.e., outer diameter $\left(d_{1}\right)$ of $15.875 \mathrm{~mm}$, inner diameter $\left(d_{2}\right)$ of $13.335 \mathrm{~mm}$, and eccentricity (e) of $1.905 \mathrm{~mm}$.

The parametric equation could not be used for determining the stress function. Hence, the parametric curve was approximated by piecewise polynomial. For the given four lobe polygonal curve, four polynomials were derived, one on each side, using Lagrange interpolation. The equations of the polynomial were:

$$
\left.\begin{array}{l}
x= \pm\left(-0.01378 y^{2}+6.651\right) \\
y= \pm\left(-0.01378 x^{2}+6.651\right)
\end{array}\right\}
$$


The comparison of the actual curve and the polynomial approximation is shown in Figure 32.

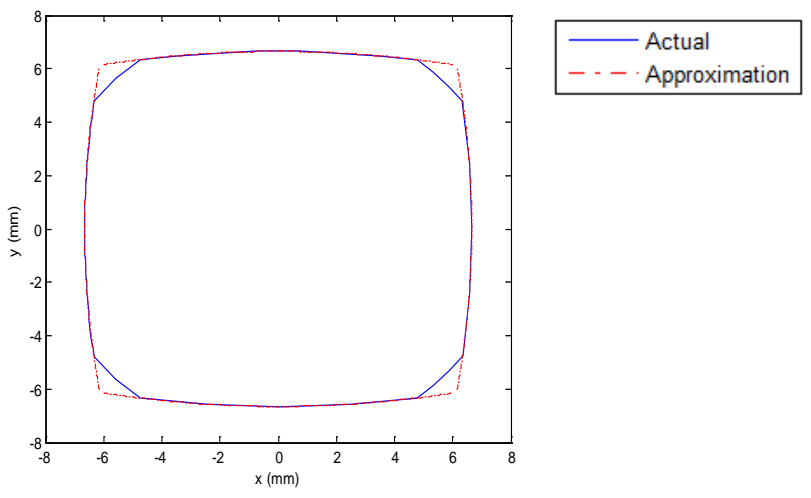

Figure 32: Comparison of actual (blue) and approximation curve (red discontinuous)

Referring to Figure 32, the two curves are close to each other in the middle portion, but much different at the corners. This means that the curve will not show correct value at the corners. The loading of the profile is as shown in Figure 33 for FEA.

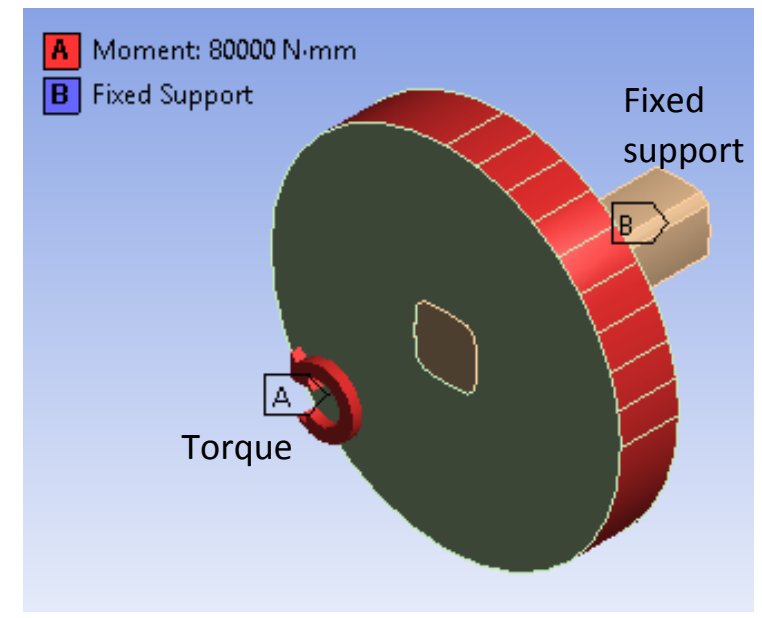

Figure 33: Torque application in a four lobe polygonal shaft FEA model

For a torsional loading on a P4C polygonal shaft, as shown in Figure 33, the torsional shear stress distribution was calculated using the Saint Venant's Torsion principle as follows:

- The stress function satisfying equation (41) and (42) from equation (52) is given as:

$$
\phi=\mathrm{C}\left(\mathrm{x}^{2}-\left(-0.01378 \mathrm{y}^{2}+6.651\right)^{2}\right)\left(\mathrm{y}^{2}-\left(-0.01378 \mathrm{x}^{2}+6.651\right)^{2}\right)
$$

where, $\mathrm{C}$ is a constant 
- Torque is given as (the portion of the circle has been neglected in torque calculation):

$$
\begin{gathered}
\mathrm{T}=-2 \iint \phi \mathrm{dyd} x \\
\mathrm{~T}=-2 \int_{6.34}^{6.651} \int_{\mathrm{y} 1}^{\mathrm{y} 2} \phi \mathrm{dydx}-2 \times 2 \int_{0}^{4.7} \int_{\mathrm{y} 3}^{\mathrm{y} 4} \phi \mathrm{dydx}
\end{gathered}
$$

where, $y 2=\sqrt{\frac{6.651-x}{0.01378}} ; y 1=-\sqrt{\frac{6.651-x}{0.01378}}$

$\mathrm{y} 3=-\left(-0.01378 \mathrm{x}^{2}+6.651\right)$

$\mathrm{y} 4=\left(-0.01378 \mathrm{x}^{2}+6.651\right)$

Solving, $C=\frac{T}{-251090.33}$

- The shear stress can be expressed in terms of shear function as:

$$
\begin{aligned}
& \qquad \tau_{\mathrm{xz}}=-\frac{\partial \phi}{\partial y} \\
& \tau_{\mathrm{xz}}=-2 \mathrm{Cy}\left(\mathrm{x}^{2}-\left(0.01378 \mathrm{y}^{2}-6.651\right)^{2}\right)-0.05514 \mathrm{Cy}\left(\left(-0.01378 \mathrm{x}^{2}+6.651\right)^{2}-\mathrm{y}^{2}\right)\left(0.01378 \mathrm{y}^{2}-6.651\right) \\
& \text { Similarly, } \tau_{\mathrm{yz}}=\frac{\partial \phi}{\partial \mathrm{x}} \\
& \tau_{\mathrm{xz}}=2 \mathrm{C}\left(\mathrm{x}^{2}-\left(0.01378 \mathrm{y}^{2}-6.651\right)^{2}\right)\left(0.02754 \mathrm{x}-6.485 \times 10^{-5}\right)\left(-0.01378 \mathrm{x}^{2} 6.651\right)-2 \mathrm{Cx}\left(\left(-0.01378 \mathrm{x}^{2}+6.651\right)^{2}-\mathrm{y}^{2}\right)
\end{aligned}
$$

The comparison between the FEA results and the analytical plot for a torque of $80 \mathrm{Nm}$ is given by Figures 34 and 35.

Figure 34 shows the distribution of shear stress in the $x z$ direction. The value of the maximum stress is $154.65 \mathrm{MPa}$ from the analytical solution and $165 \mathrm{MPa}$ from the FEA and the difference of $6.47 \%$. The analytical result values are slightly smaller than the FEA result. The irregularity in the plots occurs at the discontinuity of the curve. For the analytical result, since such discontinuity does not exist, as shown by red dotted plot in the lower plots, the irregularities are not present. Small irregularity is present at the end coordinates where the two curves meet. 

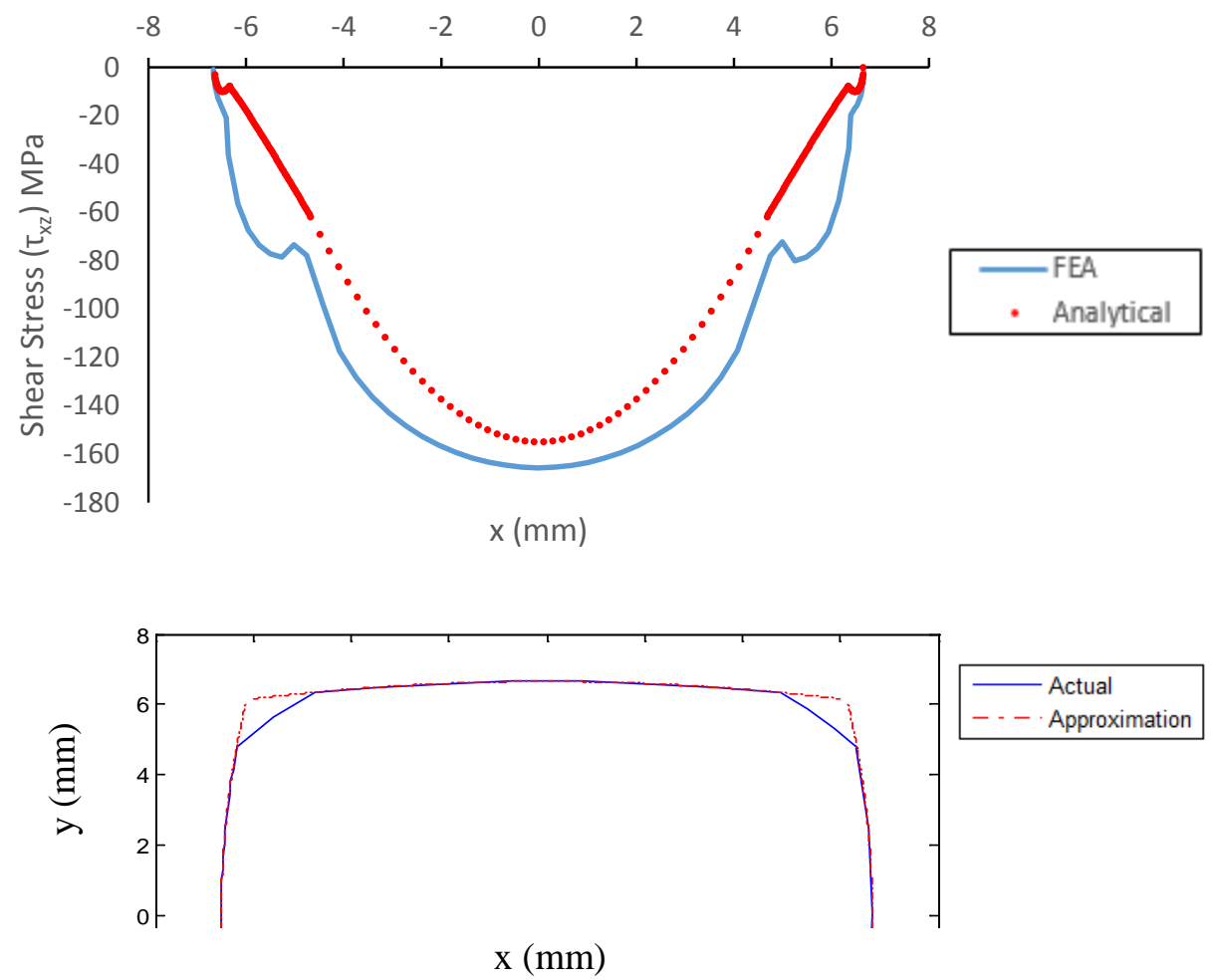

Figure 34: Comparison of FEA (blue solid) and Analytical (red dotted) result for $\tau_{\mathrm{xz}}$ of four lobe polygonal shaft subjected to a torque of $80 \mathrm{Nm}$ (upper plot) with profile comparison (lower plot)
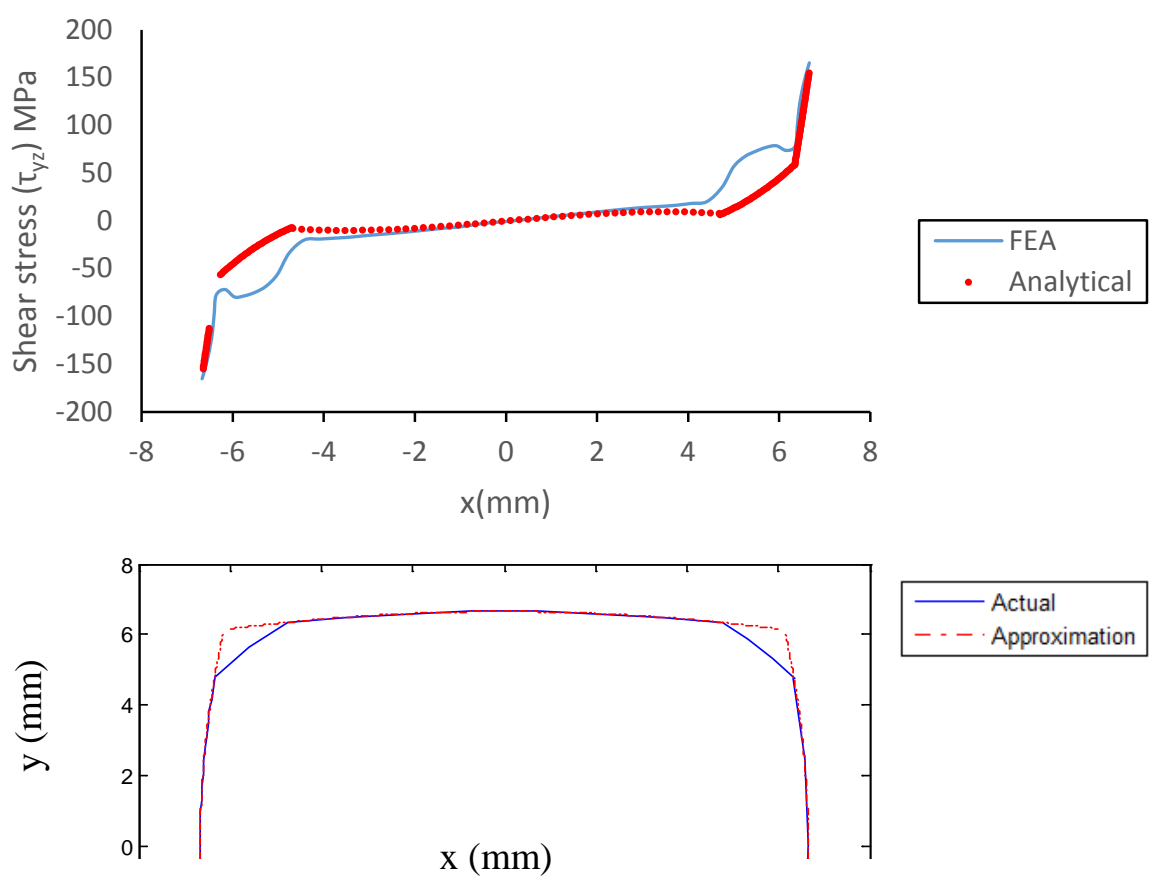

Figure 35: Comparison of FEA (red dotted) and Analytical (blue solid) result for $\tau_{\mathrm{yz}}$ of P4C shaft subjected to a torque of $80 \mathrm{Nm}$ (upper plot) with profile comparison (lower plot) 
The shear stress distribution in yz direction, shown in Figure 35, shows close conformance of the analytical distribution to the FEA solution. The only portion where differences occur is the circular portion at the corner, as shown in the bottom plot of Figure 35. The maximum stress is found to be $165.63 \mathrm{MPa}$ from FEA and 154.72 MPa from analytical solution and has a difference of $6.81 \%$.

Figure 36 shows the distribution in torsional shear stress and the difference between the maximum values is $6.8 \%$. The reason for the difference is the use of approximate curve in analytical method and neglecting shear stress in xy direction in the analytical method and approximation used in FEA. Overall, the FEA result is validated.
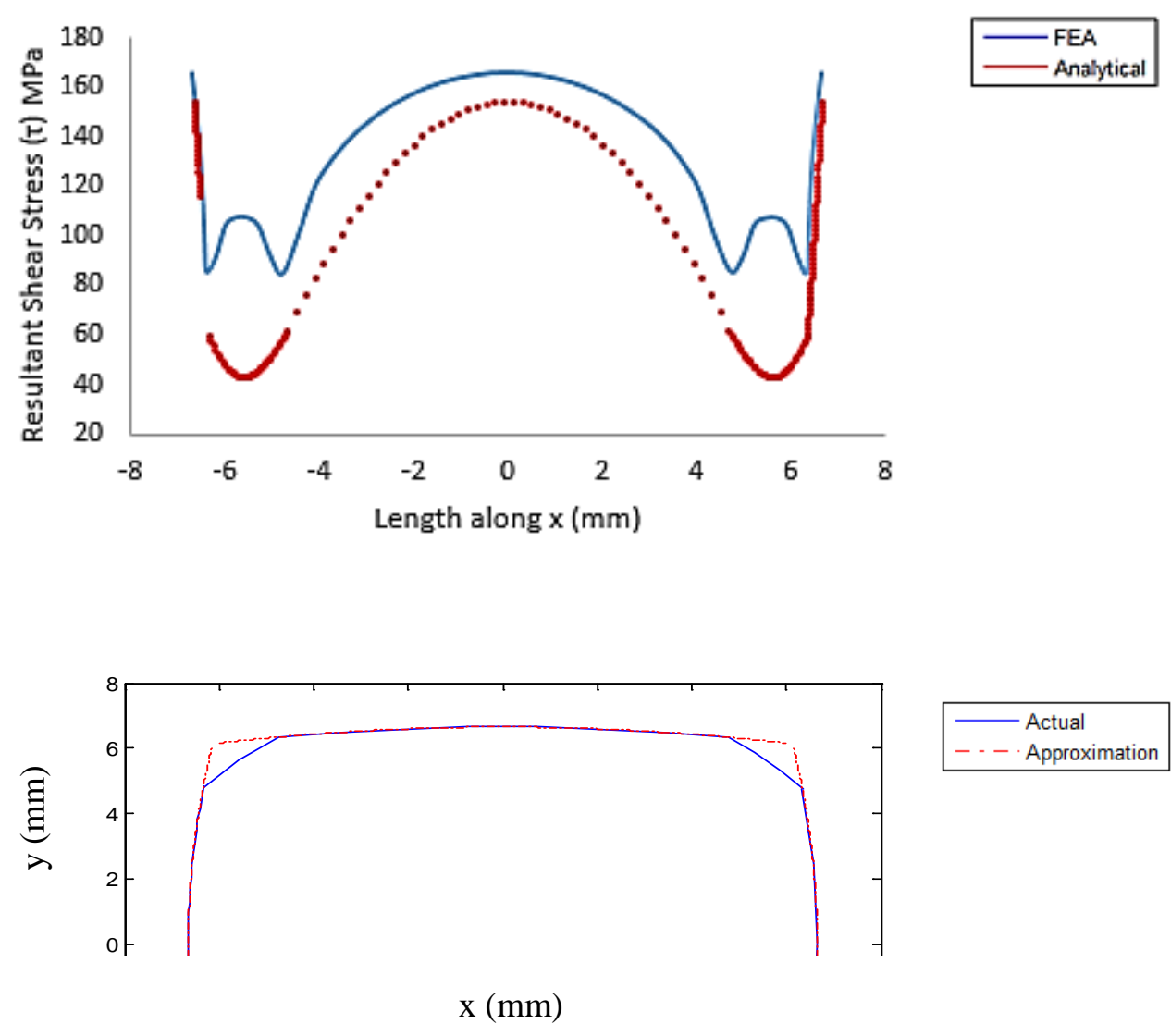

Figure 36: Comparison of FEA (red dotted) and Analytical (blue solid) result for resultant shear stress of four lobe polygonal shaft subjected to a torque of $80 \mathrm{Nm}$ (upper plot) with profile comparison (lower plot) 


\subsection{5) Validation from Analytical determination of point of contact}

The analytical calculation of point of contact for a shaft hub connection can be used to validate the FEA solution. The point of contact is also useful in finding the location of crack initiation for fatigue failure.

The location of the contact node from FEA and point of contact from analytical method has been compared for a clearance fit. The result has been presented by first determining point of contact for P3G shaft and then for P4C shaft and then comparing to FEA as follows.

4.1.5.1) Analytical Derivation of point of contact for P3G clearance fit using the method of Du Yanxia (2012).

The P3G clearance fit has:

Hub Nominal Diameter $\left(\mathrm{D}_{1}\right)=14.503 \mathrm{~mm}$

Shaft Nominal Diameter $\left(\mathrm{D}_{2}\right)=14.445 \mathrm{~mm}$

Eccentricity for hub $\left(\mathrm{e}_{1}\right)=0.508 \mathrm{~mm}$

Eccentricity for shaft $\left(\mathrm{e}_{2}\right)=0.508 \mathrm{~mm}$

Number of lobes $(n)=3$

Diametrical Clearance $\left(C_{d}\right)=D_{2}-D_{1}=-0.058 m m$

Value of parameter $v$ at the point of contact is given by equation (35)

For the hub:

$$
v_{1}=0.514
$$

Pressure angle from equation (20):

$$
\beta_{1}=0.207
$$

Angle from the center of the hub:

$$
\begin{gathered}
\phi_{1}=\beta_{1}+v_{1}=0.721 \\
\phi_{1}=\beta_{1}+v_{1}=41.29^{\circ}
\end{gathered}
$$

For the shaft using equation (36) as:

$$
v_{2}=0.533
$$


Pressure angle from equation (20):

$$
\beta_{2}=0.208
$$

Angle from the center of the hub:

$$
\begin{gathered}
\phi_{2}=\beta_{2}+v_{2}=0.741 \\
\phi_{2}=\beta_{2}+v_{2}=42.44^{\circ}
\end{gathered}
$$

4.1.5.2) Analytical Derivation of point of contact for P4C clearance fit.

The P4C clearance fit has:

Hub Nominal Diameter $\left(\mathrm{D}_{1}\right)=17.171 \mathrm{~mm}$

Shaft Nominal Diameter $\left(\mathrm{D}_{2}\right)=17.112 \mathrm{~mm}$

Eccentricity for hub $\left(\mathrm{e}_{1}\right)=1.905 \mathrm{~mm}$

Eccentricity for shaft $\left(\mathrm{e}_{2}\right)=1.905 \mathrm{~mm}$

Number of lobes $(n)=4$

Diametrical Clearance $\left(C_{d}\right)=D_{2}-D_{1}=-0.058 m m$

Solving for the point of contact as in P4C shaft using similar method to P3G shaft ended up with a value of $v$ comes near $22.5^{\circ}$, which does not exist in P4C profile. This occurred since the P4C polygonal shaft is not a harmonic curve. Hence, an approximate solution was used to approximate the contact location.

The point of contact will be in the epitrochoidal curve and not on the circular portion, so the solution can be approximated by solving the point of contact equation numerically near that region for a acceptable tolerance. The steps followed for finding the point of contact are explained as follows.

Solving the parametric equation and the equation of circle to find the point of contact. The equation of circle is:

$$
x^{2}+y^{2}=7.921^{2}
$$

The equation of the epitrochoidal curve is 


$$
\begin{gathered}
x=\left(\frac{17.112}{2}-1.905 \cos (4 v)\right) \cos (v)-7.62 \sin (4 v) \sin (v) \\
y=\left(\frac{17.112}{2}-1.905 \cos (4 v)\right) \sin (v)+7.62 \sin (4 v) \cos (v)
\end{gathered}
$$

where, $7.921 \mathrm{~mm}$ is the radius of outer diameter, $17.112 \mathrm{~mm}$ is the nominal diameter and 1.905 $\mathrm{mm}$ is the eccentricity.

From equations (55), (56), and (57)

$$
\left(\left(\frac{17.112}{2}-1.905 \cos (4 v)\right) \cos (v)-7.62 \sin (4 v) \sin (v)\right)^{2}+\left(\left(\frac{17.112}{2}-1.905 \cos (4 v)\right) \sin (v)+7.62 \sin (4 v) \cos (v)\right)^{2}=7.921^{2}
$$

Solving, $v_{2}=0.133$ is the point where the two curves meet. The corresponding value on the shaft is given by equation (35) as:

$$
v_{1}=\frac{\cos ^{-1}\left(\frac{e_{2} \cos \left(4 v_{2}\right)-\frac{C_{d}}{2}}{e_{1}}\right)}{4}
$$

At the point of contact, the radius of curvature of the hub and the shaft and the pressure angle should be the same. Using the range of $v_{2}$ and $v_{1}$ from 0.133 to 0.1 and finding the optimum value of parameters that will minimize the sum of absolute magnitude of difference in the radius of curvature and pressure angle, the true value can be determined. A MATLAB program was developed to find the optimum value.

Solving, $v_{1}=0.125$ and $v_{2}=0.133$.

For the hub,

Pressure angle from equation (20):

$$
\beta_{1}=0.486
$$

Angle from the center of the hub:

For the shaft:

$$
\begin{aligned}
& \phi_{1}=\beta_{1}+\mathrm{v}_{1}=0.611 \\
& \phi_{1}=\beta_{1}+\mathrm{v}_{1}=35.01^{\circ}
\end{aligned}
$$


Pressure angle from equation (20):

$$
\beta_{2}=0.509
$$

Angle from the center of the shaft:

$$
\phi_{2}=\beta_{2}+v_{2}=0.642=36.81^{\circ}
$$

The point of contact can be determined from the FEA by finding the coordinates of the node with the maximum value of contact pressure for pure torsion. The result is shown in Table 9.

Table 9: Comparison of analytical contact point determination to the FEA result.

\begin{tabular}{|r|r|r|r|}
\hline Profile & Method & \multicolumn{2}{|c|}{ Angle of Contact } \\
\cline { 3 - 4 } & & Hub & Shaft \\
\hline \multirow{2}{*}{ P3G } & FEM $\left(^{\circ}\right)$ & 41.15 & 42.03 \\
\cline { 2 - 4 } & Analytical $\left(^{\circ}\right)$ & 41.29 & 42.44 \\
\cline { 2 - 4 } & Difference $(\%)$ & 0.34 & 0.97 \\
\hline \multirow{2}{*}{ P4C } & FEM $\left(^{\circ}\right)$ & 34.81 & 34.65 \\
\cline { 2 - 4 } & Analytical $\left(^{\circ}\right)$ & 35.02 & 36.81 \\
\cline { 2 - 4 } & Difference $(\%)$ & 0.60 & 6.05 \\
\hline
\end{tabular}

From Table 9, it can be observed that the point of contact are close to each other from the two methods. The values are even closer in the P3G connection than the P4C connection. The difference in results is anticipated, since the analytical solution shows the geometric point of contact and FEA calculates the point after frictional sliding and deformation and varies slightly from the initial point of contact. This close conformance validates the FEA solution.

After validation of the model, the selected mesh was used for detailed finite element analysis. The results from the finite element analysis has been analyzed in two sections. The first section describes the result of the transient loading and the second section compares in detail the result from torsional bending and torsional loading on the two profiles. 


\section{2) Transient Loading}

A static torsional loading of $80 \mathrm{Nm}$ and rotating bending force of $4600 \mathrm{~N}$ were applied as shown in Figures 14 and 15 for transient loading that cover one period of the profile. The result of such loading for P3G transition fit and P4C clearance fit has been briefly described as follows:

\subsection{1) Transient Loading for P3G transition fit connection}

The load step for P3G polygonal profile for transition fit is shown in the upper plot of Figure 37, where the first load step is a ramped pure torsional load of $80 \mathrm{Nm}$ (shown by the blue line), which is then kept constant for rest of the load step. From the second load step, a constant magnitude bending load is applied varying in position along the circumference of the hub. The load position is radially varied at $10^{\circ}$ and covers the full $120^{\circ}$ range for the P3G profile. The convergence can be seen in the lower plot of Figure 37. The line search parameter and maximum DOF increment plot can be seen from Figure 38. The higher value of these parameters would mean rigid body motion. The lower DOF increment shows that there was not much displacement except the first load step where the initial contact occurred. Owing to low values, there was no rigid body motion
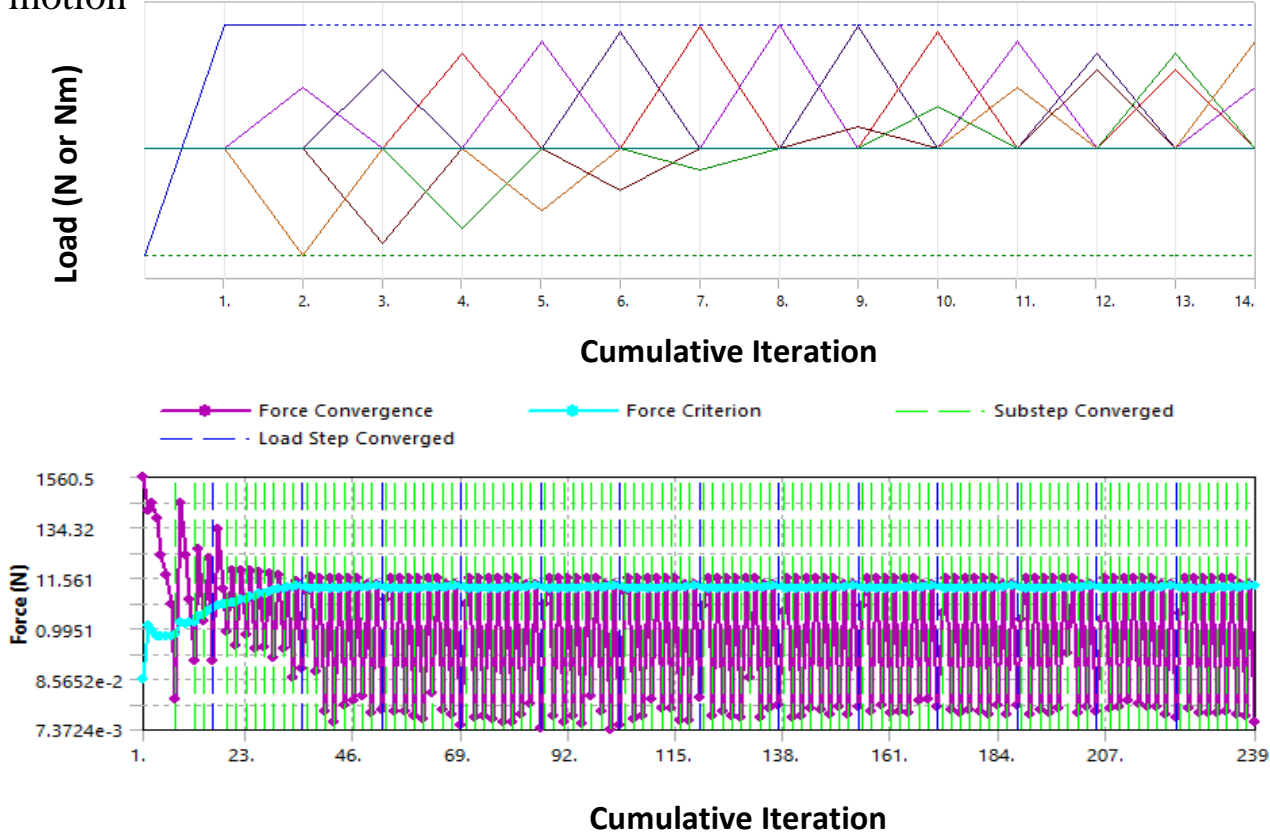

Figure 37: Load step (upper) and convergence (lower) for line fit P3G connection 

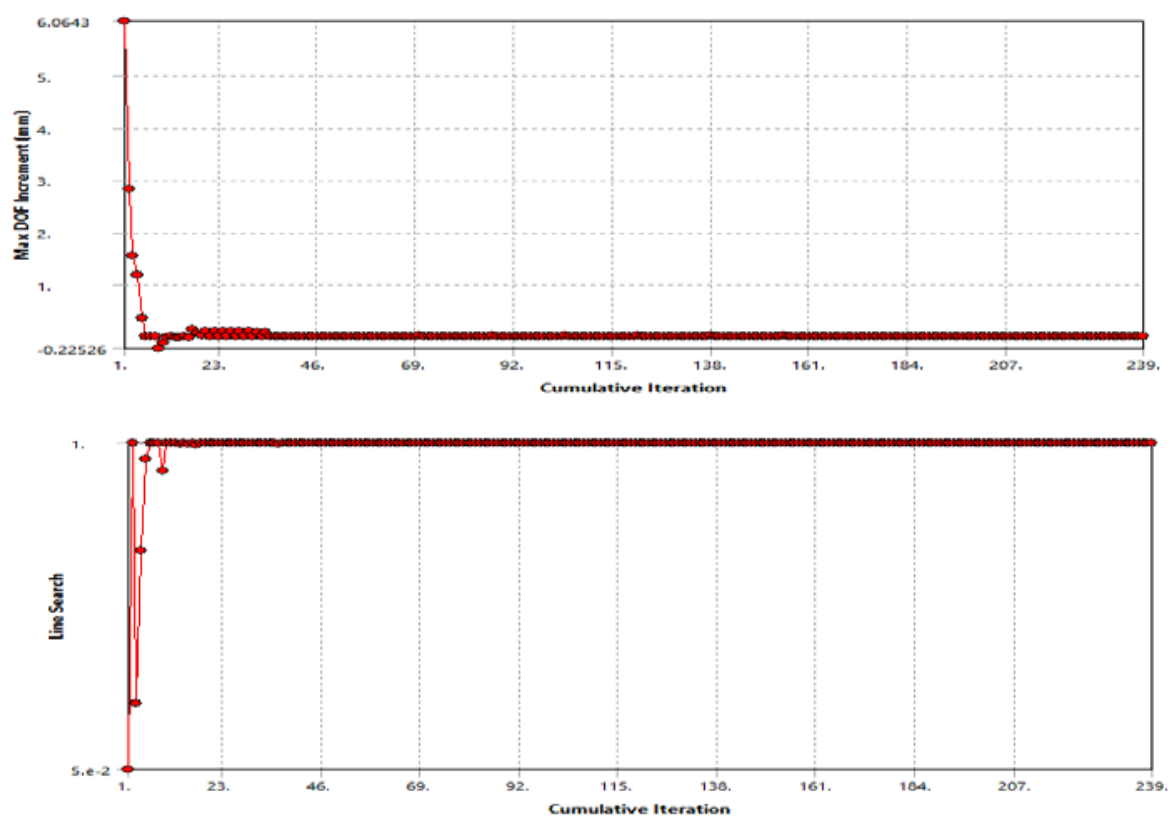

Figure 38: Max DOF Increment (upper) and Line search (lower) against cumulative iterations for line fit P3G connection

From Figures 39 through 50, the green plot shows the maximum value of the parameter and the red plot shows the minimum value of the parameter at various load steps. The stress values at the start and the end position of the bending load are the same owing to the symmetrical location in the connection. The variation of maximum von Mises stress in the hub and the shaft, maximum contact stress, sliding distance, and torsional shear stress in shaft throughout the load steps are shown respectively from Figures 39 through 43 for P3G connection. It can be observed that there is a major change in magnitude during the first two load steps due to an increase in load from zero to full torsional load at the end of first load step and application of bending load at the end of the second load cycle. After that, the values are almost consistent. 


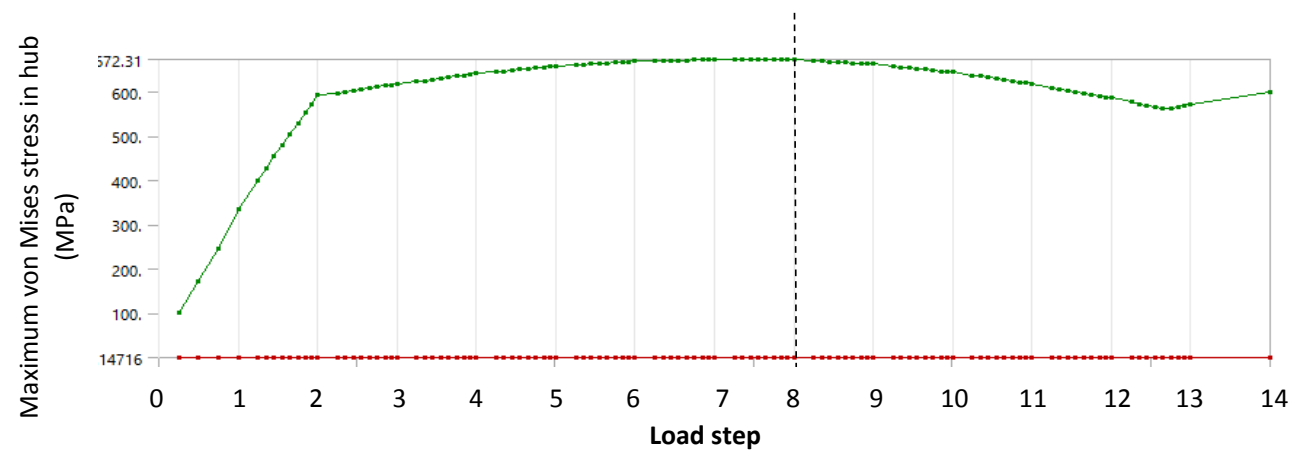

Figure 39: Variation of maximum von Mises stress on the P3G hub

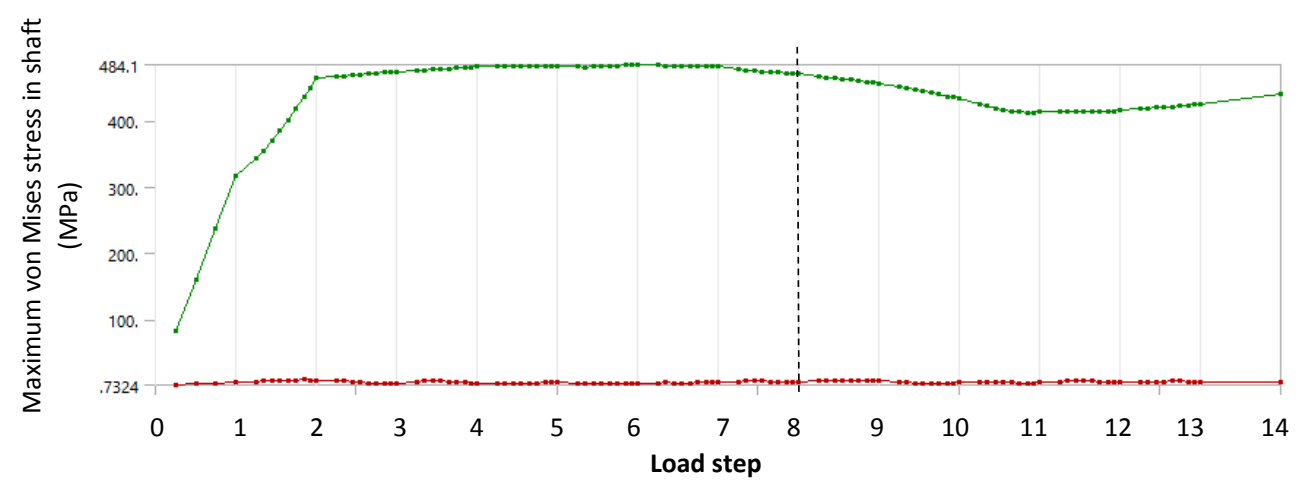

Figure 40: Variation of maximum von Mises stress on the P3G shaft

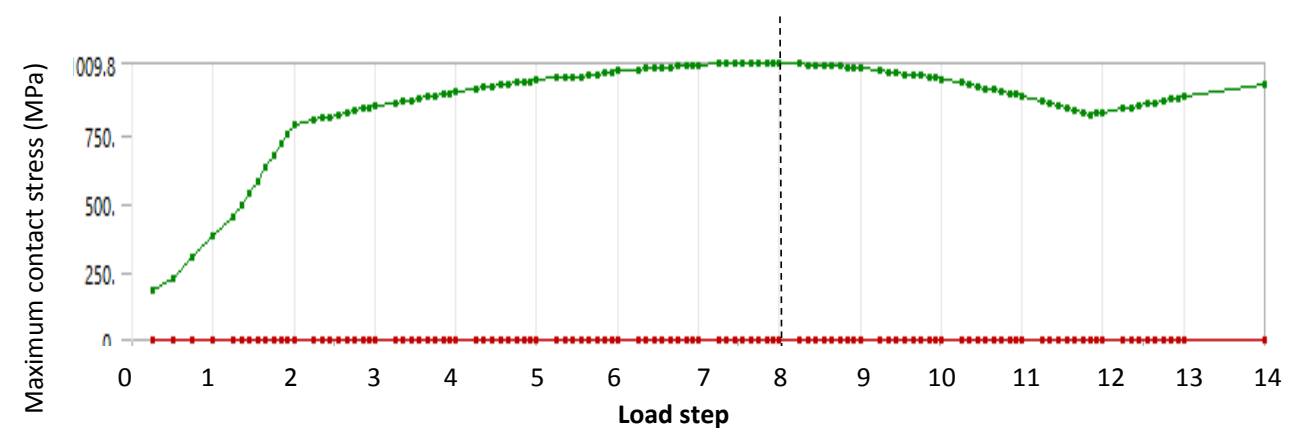

sure 41: Variation of maximum contact stress in P3G shaft hub connection

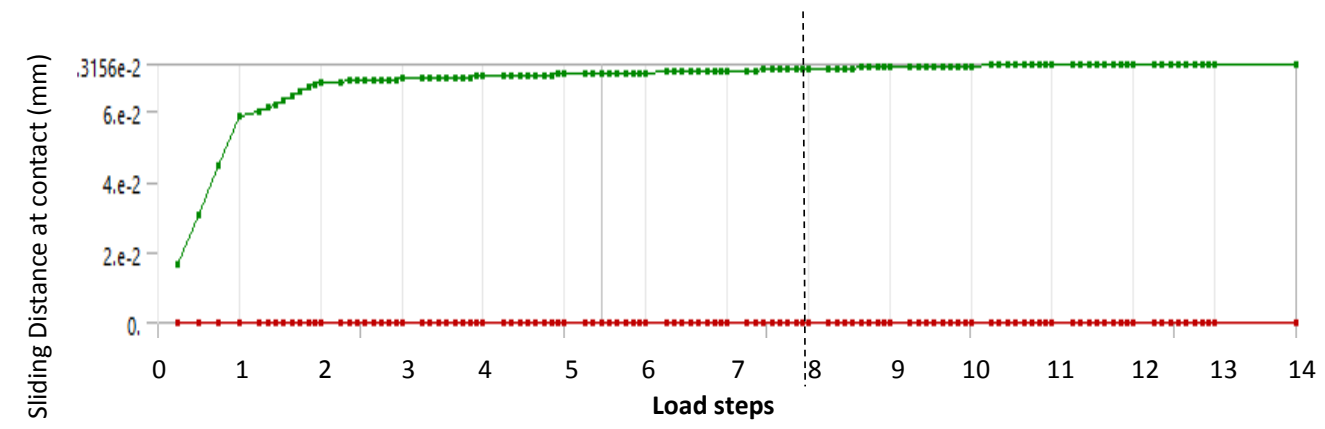

Figure 42: Variation of sliding distance in P3G shaft hub contact region 


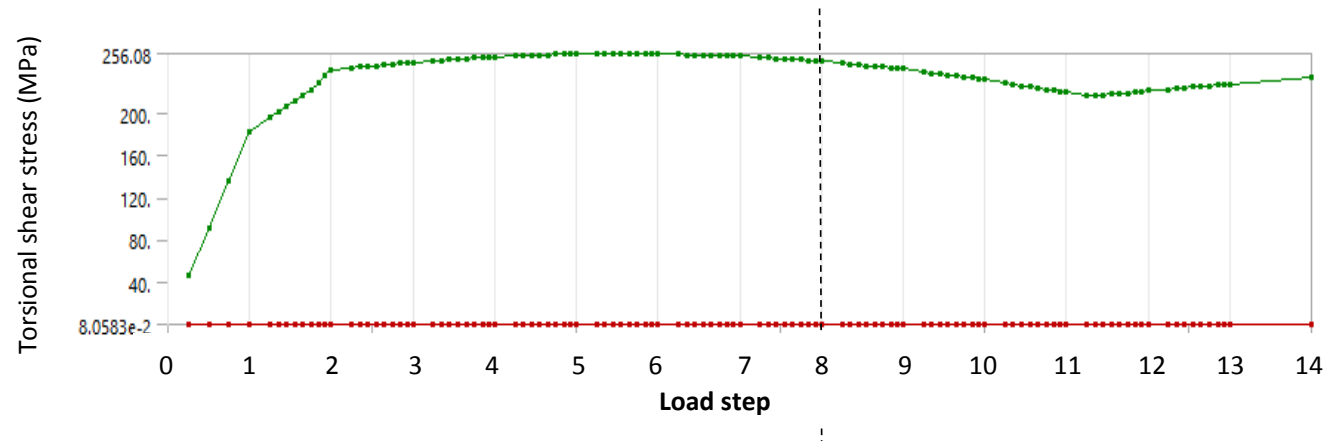

Figure 43: Variation in torsional shear stress of P3G shaft

The middle dotted black line is the line of symmetry for P3G profile i.e. the apex of the profile. From Figure 39, it can be observed that the maximum von Mises stress is greatest near the apex of the profile. The von Mises stress is lower at the first and the last position that correspond to the flat middle portion of the profile. For the shaft in Figure 40, the von Mises stress is found to be the highest just before the apex in steps 5 and 6 , because they have the radial force corresponding to the position where the contact between the shaft and the hub occurred. Due to the expansion of the hub as a result of the contact stress, the load steps 11 and 12 act in the position of expansion and has the lowest von Mises and contact stress as seen from Figure 41. This shows that contact stress is the major contributor of von Mises stress. The sliding distance is initially increased due to an increase in load and became consistent after load step 2, due to sticking as a result of friction as seen from Figure 42. The maximum torsional shear stress on the shaft follows the same trend as the von Mises stress of the shaft as expansion of the hub caused lesser shear stress to develop in load steps 11 and 12 as seen from Figure 43.

Based on the value of the maximum von Mises stress in connection, the critical load step has been taken for load analysis and is listed in Table 10. 


\subsection{2) Transient Loading for P4C connection}

The load step for P4C polygonal profile in clearance fit is shown in the upper plot of Figure 44, where the first load step is with a ramped pure torsional load of $80 \mathrm{Nm}$ (shown by the blue line), which is then kept constant for rest of the load step. From the second load step, a constant magnitude bending load is applied, varying in position along the circumference of the hub. The load position is radially varied at $10^{\circ}$ and covers the full $90^{\circ}$ range for $\mathrm{P} 4 \mathrm{C}$ profile with a total of 11 load steps. The convergence can be seen in the lower plot of Figure 44. The line search and maximum DOF increment can be seen in Figure 45 and it can be observed that the maximum DOF increment is smaller than the maximum DOF increment in the P3G transition fit connection and this solution could have been obtained without the line search scaling as well.
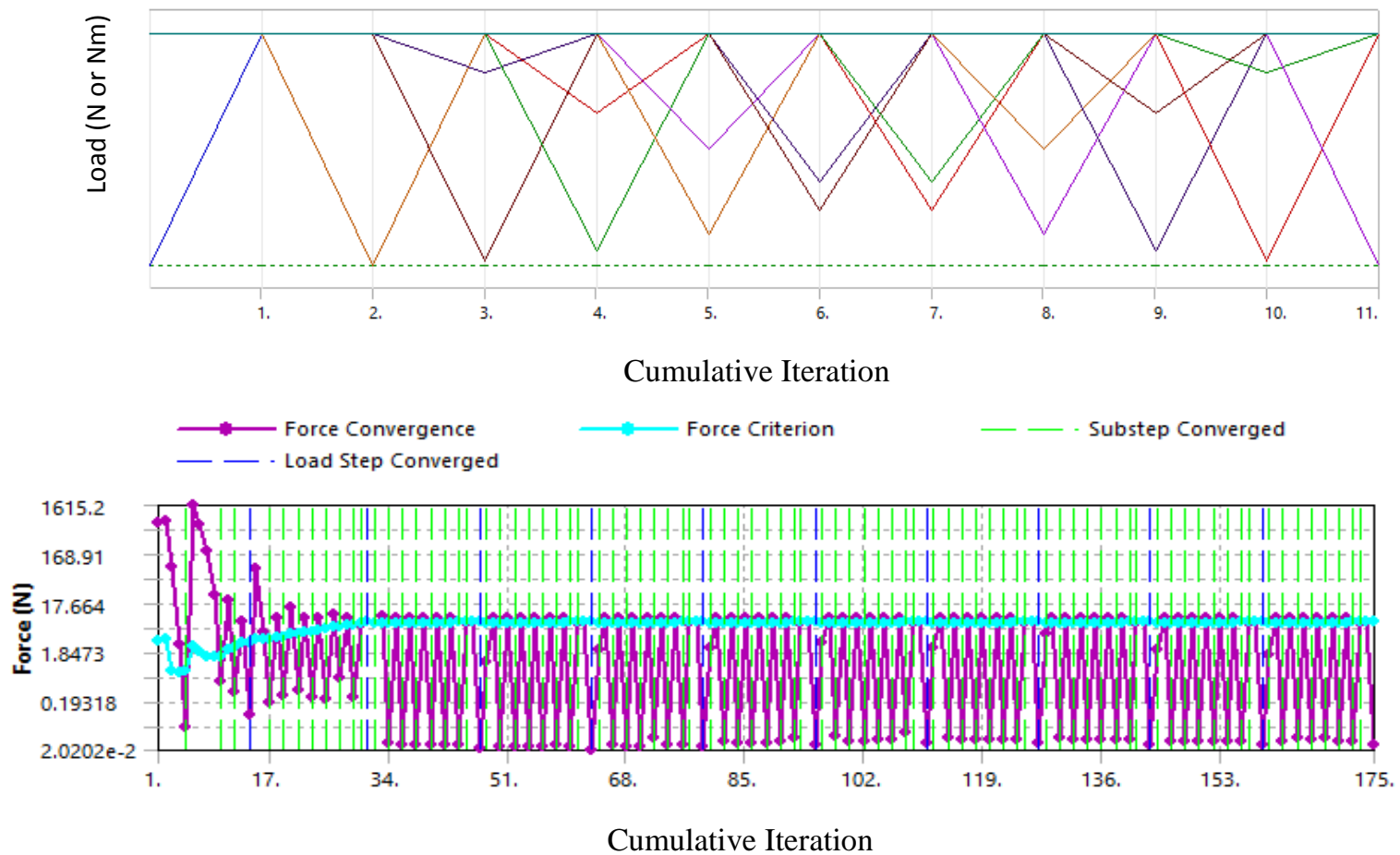

Figure 44: Load step (upper) and convergence (lower) for clearance fit P4C connection 

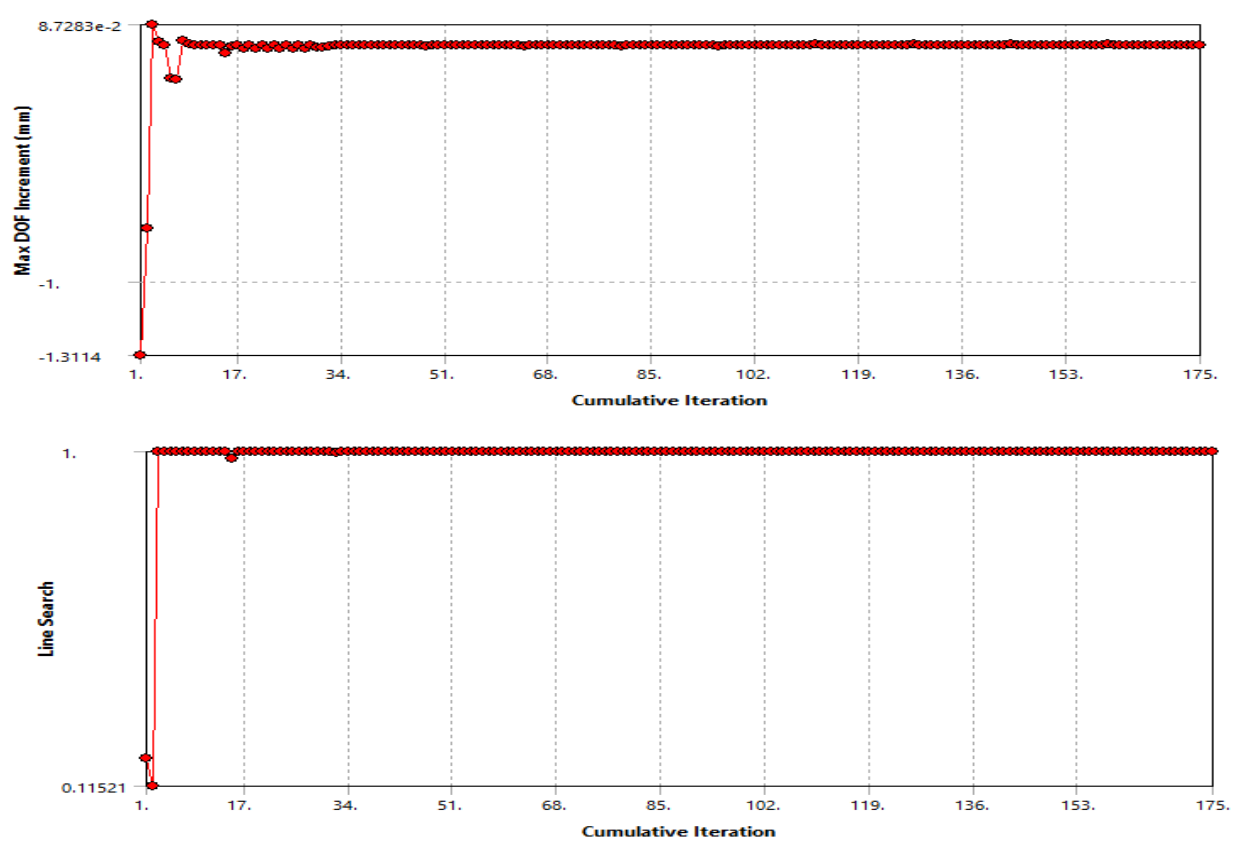

Figure 45: Max DOF Increment (upper) and Line search (lower) against cumulative iterations for clearance fit P4C connection

The variation between maximum von Mises stresses in hub and shaft, maximum contact stress, sliding distance, torsional shear stress in shaft are shown for P4C connection from Figures 46 to 50 respectively. The two middle dotted black lines in these plots show the load step corresponding to $30^{\circ}$ and $60^{\circ}$, which correspond to the position of change in the continuous profile curve.

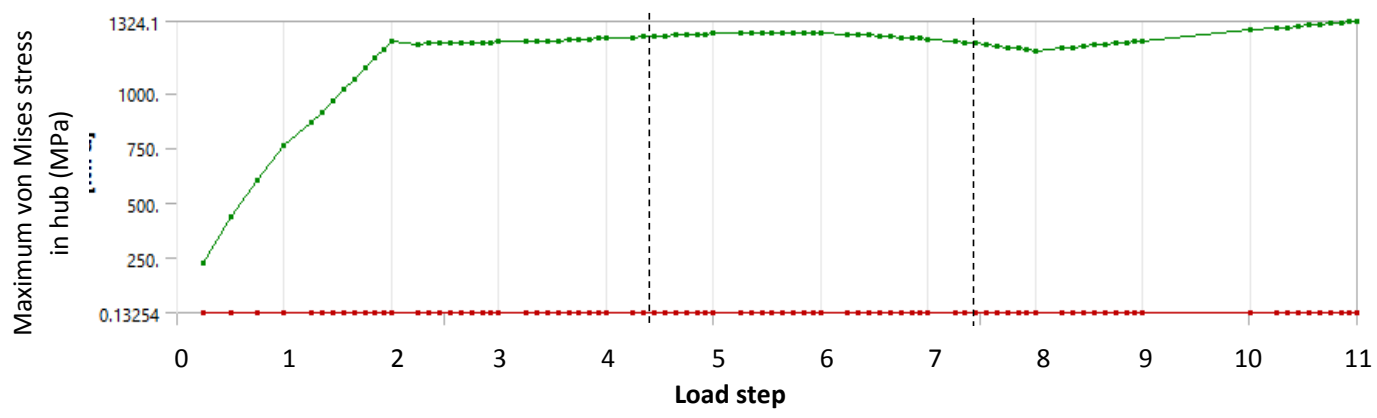

Figure 46: Variation of maximum von Mises stress on the P4C hub 


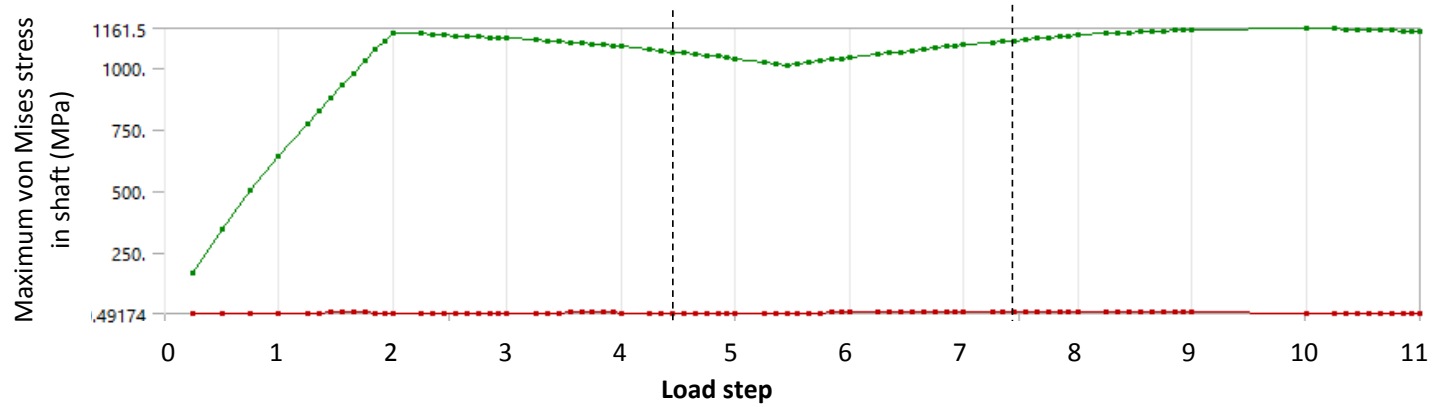

Figure 47: Variation of maximum von Mises stress on the P4C shaft

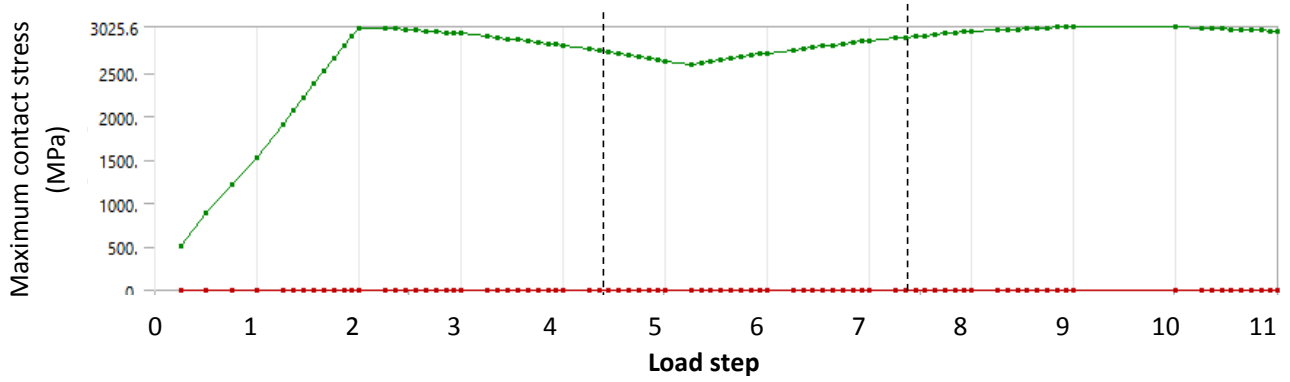

Figure 48: Variation of maximum contact stress in P4C shaft hub connection

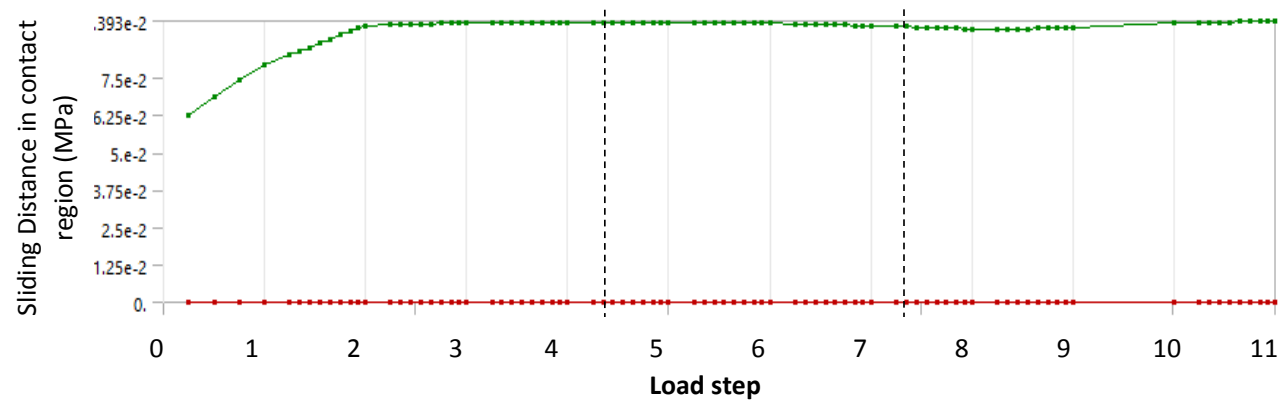

Figure 49: Variation of sliding distance in P4C shaft hub contact region

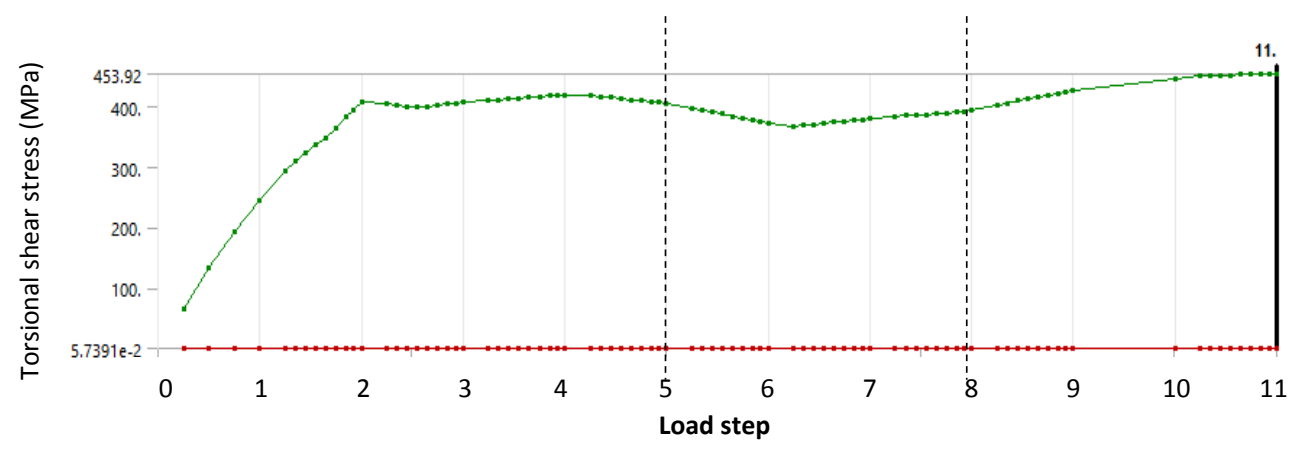

Figure 50: Variation in torsional shear stress in P4C shaft 
From Figure 46, it can be observed that the hub has almost consistent maximum von Mises stress distribution, except for load step 8. Load step 8 corresponds to the contact region of the shaft and hub. The reason for lower stress is the nearby concentrated contact in clearance fit causing the expansion of hub in that position. Figures 47,48 , and 50 , show the trend of variation of maximum von Mises stress, contact stress, and torsional shear stress to be similar. The region of minimum stress is the one where the bending load is on the circular portion of the curve. The bending load acts as a pivot to expand the hub near the contact and the maximum stress is lower in that load step. The second and the last load step are symmetrical positions, which correspond to the maximum stress and have been used in Table 10 for the final analysis. The sliding distance is consistent after the second load step when the ramping of second load step ends as seen in Figure 49.

\subsection{3) The Effect of Non linearity}

The contact between two bodies is a nonlinear phenomenon. When load is applied to two bodies in contact or near contact, the contact status between constituent bodies changes. If the contact status is initially closed, i.e. touching each other, the load will cause the contact area to either increase or decrease. If the contact between two bodies is initially open, i.e. there is a gap between the two bodies as in a clearance fit, the contact will be closed as a result of loading. The relation between the force and the displacement is not proportional for these contact problems since deformation changes with load and the solution is nonlinear. For example, in the case of clearance fit the contact area will increase from zero to the final equilibrium area. A nonlinear increase in the stress may occur depending upon the steepness of the load ramping as the force and contact area increase at their own rate. For the current polygonal connections, referring to Figures 46 and 47, it can be observed that the von Mises stress increases during the first load 
cycle when the torsional stress rises from 0 to $80 \mathrm{Nm}$. Due to the faster rise in force magnitude than rise in contact area, the increase is steady and approximately linear. Also, the maximum stress in the second load step after the bending force is ramped up, increases with the load in a similar steady way. From Figure 51, it can be observed that the contact status is initially sliding (value 2) for the torsional load. This means that the tangential force is higher than the frictional force allowing sliding of the bodies. As the bending load is applied, the normal contact pressure rises, which increases the frictional resistance. As a result, sticking occurs in some portion of the connection as shown in the upper plot of Figure 51. It is worth noting that the sliding contact status in the first load step is due to the ramping in load. If the moment is kept constant after the first ramping, the contact status changes to sticking as the body will be in equilibrium.

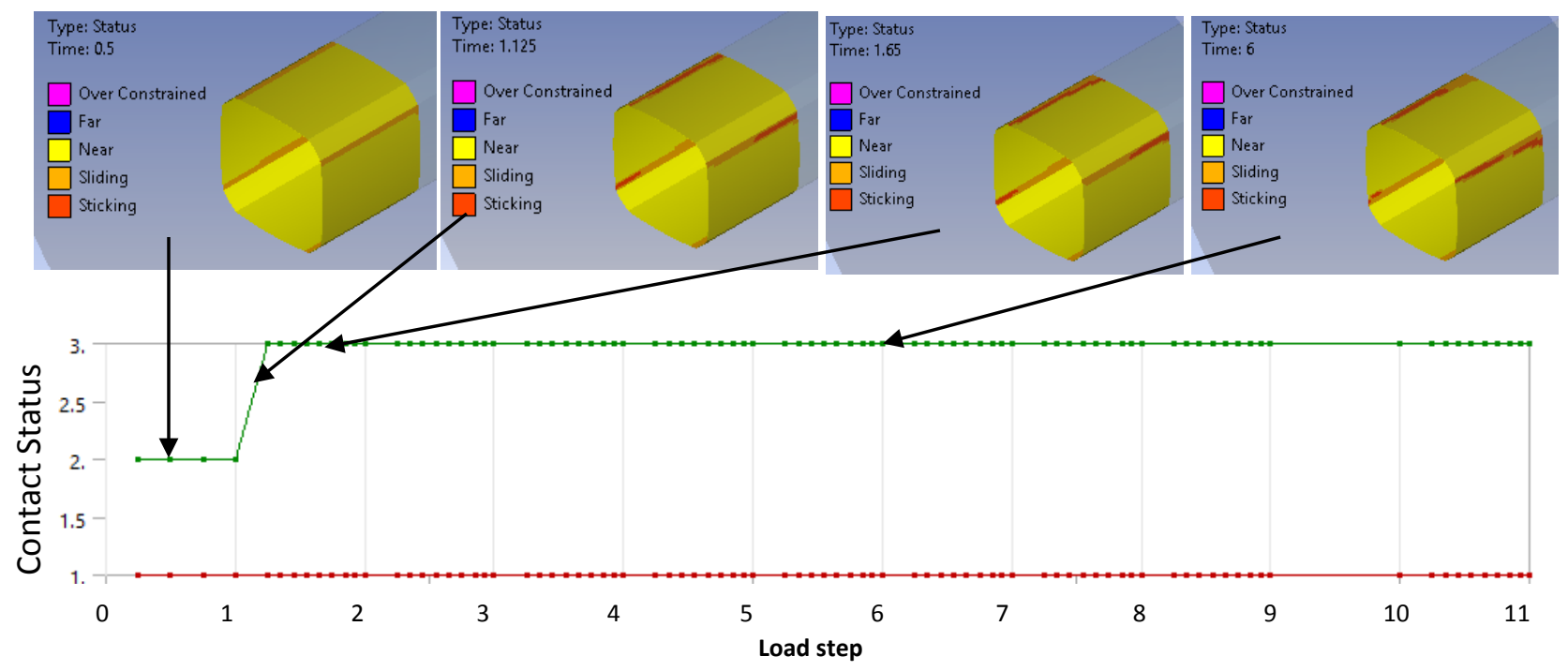

Figure 51: Plot of contact status for P4C clearance fit connection

Similar analysis applies to all other fits of P3G and P4C shaft hub assembly and the results of all the transition loading is shown in Table 10. The position leading to the maximum stress is the critical position in terms of strength and the position varies by fit and profile. The position of maximum stress is shown in Table 10. Using the results from Table 10, finite element analyses 
of low stress torsional bending load were performed without performing the full transient solution to save computational time.

Table 10: Position corresponding to maximum stress

\begin{tabular}{|r|r|r|}
\hline Profile & Fit & Position from Figures 14 and 15 \\
\hline P3G & Clearance & $5\left(30^{\circ}\right.$ from and above apex $)$ \\
\hline P3G & Transition & 8 (apex $)$ \\
\hline P3G & Interference & $9\left(10^{\circ}\right.$ from and below apex $)$ \\
\hline P4C & Clearance & 2 or 11 (vertical or horizontal $)$ \\
\hline P4C & Transition & 2 or 11 (vertical or horizontal $)$ \\
\hline P4C & Interference & $8\left(30^{\circ}\right.$ from and above apex $)$ \\
\hline
\end{tabular}


4.3) Loading capacity under torsional and torsional bending load

The result of torsional bending and pure torsional loading on three and four lobe polygonal shaft and hub connections for each fit has been explained under this section. The result has been presented for three loading conditions, viz. torsional load of $80 \mathrm{Nm}$, torsional bending load case 1 (torsion of $8 \mathrm{Nm}$ and bending load of $410 \mathrm{~N}$ applied at the hub) and torsional bending load case 2 (torsion of $80 \mathrm{Nm}$ and bending load of $4600 \mathrm{~N}$ applied at the hub).

The DIN standard provides the geometry and loading strength for P3G and P4C profiles under torsional load. In the limiting cases, when the length of the shaft is small, the effects of bending load can be neglected and the system can be analyzed using the pure torsion calculation. The drive shaft can be an application of pure torsional loading. The earlier studies of Czyzewski and Odman (1988), Huang et al. (2010) and Lü and Liu (2011) was limited to in-plane strain analysis of torsional load only. The schematic of torsional loading applied can be seen from Figure 52 and the result of the loading is presented in Table D1 for P3G and Table D2 for P4C connection of Appendix D.

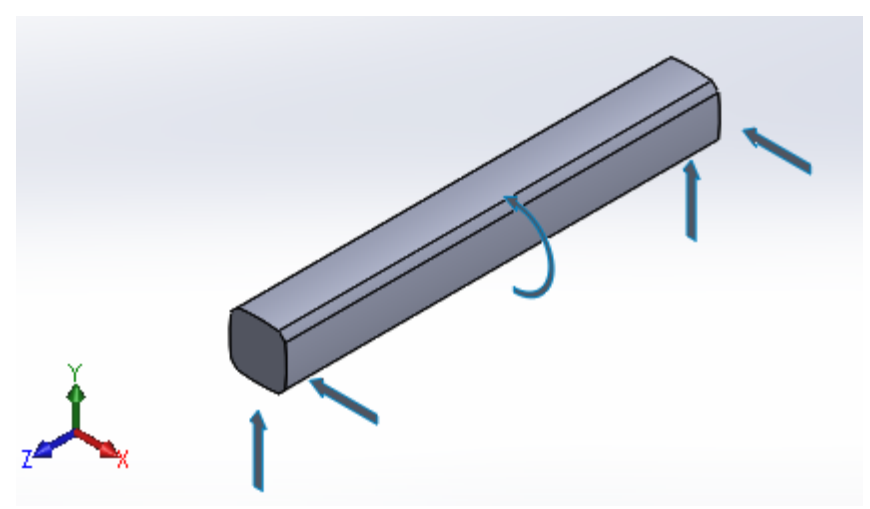

Figure 52: Loading for pure torsional load of $80 \mathrm{Nm}$ on P4C shaft (hub not shown)

A shaft is mostly subjected to a torsional bending load and mere comparison from the torsional strength is inadequate since bending load is also significant, especially for longer shafts. The current industrial application of polygonal shafts is limited to heavy torque 
transmission in a press fit for P3G profiles and low torque sliding fit applications for P4C profiles. In the current work, a comparison has been made between the loading capacities in various fits for a constant stock size. The FEA result of torsional bending load for case 1 and 2 for various fit and profiles are presented in Table D3 through Table D12 under Appendix D. The schematic of the loading is shown in Figure 53.

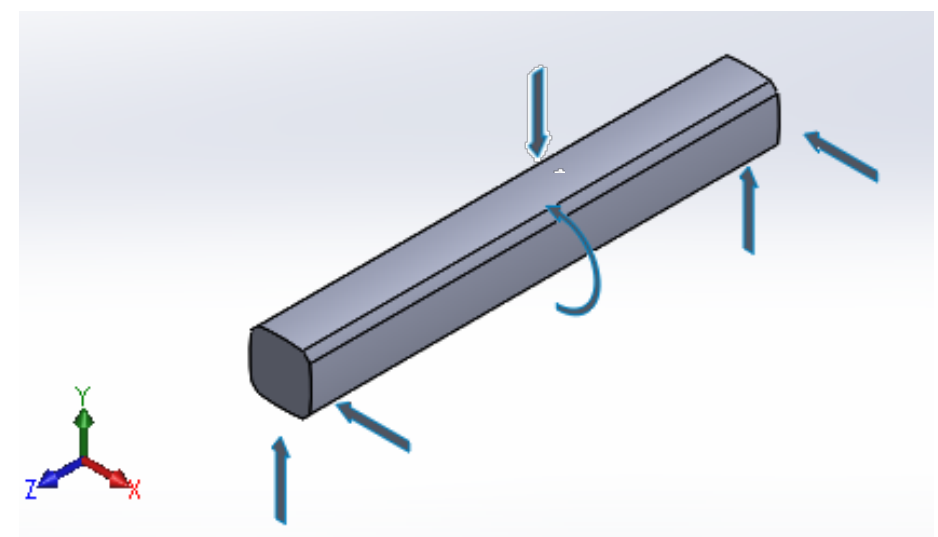

Figure 53: Loading for torsional bending load on P4C shaft (hub not shown)

The result of the loading on various stresses are shown graphically from Figure D1 to D32 in Appendix D and are explained as follows.

\subsection{1) Effect of loading on Torsional Shear stress}

The maximum torsional shear stress developed in a P3G and P4C polygonal shaft away from the contact point for various fits have been presented for three loading conditions, i.e. pure torsional load and the two torsional bending load.

i) Pure torsional load

The variation of the torsional shear stress for the three fit of P3G and P4C shafts are shown in Figure 54 and it can be seen that the stress developed in P4C shafts is more than the P3G shaft. It is worth noting that the magnitude of the stress is not significant different with the 
percentage difference between the highest stress (P4C clearance fit) to the lowest stress (P3G interference fit) being $8 \%$.

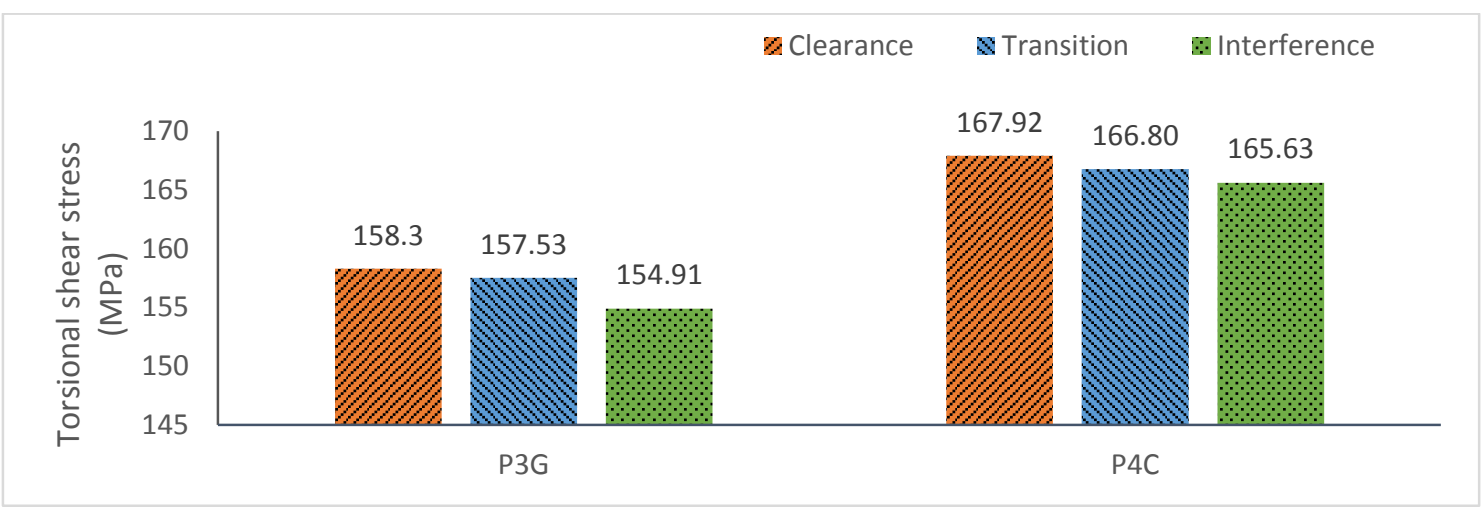

Figure 54: Torsional shear stress for torsional load of $80 \mathrm{Nm}$

ii) Torsional Bending Load

The torsional shear stress developed in the torsional bending load case 1 is shown in Figure 55 and torsional bending load case 2 is shown in Figure 56. The result from the torsional bending follows the same trend from torsional load, i.e. P4C has an average torsional shear stress higher than the P3G. The difference in the magnitude between the highest stress (P4C clearance fit) to the lowest stress (P3G interference fit) is $8.89 \%$ for loading case 1 and $14.83 \%$ for loading case 2. The transition fit has the lowest stress for both profiles for loading case 1 , but the difference is below $1 \mathrm{MPa}$ to infer any conclusion.

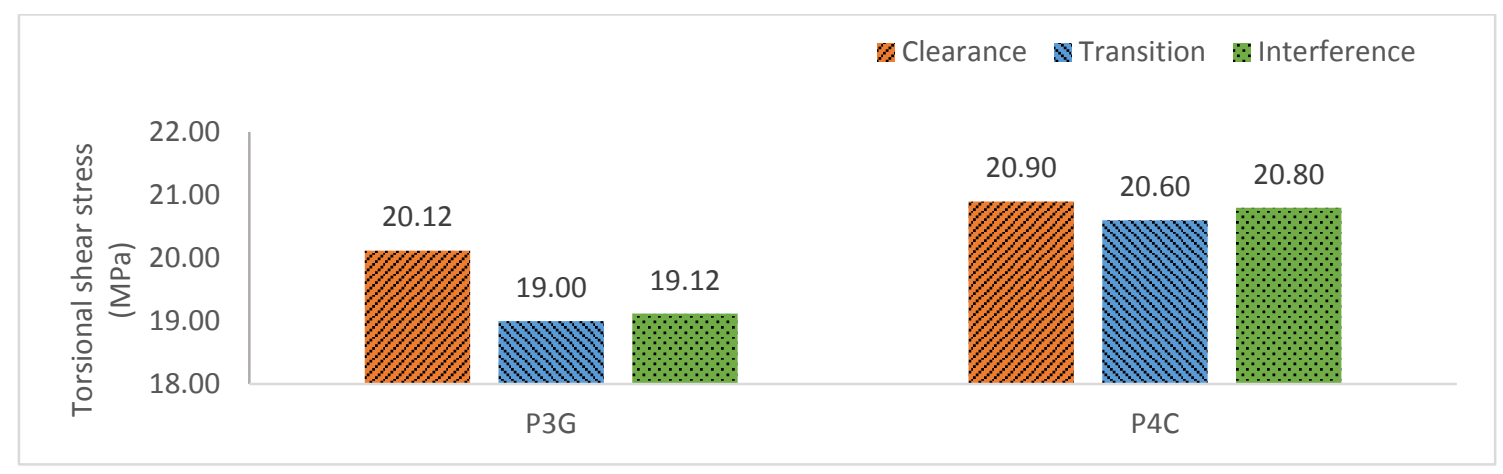

Figure 55: Torsional shear stress for torsional bending load case 1 


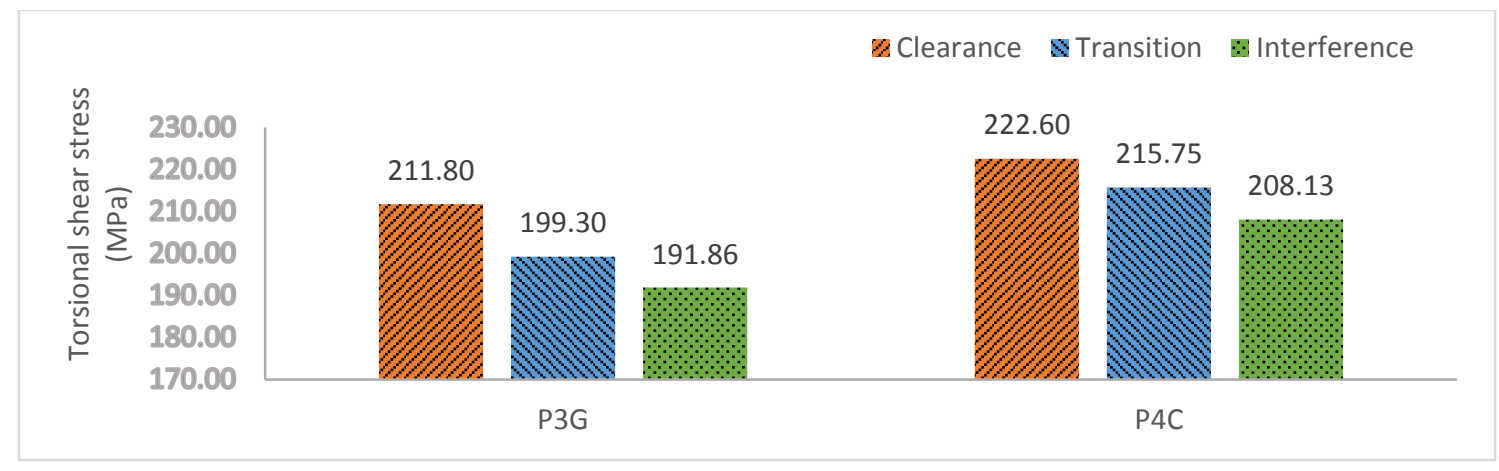

Figure 56: Torsional shear stress for torsional bending load case 2

From the result of torsional shear stress, the difference between the maximum stress and minimum stress developed is found to be less than $15 \%$ in all loading cases and the difference between the values for the same fit is below $9 \%$ to make any decision on the suitability of a particular profile or fit.

\subsection{2) Effect of loading on Normal stress along axial direction}

When torsional bending load is applied to a polygonal shaft, the normal stress arises along the axis due to the bending load on the shaft and warpage due to torsion on a noncircular section. The direction of application of torsion causes the hub to move in a certain direction, which is restricted by the friction and the interference from the material interface, leading to a complex three dimensional state of stress, even in pure torsional load. If the middle of the shaft if considered i.e. on the neutral axis, the effect of the bending load is nullified and the normal stress is only due to warping. The variation on the normal stress along the axis for the torsional load is shown in Figure 57. As evident from the figure, the interference fit has the highest stress as it opposes any relative movement of shaft and hub, while the clearance and the transition fit have lower stresses. The figure also shows the lowest normal stress in clearance fit for both profiles. Among the clearance fit, the stress is lowest in P4C connection. 


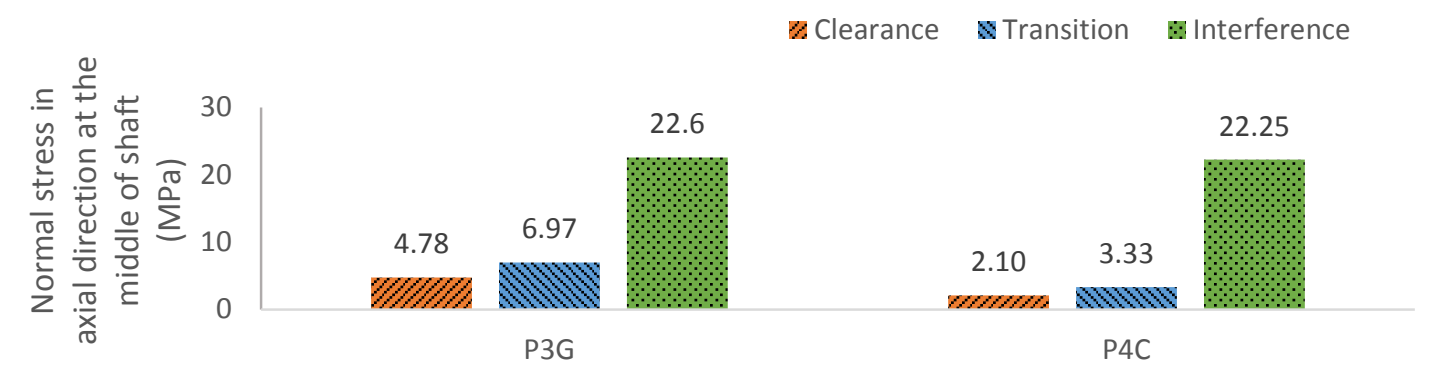

Figure 57: Normal stress in axial direction at the middle of the shaft subjected to torsional load

Results of the torsional bending loading case 1 and 2, shown in Figure 58 and 59

respectively, show that the normal stress is highest in the interference fit as in the pure torsional load since the body resist any warping. The lowest stress is found in transition fit for loading case 2. The stress is found to be lower in P4C connection than the P3G connection as in the pure torsional load due to smaller tangential force resulting in lower axial component after warping.

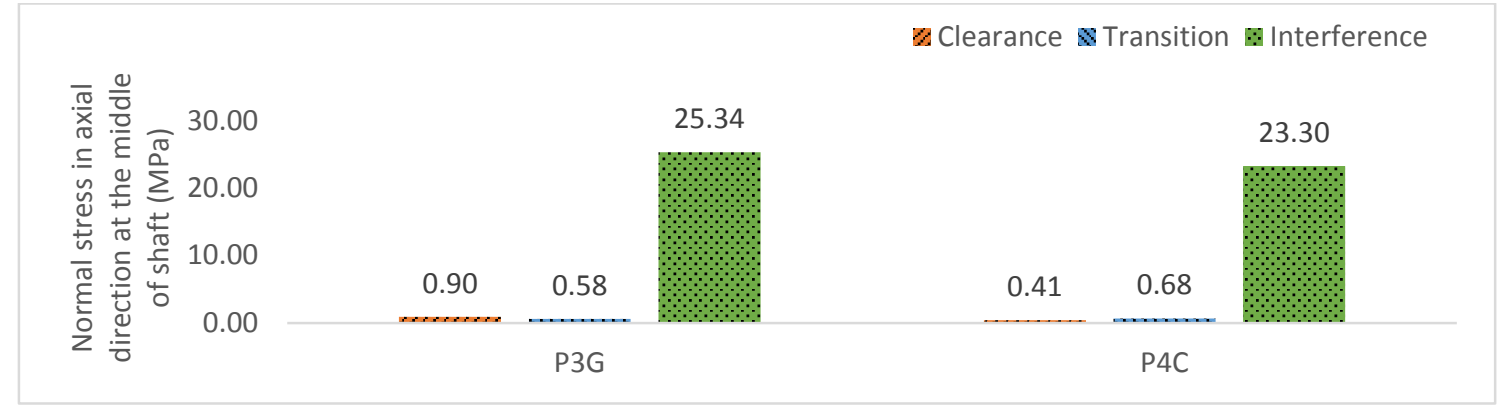

Figure 58: Normal stress in axial direction at the middle of the shaft subjected to torsional bending load case 1

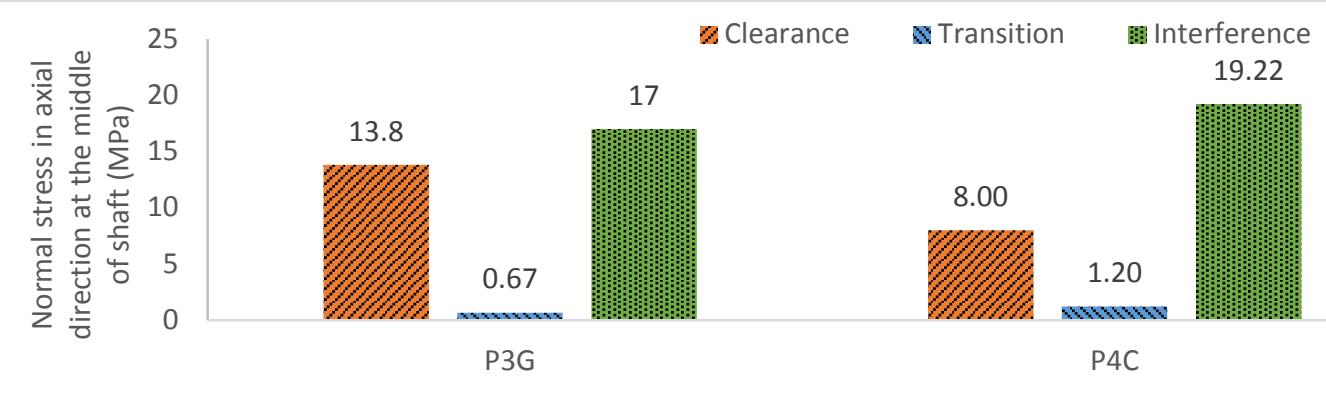

Figure 59: Normal stress in axial direction at the middle of the shaft subjected to torsional bending load case 2 
The maximum axial displacement of the hub for the torsional bending load are shown in Figure 60 and 61 for loading case 1 and 2 respectively.

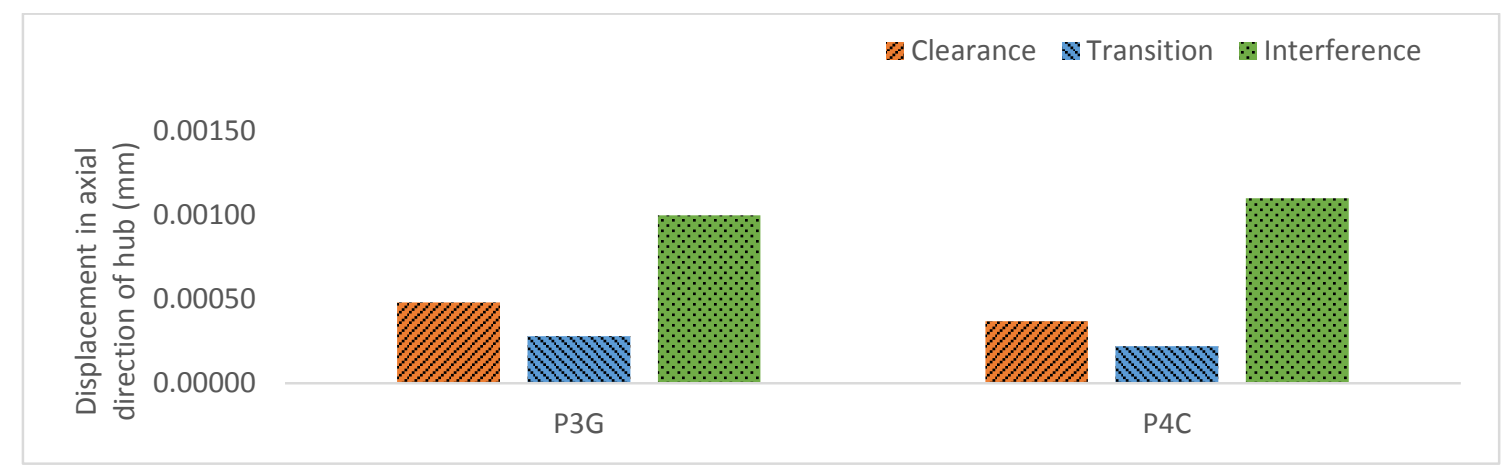

Figure 60: Maximum Axial displacement of hub (mm) for loading case 1

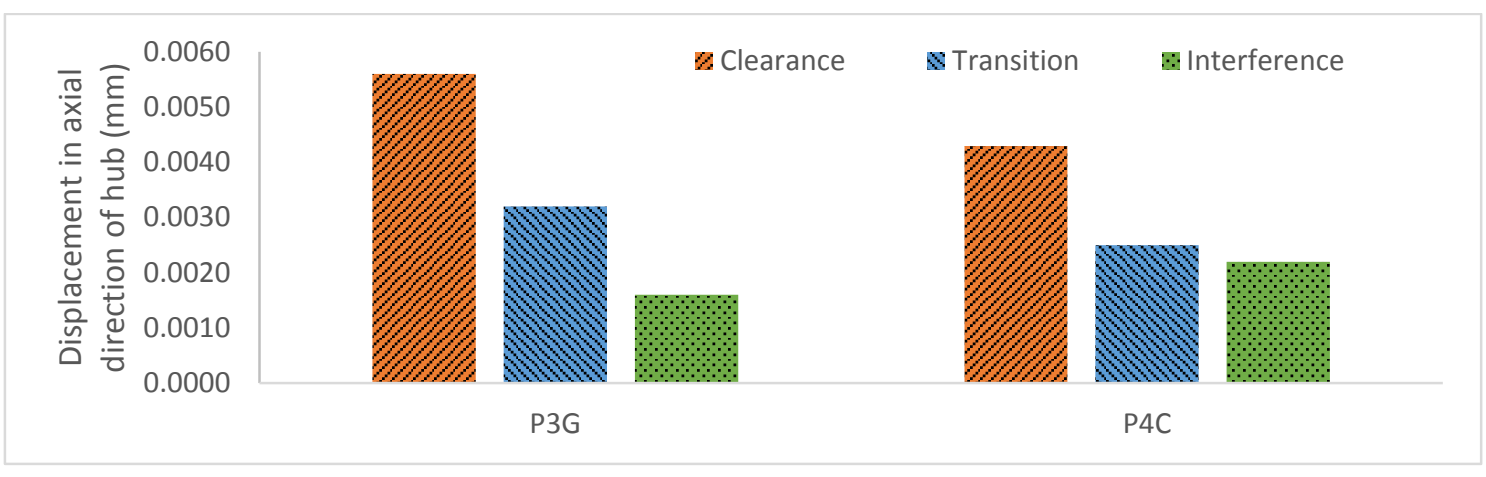

Figure 61: Maximum Axial displacement of hub (mm) for loading case 2

Loading case 1 shows the least hub displacement in transition fit followed by clearance fit. The loading case 2 shows the least hub displacement in clearance fit followed by transition fit. Among the P3G and P4C clearance fit connections, the P4C shaft has lower normal stress and lower hub displacement in all torsional bending cases. This is explained due to the higher pressure angle in the P4C profile. A higher pressure angle gives lower tangential force and when the shaft warps, the axial force is smaller. This is the advantage of the $\mathrm{P} 4 \mathrm{C}$ clearance fit, which can be used for sliding fit connection as it has the least normal stress and hub displacement, although the von Mises stress is larger. This result supports the finding of Winterfeld (2001), who mentioned suitability of P4C connections for sliding fit. 


\subsection{3) Effect of loading on Contact Stress}

The major contributor to the maximum von Mises stress in a polygonal shaft is the contact stress in terms of magnitude and distribution (verified in transient loading section). The variation in the maximum contact stress in the P3G and P4C profiles are shown in Figure 62 for pure torsional load. The P4C shaft is found to have greater contact stress than the P3G shaft. The reason for greater contact stress in the P4C shaft is smaller contact area due to a sharper edge at the discontinuity of the epitrochoidal curve and the circle, which digs into the hub leading to much higher stress than the harmonious P3G profile.

The magnitude of the contact stress is very high compared to the normal and torsional shear stress (more than 10 times torsional shear stress in P4C clearance fit) as seen from Tables D1 to D12 in Appendix D.

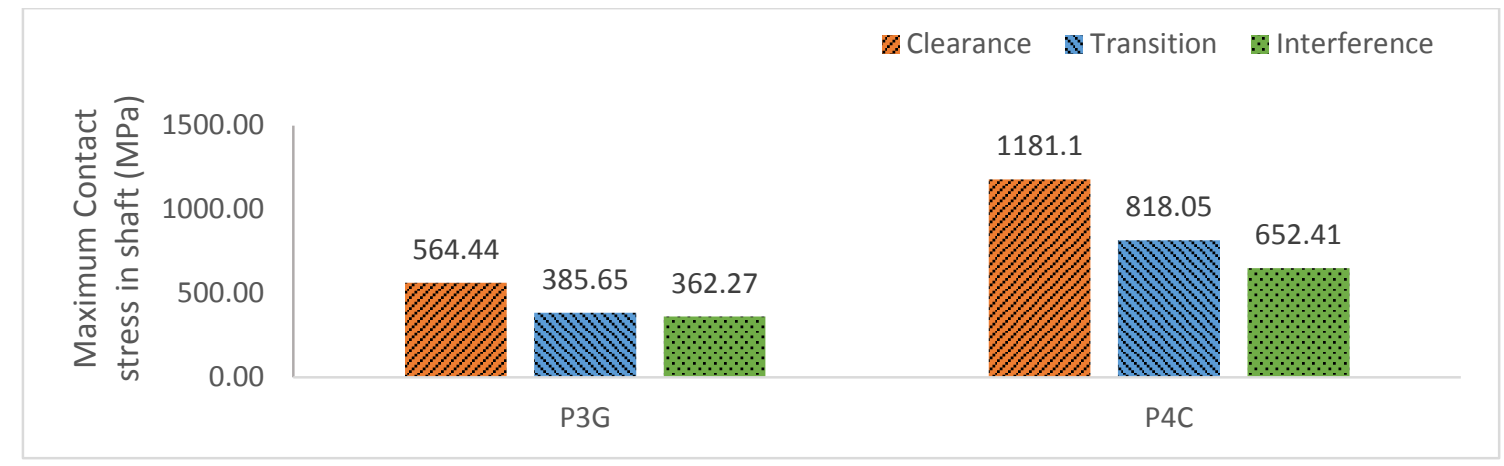

Figure 62: Maximum contact stress for P3G and P4C shafts subjected to pure torsional load

Figure 63 shows the highest contact stress in the clearance fit, followed by transition fit. The reason for greater stress in clearance fit is the contact area available to transmit the force. The clearance fit has the smallest contact area, leading to higher contact stress. Although, the transition fit should have the whole connected area as contact area, the application of the torsion causes local expansion of the hub leading to smaller contact area in three regions in P3G profile and four regions in $\mathrm{P} 4 \mathrm{C}$ profile. 
The variation of the maximum contact stress for torsional bending load case 1 is shown in Figure 63. The loading case 1 has highest stress in the interference fit. This is due to the interference pressure itself as the contact pressure is lower. The transition fit has the lowest stress since it has no interference pressure and has higher contact area than the clearance fit. It is worth noting that the difference in contact stress is only $6.12 \%$ between the transition fit of P3G and P4C profile for loading case 1 . This infers that the stress developed are similar for smaller loads.

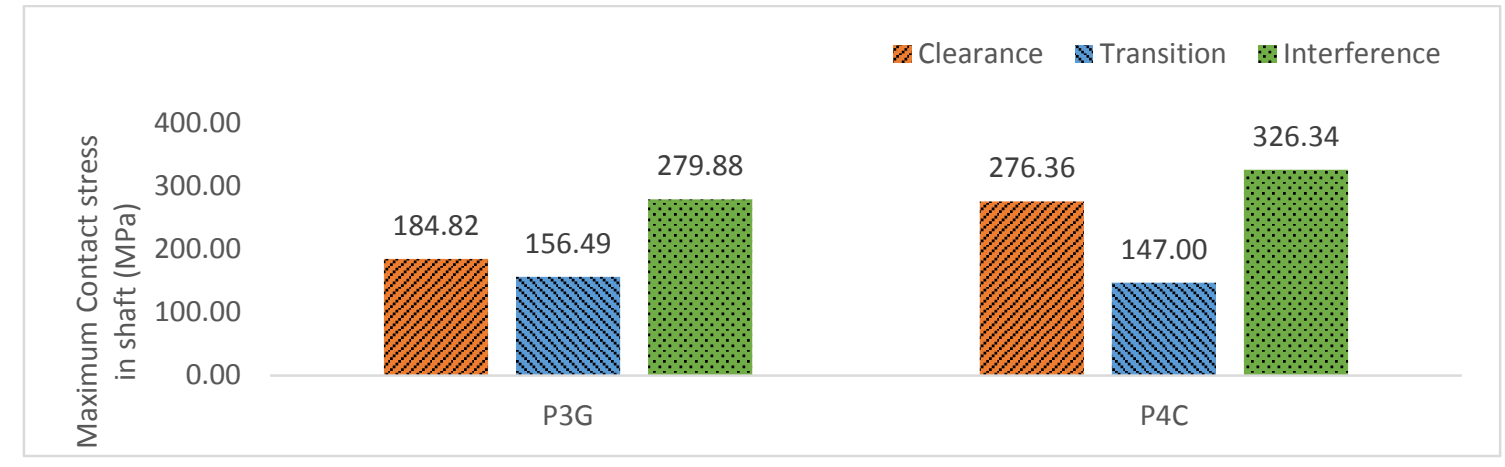

Figure 63: Maximum contact stress for P3G and P4C shafts subjected to torsional bending load case 1

The variation of maximum contact stress for torsional bending load case 2 is shown in Figure 64. The variation in the contact stress is similar to the pure torsional loading that shows higher stress in clearance fit and P4C shafts and lowest stress in interference fit and P3G shafts. This shows that torsional load is the major contributor in the design of polygonal shafts and supports the analyses of previous writers, who used pure torsional load for analysis. The difference between the contact stress of the clearance and the interference fit is $46 \%$ in P3G profile and $80.6 \%$ in $\mathrm{P} 4 \mathrm{C}$ profile. This is a very steep rise in contact stress in P4C profile as the gap is increased. Even within the clearance fit, the percentage difference is $67 \%$ between the P3G and P4C connection. 

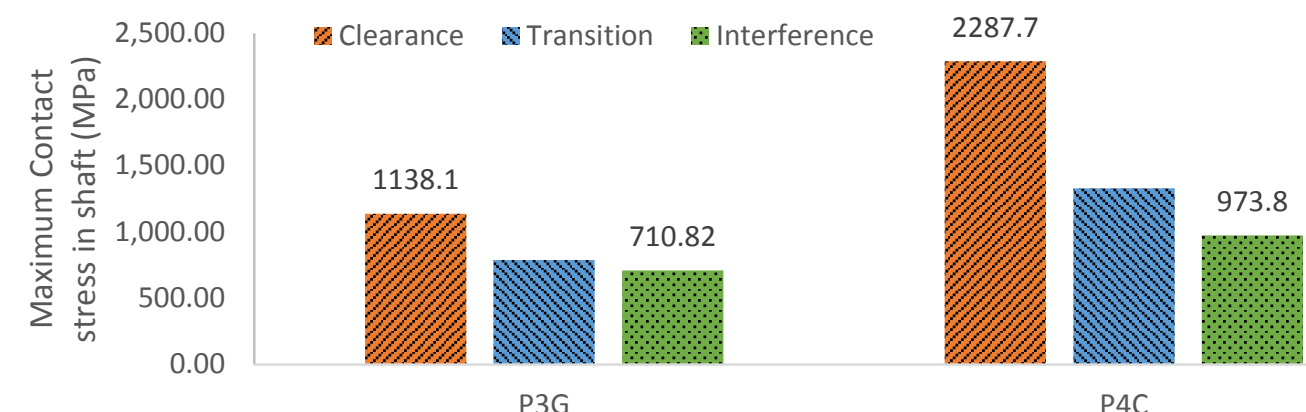

P4C

Figure 64: Maximum contact stress for P3G and P4C shafts subjected to torsional bending load case 2

Before making a decision based on the maximum contact stress, the average contact stress also needs to be analyzed. Figure 65 shows the average contact stress for torsional bending loading case 2.

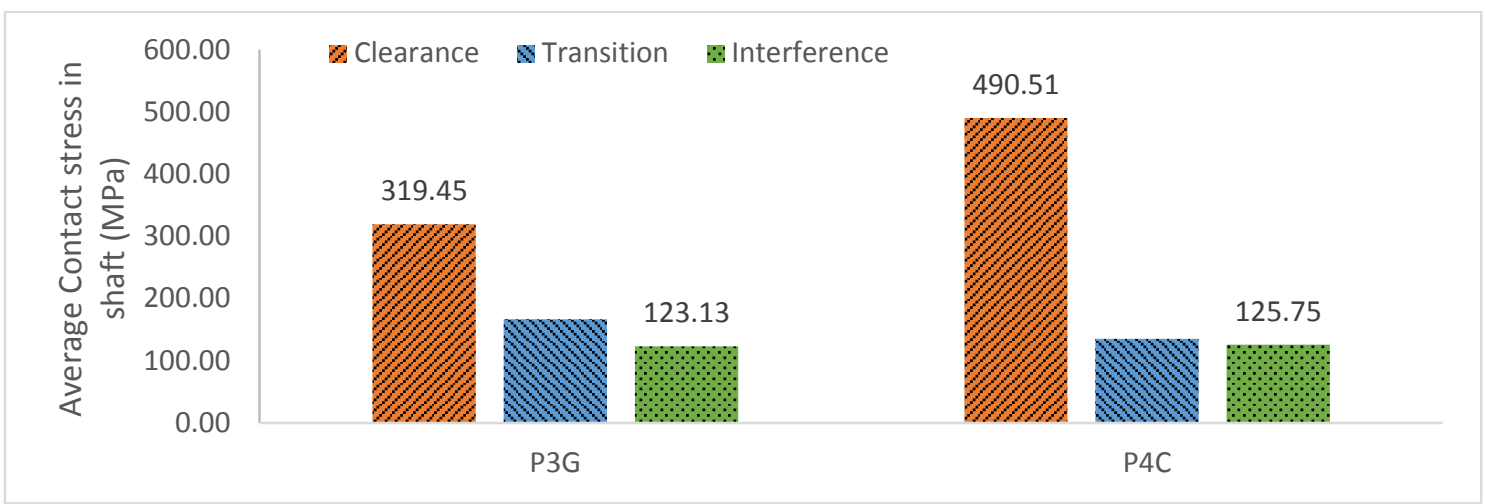

Figure 65: Average contact stress for P3G and P4C shafts subjected to torsional bending load case 2

Figure 65 shows the same trend as maximum contact stress, i.e. maximum contact stress in P4C and clearance fit and lower in P3G and interference fit. This shows unsuitability of the clearance fit P4C profiles for higher stress applications as the values are higher than the yield stress of common shaft materials as steel and will cause local plastic deformation, which will lead to premature failure. The interference fit has the lowest stress and is the recommended fit 
for high torsional bending load. The reason for the lower stress in interference fit is the expansion of the hub as a result of the stress, which causes the contact pressure to decrease.

Figures 66 and 67 show the contact status of the P3G and P4C shaft and hub connection under torsional bending load case 2 .

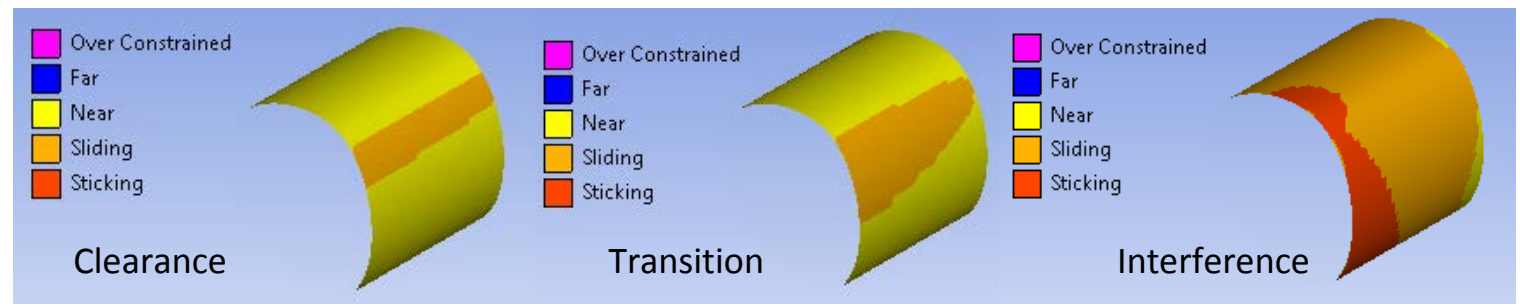

Figure 66: Contact status in P3G profile for loading case 2

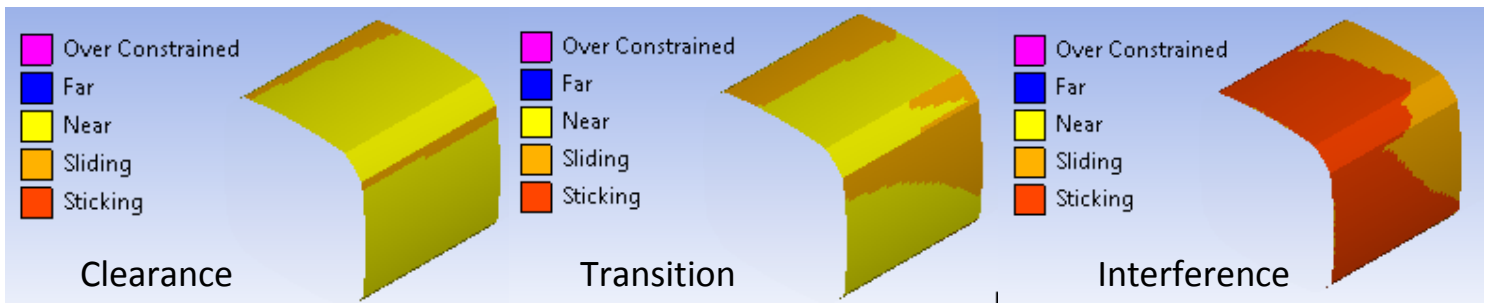

Figure 67: Contact status in P4C profile for loading case 2

From Figure 66, it can be observed that the contact area is small in clearance fit and looks like a rectangular strip. Due to the local deformation by the applied stress, the contact region in transition fit is also localized, although it is larger than clearance fit. Similar results can be seen from the contact status for P4C polygonal shafts from Figure 67 for clearance and transition fit. The contact status for clearance fit is similar to the results found by Czyzewski and Odman (1988) for pure torsional in-plane stress condition. For the interference fit, most of the contact status is sticking in P4C and sliding in P3G connection. This is due to larger contact pressure in P4C shaft leading to larger frictional force. 


\subsection{4) Effect of loading on Contact pressure Distribution}

As determined from the earlier sections, the major stress for a polygonal shaft and hub connection is the contact pressure. The conformal contact between the shaft and the hub exclude the use of Hertzian contact pressure theory. Studies by Czyzewski and Odman (1988) verified that the contact distribution does not follow the Hertzian contact principle except in low torque load and larger clearance. This section explores the contact stress distribution for a torsional load and a torsional bending load for various fits conditions. Earlier studies were conducted in pure torsional load, using a plane strain condition for clearance fit and did not analyze the 3D loading condition. The pure torsional load will have three identical contact zones for P3G and four identical contact zones for P4C and any one of them can be used for analysis. For the torsional bending load, the contact pressure is due to the bending load and the torsional load. This loading will have one maximum contact stress region and other lower contact stress regions.

i. Contact Pressure Distribution for Pure Torsional Load

For a torsional load of $80 \mathrm{Nm}$, the distribution of the contact stresses for clearance, transition and interference fit for P3G shaft are shown in Figure 68. The contact stress distribution has been plotted against the angle from the contact origin and end position and corresponding part in profile is shown below it. It can be inferred that the total angle of contact is smaller for a clearance fit and larger for an interference fit. The contact areas in a clearance fit do not touch each other before loading and contact in a small region after loading, unlike the interference fit where the contact is initially in all the common regions. Figure 68 shows that all the curves are skewed and the distribution is not symmetrical. The distribution istriangular for clearance fit, similar to the results of Czyzewski and Odman (1988). A small crest and trough are found alongside a big peak for interference fit unlike the clearance fit, which has only one peak. 
The transition fit has a stress distribution between the two other extreme fits, with a larger contact region than clearance fit and smaller than interference fit. This shows that the contact pressure variation cannot be approximated with a Hertzian cylindrical contact. The smaller crest in the interference zone is due to interference itself and the trough in the middle due to the deformation of the area near the contact point caused by expansion of the hub and less stress. The deformation of the hub causing decrease in contact stress in the middle is similar to the result of Czyzewski and Odman (1988).
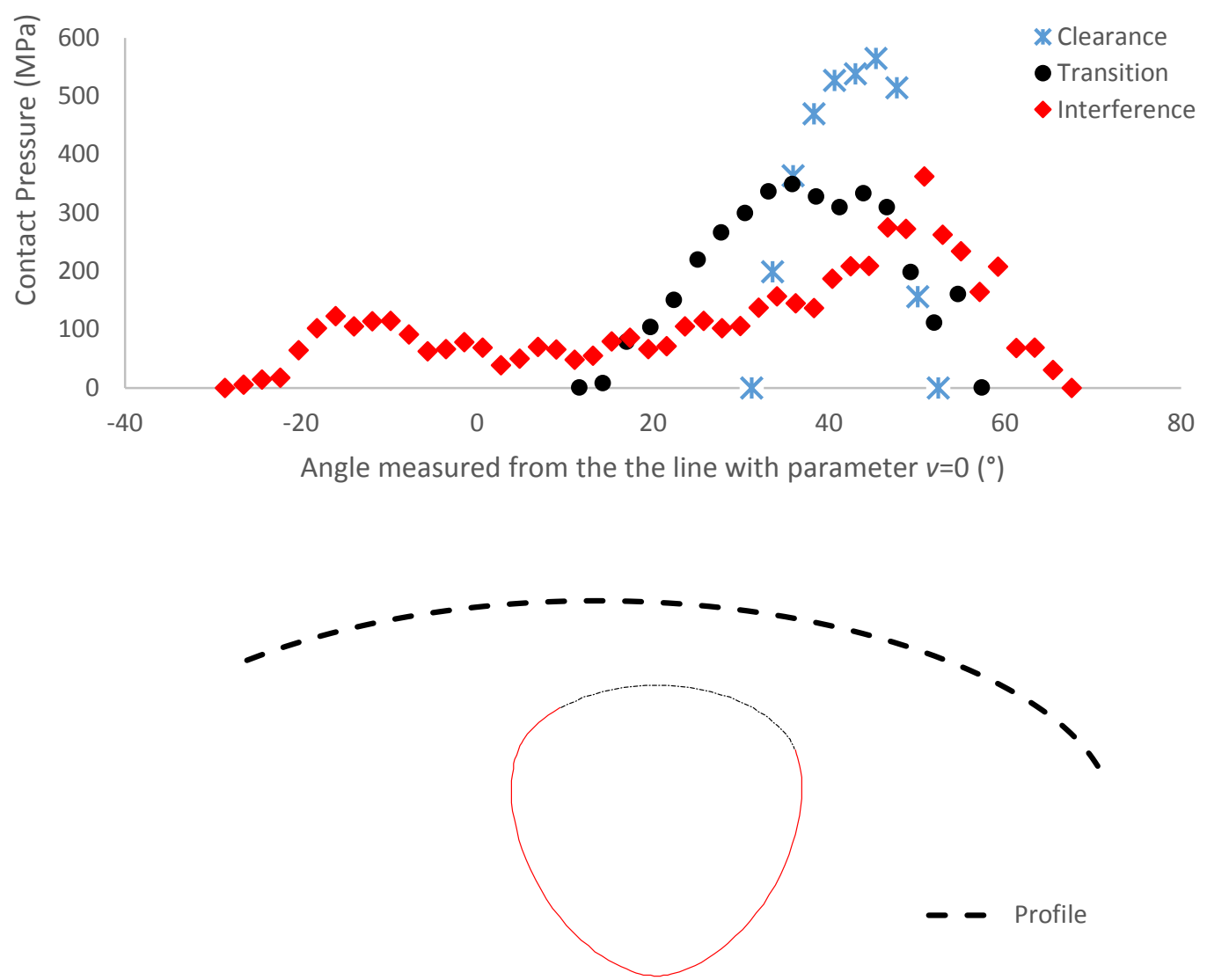

Figure 68: Contact Stress distribution for P3G profile for torsional load of $80 \mathrm{Nm}$

The contact stress distribution for the P4C shaft is shown in Figure 69 for pure torsional loading. The curves are skewed as in the P3G connection, depending upon the direction of the torque. The clearance fit has the smallest contact region, smaller than the P3G clearance fit as 
well. The contact distribution for transition fit has a large crest around $35^{\circ}$ and trough in the middle and has a small crest at around $22^{\circ}$. The reason for this is expansion of the hub near the peak area causing the dip in pressure nearby. Also, the lack of close nodes near the contact area causes the sudden rise in pressure. The contact pressure distribution in interference fit of P4C connection is similar to the P3G interference fit. The contact region in the P3G interference fit is larger than the P4C interference fit. This is due to higher contact angle developed in the P4C region that separates the interference zone. The maximum stress occurs around same angle for all fits in P3G and P4C shaft. The slight difference is due to direction of torque and corresponding slip.

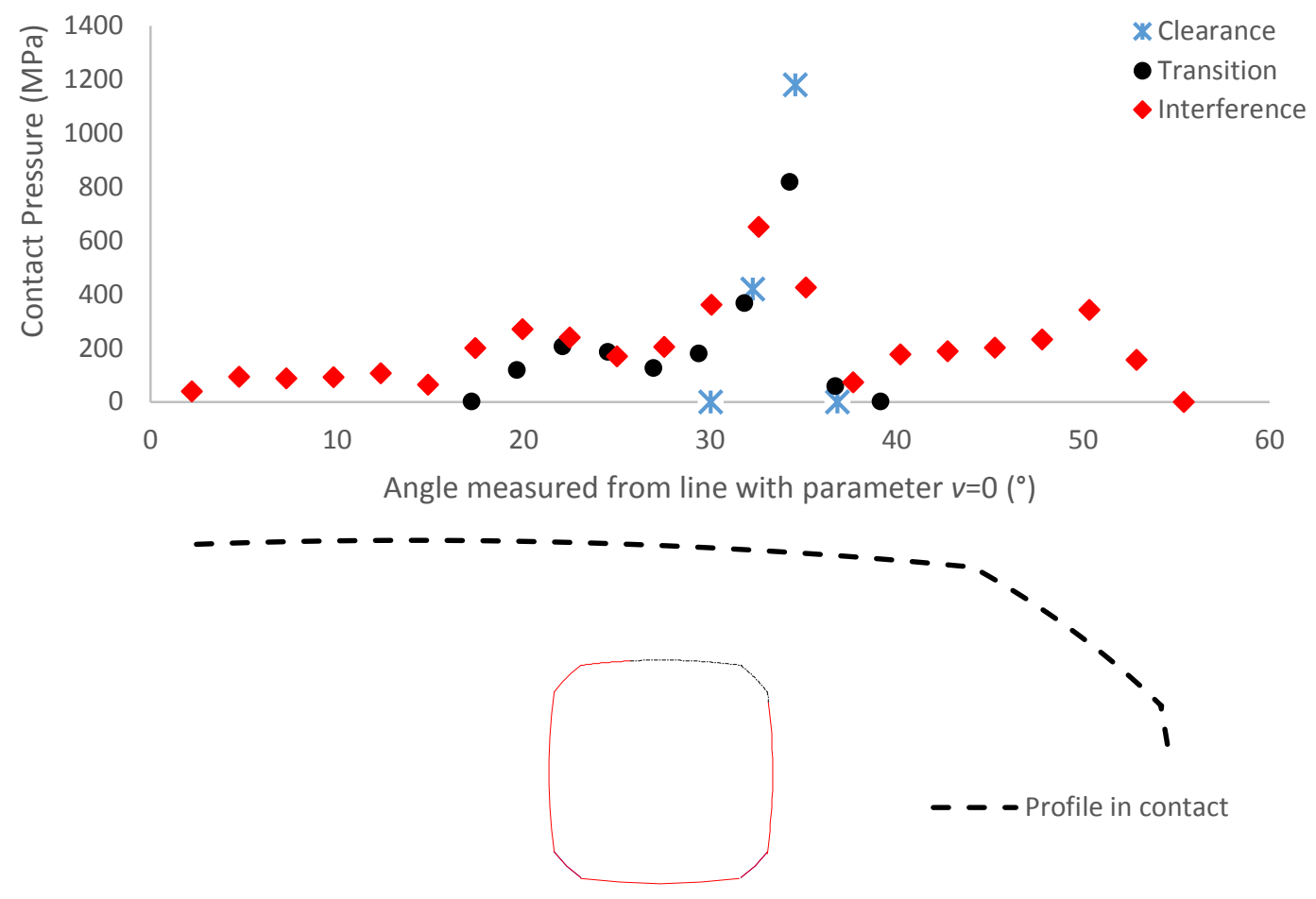

Figure 69: Contact Stress distribution for P4C profile for torsional load of $80 \mathrm{Nm}$

ii. Contact Pressure for Torsional Bending Load

For a torsional load of $80 \mathrm{Nm}$ and bending load of $4600 \mathrm{~N}$, the distribution of the contact stress for line to clearance, transition, and interference fit for P3G shaft are shown in Figures 70, 
71 and 72 respectively. The contact stress distribution has been chosen for the region with maximum contact stress and the values are much lower in other contact regions, unlike equal values in pure torsional load.

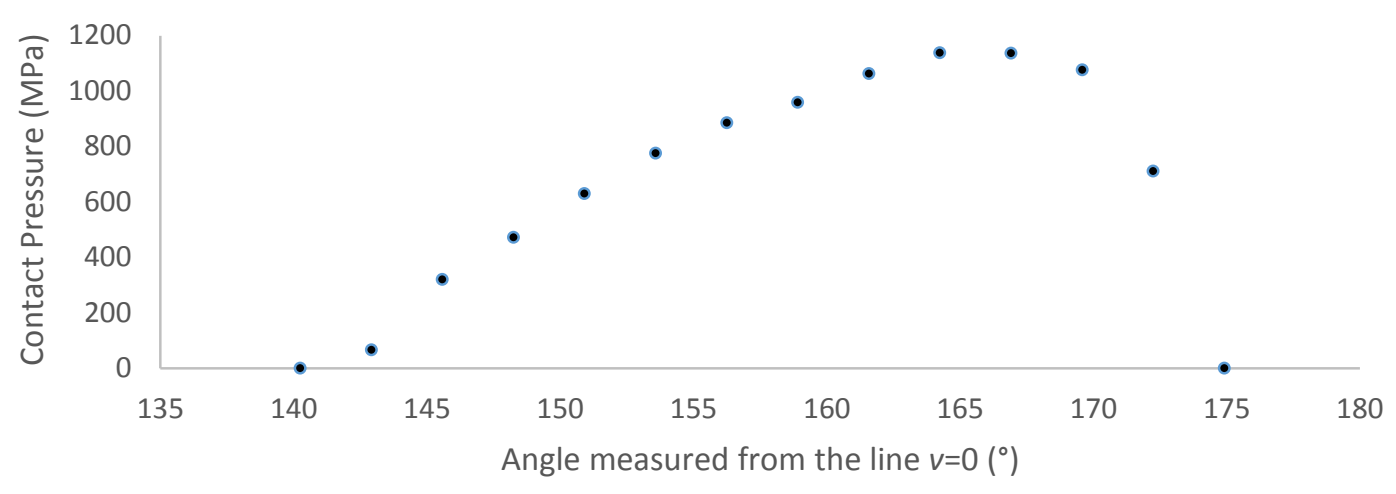

Figure 70: Contact Stress distribution for P3G clearance fit for torsional load of $80 \mathrm{Nm}$ and bending load of $4600 \mathrm{~N}$

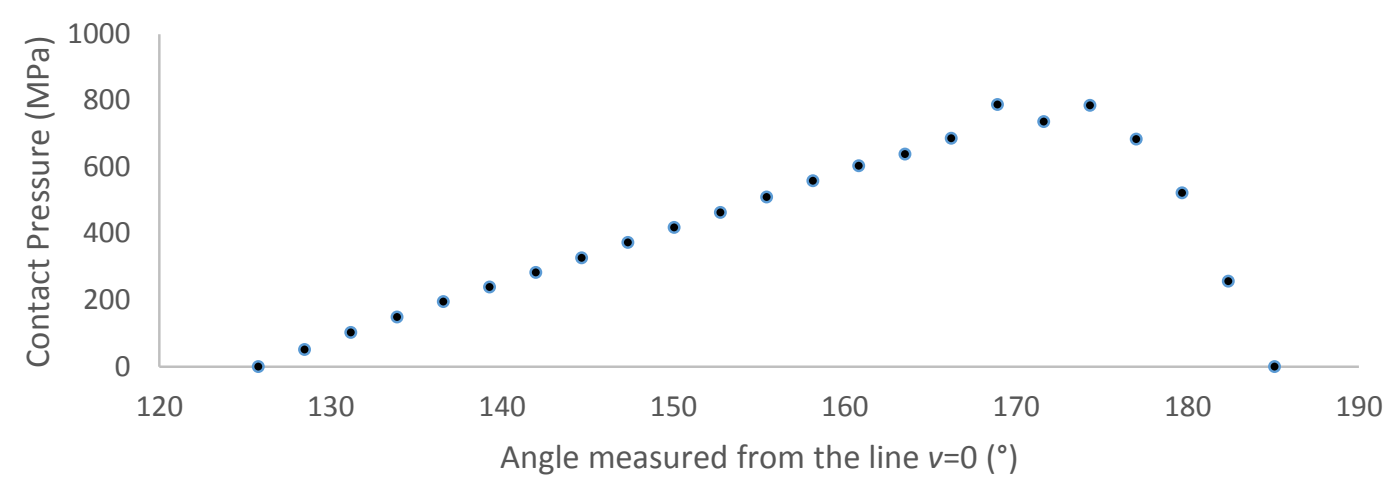

Figure 71: Contact Stress distribution for P3G transition fit for torsional load of $80 \mathrm{Nm}$ and bending load of $4600 \mathrm{~N}$

The shape of the contact stress distributions are skewed to the left as shown in Figures 70 and 71 for the clearance and transition fit and contact region is smaller in clearance fit. 


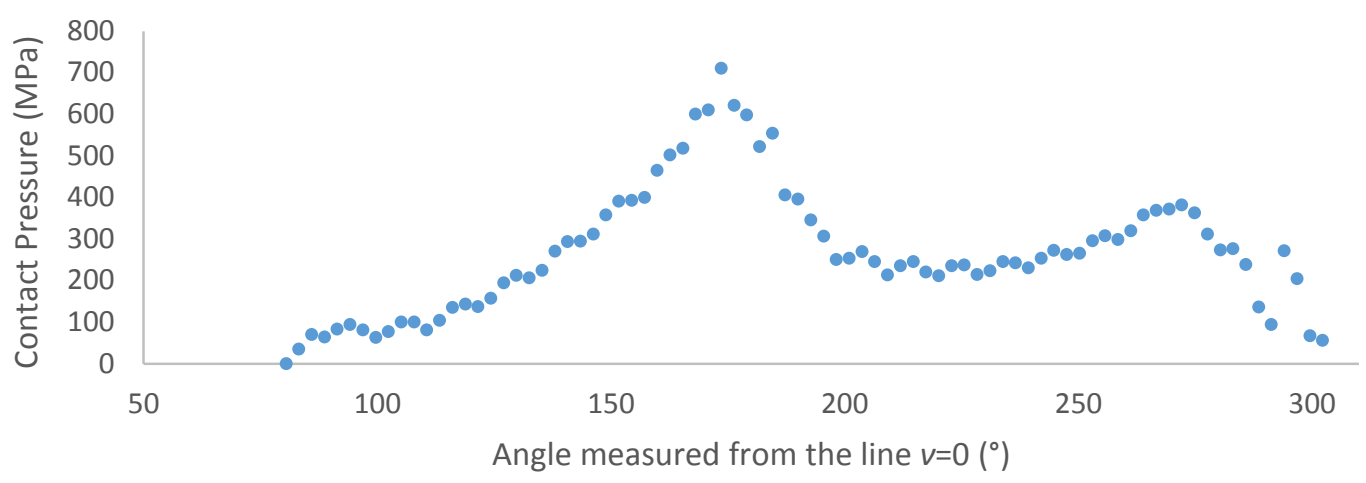

Figure 72: Contact Stress distribution for P3G interference fit for torsional load of $80 \mathrm{Nm}$ and bending load of $4600 \mathrm{~N}$

The interference fit has a crest in the middle, near $170^{\circ}$, unlike other fits who are skewed to left. It is due to the dominance of the bending load and its point of application relative to the contact zone.

The contact stress distribution for the P4C profiles are shown in Figures 73, 74, and 75.

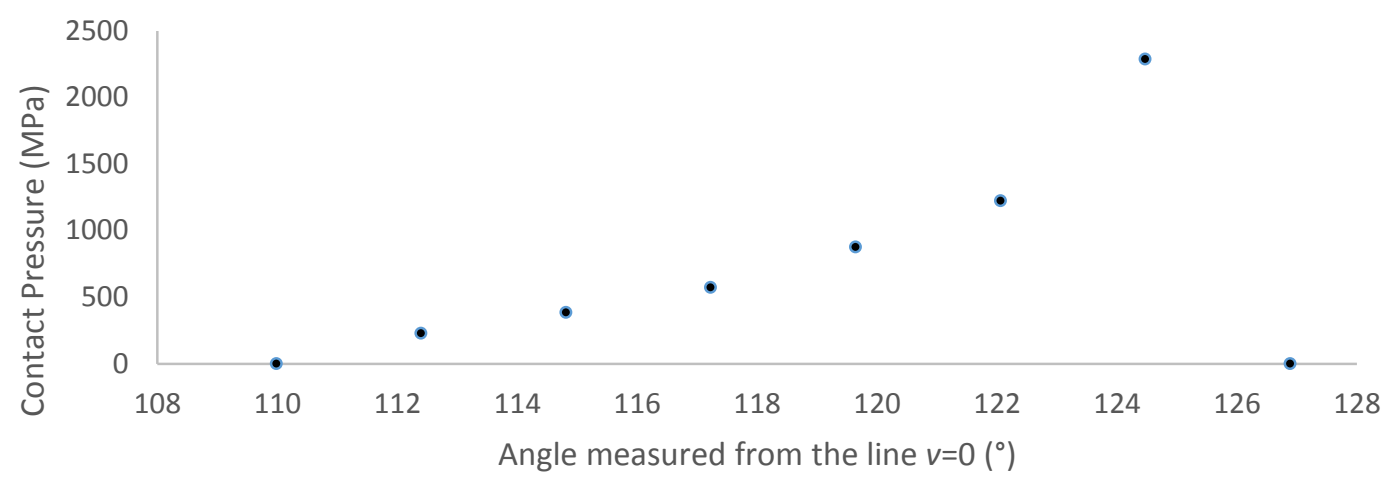

Figure 73: Contact Stress distribution for P4C clearance fit for torsional load of $80 \mathrm{Nm}$ and bending load of $4600 \mathrm{~N}$ 


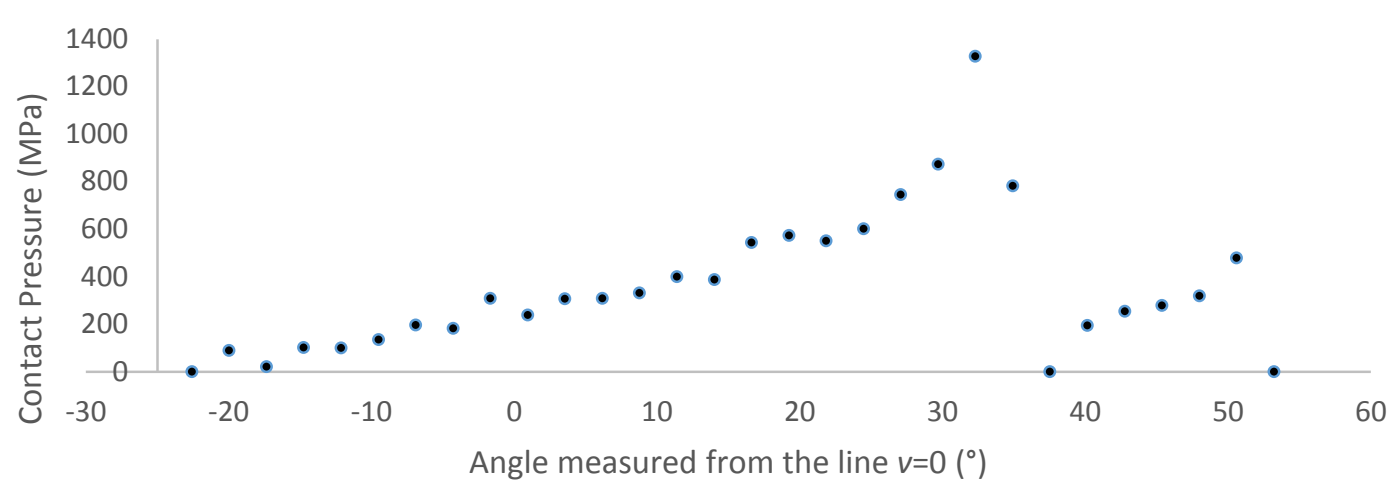

Figure 74: Contact Stress distribution for P4C transition fit for torsional load of $80 \mathrm{Nm}$ and bending load of $4600 \mathrm{~N}$

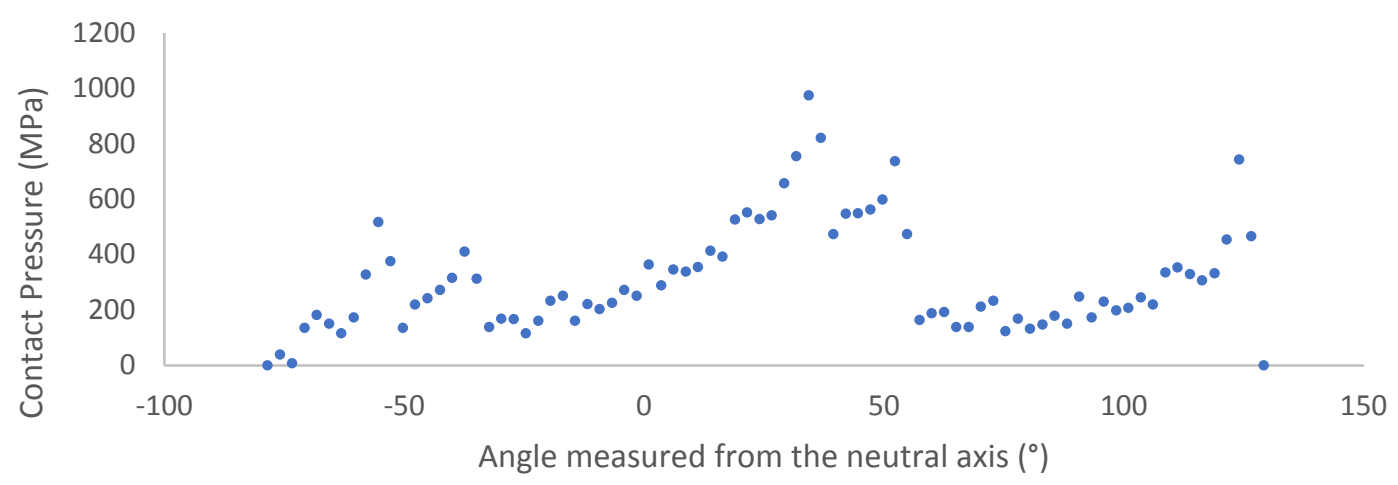

Figure 75: Contact Stress distribution for P4C interference fit for torsional load of $80 \mathrm{Nm}$ and bending load of $4600 \mathrm{~N}$

As seen from the Figures 73 and 74, the clearance and transition fit show similar shape and are skewed to the left. The value of contact stress is much higher in clearance fit in all cases. The interference fit in Figure 75 and transition fit in Figure 74 shows similar shape to the P3G interference fit with crest in the middle and then a sudden $\mathrm{t}$ in pressure followed by another small crest. The trough in the middle is due to expansion of the hub near the maximum contact region. For clearance fit, the shape of the contact stress can be approximated by a triangular shape. 


\subsection{5) Effect of loading on maximum von Mises stress in shaft}

The plots of contact stress and maximum von Mises stress in transient loading section showed that the von Mises stress distribution is similar to the contact stress distribution as contact stress is the major stress. However, some interesting results can be deduced about the location and magnitude of maximum von Mises stress by studying the variation on von Mises stress for each loading and fit. The effect on maximum von Mises stress on shaft has been presented for the three loading cases as follows.

i) Effect of pure torsional loading on maximum von Mises stress

The variation in the maximum von Mises stress for the connection subjected to pure torsional load of $80 \mathrm{Nm}$ is shown in Figure 76.

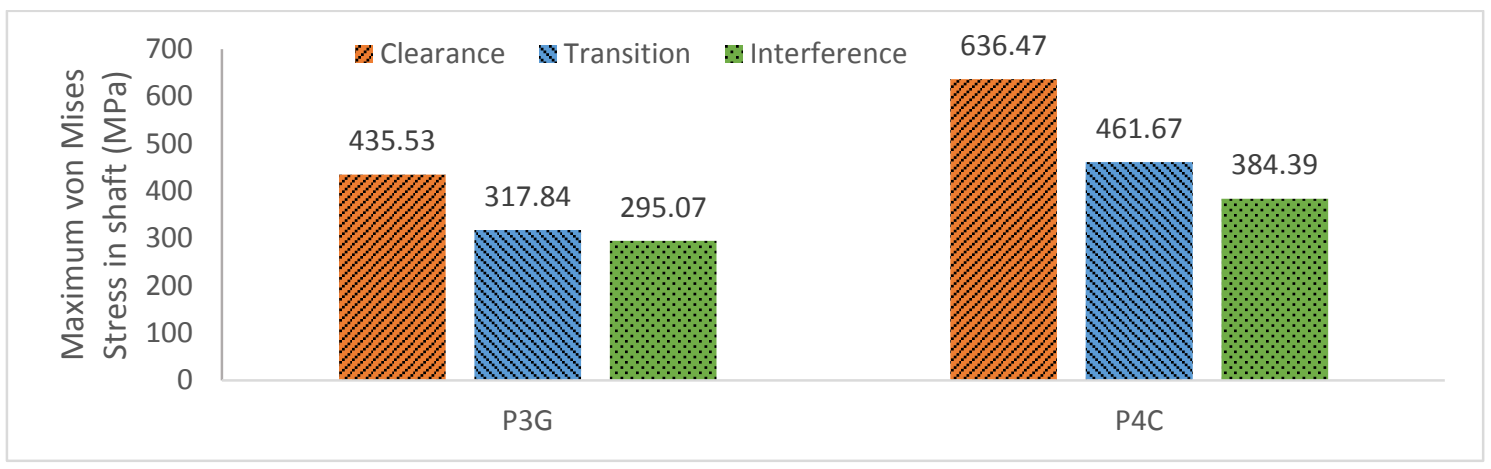

Figure 76: Maximum von Mises stress distribution for three and four lobe polygonal shaft subjected to pure torsional loading

The clearance fit has the highest von Mises stress compared to the other fits, due to a small contact area leading to greater contact pressure. The maximum von Mises stress is the lowest in P3G interference fit since it has larger contact area leading to smaller contact stress. The variation of stress between various fit is smaller in P3G shaft (38.45 \% between the clearance and interference fit) and greater in P4C shaft (49.39\% between the clearance and interference fit). The percentage difference between the two extreme cases, i.e. maximum von 
Mises stress between the clearance fit of P4C shaft and the interference fit in P3G shaft is 73.3 \%, suggesting P3G interference fit to be the most suitable connection for torsional load.

ii) Effect of torsional bending loading on maximum von Mises stress

Figures 77 and 78 show the effect on the von Mises stress on the shaft for torsional bending load case 1 and case 2 respectively. Comparing the two profiles, profiles, the P3G polygonal shaft has lower stress in all fits. The greatest stress is found in interference fit for loading case 1 followed by clearance fit. The higher stress in interference fit is due to the stress due from interference itself.

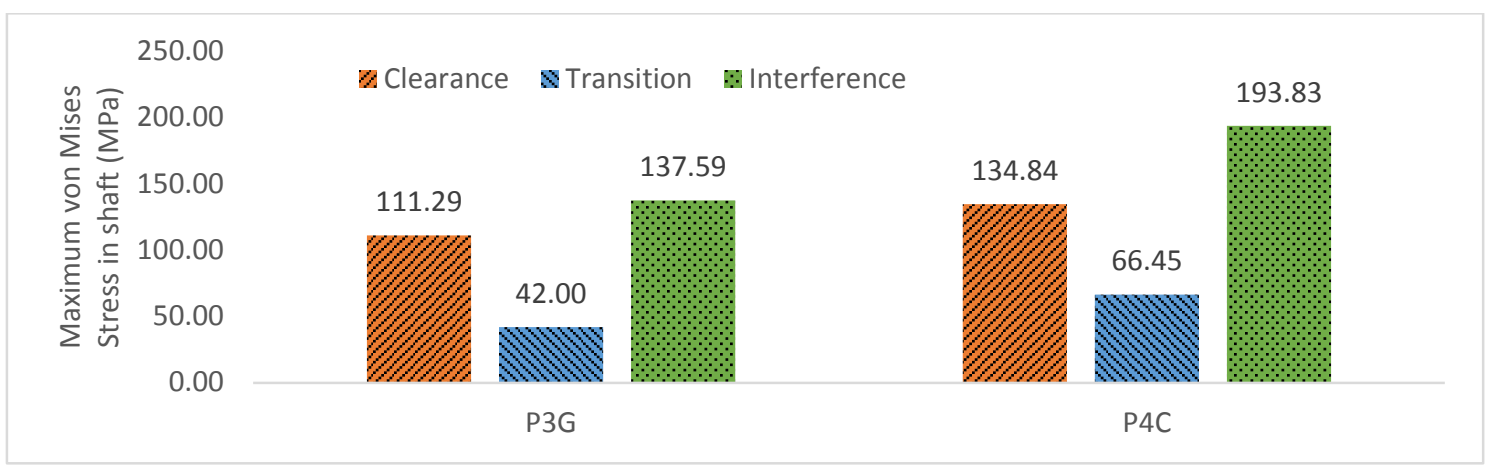

Figure 77: Maximum von Mises stress distribution for three and four lobe shaft subjected to torsional bending load 1

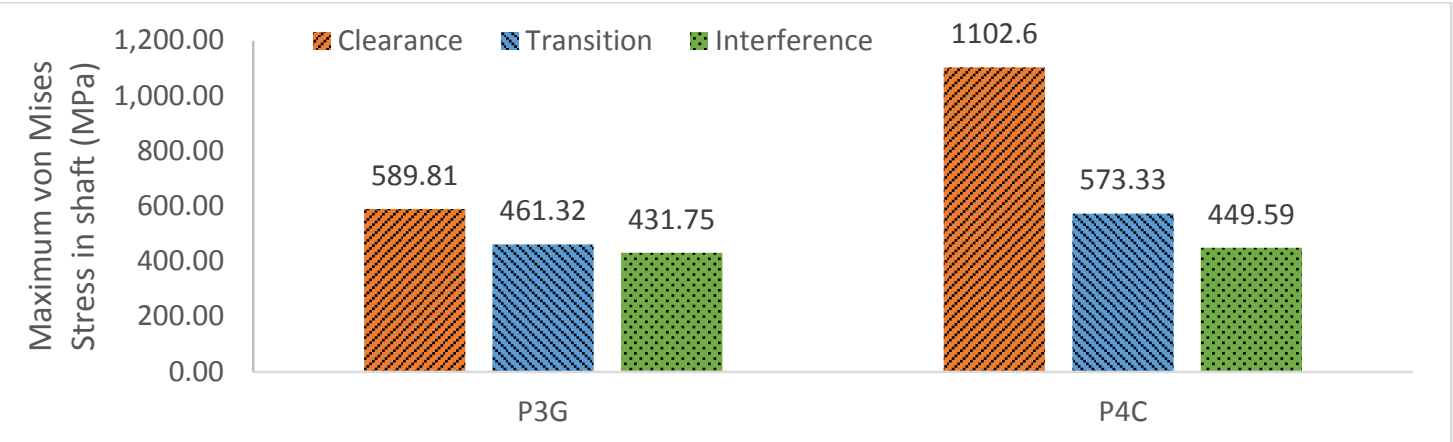

Figure 78: Maximum von Mises stress distribution for three and four lobe polygonal shaft subjected to torsional bending load 2 
For loading case 2, the greatest stress is found to be in clearance fit followed by the transition fit from Figure 78. The maximum von Mises stress was found to be the lowest in interference fit, due to larger conformal contact area and is supported by lower contact stress in Figure 64. The maximum von Mises stress was found to be greatest in P4C clearance fit. The small contact area in clearance fit is visible from the plot of clearance fit in Figure D4 and D12 in Appendix D. Unlike the transition or the interference fit, the shaft and the hub contact only at a smaller area in clearance fit. The P4C profile has a sharper edge due to discontinuity of the epitrochoidal curve and the circle, which digs in the hub, leading to much higher stress than the harmonious P3G profile. This discontinuity increases the chances of failure due to fretting fatigue in P4C clearance fit.

The maximum von Mises stress developed in the interference fit of P3G and P4C shafts are close to each other (26 \% and $4 \%$ difference in pure torsional and torsional bending load) and use of either shaft is acceptable for higher loading press fit applications. For other applications, P3G fit is preferred to P4C fit.

Figures 79 and 80 show the location of maximum von Mises stress on the cross section of the shaft.

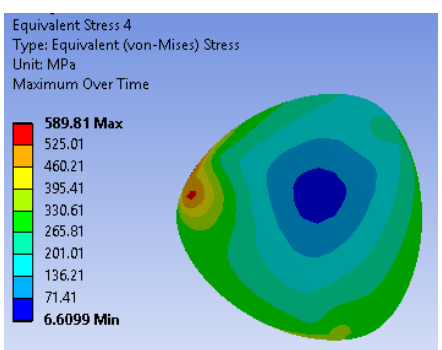

Clearance

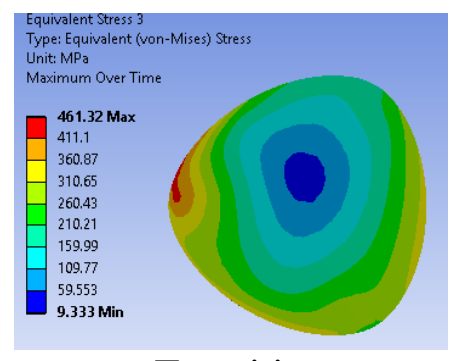

Transition

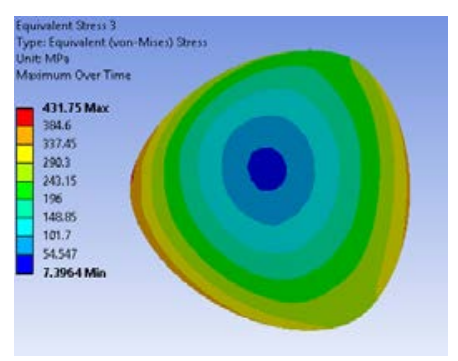

Interference

Figure 79: Position of maximum von Mises stress in P3G shaft for loading case 2 


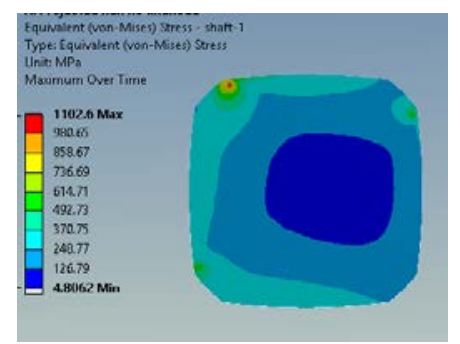

Clearance

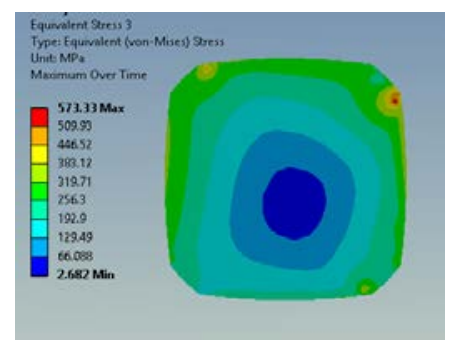

Transition

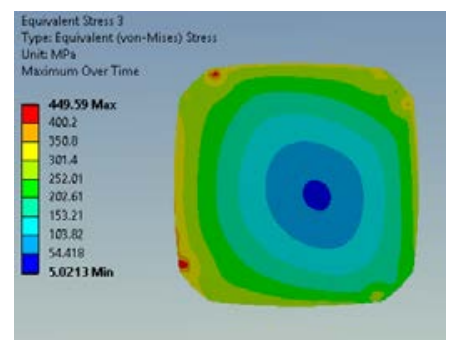

Interference

Figure 80: Position of maximum von Mises stress in P4C shaft for loading case 2

As evident from Figures 79 and 80, the maximum von Mises stress for clearance fit of both profiles in loading case 2 is below the surface causing the stress on the outer contact region to be lower. The larger applied load caused the hub to expand near the contact region and resulted in distinct regions of higher stresses, even in interference fit. For interference and transition fit, P3G shafts have maximum stress on the surface, while the P4C shafts have maximum stress below to the surface. In clearance fit, the contact region is smaller in width and can be approximated with a line contact between two cylinders. A line contact between two cylinders has maximum von Mises stress below the surface as a result of quadratic equation of curvature in Hertzian contact stress theory. This causes the maximum von Mises stress to be beneath the surface in clearance fit. As a result, the failure of the polygonal profile is likely to be from pitting and spalling below the outer edge of contact for clearance fit (Rossino, Castro, Moreto, Ruchert, Spinelli and Tarpani, 2014). The transition and interference fit of P3G shaft has larger contact area and cannot be approximated by line contact making the line fit assumption invalid.

\subsection{6) Effect of loading on maximum von Mises stress in hub}

The maximum von Mises stress for P3G and P4C hub at the three fits are shown in Figures 81, 82 and 83 for pure torsional load, torsional bending load case 1 and torsional bending load case 2 respectively. 


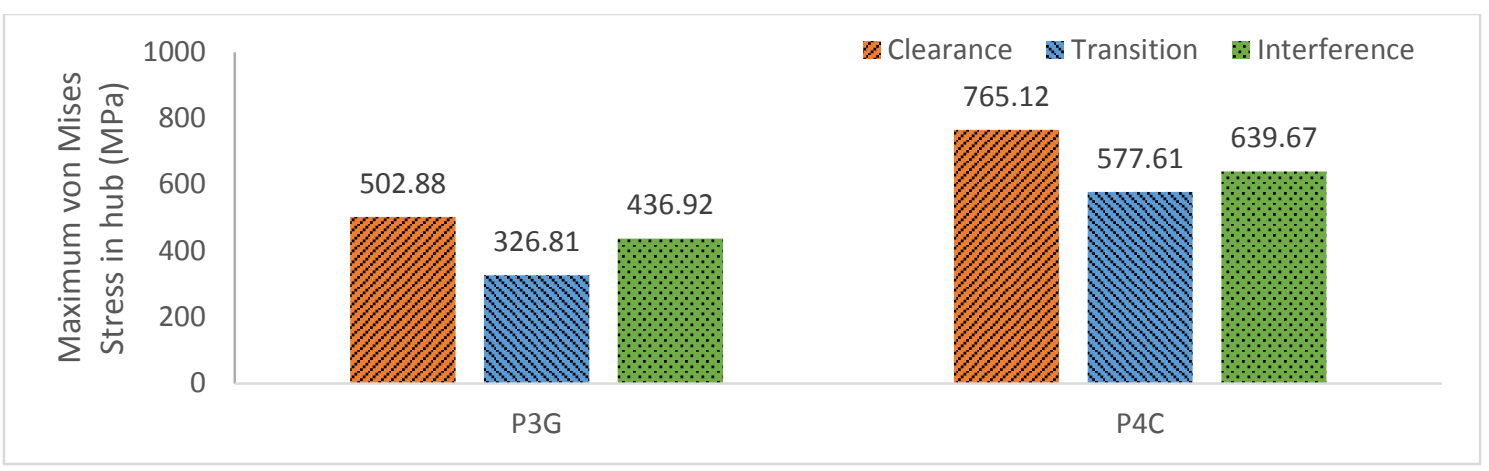

Figure 81: Maximum von Mises stress distribution for three and four lobe hub subjected to torsional load

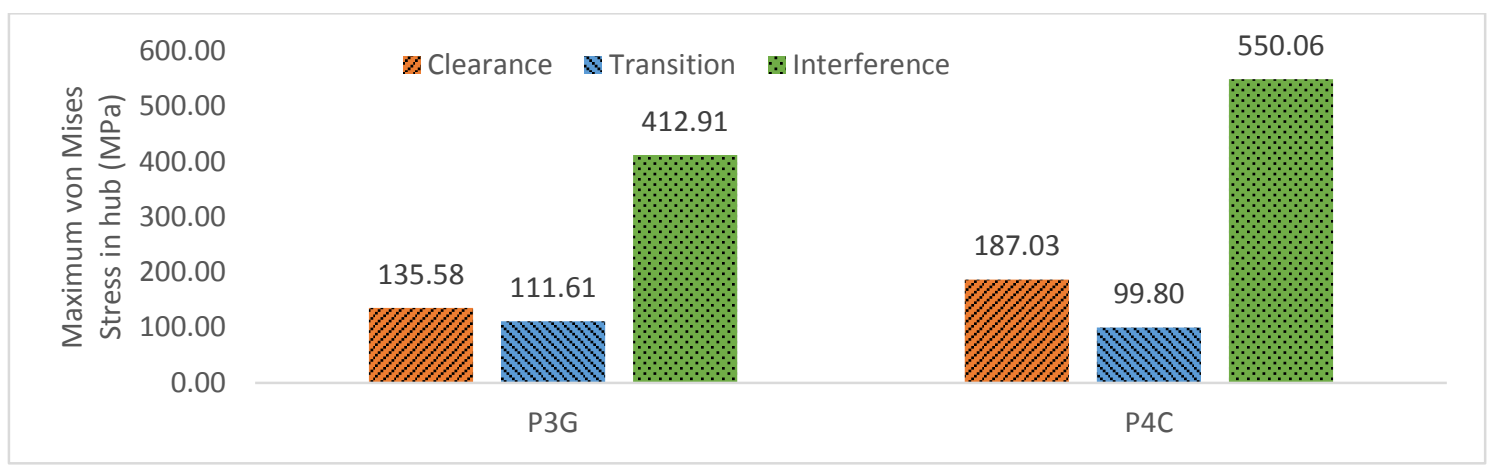

Figure 82: Maximum von Mises stress distribution for three and four lobe hub subjected to torsional bending load case 1

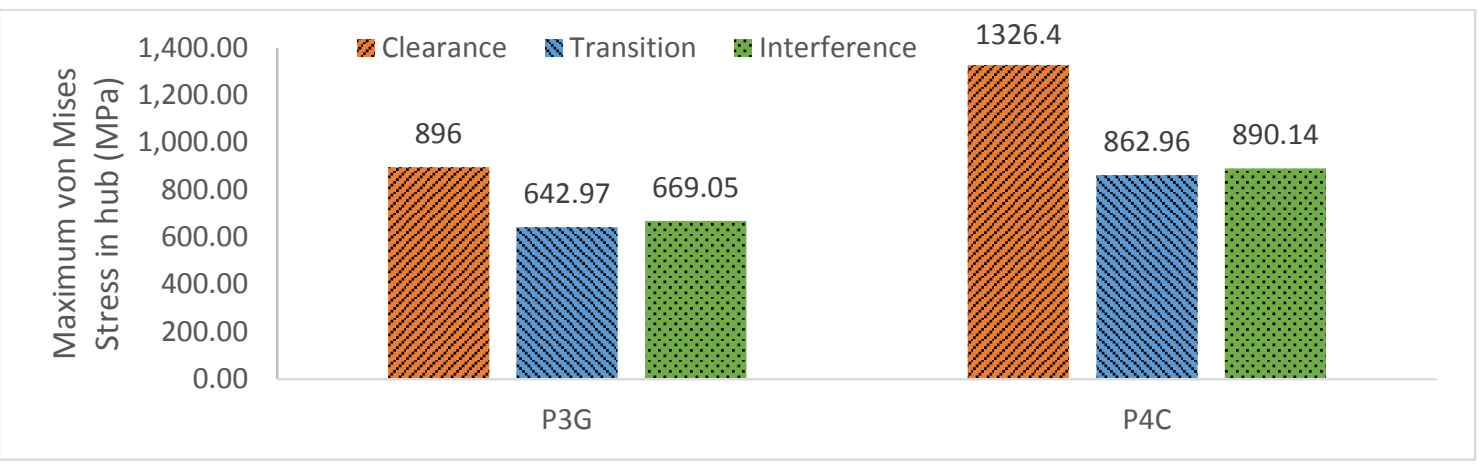

Figure 83: Maximum von Mises stress distribution for three and four lobe hub subjected to torsional bending load case 2

As evident from the figures, the trend is similar to the maximum von Mises stress in shaft. The stress in the P4C hub is more than the stress in P3G hub. This agrees with the results found by Winterfeld (2001), who found higher allowable stress in P3G profile than P4C profiles. 
The clearance fit is the most unsuitable fit for torsional or torsional bending load case 2. For torsional bending load case 1, i.e. when the load are small, the interference fit is the most vulnerable due to the rise of stress by interference itself. In loading case 2, the local plastic deformation occurs in the hub, but does not cause the structure to fail. However, it can act as a crack initiation site and cause failure by fretting as mentioned in Grossman (2007).

Hence, based on the results, the P3G shaft is recommended for higher torque transmission in interference fit. Although press fit application of P4C shaft can be used, the clearance fit should never be used for higher torque transmission. 


\section{4) Effect of friction}

Earlier studies by Czyzewski and Odman (1988) had neglected the effect of friction in the calculation of stress in the P3G polygonal shaft. Friction creates a resisting force to motion, that in turn decreases the overall stress experienced by the body. Hence, a realistic design of the polygonal shaft and hub should consider the effect of friction. Huang et al. (2010) did an in-plane stress analysis for torsional load and concluded that friction decreases the overall contact stress of the body, but has no other effect on the nature of the forces and its distribution in torsional loading. This section explains the effect of friction in torsional bending load as this represents a more realistic loading condition using FEA. The value of coefficient of friction $(\mu)$ has been considered as 0.18 from the studies of Grossman (2007). Models of P3G and P4C polygonal connections were subjected to torque of $80 \mathrm{Nm}$ and bending load of $4600 \mathrm{~N}$, as shown in Figure 84, with and without friction. The effect of friction has been explained in terms of variation in von Mises stress, contact pressure and hub displacement, with and without friction for a line to line fit of P3G and P4C shaft hub connection.

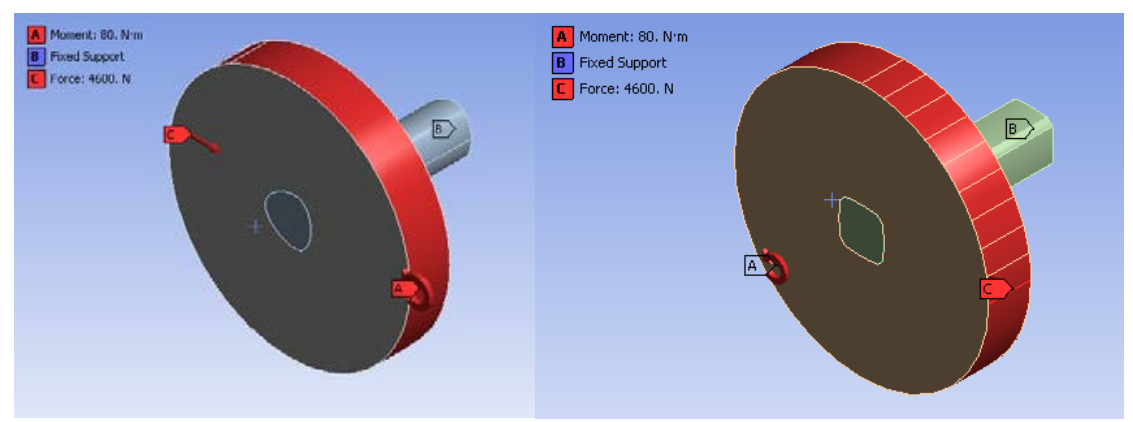

Figure 84: Loading used for determining effect of friction in P3G (left) and P4C (right) connection

\subsection{1) Effect of friction on von Mises Stress}

The von Mises stress distribution with and without friction in P3G hub is shown in Figure 85. The stress distribution pattern and position of maximum stress are found to be similar with 
and without friction. The magnitude of maximum stress is higher in the frictionless analysis. The highest stress on the frictionless hub is $44 \%$ higher than the highest stress on the frictional hub.
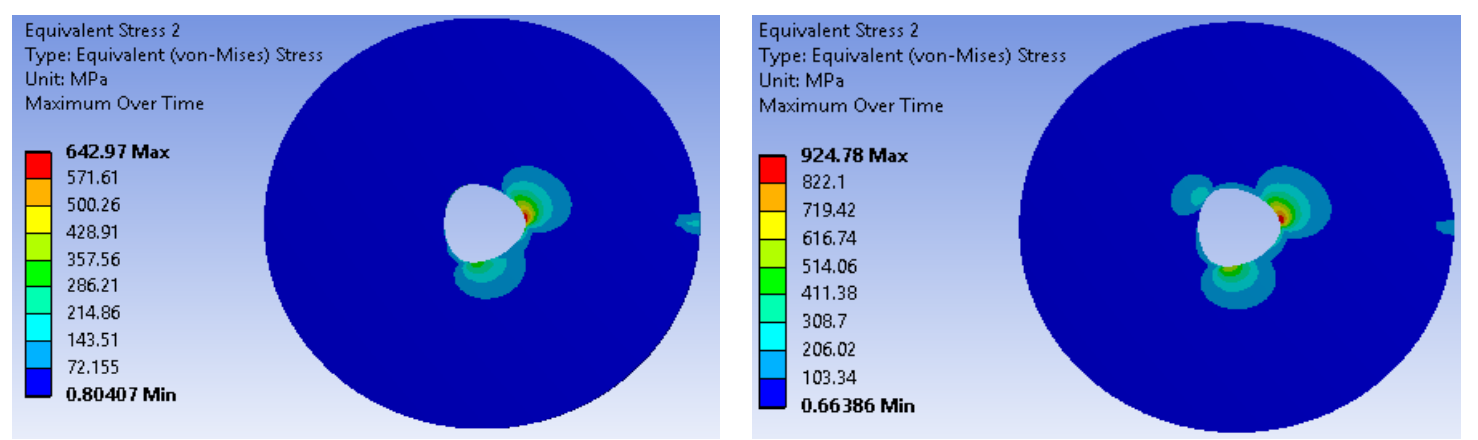

Figure 85: von Mises stress distribution in P3G hub with (left) and without (right) friction

Similar results can be found from the von Mises plot for P4C hub from Figure 86, where higher stress is found in the frictionless analysis. The maximum stress on P4C hub frictionless analysis is $28 \%$ higher than the maximum stress on frictional case.
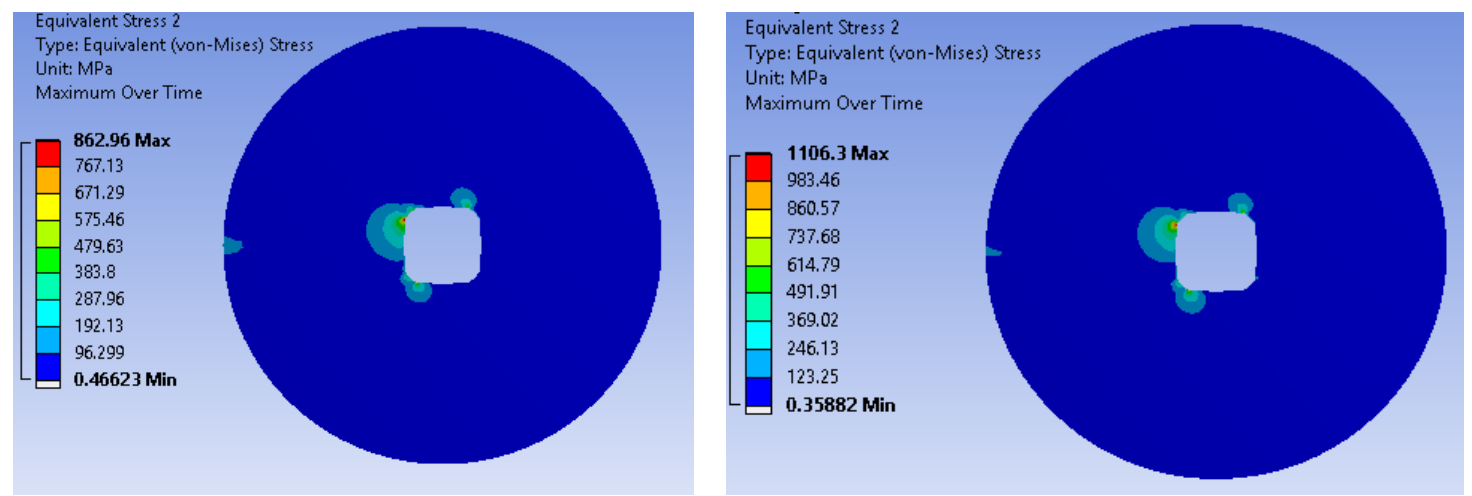

Figure 86: von Mises stress distribution in P4C hub with (left) and without (right) friction

Figures 87 and 88 show the von Mises stress distribution in the P3G and P4C shaft respectively. Although, the maximum stress is observed in the frictionless case as in the hub, the difference in their magnitude is not as drastic as in the hub. The lowered stress due to friction can be used in lowering the size of the material and cost for non-critical applications. The design of the connection without friction will overestimate the size and cost. 

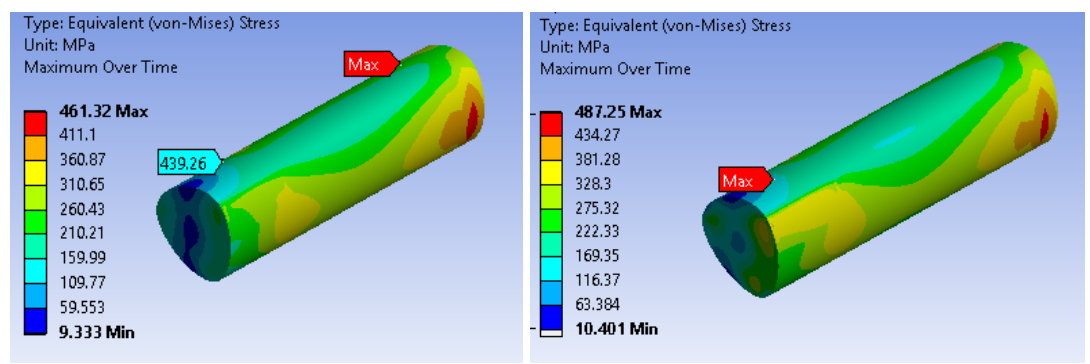

Figure 87: von Mises stress distribution in P3G shaft with (left) and without (right) friction
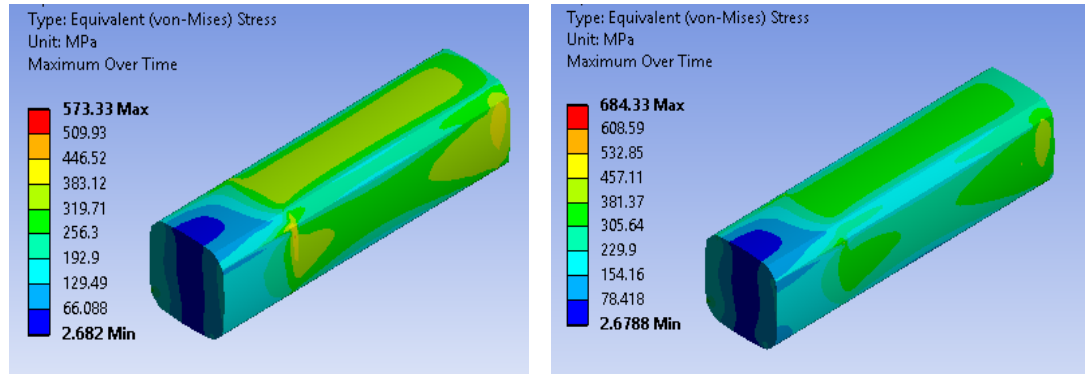

Figure 88: von Mises stress distribution in P4C shaft with (left) and without (right) friction

\subsection{2) Effect of friction on contact status}

The contact status is shown in Figure 89. As seen from the plot, the overall area of contact is similar with and without friction. On closer observation of the P3G connection, the non-frictional connection has a slightly larger sliding area than the frictional connection at the edge of connection. The larger contact area in the frictionless case is due to larger slippage of the connection in the absence of friction.
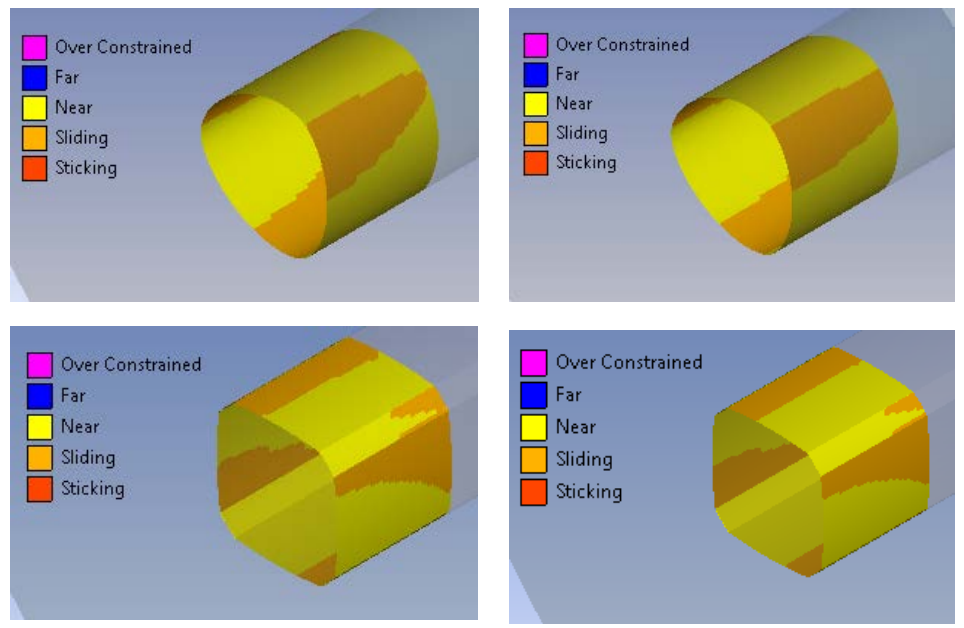

Figure 89: Contact status in P3G and P4C connection with (left) and without (right) friction. 


\subsection{3) Effect of friction on contact stress}

The contact stress plot for the P3G connection is shown in Figure 90. In frictionless connection, the contact slips till the interference from body stops it at the maximum pressure angle yielding higher normal stress due to curvature than the frictional case, where friction opposes slip at lower pressure angle. A similar result is obtained for P4C profile in Figure 91, with a similar stress pattern, differing in magnitude.
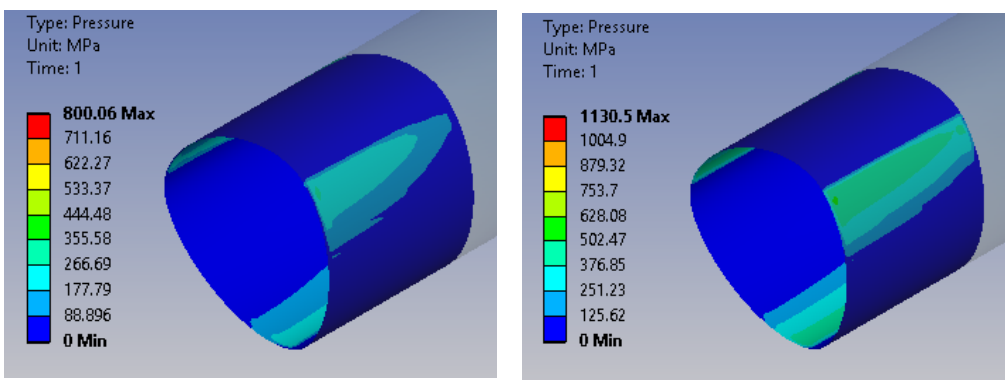

Figure 90: Contact Stress in P3G connection with (left) and without (right) friction
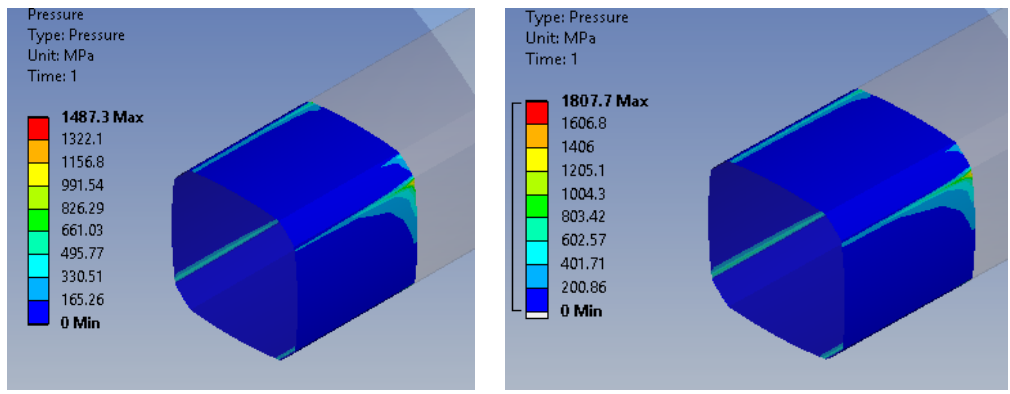

Figure 91: Contact stress in P4C connection with (left) and without (right) friction

\subsection{4) Effect of friction on torsional shear stress}

The friction only affects the contact stress values and does not change the stress values outside the contact region and the value of torsional shear stress and the distribution is similar, with and without friction. To demonstrate it, an element at the same location for P3G and P4C are chosen, with and without friction and shown in Figure 92. The element choses has the same node number for the eight nodes and the same location. Now, the value of the torsional shear stress is compared for the two elements. 
As seen from Figure 92, the P3G shaft has similar stress and the percentage difference with and without friction is $1.5 \%$ in P3G connection and $0.19 \%$ in P4C connection.

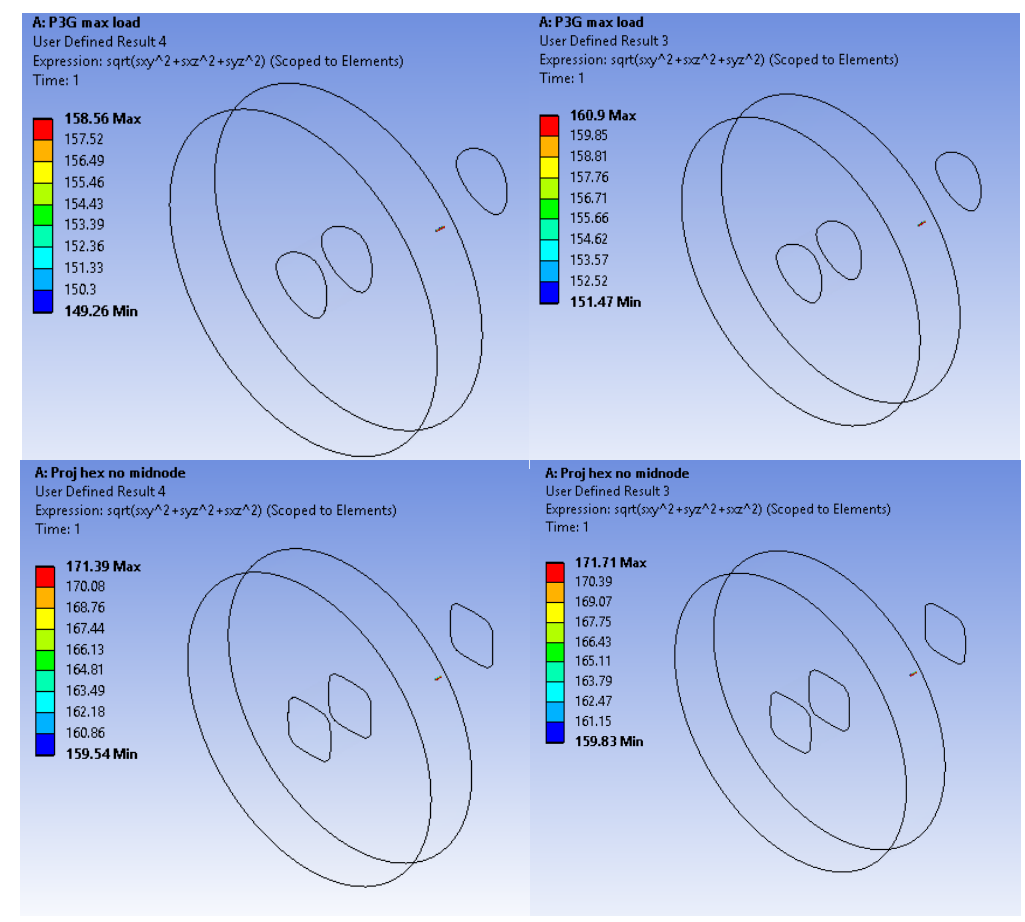

Figure 92: Resultant shear stress with (left) and without (right) friction

\subsection{5) Effect of friction on total displacements}

The total displacements in the axial direction are shown in Figure 93 for P3G hub and Figure 94 for P4C hub. It can be observed that the frictionless case has larger slip than the frictional case. This is obvious owing to the property of friction to oppose motion. The pattern of displacement vectors are the similar in both frictional and non-frictional cases.
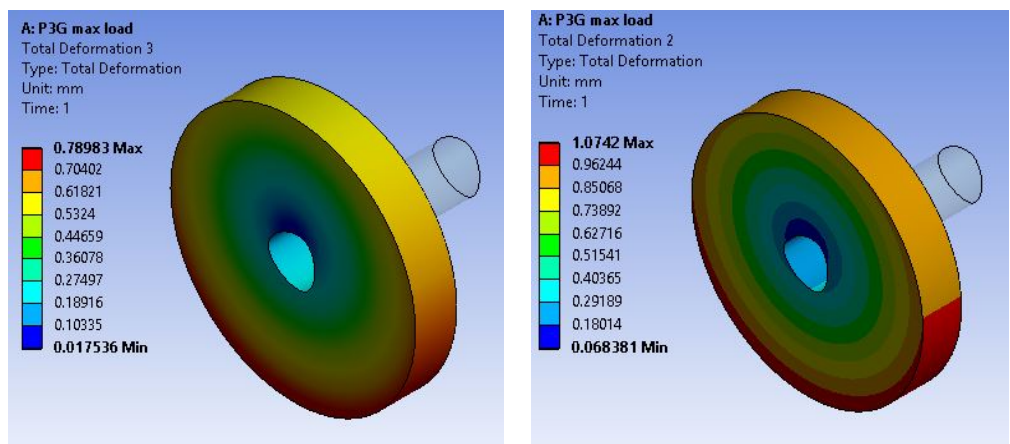

Figure 93: Total displacement (mm) in P3G connection with (left) and without (right) friction 

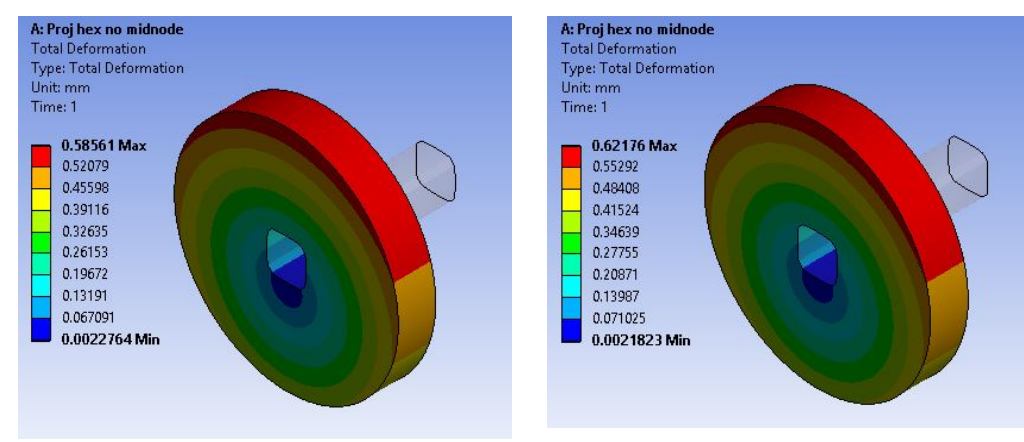

Figure 94: Total displacement (mm) in P4C connection with (left) and without (right) friction

4.4.6) Contact Stress distribution along the edge

The contact stress along the edge, where the hub and the shaft meet, is plotted in Figure 95 for P3G shaft and Figure 96 for P4C shaft. It can be observed that the shape of the curve is similar in both frictional and non-frictional cases. The only difference is in the value. It is worth noting that even in the case of line contact the pressure is concentrated only at a few points due to the expansion of the hub. The contact curve is triangular shaped in the P3G shaft and has a high crest and a trough followed by a smaller crest in the P4C shaft.
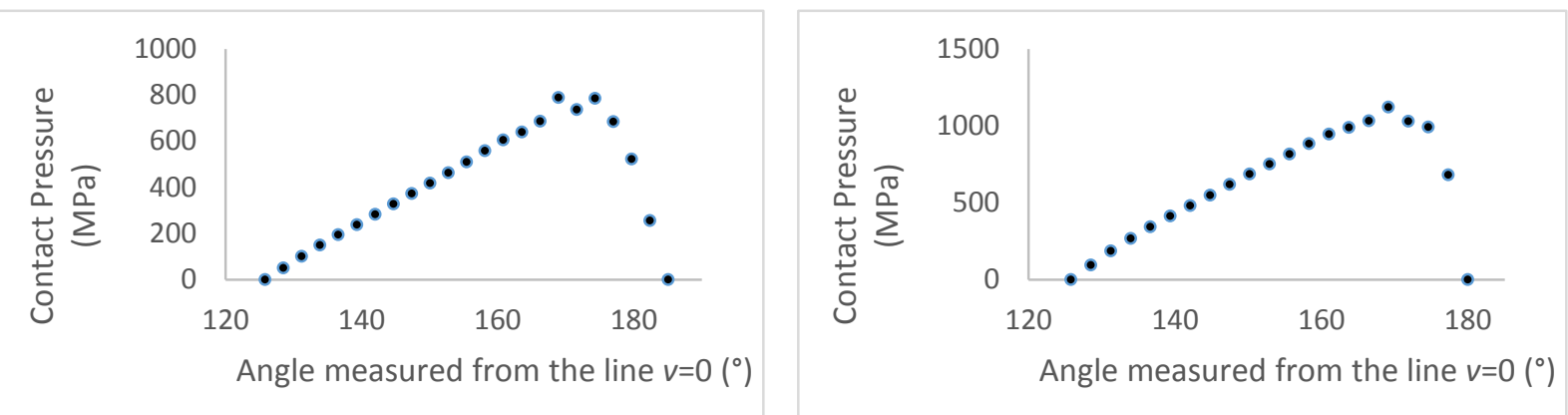

Figure 95: Contact stress variation in P3G shaft with (left) and without (right) friction
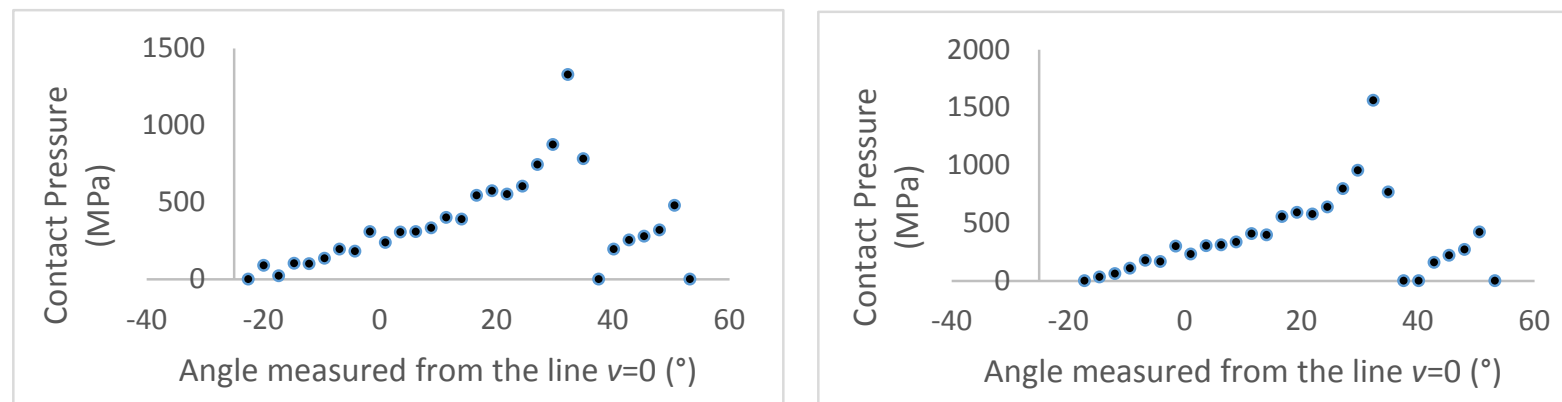

Figure 96: Contact stress variation in P4C shaft with (left) and without (right) friction 
In spite of the result showing friction to be beneficial for the connection, this is not always true because of the higher possibility of fretting fatigue as a result of friction. Although increasing the friction coefficient decreases the slip amplitude, it increases the frictional traction. Studies have shown that for a shaft hub connection, the increase in frictional traction is more detrimental than the decrease in slip amplitude and friction increases the possibility of frictional wear (Zehsaz \& Shahriary, 2013). This result is bolstered by the experimental results of Winterfeld (2001), who found a decrease in axial and von Mises stress due to an increase in coefficient of friction but a decrease in the torsional capacity of degreased surfaces as compared to an oil surface. The result of the friction analysis has been summarized in Table 11 .

Table 11: Summary of result from friction analysis

\begin{tabular}{|c|c|c|c|c|}
\hline \multirow{2}{*}{$\begin{array}{l}\text { Profile } \\
\text { Parameters }\end{array}$} & \multicolumn{2}{|c|}{ P3G } & \multicolumn{2}{|c|}{$\mathrm{P} 4 \mathrm{C}$} \\
\hline & Frictional & Frictionless & Frictional & Frictionless \\
\hline von Mises Stress in hub (MPa) & 642.97 & 924.78 & 862.96 & 106.30 \\
\hline von Mises Stress in shaft (MPa) & 461.32 & 487.25 & 573.33 & 684.33 \\
\hline Contact Status & \multicolumn{2}{|c|}{$\begin{array}{l}\text { almost same status but } \\
\text { frictionless has a slightly } \\
\text { more sliding area near edge }\end{array}$} & \multicolumn{2}{|c|}{ similar sliding } \\
\hline Contact Stress (MPa) & 800.06 & 1130.50 & 1487.30 & 1807.70 \\
\hline Total Displacement in hub & \multicolumn{4}{|c|}{ Slightly greater in frictionless case } \\
\hline Contact Stress along edge & & ape & ig only & itude \\
\hline
\end{tabular}




\section{Chapter 5: Conclusion}

With a strong conformance to the analytical, DIN standard, and the experimental results, the finite element analysis was able to predict the behavior of polygonal shaft and hub in torsional bending load. For both pure torsional and torsional bending load, the hub developed higher stress than the shaft. The friction was found to lower the stress value, although it did not change the stress distribution.

For a pure torsional load, the clearance fit developed the highest stress for both three and four lobe profiles, due to a smaller contact region. For a fit, the contact area was smaller in the P4C connection compared to the P3G connection. The area of contact for the P4C shaft was near the discontinuity of the curve and the higher pressure angle was responsible for smaller contact area and consequently higher contact stress in the P4C profile. The clearance fit had the lowest normal axial stress and the value was smaller in P4C profile compared to the P3G profile. This makes P4C an ideal choice for sliding fit applications. For normal power transmission, a P3G shaft with interference or transition fit is recommended over P4C shaft for a constant grinding diameter subjected to a pure torsional load applications.

For a torsional bending load, the P3G shaft developed lower stress compared to the P4C shaft, based on maximum von Mises stress. For an interference fit, the difference between the maximum von Mises stress of the two shafts was not as significant as compared to the difference in clearance fit (4.06 \% difference in interference fit and $60.6 \%$ difference in clearance fit and $21.65 \%$ in transition fit for maximum von Mises stress). This infers suitability of either profile in interference fit and unsuitability of P4C profile in clearance fit. The contact stress distribution was found to be triangular and skewed to one side for clearance and transition fits. The contact stress had a higher crest at one side, trough in the middle and a small crest on other side across the contact zone for interference fit for both profiles. For both profiles, the interference fit 
developed the least stress for higher power transmission (24.19\% and $84.14 \%$ lower in P4C interference fit compared to transition and clearance fit respectively). The normal stress along the axis was found to be lower in P4C shaft in clearance fit (53.21 \% lower than P3G clearance), making it a preferred choice in sliding fit application for lower stresses. For large power transmissions, the interference fit is recommended and clearance fit is found to be unsuitable. The possibility of pitting was found to be higher, especially in P4C, due to the location of maximum von Mises stress beneath the surface.

The next step in the analysis of polygonal shafts could be a fretting fatigue test, using shaft and hub subjected to a similar load on different fits. It is also recommended to make a U.S. standard for polygonal profile design using the contact stress values for various fits. 
Appendices 
Appendix A: Dimension of the shaft and hub used in Experiment

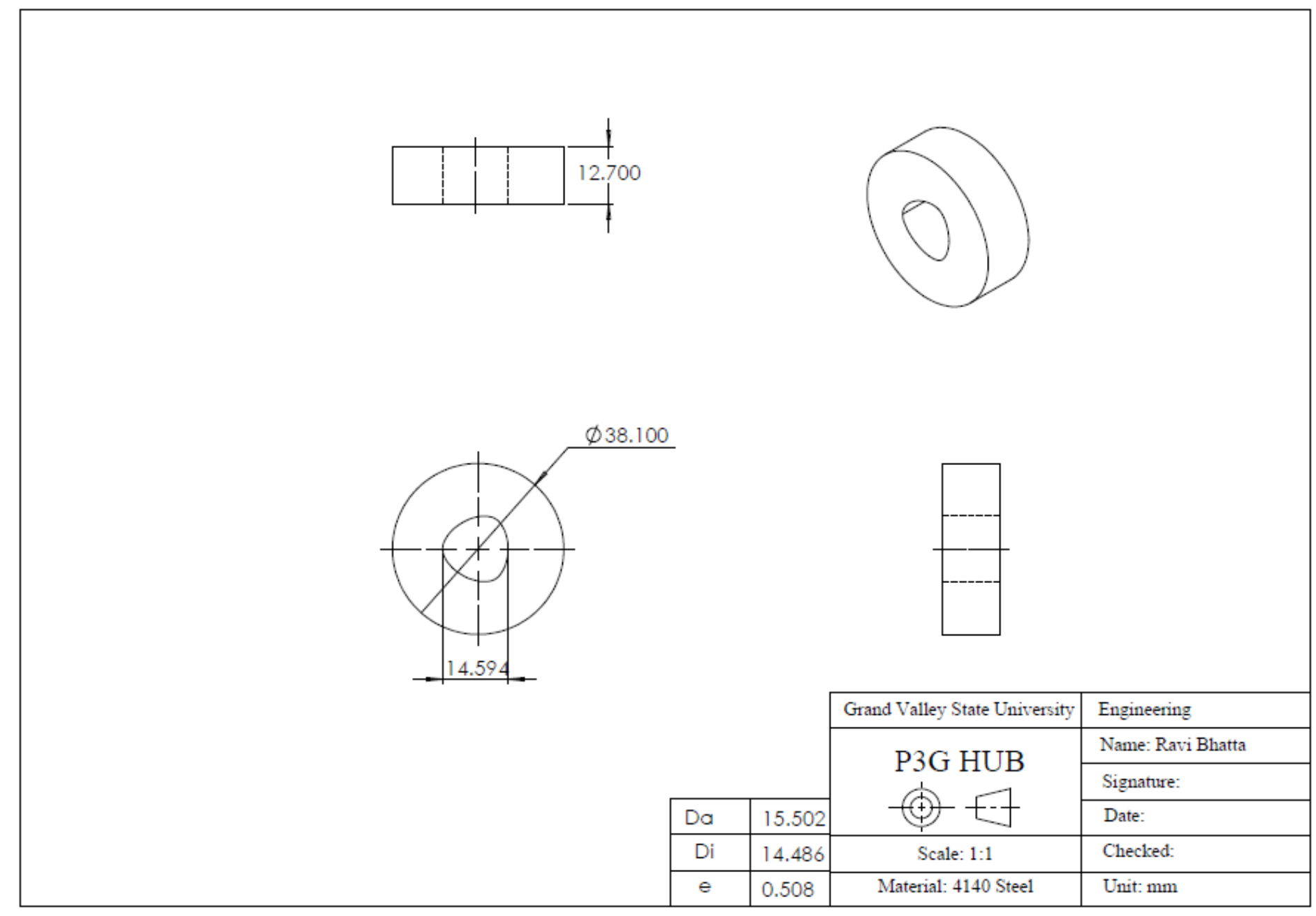




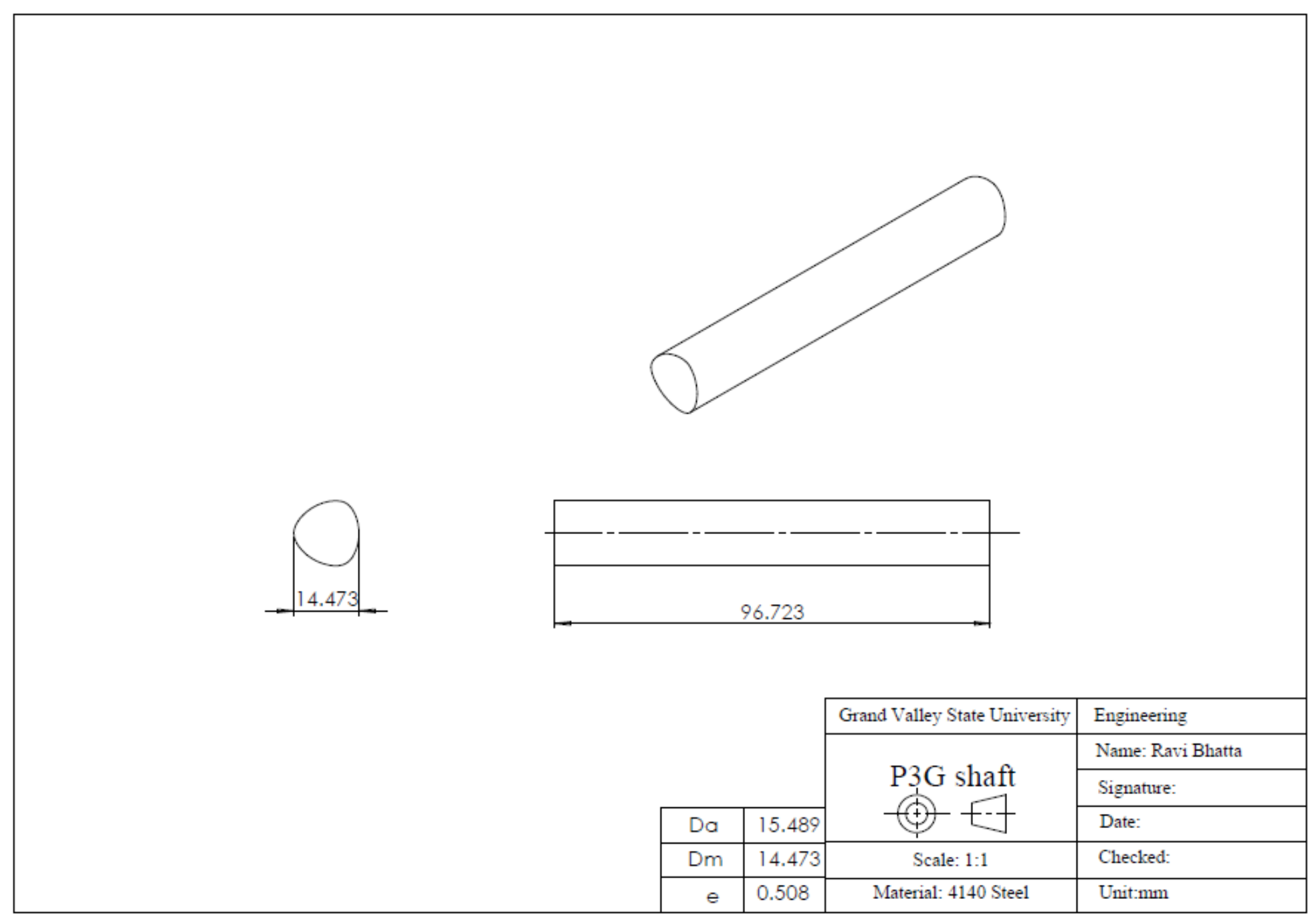




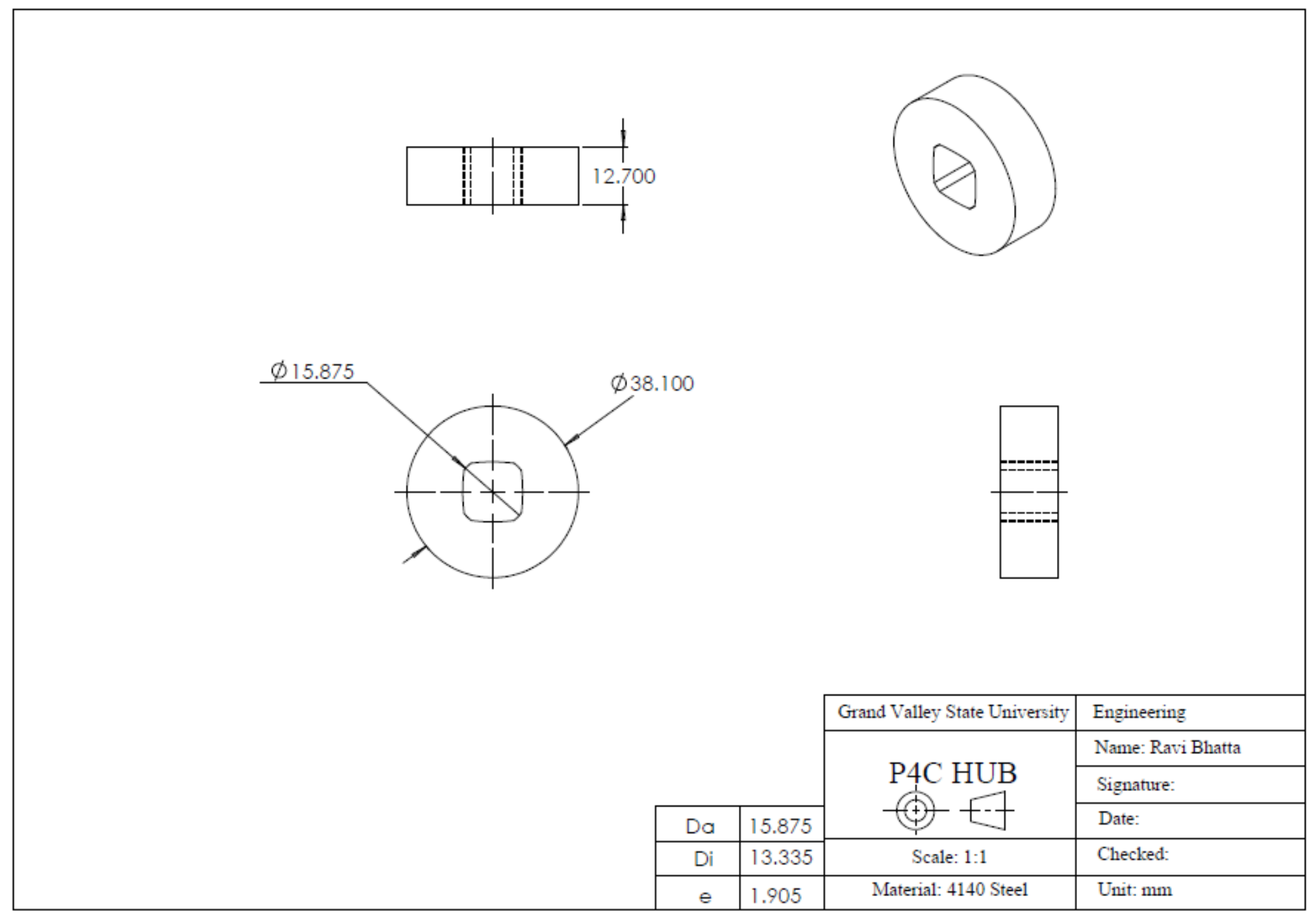




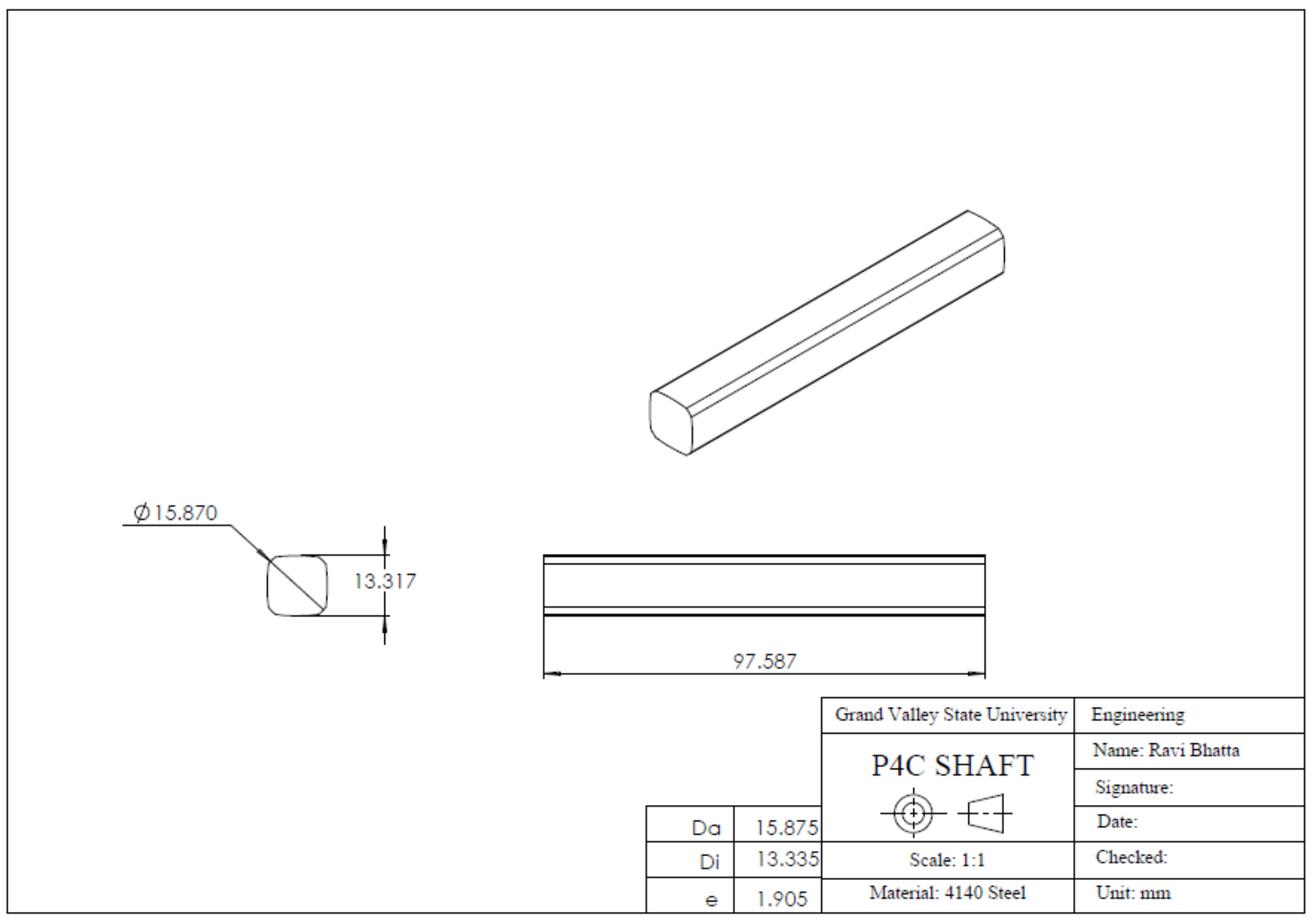




\section{Appendix B: Load Calculation for FEA}

\section{Load Calculation for FEA.}

For performing the finite element analysis, the magnitude of bending and torsional load needs to be determined. For determining the load, an approximate load calculation was performed for P4C polygonal shaft and the same value was applied to the P3G connection. The loading was supposed to be torsional bending load from a spur gear connection with pressure angle of $20^{\circ}$. The cross section of the P4C shaft used for the calculation is shown in Figure B1.

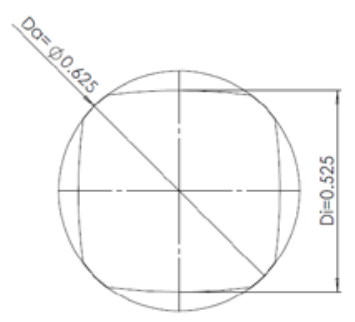

Figure B1: Cross section of P4C shaft

Grinding Diameter of shaft $\left(\mathrm{Da}=\mathrm{d}_{1}\right)=15.875 \mathrm{~mm}$

Internal Diameter of shaft $\left(\mathrm{Di}=\mathrm{d}_{2}\right)=13.335 \mathrm{~mm}$

Eccentricity of the shaft (e): $1.905 \mathrm{~mm}$

Nominal Diameter $\left(\mathrm{D}_{\mathrm{m}}\right)=\mathrm{D}_{\mathrm{i}}+2 \mathrm{e}=13.335+2 \times 1.905=17.145 \mathrm{~mm}$

Length of shaft $(\mathrm{L})=101.6 \mathrm{~mm}$

Width of hub $(l)=25.4 \mathrm{~mm}$

The loading is combined torsion and bending, as shown in Figure B2, where the end forces are reaction forces. 


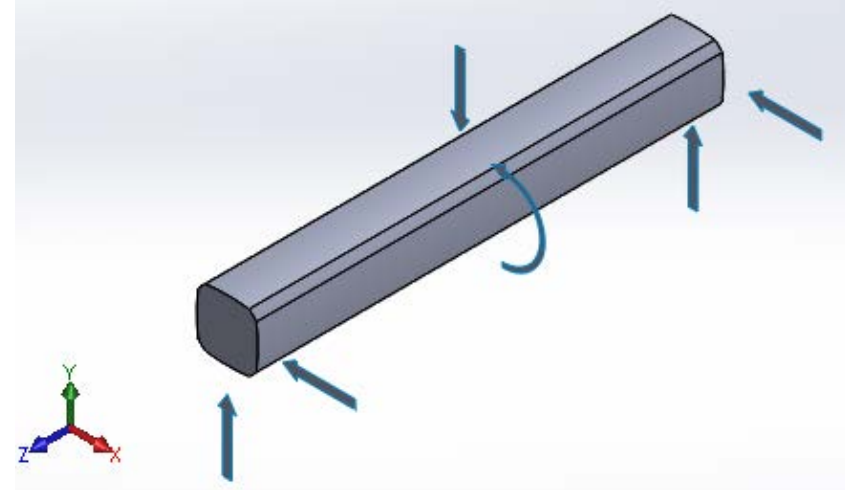

Figure B2: Loading for finite element analysis

A stress element on the upper left portion of the shaft, where the shaft and the hub meet, is considered. The element is subjected to the normal force in axial direction $\left(\sigma_{\mathrm{z}}\right)$, torsional shear stress and contact stress. The torsional shear is the same in all four contact regions, but the bending stress is maximum in the upper left element among all four regions. This upper left element is the critical part for analysis. The combined effect of the three forces can be used to calculate the approximate loading capacity of the system using distortion energy theorem.

For the torsional load, considering the same small element, the torsion is created by shear stresses acting on the $\mathrm{x}$ plane in $\mathrm{z}$ direction $\left(\tau_{\mathrm{xz}}\right)$. For the stress element, neglecting the stresses due to warpage,

$$
\sigma_{\mathrm{x}}=\sigma_{\mathrm{y}}=0 \text { and } \tau_{\mathrm{xy}}=\tau_{\mathrm{yz}}=0
$$

where, $\sigma_{\mathrm{i}}$ is the normal stress in $\mathrm{i}^{\text {th }}$ direction and $\tau_{\mathrm{xy}}$

Let $F_{r}$ and $F_{t}$ be the radial and tangential force from the gear The maximum bending moment for a beam of length L, fixed at both ends and subjected to a point load $\mathrm{P}$ at the midpoint is given as: 


$$
\text { Maximum Bending Moment(M) }=\frac{F_{r} L}{8}
$$

The bending stress is given as:

$$
\sigma_{z}=\frac{\mathrm{My}}{\mathrm{I}}
$$

where, $\sigma_{z}$ is the normal stress in axial direction, $\mathrm{M}$ is the bending moment, $\mathrm{y}$ is the distance from the neutral axis and I is the moment of Inertia about the neutral axis. For the given problem, the ends of the shaft were initially constrained in $\mathrm{x}$ and $\mathrm{y}$ direction, but the rigid body motion occurred in the simulation requiring the constraining of the shaft from all motion. The maximum bending stress in a shaft constraint at both ends with a load of $F_{r}$ in the middle of $L$ length is given as:

$$
\begin{gathered}
\sigma_{z}=\frac{\frac{F_{r} L}{8} \frac{d_{2}}{2}}{I} \\
\sigma_{z}=\frac{\frac{F_{r} 101.6}{8}\left(\frac{13.335}{2}\right)}{2140.9} \\
\sigma_{z}=0.03955 F_{r}
\end{gathered}
$$

Considering the power transmission from a spur gear, pressure angle for a typical spur gear power transmission is considered as $20^{\circ}$. The frictional force is given by the pressure angle of the polygonal shaft, maximum of $29^{\circ}$ for given geometry. Assuming an ideal point contact due to expansion of hub for force calculation, the pressure angle at the tip of the epicycloidal curve and the circle meet is around $29^{\circ}$. The forces are:

Radial force due to bending: $\quad F_{r}=F_{t} \tan \left(20^{\circ}\right)$

Normal force due to contact: $\quad F_{n}=F_{t} \sin \left(29^{\circ}\right)$

Frictional force: $\quad F_{f}=F_{t} \cos \left(29^{\circ}\right)$ 
The normal stress reduces to:

$$
\sigma_{z}=0.03955 F_{t} \tan 20^{\circ}=0.0144 F_{t}
$$

Also, from DIN standards, maximum shear stress is given as:

$$
\begin{gathered}
\tau_{x z}=\frac{T}{W p}=\frac{T}{0.2 d_{2}{ }^{3}} \\
\tau_{x z}=\frac{F_{f} d_{1}}{0.4 \times\left(d_{2}\right)^{3}} \\
\tau_{x z}=0.0167 F_{f} \\
\tau_{x z}=0.0167 F_{t} \cos 29^{\circ} \\
\tau_{x z}=0.0146 F_{t}
\end{gathered}
$$

Although, the maximum shear stress is not the same for the point of contact, this stress has been considered to be conservative on the maximum load application and lack of actual stress distribution.

The contact stress value is given by the DIN standard for only torsional loading as:

$$
\begin{gathered}
\mathrm{P}=\frac{\mathrm{T}_{\max }}{\mathrm{l}\left(\pi \mathrm{e}_{r} \mathrm{D}_{\mathrm{r}}+\frac{\mathrm{D}_{\mathrm{r}}^{2}}{20}\right)} \\
\mathrm{P}=\frac{F_{f} \frac{d_{1}}{2}}{\mathrm{l}\left(\pi \mathrm{e}_{r} \mathrm{D}_{\mathrm{r}}+\frac{\mathrm{D}_{\mathrm{r}}^{2}}{20}\right)} \\
\mathrm{P}=0.0064 F_{f} \\
\mathrm{P}=0.0064 F_{t} \cos 29^{\circ} \\
\mathrm{P}=0.0056 F_{t}
\end{gathered}
$$


The contact pressure due to the radial force acting on the conformal convex concave surface has not been considered here, which will increase the total contact pressure and can be compensated by taking load with high factor of safety.

The three stresses (normal, contact, and shear) are mutually perpendicular to each other, so the combined effect of these forces can be evaluated using the von Mises stress for 4140 steel (Yield stress of $965 \mathrm{MPa}$ of oil quenched 4140 taken for this simulation purpose only) as:

$$
965=\sqrt{\left(0.0144 F_{t}\right)^{2}+\left(0.0056 F_{t}\right)^{2}-\left(0.0144 F_{t}\right) 0.0056 F_{t}+3\left(0.0146 F_{t}\right)^{2}}
$$

For factor of safety of 30 so that the yield stress is not exceeded at any point, the forces are:

$$
\begin{gathered}
\mathrm{F}_{\mathrm{t}}=1139.00 \mathrm{~N} \\
F_{r}=F_{t} \tan 20^{\circ} \\
F_{r}=414.56 \mathrm{~N} \approx 410 \mathrm{~N}
\end{gathered}
$$

Torque on shaft:

$$
\begin{gathered}
T=F_{f} \times \frac{d_{1}}{2} \\
\mathrm{~T}=7.91 \mathrm{Nm} \approx 8 \mathrm{Nm}
\end{gathered}
$$




\section{Appendix C: Experimental Stress Determination}

\section{Experimental Stress Determination: von Mises stress calculation from Strain Gage}

For the loading as showing in Figure C1, considering a stress element on the top middle portion of the cross section of the shaft at a mid-distance from the support and the hub, the stresses in the element are the axial stress due to the bending load and the shear stress due to torsion. The other stresses are zero on the outer face and the condition of plane stress can be realized.

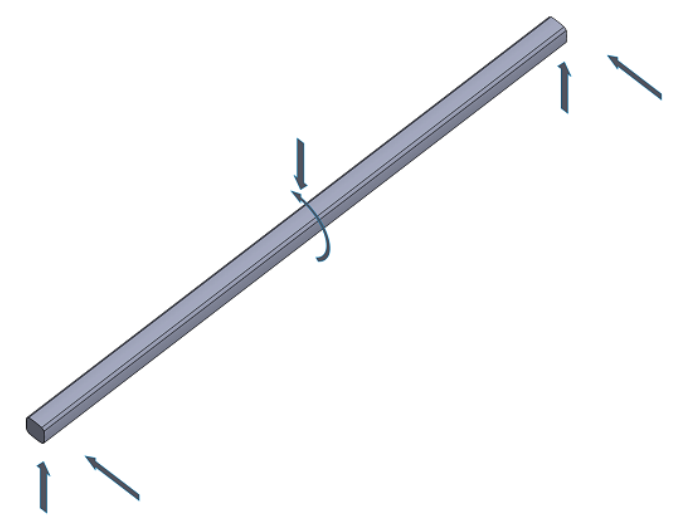

Figure C1: Schematic of experimental loading

For measuring the stress accurately, the strain needs to be measured at the same point using a strain rosette. The plain rectangular strain rosette could be an option to find the stress in the element. The stacked strain rosette was selected for the experiment, owing to the difference in the stress in different position of the three strain gage. The rosette strain gage of $0.787 \mathrm{~mm}$ width for each was used to measure strain.

For calculation of the von Mises stress at a particular location using strain rosette, the rosette strain gage was aligned in a way so that the middle gage was parallel to the $\mathrm{Z}$ axis, according to Figure C2. For the strain gage at an angle of $\theta$ to the $\mathrm{Z}$ axis, the measurement from a strain gage $\varepsilon$ is given as: 


$$
\varepsilon=\varepsilon_{\mathrm{z}}(\cos \theta)^{2}+\varepsilon_{\mathrm{y}}(\sin \theta)^{2}+\gamma_{\mathrm{zy}} \sin \theta \cos \theta
$$

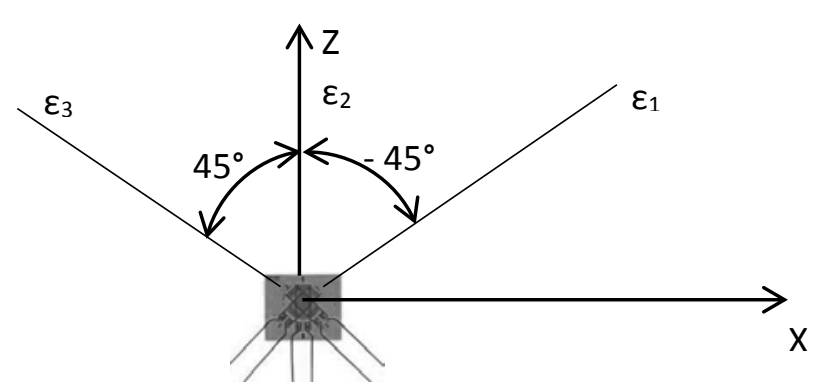

Figure C2: Orientation for finding the von Mises stress

Referring to the orientation shown in Figure C2:

$\theta_{1}=-45^{\circ}$ for $\varepsilon_{1}, \theta_{2}=0^{\circ}$ for $\varepsilon_{2}, \theta_{3}=45^{\circ}$ for $\varepsilon_{3}$

The equation (C1) reduces to:

$$
\left.\begin{array}{c}
\varepsilon_{1}=\varepsilon_{\mathrm{z}}\left(\cos \left(-45^{\circ}\right)\right)^{2}+\varepsilon_{\mathrm{x}}\left(\sin \left(-45^{\circ}\right)\right)^{2}+\gamma_{\mathrm{zx}} \sin \left(-45^{\circ}\right) \cos \left(-45^{\circ}\right) \\
\varepsilon_{2}=\varepsilon_{\mathrm{z}}\left(\cos 0^{\circ}\right)^{2}+\varepsilon_{\mathrm{x}}\left(\sin 0^{\circ}\right)^{2}+\gamma_{\mathrm{zx}} \sin 0^{\circ} \cos 0^{\circ} \\
\varepsilon_{3}=\varepsilon_{\mathrm{z}}\left(\cos \left(45^{\circ}\right)\right)^{2}+\varepsilon_{\mathrm{x}}\left(\sin \left(45^{\circ}\right)\right)^{2}+\gamma_{\mathrm{zx}} \sin \left(45^{\circ}\right) \cos \left(45^{\circ}\right)
\end{array}\right]
$$

where, $\varepsilon_{\mathrm{z}}$ and $\varepsilon_{\mathrm{x}}$ are normal strain in $\mathrm{z}$ and $\mathrm{x}$ direction respectively and $\gamma_{\mathrm{zx}}$ is the shear strain in $\mathrm{zx}$ direction.

Hence solving (C2) for $\varepsilon_{\mathrm{z}}, \varepsilon_{\mathrm{x}}$ and $\gamma_{\mathrm{zx}}$

$$
\begin{gathered}
\varepsilon_{\mathrm{z}}=\varepsilon_{2} \\
\varepsilon_{\mathrm{x}}=\varepsilon_{1}+\varepsilon_{3}-\varepsilon_{2} \\
\gamma_{\mathrm{zx}}=\varepsilon_{3}-\varepsilon_{1}
\end{gathered}
$$

Also, for normal stress $\sigma_{\mathrm{z}}$ and $\sigma_{\mathrm{x}}$,

$$
\begin{aligned}
\varepsilon_{\mathrm{z}} & =\frac{\sigma_{\mathrm{z}}}{\mathrm{E}}-\mathrm{v} \frac{\sigma_{\mathrm{x}}}{\mathrm{E}} \\
\varepsilon_{\mathrm{x}} & =\frac{\sigma_{\mathrm{x}}}{\mathrm{E}}-\mathrm{v} \frac{\sigma_{\mathrm{z}}}{\mathrm{E}} \\
\tau_{\mathrm{zx}} & =\gamma_{\mathrm{zx}} \mathrm{G}
\end{aligned}
$$


where, $v$ is the Poisson's ratio, $G$ is the modulus of rigidity and $E$ is the Young's Modulus of Elasticity.

Solving

$$
\begin{gathered}
\sigma_{x}=\frac{\left(\varepsilon_{x}+v \varepsilon_{z}\right) E}{\left(1-v^{2}\right)} \\
\sigma_{z}=\left(\varepsilon_{z}+v \frac{\sigma_{x}}{E}\right) E \\
\tau_{z x}=\gamma_{z x} G
\end{gathered}
$$

The equivalent von Mises stress is given by:

$$
\sigma_{\text {eq }}=\sqrt{\left(\left(\frac{\sigma_{z}-\sigma_{x}}{2}\right)^{2}+\left(\frac{\sigma_{z}-0}{2}\right)^{2}+\left(\frac{0-\sigma_{x}}{2}\right)^{2}+3 \tau_{\mathrm{zx}}{ }^{2}\right)}
$$




\section{Appendix D: Graphical Results from FEA}

1. P3G connection subjected to torsional bending loading of $80 \mathrm{Nm}$ and $4600 \mathrm{~N}$

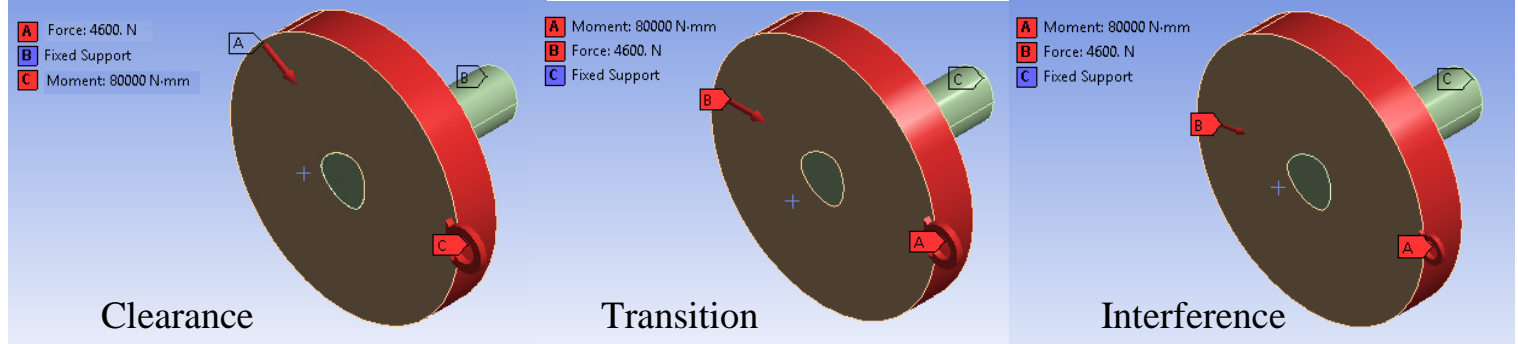

Figure D1: Torsional bending loading for P3G connection
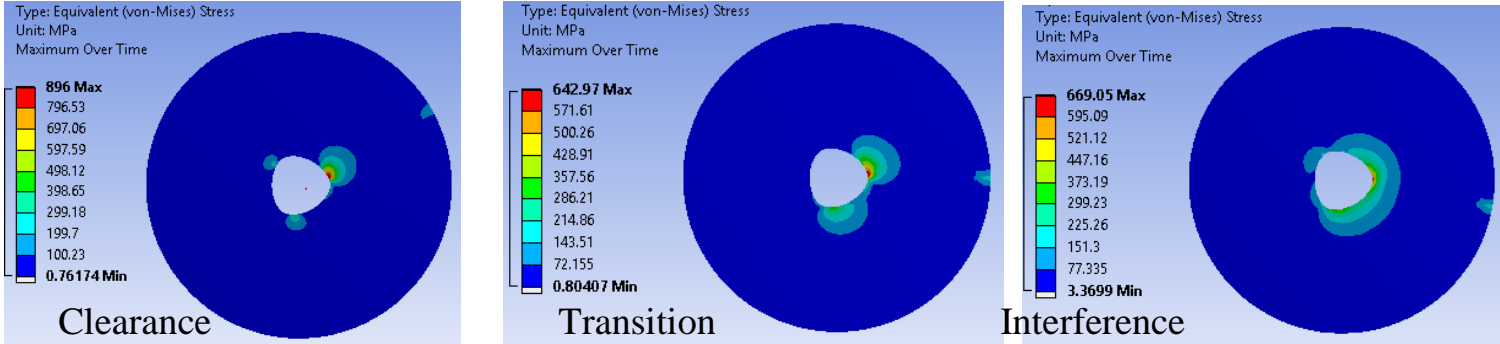

Figure D2: von Mises stress in P3G hub for torsional bending load

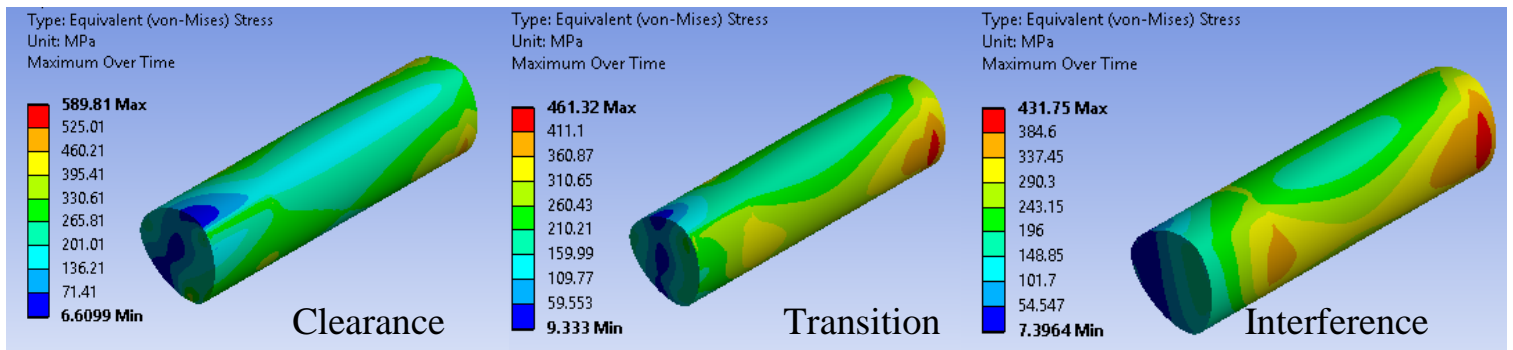

Figure D3: von Mises stress in P3G shaft for torsional bending load
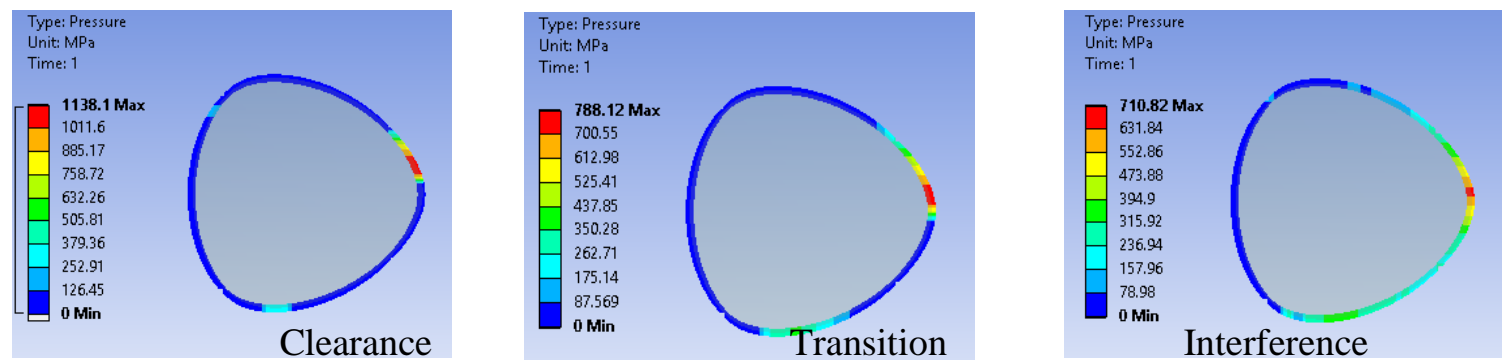

Figure D4: Contact stress in the P3G shaft along the edge for torsional bending load 

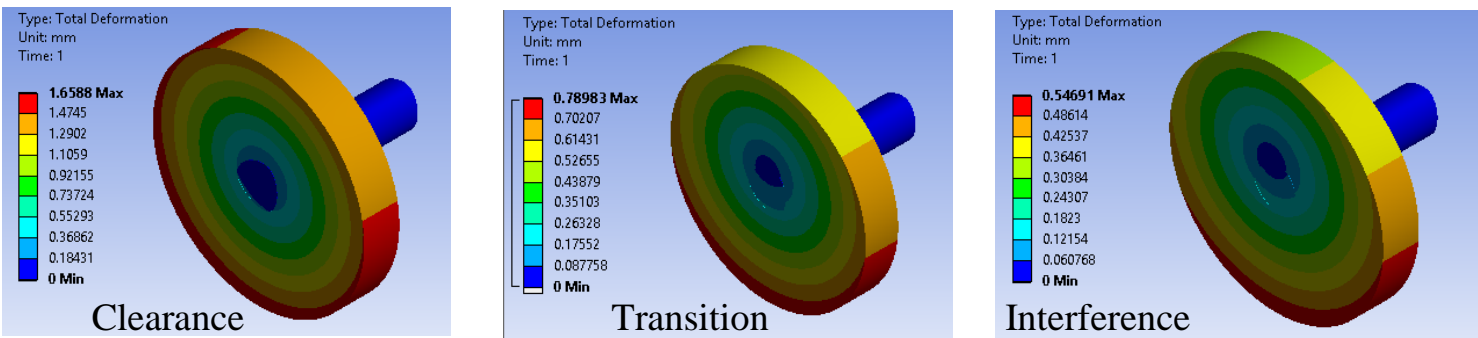

Figure D5: Total deformation for torsional bending load in P3G shaft

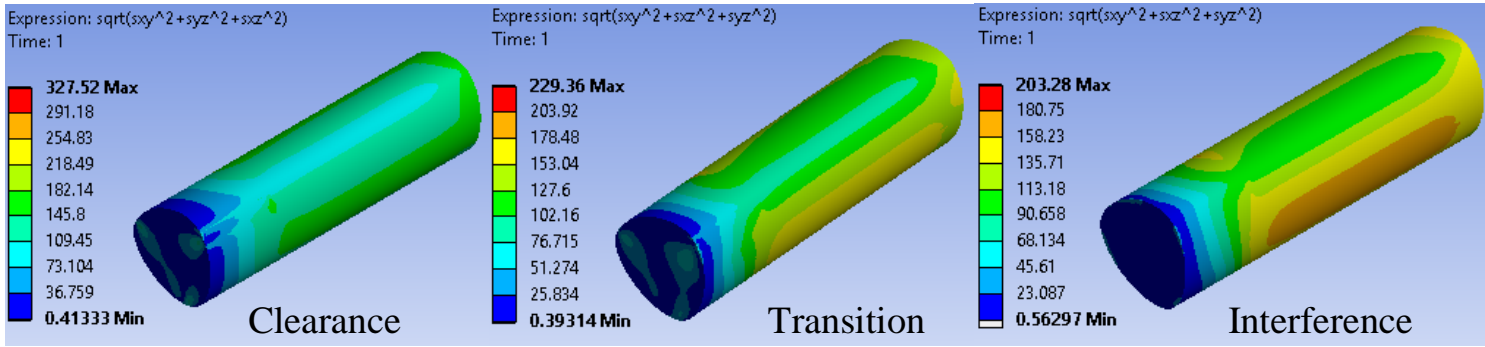

Figure D6: Torsional shear stress for torsional bending load in P3G shaft
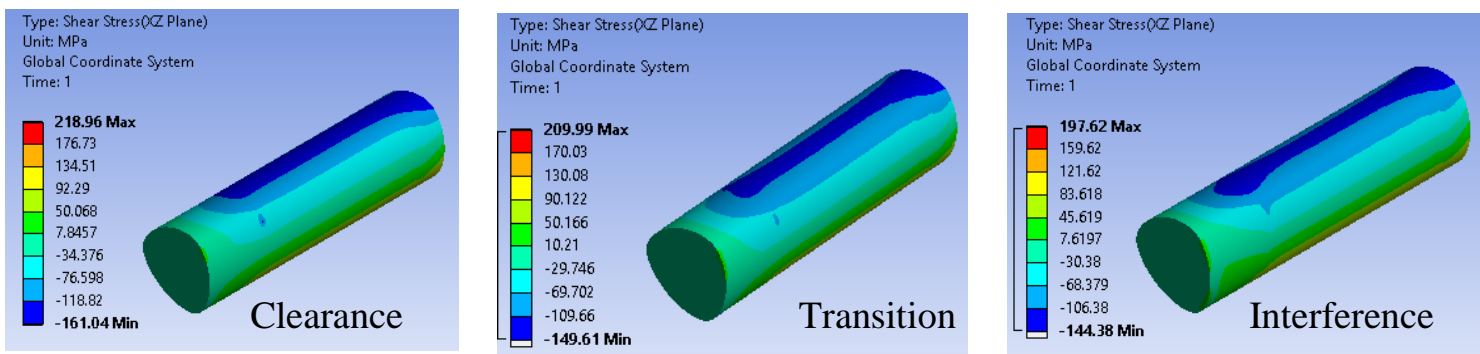

Figure D7: Shear stress in xz direction for torsional bending load in P3G shaft
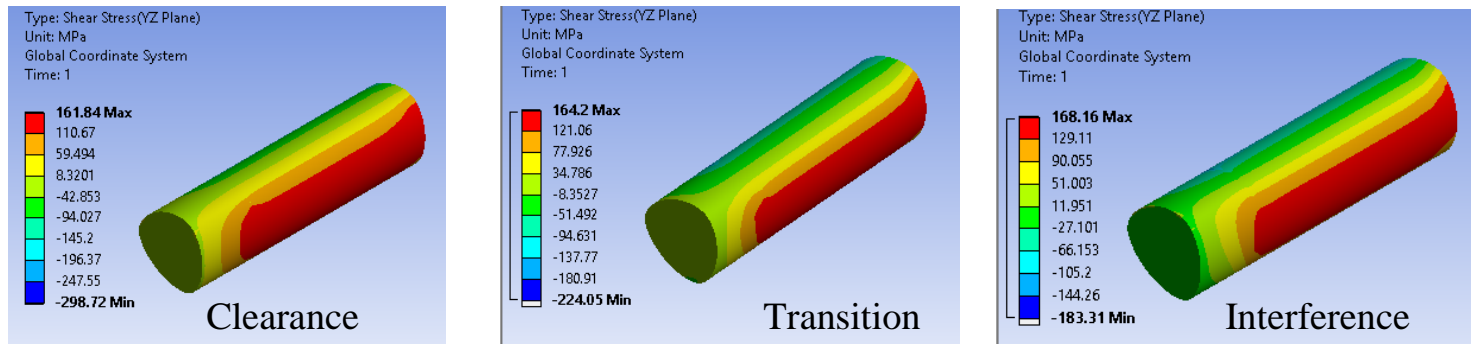

Figure D8: Shear stress in yz direction for torsional bending load in P3G shaft 
2. P4C connection subjected to torsional bending loading of $80 \mathrm{Nm}$ and $4600 \mathrm{~N}$

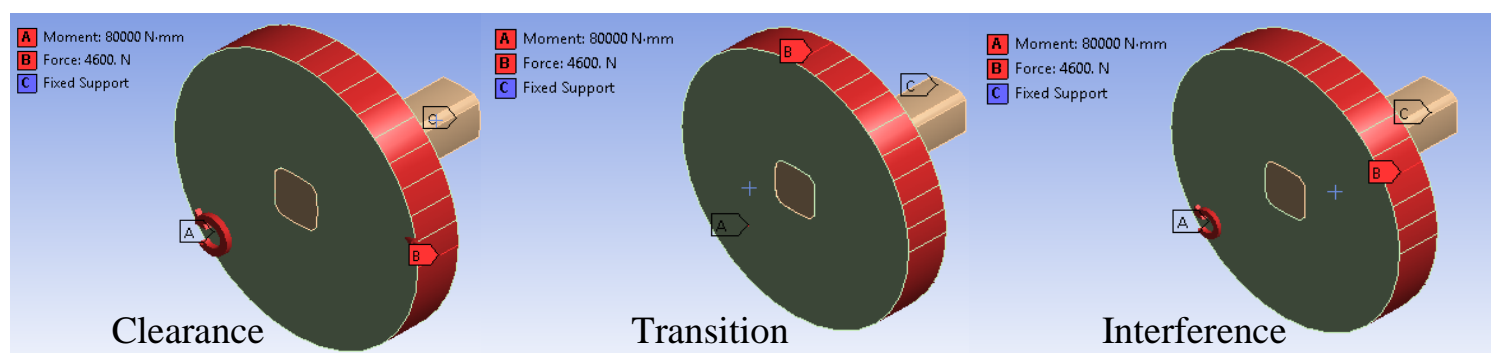

Figure D9: Torsional bending loading for P4C connection

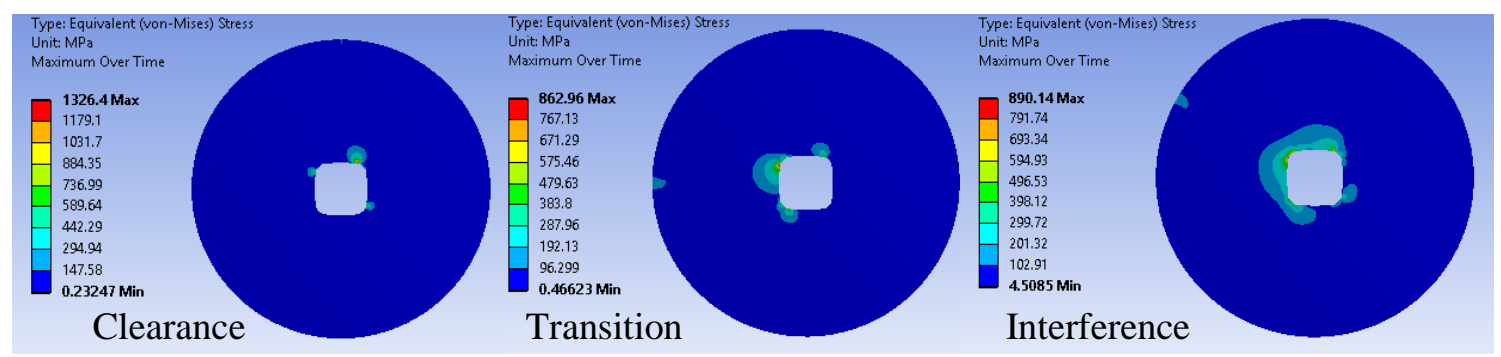

Figure D10: von Mises stress in P4C hub for torsional bending load

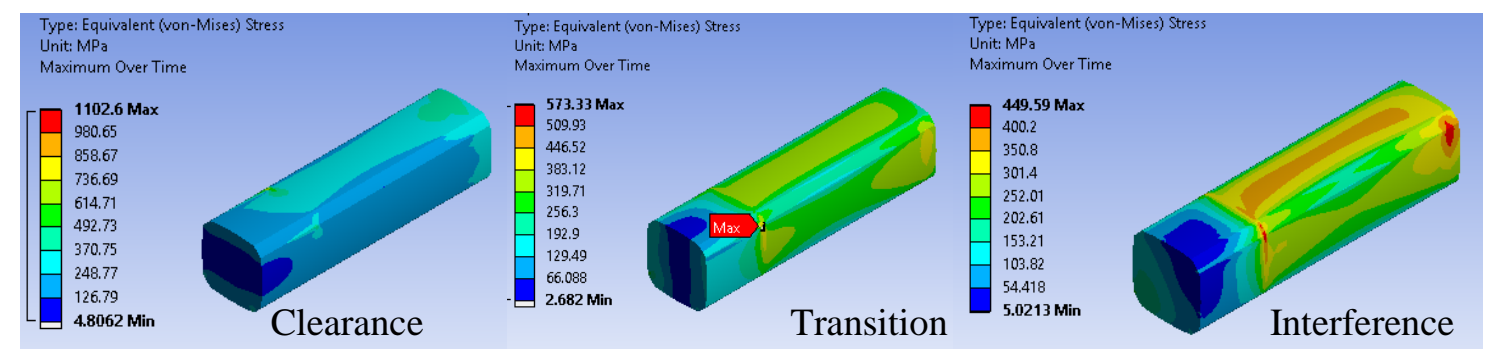

Figure D11: von Mises stress in P4C shaft for torsional bending load

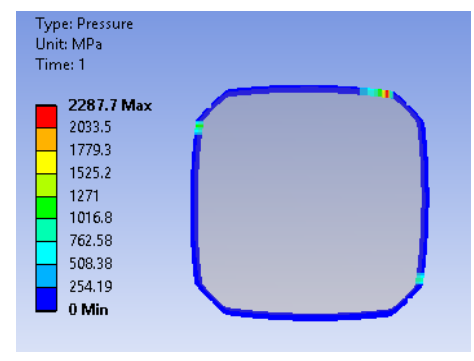

Clearance

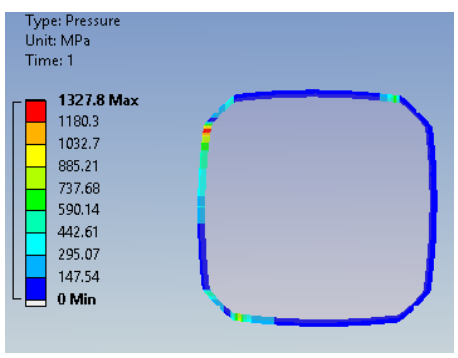

Transition

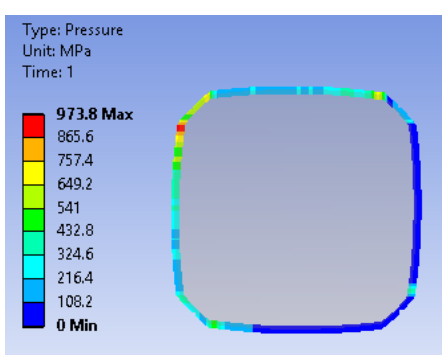

Interference

Figure D12: Contact stress in P4C shaft along the edge for torsional bending load 

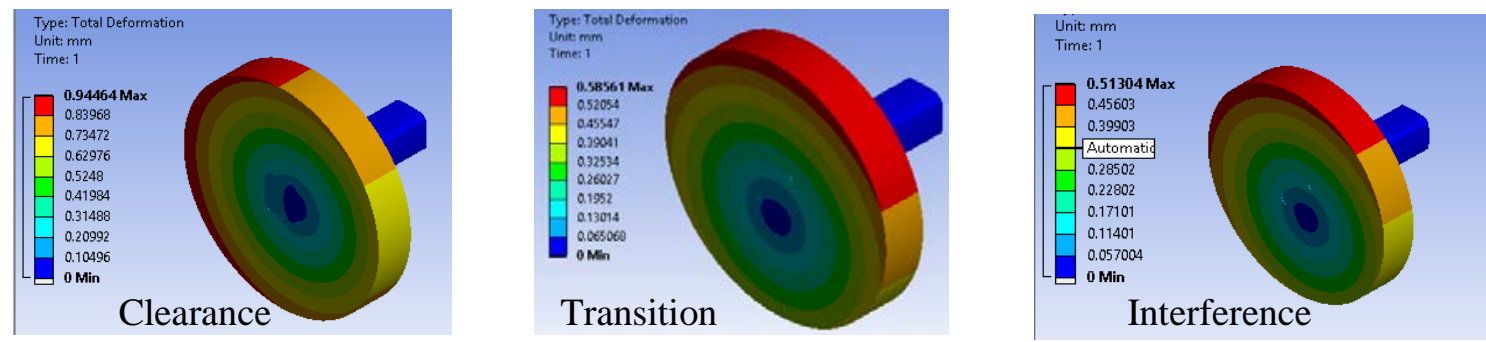

Figure D13: Total deformation for torsional bending load in P4C shaft
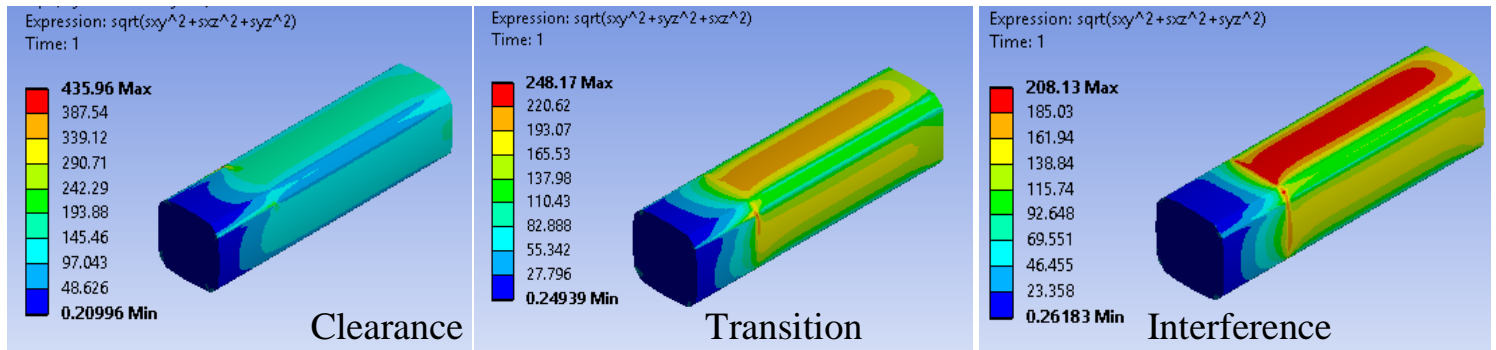

Figure D14: Torsional shear stress for torsional bending load in P4C shaft
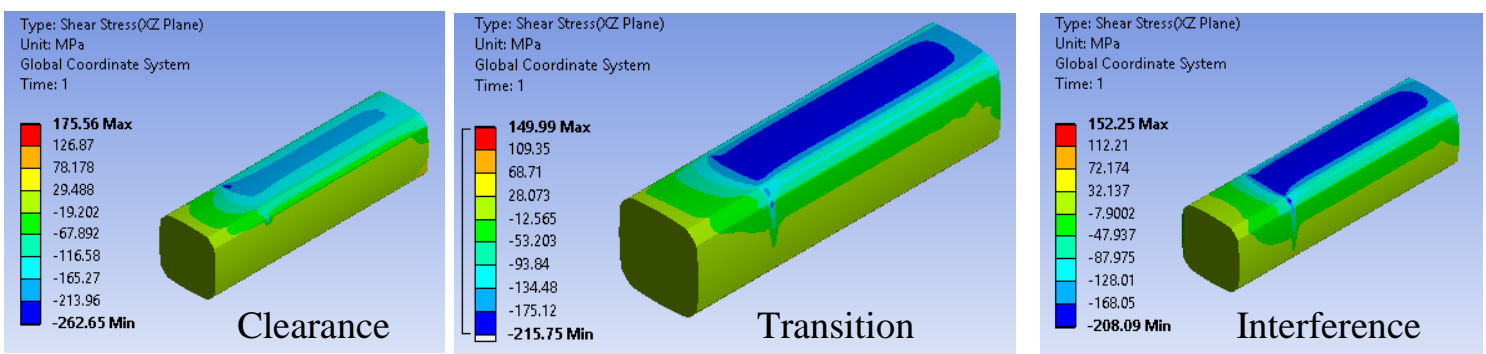

Figure D15: Shear stress in xz direction for torsional bending load in P4C shaft

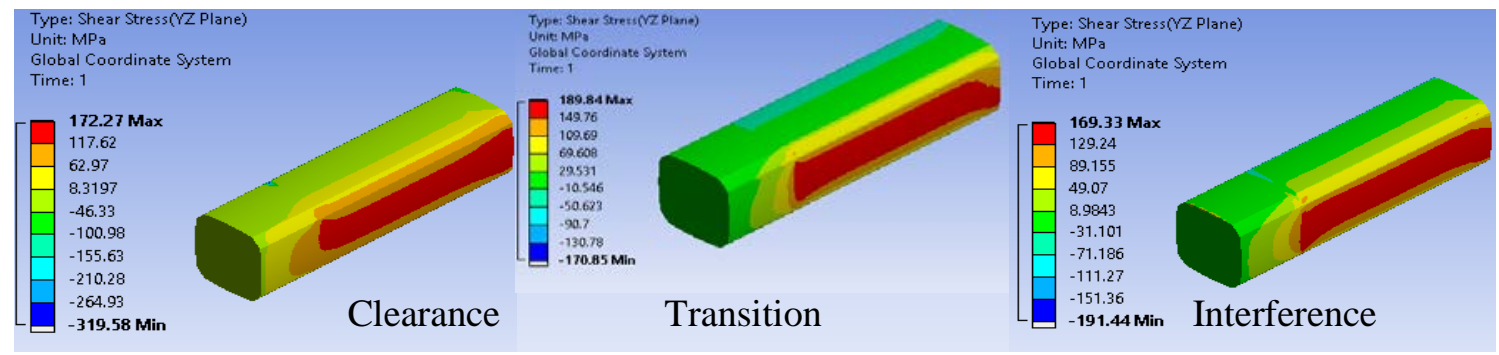

Figure D16: Shear stress in yz direction for torsional bending load in P4C shaft 
3. P3G connection subjected to torsional load of $80 \mathrm{Nm}$

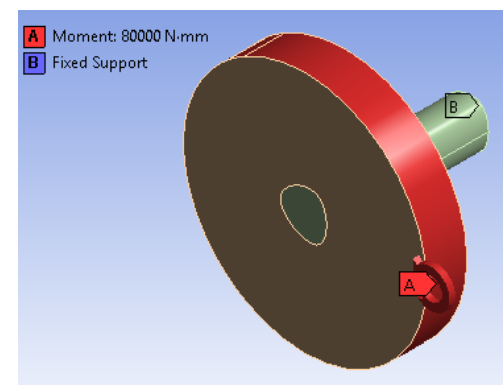

Figure D17: Torsional loading for P3G connection
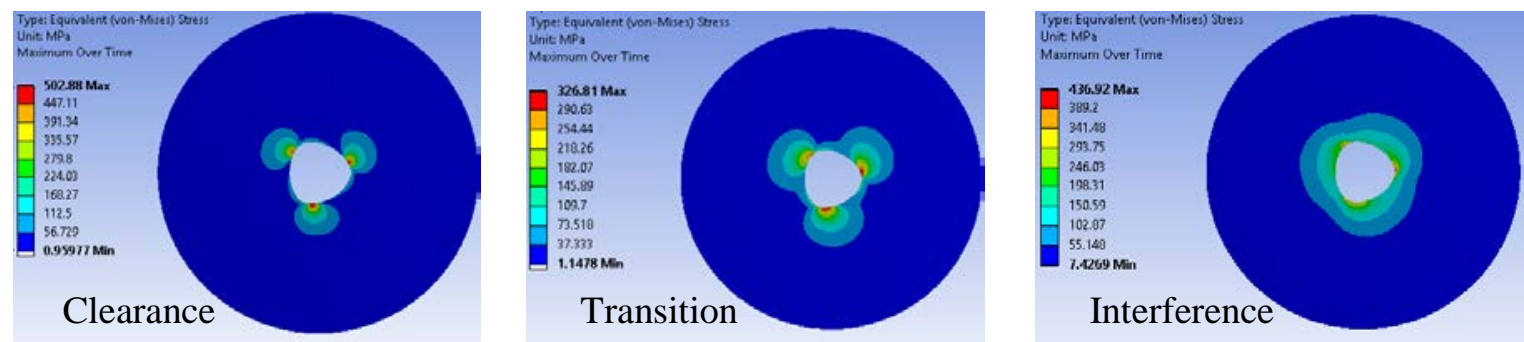

Figure D18: von Mises stress in P3G hub for torsional load
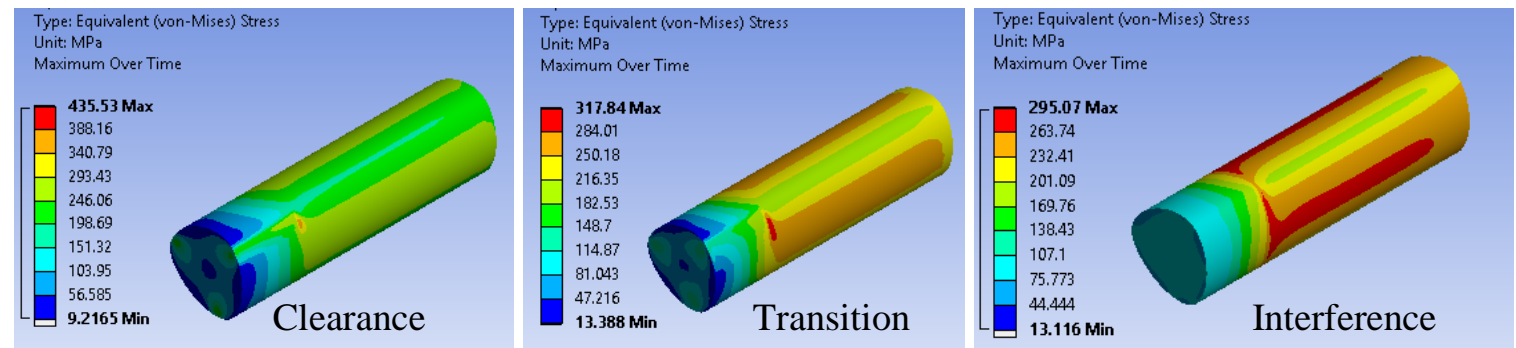

Figure D19: von Mises stress in P3G shaft for torsional load
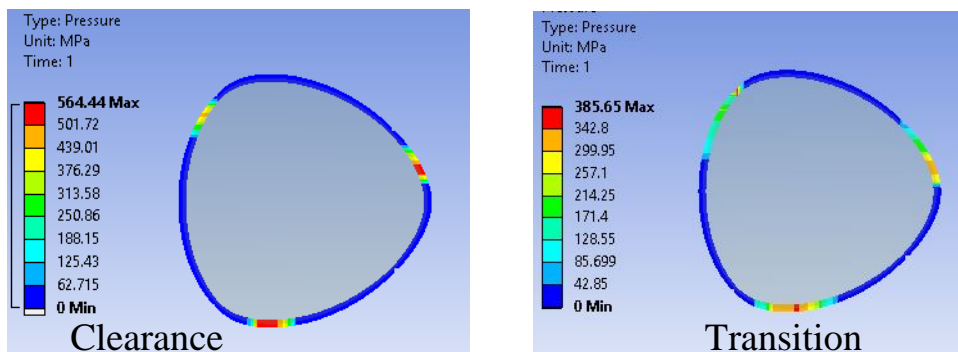

Transition

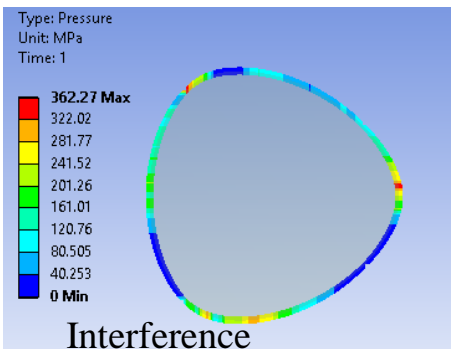

Figure D20: Contact stress in the P3G shaft along the edge for torsional load 

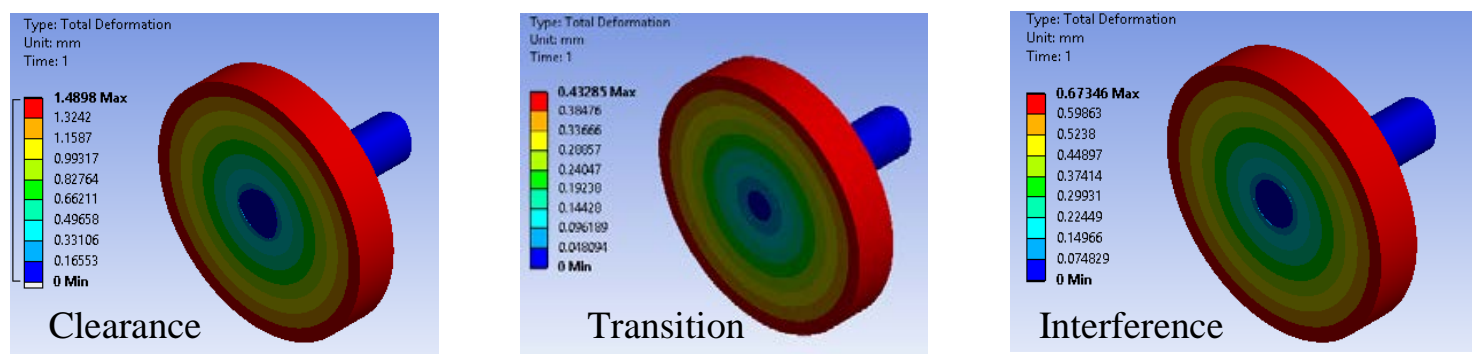

Figure D21: Total deformation for torsional load in P3G shaft

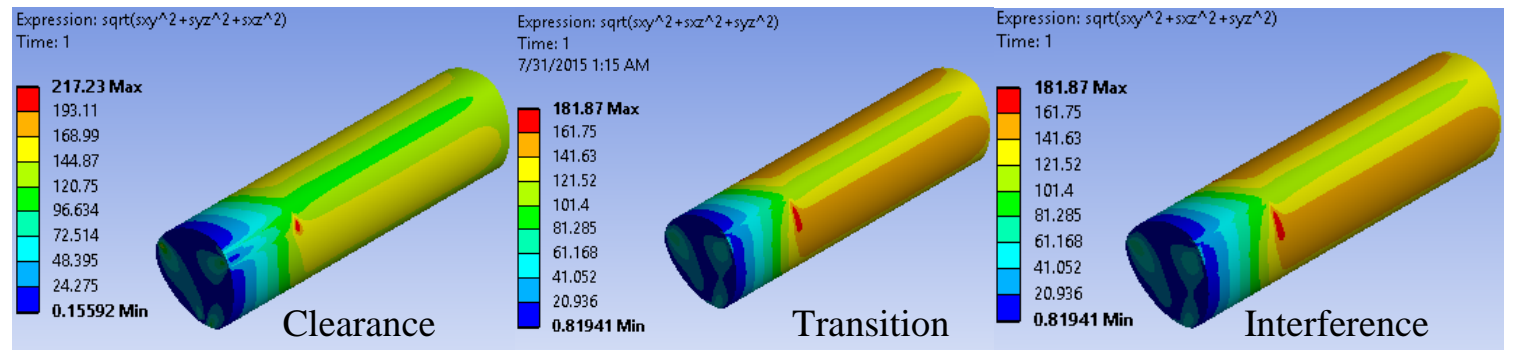

Figure D22: Torsional shear stress for torsional load in P3G shaft
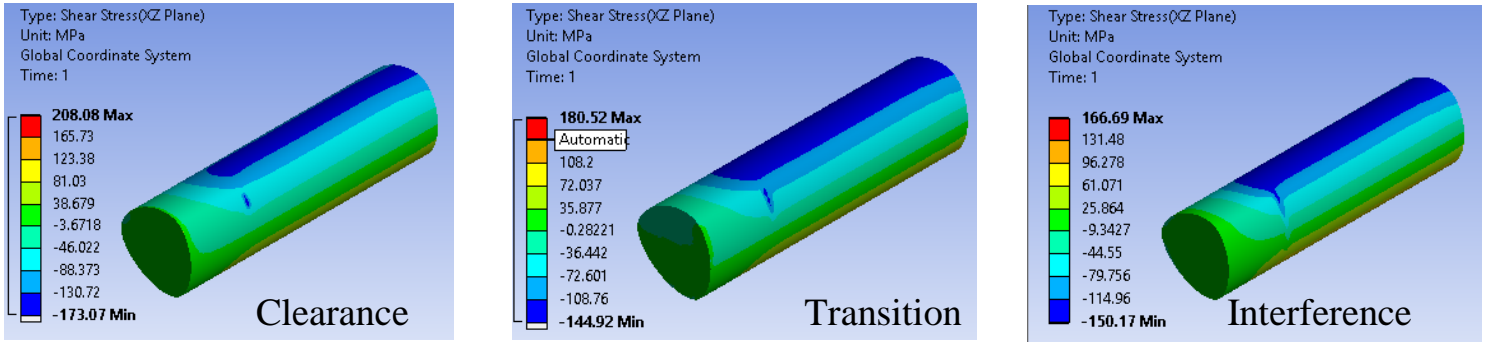

Figure D23: Shear stress in xz direction for torsional load in P3G shaft
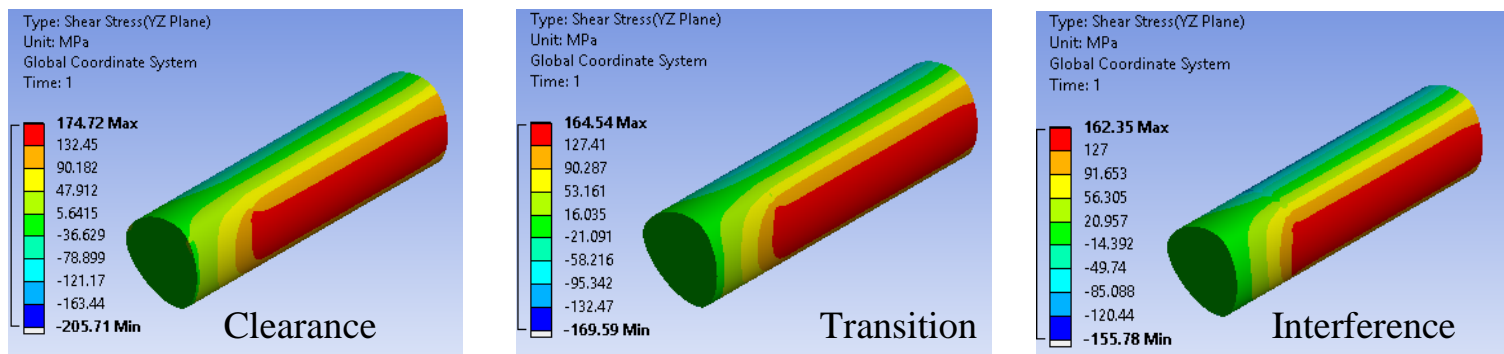

Figure D24: Shear stress in yz direction for torsional load in P3G shaft 
4. P4C connection subjected to torsional load of $80 \mathrm{Nm}$

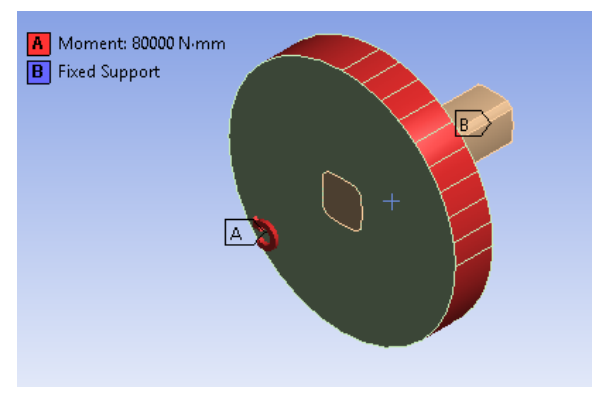

Figure D25: Torsional loading for P4C connection
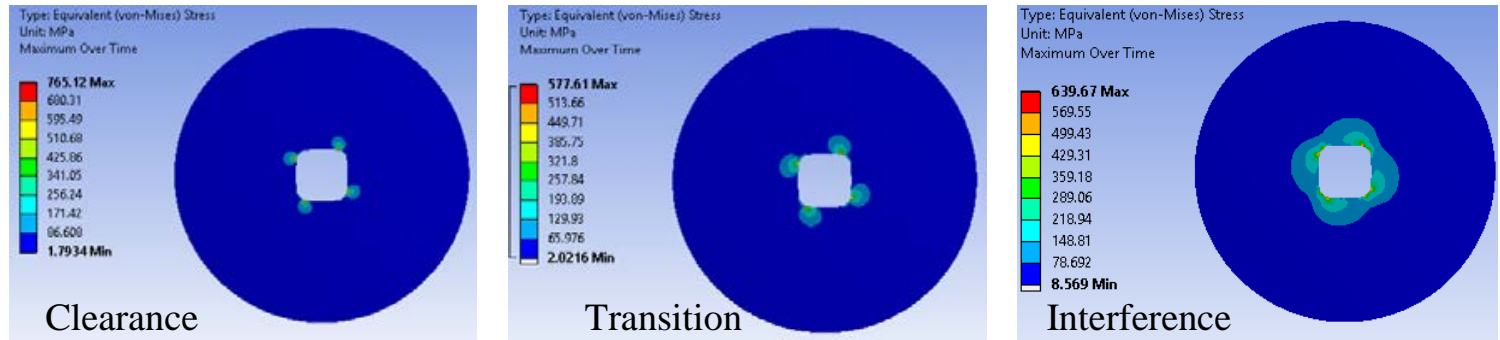

Figure D26: von Mises stress in P4C hub for torsional load
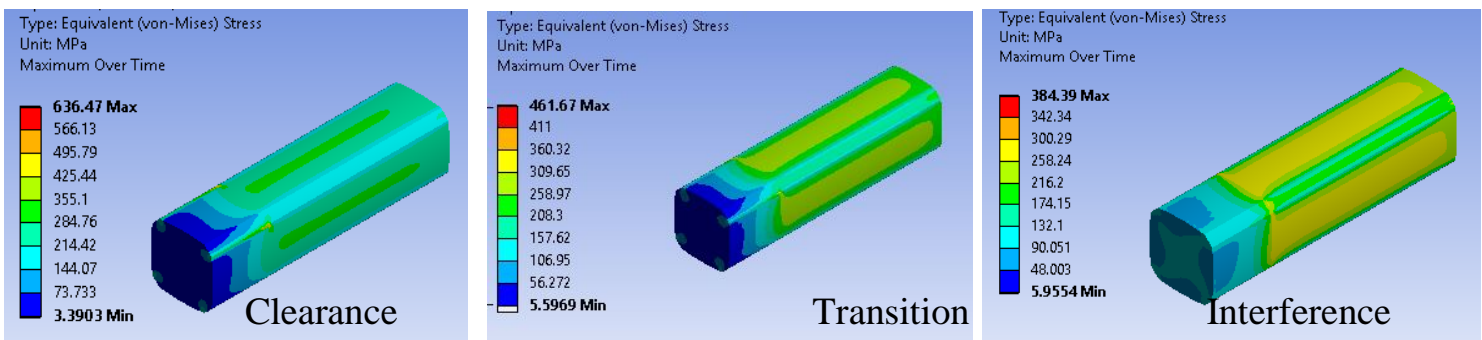

Figure D27: von Mises stress in P4C shaft for torsional load
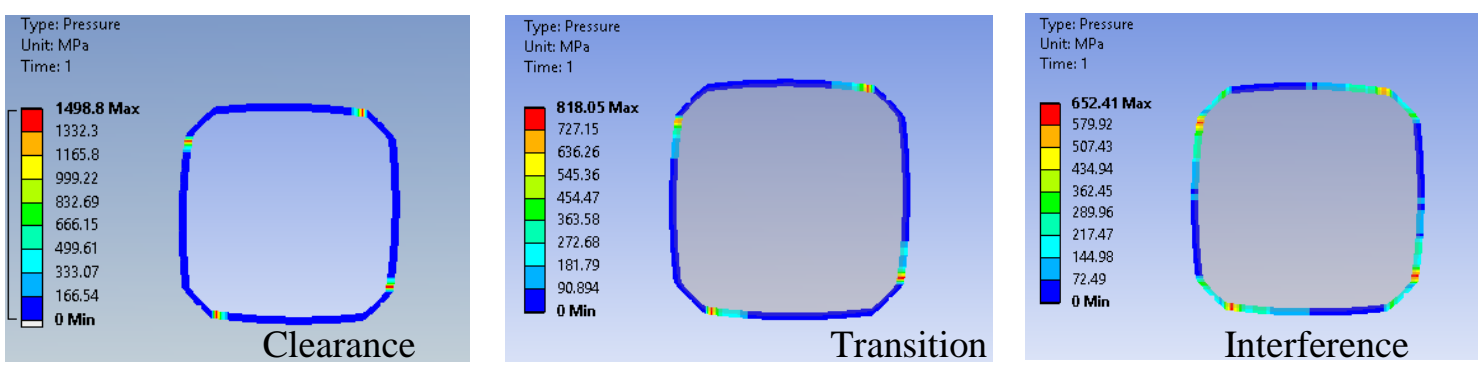

Figure D28: Contact stress in the P4C shaft along the edge for torsional load 

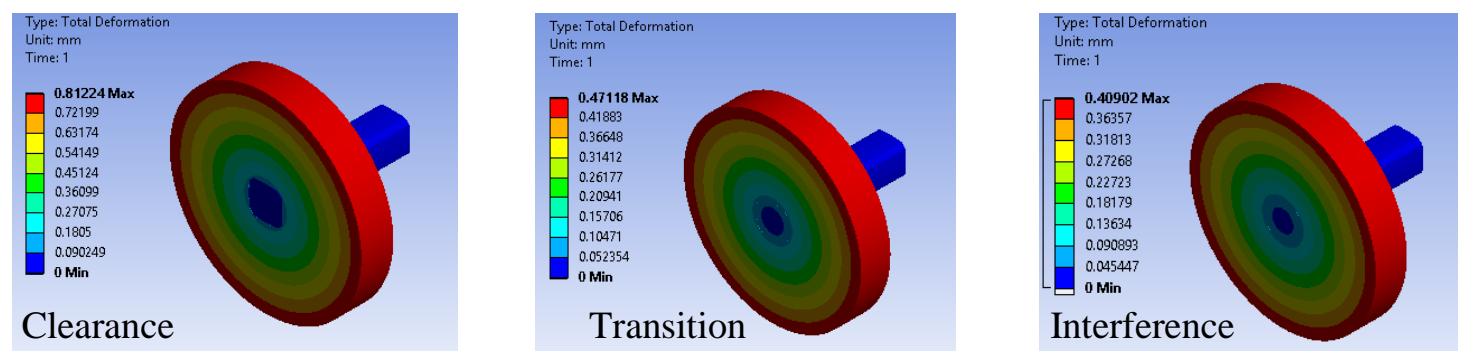

Figure D29: Total deformation for torsional load in P4C shaft

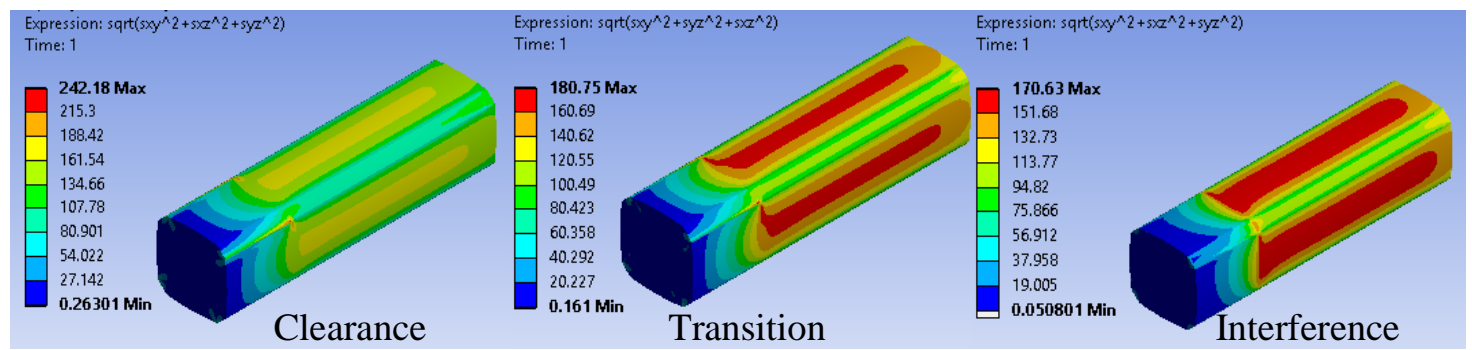

Figure D30: Torsional shear stress for torsional load in P4C shaft
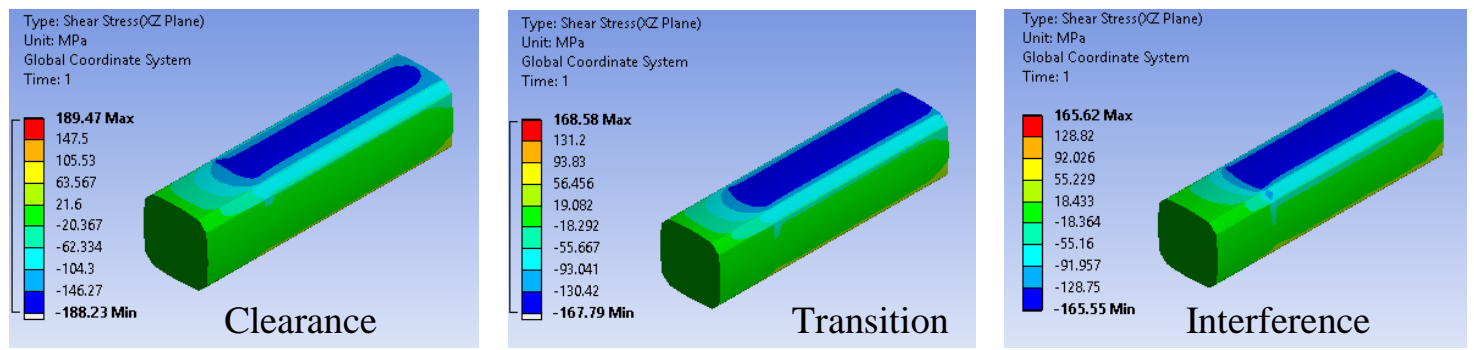

Figure D31: Shear stress in xz direction for torsional load in P4C shaft

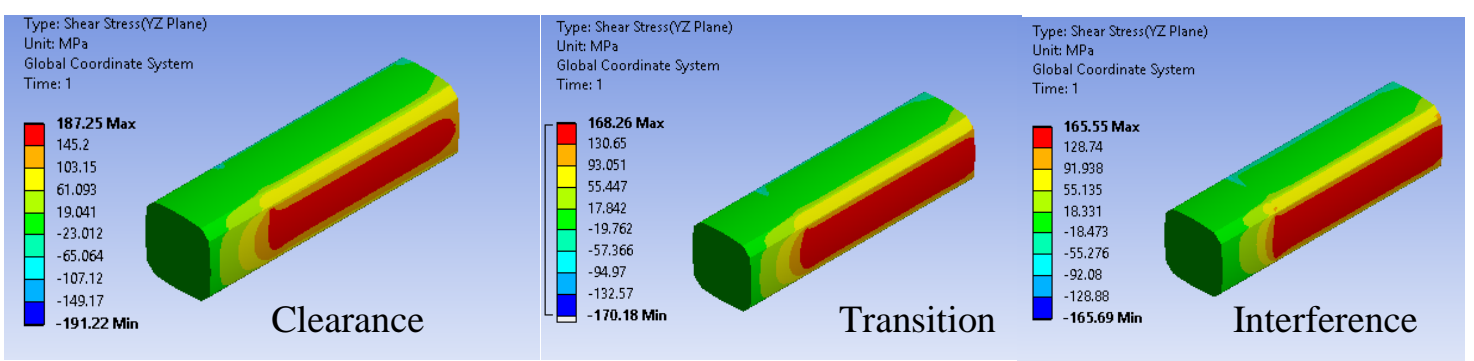

Figure D32: Shear stress in yz direction for torsional load in P4C shaft 
Table D1: Result of loading on P3G shaft for pure torsional loading of $80 \mathrm{Nm}$

\begin{tabular}{|c|c|c|c|c|}
\hline S. No. & Parameter & Clearance & Transition & Interference \\
\hline 1 & $\begin{array}{l}\text { Maximum von Mises Stress in hub } \\
(\mathrm{MPa})\end{array}$ & 502.88 & 326.81 & 436.92 \\
\hline 2 & $\begin{array}{l}\text { Maximum von Mises Stress in shaft } \\
\text { (MPa) }\end{array}$ & 435.53 & 317.84 & 295.07 \\
\hline 3 & $\begin{array}{l}\text { Maximum Contact stress in hub } \\
(\mathrm{MPa})\end{array}$ & 558.18 & 365.89 & 380.17 \\
\hline 4 & $\begin{array}{l}\text { Maximum Contact stress in shaft } \\
(\mathrm{MPa})\end{array}$ & 564.44 & 385.65 & 362.27 \\
\hline 5 & $\begin{array}{l}\text { Normal stress in axial direction at the } \\
\text { middle of shaft (MPa) }\end{array}$ & -4.78 & -6.97 & -22.60 \\
\hline 6 & $\begin{array}{l}\text { Shear stress in shaft in } \mathrm{ZX} \text { direction } \\
(\mathrm{MPa})\end{array}$ & 150.83 & 146.77 & 144.65 \\
\hline 7 & $\begin{array}{l}\text { Shear stress in shaft in } \mathrm{YZ} \text { direction } \\
(\mathrm{MPa})\end{array}$ & 158.13 & 157.40 & 154.82 \\
\hline 8 & $\begin{array}{l}\text { Maximum torsional shear stress in } \\
\text { shaft outside the contact region }(\mathrm{MPa})\end{array}$ & 158.3 & 157.53 & 154.91 \\
\hline
\end{tabular}

Table D2: Result of loading on P4C shaft for pure torsional loading of $80 \mathrm{Nm}$

\begin{tabular}{|c|c|c|c|c|}
\hline S. No. & Parameter & Clearance & Transition & Interference \\
\hline 1 & Maximum von Mises Stress in hub (MPa) & 765.12 & 577.61 & 639.67 \\
\hline 2 & Maximum von Mises Stress in shaft (MPa) & 636.47 & 461.67 & 384.39 \\
\hline 3 & Maximum Contact stress in hub (MPa) & 1498.80 & 947.40 & 710.63 \\
\hline 4 & Maximum Contact stress in shaft (MPa) & 1181.10 & 818.05 & 652.41 \\
\hline 5 & $\begin{array}{l}\text { Normal stress in axial direction at the middle } \\
\text { of shaft (MPa) }\end{array}$ & -2.10 & -3.33 & -22.25 \\
\hline 6 & Shear stress in shaft in ZX direction (MPa) & 167.88 & 166.70 & 165.62 \\
\hline 7 & Shear stress in shaft in YZ direction (MPa) & 167.89 & 166.74 & 165.55 \\
\hline 8 & $\begin{array}{l}\text { Maximum torsional shear stress in shaft } \\
\text { outside the contact region (MPa) }\end{array}$ & 167.92 & 166.80 & 165.63 \\
\hline
\end{tabular}


Table D3: Result of P3G and P4C loading for loading case 1 for clearance fit

\begin{tabular}{|l|l|r|r|r|}
\hline \multicolumn{1}{|c|}{$\begin{array}{c}\text { S. } \\
\text { No. }\end{array}$} & \multicolumn{1}{|c|}{ Parameter } & \multicolumn{1}{|c|}{ P3G } & \multicolumn{1}{c|}{ P4C } & \multicolumn{1}{c|}{$\begin{array}{c}\text { Percentage } \\
\text { Difference (\%) }\end{array}$} \\
\hline 1 & Maximum von Mises Stress in hub (MPa) & 135.58 & 187.03 & -31.90 \\
\hline 2 & Maximum von Mises Stress in shaft (MPa) & 111.29 & 134.84 & -19.14 \\
\hline 3 & $\begin{array}{l}\text { Average von Mises stress in hub portion connected to } \\
\text { shaft(MPa) }\end{array}$ & 22.60 & 21.73 & 3.93 \\
\hline 4 & $\begin{array}{l}\text { Average von Mises stress in shaft portion connected } \\
\text { to hub(MPa) }\end{array}$ & 12.288 & 7.30 & 50.93 \\
\hline 5 & Maximum Contact stress in hub (MPa) & 186.11 & 420.19 & -77.22 \\
\hline 6 & Maximum Contact stress in shaft (MPa) & 184.82 & 276.36 & -39.70 \\
\hline 7 & Average Contact stress in hub (MPa) & 78.20 & 175.67 & -76.79 \\
\hline 8 & Average Contact stress in shaft (MPa) & 75.53 & 47.75 & 45.07 \\
\hline 9 & $\begin{array}{l}\text { Normal stress in axial direction at the middle of shaft } \\
\text { (MPa) }\end{array}$ & 0.90 & 0.41 & 74.81 \\
\hline 10 & Displacement in axial direction of hub & -0.00048 & -0.00037 & 25.88 \\
\hline 11 & Shear stress in shaft in ZX direction (MPa) & 18.30 & 21.20 & -14.68 \\
\hline 12 & Shear stress in shaft in YZ direction (MPa) & 14.61 & 17.20 & -16.28 \\
\hline 13 & $\begin{array}{l}\text { Maximum torsional shear stress in shaft outside the } \\
\text { contact region }\end{array}$ & 20.12 & 20.90 & -3.80 \\
\hline
\end{tabular}

* The percentage difference is not in absolute value, meaning a negative value shows value in P4C connection was greater

Table D4: Result of P3G and P4C loading for loading case 2 for clearance fit

\begin{tabular}{|c|l|r|r|r|}
\hline $\begin{array}{c}\text { S. } \\
\text { No. }\end{array}$ & \multicolumn{1}{|c|}{ Parameter } & \multicolumn{1}{|c|}{ P3G } & \multicolumn{1}{|c|}{ P4C } & \multicolumn{1}{|c|}{$\begin{array}{l}\text { Percentage } \\
\text { Difference (\%) }\end{array}$} \\
\hline 1 & Maximum von Mises Stress in hub (MPa) & 896.00 & 1326.40 & -38.73 \\
\hline 2 & Maximum von Mises Stress in shaft (MPa) & 589.81 & 1102.60 & -60.60 \\
\hline 3 & $\begin{array}{l}\text { Average von Mises stress in hub portion connected to shaft } \\
\text { (MPa) }\end{array}$ & 121.97 & 73.84 & 49.16 \\
\hline 4 & $\begin{array}{l}\text { Average von Mises stress in shaft portion connected to hub } \\
\text { (MPa) }\end{array}$ & 221.03 & 218.02 & 1.37 \\
\hline 5 & Maximum Contact stress in hub (MPa) & 1160.30 & 2909.1 & -85.95 \\
\hline 6 & Maximum Contact stress in shaft (MPa) & 1138.10 & 2287.7 & -67.11 \\
\hline 7 & Average Contact stress in hub (MPa) & 321.92 & 562.83 & -54.46 \\
\hline 8 & Average Contact stress in shaft (MPa) & -13.8 & -8.00 & -43.59 \\
\hline 9 & Normal stress in axial direction in center of shaft (MPa) & -0.0056 & -0.0043 & 53.21 \\
\hline 10 & Maximum Displacement of hub in axial direction (mm) & 195.20 & 175.56 & 26.26 \\
\hline 11 & Shear stress in shaft in ZX direction (MPa) & 152.33 & 222.38 & 10.59 \\
\hline 12 & Shear stress in shaft in YZ direction (MPa) & 211.80 & 222.60 & -37.39 \\
\hline 13 & Maximum torsional shear stress in shaft (MPa) & -4.97 \\
\hline
\end{tabular}


Table D5: Result of P3G and P4C loading for loading case 1 for transition fit

\begin{tabular}{|c|c|c|c|c|}
\hline $\begin{array}{l}\text { S. } \\
\text { No. }\end{array}$ & Parameter & P3G & P4C & $\begin{array}{c}\text { Percentage } \\
\text { Difference (\%) }\end{array}$ \\
\hline 1 & Maximum von Mises Stress in hub (MPa) & 111.61 & 99.80 & 11.17 \\
\hline 2 & Maximum von Mises Stress in shaft (MPa) & 42.66 & 66.45 & -43.61 \\
\hline 3 & $\begin{array}{l}\text { Average von Mises stress in hub portion connected to } \\
\text { shaft(MPa) }\end{array}$ & 10.96 & 7.29 & 40.22 \\
\hline 4 & $\begin{array}{l}\text { Average von Mises stress in shaft portion connected } \\
\text { to hub(MPa) }\end{array}$ & 19.15 & 17.60 & 8.44 \\
\hline 5 & Maximum Contact stress in hub (MPa) & 167.12 & 161.04 & 3.71 \\
\hline 6 & Maximum Contact stress in shaft (MPa) & 156.49 & 147.00 & 6.25 \\
\hline 7 & Average Contact stress in hub (MPa) & 18.47 & 18.57 & -0.54 \\
\hline 8 & Average Contact stress in shaft (MPa) & 18.21 & 18.36 & -0.82 \\
\hline 9 & $\begin{array}{l}\text { Normal stress in axial direction at the middle of shaft } \\
\text { (MPa) }\end{array}$ & -28.72 & -20.89 & 31.57 \\
\hline 10 & Hub Max. Displacement in axial direction & -0.00028 & -0.00022 & 24.00 \\
\hline 1 & Shear stress in shaft in ZX direction (MPa) & 17.74 & 20.62 & -15.02 \\
\hline 12 & Shear stress in shaft in YZ direction (MPa) & 15.81 & 16.65 & -5.18 \\
\hline 13 & $\begin{array}{l}\text { Maximum torsional shear stress in shaft outside the } \\
\text { contact region }\end{array}$ & 19.00 & 20.60 & -8.08 \\
\hline
\end{tabular}

Table D6: Result of P3G and P4C loading for loading case 2 for transition fit

\begin{tabular}{|r|l|r|r|r|}
\hline \multicolumn{1}{|c|}{$\begin{array}{c}\text { S. } \\
\text { No. }\end{array}$} & \multicolumn{1}{|c|}{ Parameter } & \multicolumn{1}{|c|}{ P3G } & \multicolumn{1}{c|}{ P4C } & \multicolumn{1}{c|}{$\begin{array}{c}\text { Percentage } \\
\text { Difference (\%) }\end{array}$} \\
\hline 1 & Maximum von Mises Stress in hub (MPa) & 642.97 & 862.96 & -29.22 \\
\hline 2 & Maximum von Mises Stress in shaft (MPa) & 461.32 & 573.33 & -21.65 \\
\hline 3 & $\begin{array}{l}\text { Average von Mises stress in hub portion connected to shaft } \\
\text { (MPa) }\end{array}$ & 115.32 & 75.26 & 42.04 \\
\hline 4 & $\begin{array}{l}\text { Average von Mises stress in shaft portion connected to hub } \\
\text { (MPa) }\end{array}$ & 201.04 & 183.31 & 9.23 \\
\hline 5 & Maximum Contact stress in hub (MPa) & 800.06 & 1487.30 & -60.09 \\
\hline 6 & Maximum Contact stress in shaft (MPa) & 788.12 & 1327.80 & -51.01 \\
\hline 7 & Average Contact stress in hub (MPa) & 158.77 & 140.70 & 12.07 \\
\hline 8 & Average Contact stress in shaft (MPa) & -0.67 & -1.20 & 16.06 \\
\hline 9 & Normal stress in axial direction in center of shaft (MPa) & -0.0032 & -0.0025 & -56.68 \\
\hline 10 & Maximum Displacement of hub in axial direction (mm) & 187.00 & 215.75 & 24.56 \\
\hline 11 & Shear stress in shaft in ZX direction (MPa) & 164.20 & 178.00 & -14.28 \\
\hline 12 & Shear stress in shaft in YZ direction (MPa) & 199.30 & 215.75 & -8.07 \\
\hline 13 & Maximum torsional shear stress in shaft (MPa) & -7.93 \\
\hline
\end{tabular}


Table D7: Result of P3G and P4C loading for loading case 1 for interference fit

\begin{tabular}{|r|l|r|r|r|}
\hline \multicolumn{1}{|c|}{$\begin{array}{c}\text { S. } \\
\text { No. }\end{array}$} & \multicolumn{1}{|c|}{ Parameter } & P3G & \multicolumn{1}{|c|}{ P4C } & \multicolumn{1}{c|}{$\begin{array}{c}\text { Percentage } \\
\text { Difference (\%) }\end{array}$} \\
\hline 1 & Maximum von Mises Stress in hub (MPa) & 412.91 & 550.06 & -28.48 \\
\hline 2 & Maximum von Mises Stress in shaft (MPa) & 137.59 & 193.83 & -33.94 \\
\hline 3 & $\begin{array}{l}\text { Average von Mises stress in hub portion connected to } \\
\text { shaft(MPa) }\end{array}$ & 212.9 & 84.51 & 86.34 \\
\hline 4 & $\begin{array}{l}\text { Average von Mises stress in shaft portion connected to } \\
\text { hub(MPa) }\end{array}$ & 80.925 & 204.69 & -86.67 \\
\hline 5 & Maximum Contact stress in hub (MPa) & 318.09 & 347.1 & -8.72 \\
\hline 6 & Maximum Contact stress in shaft (MPa) & 279.88 & 326.34 & -15.33 \\
\hline 7 & Average Contact stress in hub (MPa) & 120.42 & 121.42 & -0.83 \\
\hline 8 & Average Contact stress in shaft (MPa) & -25.34 & -23.30 & -0.85 \\
\hline 9 & $\begin{array}{l}\text { Normal stress in axial direction at the middle of shaft } \\
\text { (MPa) }\end{array}$ & -0.0010 & -0.0011 & 8.39 \\
\hline 10 & Displacement in axial direction of hub & 18.26 & 20.60 & -9.52 \\
\hline 11 & Shear stress in shaft in ZX direction (MPa) & 16.30 & 19.10 & -12.04 \\
\hline 12 & Shear stress in shaft in YZ direction (MPa) & 19.12 & 20.80 & -15.82 \\
\hline 13 & $\begin{array}{l}\text { Maximum torsional shear stress in shaft outside the } \\
\text { contact region }\end{array}$ & & -8.42 \\
\hline
\end{tabular}

Table D8: Result of P3G and P4C loading for loading case 2 for interference fit

\begin{tabular}{|r|l|r|r|r|}
\hline \multicolumn{1}{|c|}{$\begin{array}{c}\text { S. } \\
\text { No. }\end{array}$} & \multicolumn{1}{|c|}{ Parameter } & P3G & \multicolumn{1}{|c|}{ P4C } & \multicolumn{1}{|c|}{$\begin{array}{c}\text { Percentage } \\
\text { Difference (\%) }\end{array}$} \\
\hline 1 & Maximum von Mises Stress in hub (MPa) & 669.05 & 890.14 & -28.36 \\
\hline 2 & Maximum von Mises Stress in shaft (MPa) & 431.75 & 449.59 & -4.05 \\
\hline 3 & $\begin{array}{l}\text { Average von Mises stress in hub portion connected to } \\
\text { shaft (MPa) }\end{array}$ & 207.29 & 202.62 & 2.28 \\
\hline & $\begin{array}{l}\text { Average von Mises stress in shaft portion connected to } \\
\text { hub (MPa) }\end{array}$ & 180.18 & 160.72 & 11.42 \\
\hline 5 & Maximum Contact stress in hub (MPa) & 726.35 & 1028.80 & -34.46 \\
\hline 6 & Maximum Contact stress in shaft (MPa) & 710.82 & 973.80 & -31.22 \\
\hline 7 & Average Contact stress in hub (MPa) & 121.25 & 125.80 & -3.68 \\
\hline 8 & Average Contact stress in shaft (MPa) & 121.40 & 125.75 & -3.52 \\
\hline 9 & Normal stress in axial direction in center of shaft (MPa) & -17.00 & -19.22 & -12.26 \\
\hline 10 & Maximum Displacement of hub in axial direction (mm) & -0.0016 & -0.0022 & -31.58 \\
\hline 11 & Shear stress in shaft in ZX direction (MPa) & 182.00 & 208.09 & -13.38 \\
\hline 12 & Shear stress in shaft in YZ direction (MPa) & 168.16 & 191.44 & -12.95 \\
\hline 13 & Maximum torsional shear stress in shaft (MPa) & 191.86 & 208.13 & -8.14 \\
\hline
\end{tabular}


Table D9: Result of FEA on P3G connection for loading case 1

\begin{tabular}{|r|l|r|r|r|}
\hline S. No. & \multicolumn{1}{|c|}{ Parameter } & Clearance & Transition & Interference \\
\hline 1 & Maximum von Mises Stress in hub (MPa) & 135.58 & 111.61 & 412.91 \\
\hline 2 & Maximum von Mises Stress in shaft (MPa) & 111.29 & 42.00 & 137.59 \\
\hline 3 & $\begin{array}{l}\text { Average von Mises stress in hub portion } \\
\text { connected to shaft(MPa) }\end{array}$ & 22.6 & 10.96 & 212.9 \\
\hline 4 & $\begin{array}{l}\text { Average von Mises stress in shaft portion } \\
\text { connected to hub(MPa) }\end{array}$ & 12.288 & 19.15 & 80.925 \\
\hline 5 & Maximum Contact stress in hub (MPa) & 186.11 & 167.12 & 318.09 \\
\hline 6 & Maximum Contact stress in shaft (MPa) & 184.82 & 156.49 & 279.88 \\
\hline 7 & Average Contact stress in hub (MPa) & 78.20 & 18.47 & 120.42 \\
\hline 8 & Average Contact stress in shaft (MPa) & 75.53 & 18.21 & 120.56 \\
\hline 9 & $\begin{array}{l}\text { Normal stress in axial direction at the middle } \\
\text { of shaft (MPa) }\end{array}$ & 0.90 & -28.72 & -25.34 \\
\hline 10 & Displacement in axial direction of hub & -0.00050 & -0.00028 & -0.0010 \\
\hline 11 & Shear stress in shaft in ZX direction (MPa) & 18.30 & 17.74 & 18.26 \\
\hline 12 & Shear stress in shaft in YZ direction (MPa) & 14.61 & 15.81 & 16.30 \\
\hline 13 & $\begin{array}{l}\text { Maximum torsional shear stress in shaft } \\
\text { outside the contact region }\end{array}$ & 20.12 & 19.00 & 19.12 \\
\hline
\end{tabular}

Table D10: Result of FEA on P3G connection for loading case 2

\begin{tabular}{|r|l|r|r|r|}
\hline S. No. & \multicolumn{1}{|c|}{ Parameter } & \multicolumn{1}{|c|}{ Clearance } & \multicolumn{1}{|c|}{ Transition } & Interference \\
\hline 1 & Maximum von Mises Stress in hub (MPa) & 896.00 & 642.97 & 669.05 \\
\hline 2 & Maximum von Mises Stress in shaft (MPa) & 589.81 & 461.32 & 431.75 \\
\hline 3 & $\begin{array}{l}\text { Average von Mises stress in hub portion } \\
\text { connected to shaft (MPa) }\end{array}$ & 121.97 & 115.32 & 207.29 \\
\hline 4 & $\begin{array}{l}\text { Average von Mises stress in shaft portion } \\
\text { connected to hub (MPa) }\end{array}$ & 221.03 & 201.04 & 180.18 \\
\hline 5 & Maximum Contact stress in hub (MPa) & 1160.30 & 800.06 & 726.35 \\
\hline 6 & Maximum Contact stress in shaft (MPa) & 1138.10 & 788.12 & 710.82 \\
\hline 7 & Average Contact stress in hub (MPa) & 321.92 & 158.77 & 121.25 \\
\hline 8 & Average Contact stress in shaft (MPa) & -13.80 & 159.20 & 121.40 \\
\hline 9 & $\begin{array}{l}\text { Normal stress in axial direction at the middle of } \\
\text { shaft (MPa) }\end{array}$ & -0.67 & -17.00 \\
\hline 10 & Displacement in axial direction of hub (mm) & 195.20 & 187.00 & 182.00 \\
\hline 11 & Shear stress in shaft in ZX direction (MPa) & 164.20 & 168.16 \\
\hline 12 & Shear stress in shaft in YZ direction (MPa) & 211.8 & 199.30 & 191.86 \\
\hline 13 & $\begin{array}{l}\text { Maximum torsional shear stress in shaft outside } \\
\text { the contact region (MPa) }\end{array}$ & & -0.0032 & -0.0016 \\
\hline
\end{tabular}


Table D11: Result of FEA on P4C connection for loading case 1

\begin{tabular}{|r|l|r|r|r|}
\hline S. No. & Parameter & Clearance & Transition & Interference \\
\hline 1 & Maximum von Mises Stress in hub (MPa) & 187.03 & 99.80 & 550.06 \\
\hline 2 & Maximum von Mises Stress in shaft (MPa) & 134.84 & 66.45 & 193.83 \\
\hline 3 & $\begin{array}{l}\text { Average von Mises stress in hub portion } \\
\text { connected to shaft(MPa) }\end{array}$ & 21.73 & 7.29 & 84.51 \\
\hline 4 & $\begin{array}{l}\text { Average von Mises stress in shaft portion } \\
\text { connected to hub(MPa) }\end{array}$ & 7.30 & 17.60 & 204.69 \\
\hline 5 & Maximum Contact stress in hub (MPa) & 420.19 & 161.04 & 347.1 \\
\hline 6 & Maximum Contact stress in shaft (MPa) & 175.67 & 18.57 & 121.42 \\
\hline 7 & Average Contact stress in hub (MPa) & -0.41 & -0.68 & 121.59 \\
\hline 8 & Average Contact stress in shaft (MPa) & -0.00037 & -0.00022 & -0.00110 \\
\hline 9 & $\begin{array}{l}\text { Normal stress in axial direction at the middle of } \\
\text { shaft (MPa) }\end{array}$ & -21.20 & -20.62 & -20.60 \\
\hline 10 & Displacement in axial direction of hub & 17.20 & 16.65 & -19.10 \\
\hline 11 & Shear stress in shaft in ZX direction (MPa) & 20.90 & 20.60 & 20.80 \\
\hline 12 & Shear stress in shaft in YZ direction (MPa) & & \\
\hline 13 & $\begin{array}{l}\text { Maximum torsional shear stress in shaft outside } \\
\text { the contact region }\end{array}$ & & & \\
\hline
\end{tabular}

Table D12: Result of FEA on P4C connection for loading case 2

\begin{tabular}{|r|l|r|r|r|}
\hline \multicolumn{1}{|l|}{ S. No. } & \multicolumn{1}{|c|}{ Parameter } & \multicolumn{1}{c|}{ Transition } & \multicolumn{1}{c|}{ Clearance } & \multicolumn{1}{c|}{ Interference } \\
\hline 1 & Maximum von Mises Stress in hub (MPa) & 862.96 & 1326.4 & 890.14 \\
\hline 2 & Maximum von Mises Stress in shaft (MPa) & 573.33 & 1102.6 & 449.59 \\
\hline 3 & $\begin{array}{l}\text { Average von Mises stress in hub portion } \\
\text { connected to shaft(MPa) }\end{array}$ & 75.26 & 73.84 & 202.62 \\
\hline 4 & $\begin{array}{l}\text { Average von Mises stress in shaft portion } \\
\text { lonnected to hub(MPa) }\end{array}$ & 183.31 & 218.02 & 160.72 \\
\hline 5 & Maximum Contact stress in hub (MPa) & 1487.30 & 2909.10 & 1028.80 \\
\hline 6 & Maximum Contact stress in shaft (MPa) & 140.70 & 562.83 & 973.80 \\
\hline 7 & Average Contact stress in hub (MPa) & -1.20 & -8.00 & 125.80 \\
\hline 8 & Average Contact stress in shaft (MPa) & -0.0025 & -0.0043 & -19.22 \\
\hline 9 & $\begin{array}{l}\text { Normal stress in axial direction at the middle of } \\
\text { shaft (MPa) }\end{array}$ & -215.75 & 175.56 & -0.0022 \\
\hline 10 & Displacement in axial direction of hub & 178.00 & -222.38 & -191.44 \\
\hline 11 & Shear stress in shaft in ZX direction (MPa) & 215.75 & 222.60 & 208.13 \\
\hline 12 & Shear stress in shaft in YZ direction (MPa) & $\begin{array}{l}\text { Maximum torsional shear stress in shaft outside } \\
\text { the contact region }\end{array}$ & & \\
\hline
\end{tabular}




\section{Appendix E: Permission to publish Figure 9}

Dear Mr. Bhatta,

It is our pleasure to grant you permission to publish and post the ASME Figures 5,6 from

“Analysis of Contact Stress and Deformation in a Trilobe Polygonal Connection,” by T.

Czyzewski and M. T. Odman, J. Eng. Ind 110(3), 1988, as cited in your letter for inclusion in a

MS-Engineering thesis entitled ScholarWorks@GVSU to be published by Grand Valley State

University.

Permission is granted for the specific use as stated herein and does not permit further use of the materials without proper authorization. Proper attribution must be made to the author(s) of the materials. As is customary, we request that you ensure proper acknowledgment of the exact sources of this material, the authors, and ASME as original publisher. Acknowledgment must be retained on all pages printed and distributed.

Many thanks for your interest in ASME publications.

Sincerely,

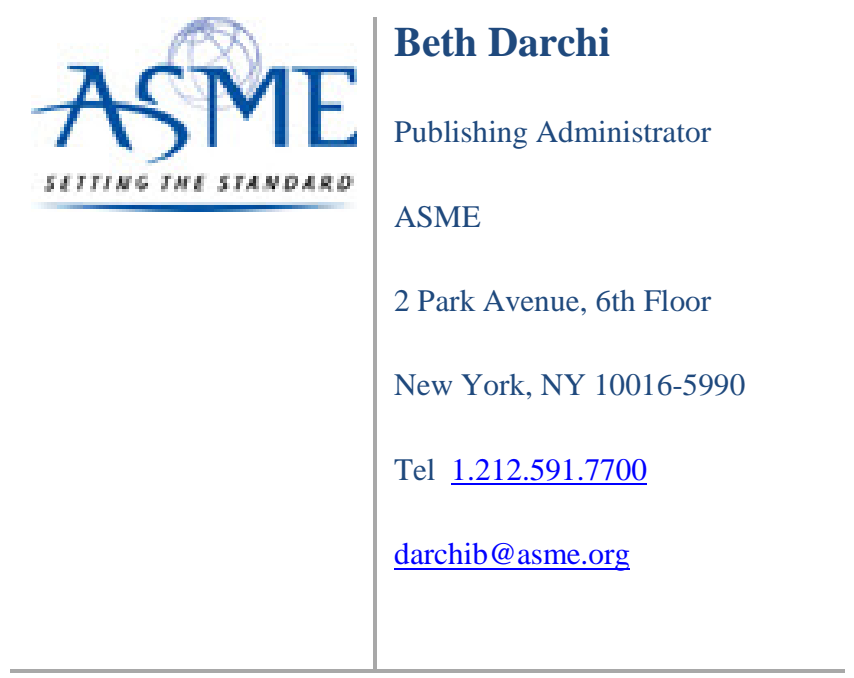




\section{References}

1. ANSYS INC, (2010). Lecture 3: Introduction to contact ANSYS Mechanical Structural Nonlinearities, , Retrieved from web on Aug 9, 2015 from http:// inside.mines.edu/ apetrell/ENME442/Labs/1301_ENME442_lab6_lecture.pdf

2. ANSYS INC., (2014). Workbench Mechanical Contact Best Practices, Retrieved from web on Aug 9, 2015 from http://www.ansys.com/staticassets/ANSYS/staticassets/ resourcelibrary/presentation/2014-sd-mechanical-contact-best-practices.pdf

3. B. Z. Lü, K. Liu, (2011). Research on Stress and Strain in Isometric Polygonal Profile Connection Based on FEM, Key Engineering Materials, Vols 460-461, 369-373

4. Bhashyam, Grama, R., (2002). ANSYS Mechanical- A Powerful Nonlinear Simulation Tool [PDF] , Corporate Fellow, Mechanics \& Simulation Support Group, ANSYS Inc., 275 Technology Drive, Canonsburg, PA 15317, Retrieved from web on on Aug 9, 2015 from http://www.umaine.edu/mecheng/files/2013/ 01/ANSYS_nonlinear.pdf

5. Citarella, R., \& Gerbino, S., (2001), BE analysis of shaft-hub couplings with polygonal profiles, Journal of Materials Processing Tech, 109(1), 30-37.

6. Czyzewski, T. \& Odman, M.T, (1988). Analysis of Contact Stress and Deformation in a Trilobe Polygonal Connection. Journal of Engineering for Industry, 110, 212-217.

7. DU, Yanxia, (2012). FEA on isometric polygonal profile connection of mining machine based on ANSYS, Mining \& Processing Equipment, China, Vol 40.

8. Filemon, E., (1959) Production and Analysis of Polygon Profiles, Periodica Polytechnica M III/1, Budapest 
9. Grossman, C., (2007). Fretting fatigue of shape optimised polygon-shaft-hub connections. (Doctoral Dissertation), Technical University Berlin.

10. Higgins, J., (2012) Obtaining and Optimizing Structural Analysis Convergence, Retrieved from web on Aug 9, 2015 from http://www.ansys.com/staticassets/ ANSYS/Conference/Confidence/Boston/Downloads/obtaining-and-optimizingconvergence.pdf

11. Huang, B., Li, J., Sun, Q. J., \& Wang, L. Q., (2010). Performance analysis of trilobe equidistant polygon connection [J]. Journal of Zhejiang University (Engineering Science), 3, 039.

12. Kahn-Jetter, Z. L., Hundertmark, E., \& Wright, S., (2000). Comparison of torque transmitting shaft connectivity using a trilobe polygon connection and an involute spline. Journal of Mechanical Design, 122(1), 130.

13. Lee, Huei-Huang, (2014). Finite Element Simulations with Ansys Workbench 15 Theory Applications Case studies, Mission, Kansas: SDC Publications

14. Maximov, J. T., (2005). A new method of manufacture of hypocycloidal polygon shaft joints. Journal of Materials Processing Tech, 166(1), 144-149.

15. Mohan, Pradeep, (2015). Lecture 2: Finite Element Modeling and Mesh Quality Checks [PDF] , The George Washington University, Retrieved from web on Aug 9, 2015 from http://crash.ncac.gwu.edu/pradeep/CE264/lecture_2/ lecture_2.pdf

16. Rossino, L. S., Castro, D. B. V. d., Moreto, J. A., Ruchert, C. O. F. T., Spinelli, D., \& Tarpani, J. R., (2014). Surface contact fatigue failure of a case hardened pinion shaft. Materials Research, 17(3) 
17. Slivker, V., (2007). Mechanics of structural elements: Theory and applications. Berlin, Heidelberg: Springer Berlin Heidelberg.

18. Standard DIN 32711, (2009-03) Polygonprofile P3G. Ausg. Berlin: Beuth Verlag

19. Standard DIN 32712, (2009-03) Polygonprofile P4C. Ausg. Berlin: Beuth Verlag

20. Steven E. Benzley, Ernest Perry, Karl Merkley, Brett Clark, (1995). A Comparison of All Hexagonal and All Tetrahedral Finite Element Meshes for Elastic and Elasto-plastic Analysis, Sandia National Laboratories, Albuquerque, NM, 179-191

21. Taylor, B., (1987). The design of a lathe attachment for grinding non-circular cross-section shafts suitable for torque transmission (Doctoral dissertation). Retrieved from Newcastle University eTheses from URL http://hdl.handle.net/10443/769

22. Winterfeld, J., (2001). Einflüsse der Reibdauerbeanspruchung auf die Tragfähigkeit von P4C-Welle-Nabe-Verbindungen. (Doctoral Dissertation), Technical University Berlin

23. Zehsaz, M., \& Shahriary, P., (2013). The effects of friction coefficient and interference on the fretting fatigue strength of railway axle assembly. U.P.B. Scientific Bulletin, Vol 75, Issue 4 